\title{
Does size matter?
}

Citation for published version (APA):

van den Berg, F. C. G. (2021). Does size matter? Behavioral and neural insights into the representation of small and large number symbols. [Doctoral Thesis, Maastricht University]. Maastricht University. https://doi.org/10.26481/dis.20211214fb

\section{Document status and date:}

Published: 01/01/2021

DOI:

$10.26481 /$ dis.20211214fb

Document Version:

Publisher's PDF, also known as Version of record

\section{Please check the document version of this publication:}

- A submitted manuscript is the version of the article upon submission and before peer-review. There can be important differences between the submitted version and the official published version of record.

People interested in the research are advised to contact the author for the final version of the publication, or visit the DOI to the publisher's website.

- The final author version and the galley proof are versions of the publication after peer review.

- The final published version features the final layout of the paper including the volume, issue and page numbers.

Link to publication

\footnotetext{
General rights Owners
rights.

- You may freely distribute the URL identifying the publication in the public portal. please follow below link for the End User Agreement:

www.umlib.nl/taverne-license

Take down policy

If you believe that this document breaches copyright please contact us at:

repository@maastrichtuniversity.nl

providing details and we will investigate your claim.
}

Copyright and moral rights for the publications made accessible in the public portal are retained by the authors and/or other copyright owners and it is a condition of accessing publications that users recognise and abide by the legal requirements associated with these

- Users may download and print one copy of any publication from the public portal for the purpose of private study or research.

- You may not further distribute the material or use it for any profit-making activity or commercial gain

If the publication is distributed under the terms of Article $25 \mathrm{fa}$ of the Dutch Copyright Act, indicated by the "Taverne" license above, 


\section{DOES SIZE MATTER?}

BEHAVIORAL AND NEURAL INSIGHTS INTO

THE REPRESENTATION OF SMALL AND LARGE NUMBER SYMBOLS

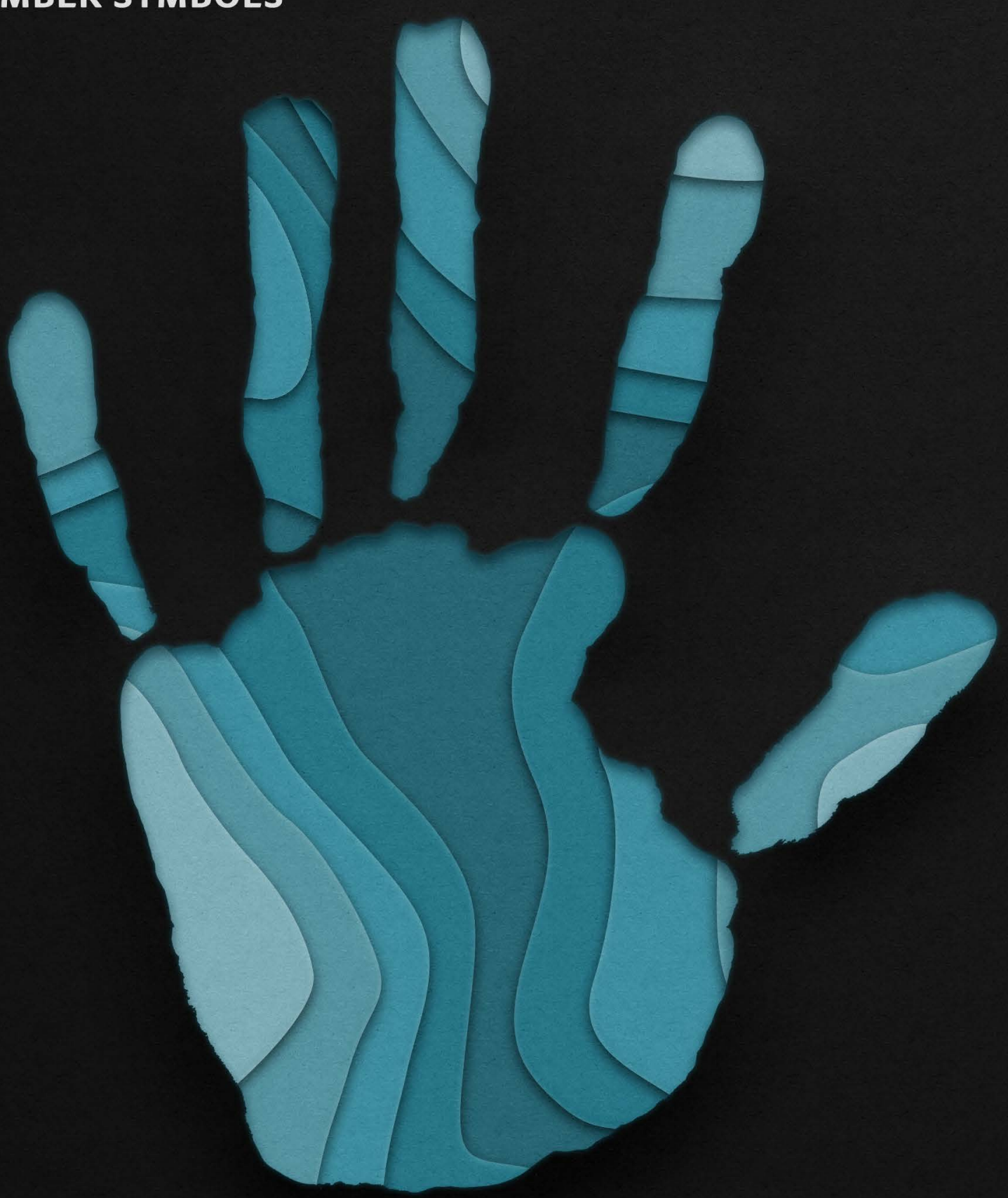




\section{Does Size Matter?}

Behavioral and Neural Insights into the Representation of Small and Large

Number Symbols

Fabian van den Berg 


\section{Doctoral Thesis, MAASTRICHT UNIVERSiTy}

(C) Fabian van den Berg, Maastricht 2021

All rights reserved. No part of this publication may be reproduced, stored in retrieval system, or transmitted in any form or by any means, electronic, mechanical, photocopying, recording, or otherwise, without prior written permission of the author.

Cover and Formatting: Fabian van den Berg

Poster: Sarah Crawley I www.sarahcrawleyvisuals.com

Production: ipskamp Printing | www.ipskampprinting.nl

ISBN: 978-94-6423-556-2

First release, December 2021 


\section{Does Size Matter?}

Behavioral and Neural Insights into the Representation of Small and Large Number Symbols

\section{Dissertation}

To obtain the degree of Doctor at Maastricht University,

on the authority of the Rector Magnificus, Prof. Dr. Rianne M. Letschert,

In accordance with the decision of the board of Deans,

to be defended in public on Tuesday the $14^{\text {th }}$ of December 2021 , at 13:00 hours

by

\section{Fabian Christopher Gerardus van den Berg}




\section{Promotors}

Dr. L. M. Jonkman

Maastricht University

Prof. dr. P. de Weerd

Maastricht University

\section{Assessment Committee}

Prof. Dr. M. Bonte (chair)

Maastricht University

Dr. V. Crollen

Université Catholique de Louvain

Dr. F. C. L. Donkers

Maastricht University

Prof. Dr. E. H. Kroesbergen

Radboud Universiteit Nijmegen 



\section{TABLE OE \\ contzNIS}

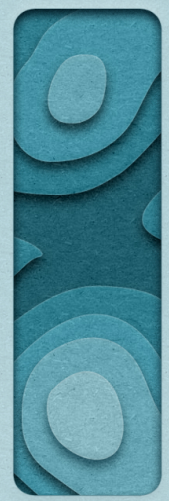

1 General Introduction

2 Numerical Brain Potentials in a Number Learning Task . . . 67

3 Number Representations in children's subitizing range ... 97

4 Internalized Canonical Finger-Number Gestures

5 Finger-Numbers Facilitate Arabic Digit Processing . . . . . 169

6 General Discussion ........................... 203

A General Summary ............................... 233

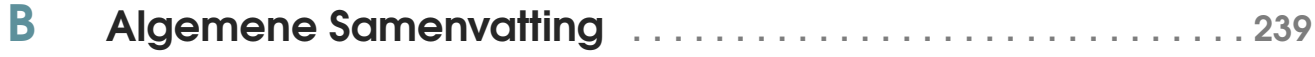

C Impact of this Research ...................... 245

D Acknowledgements ........................ 249

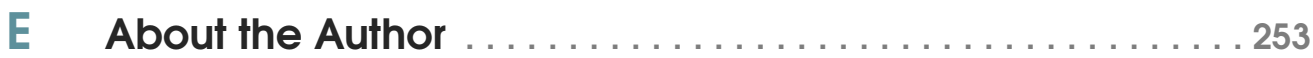





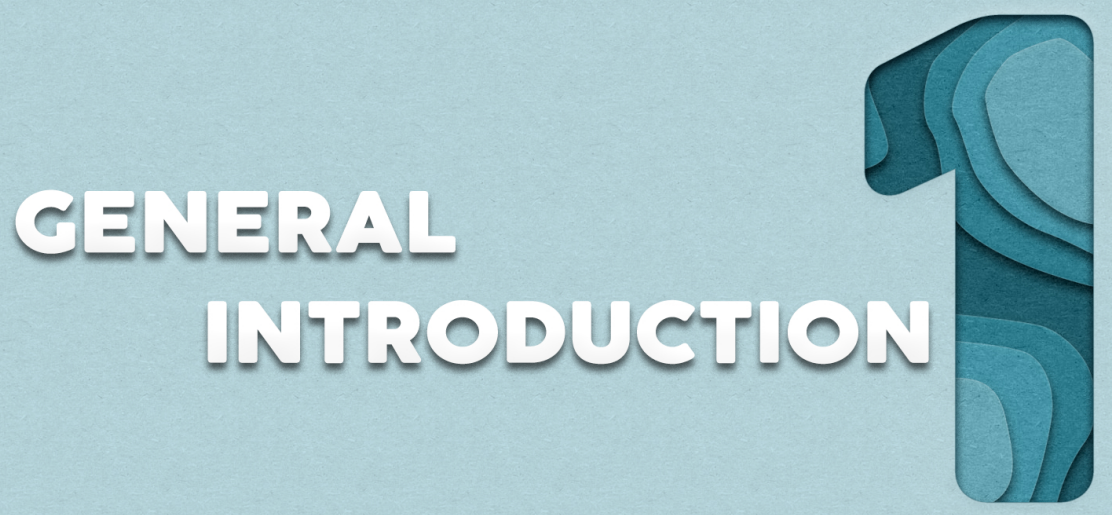

We live in a numerical society; our lives revolve around numbers. Without numbers, we would not be able to do our jobs; we need to be able to keep track, calculate, or express quantities. Our daily life outside of work is also unimaginable without numbers; they inform us how much money we have to spend and when the bus might arrive; from reading a house-number to calculating a pyramid's surface, numbers describe our world. However, as common as numbers are, our numerical skills are not completely innate. They stem from a uniquely human ability to learn and use abstract words or symbols, such as 'three' or '3', as representations of quantities. Once learned, these numerical symbols allow humans to write down an exact amount and communicate it to someone else, a simple but powerful ability in the modern world. However, how numerical symbols gain their meaning is still largely unknown (Harnad, 1990, 2003; Leibovich and Ansari, 2016b).

The current thesis project's central aim was to gain insight into how meaningless symbols (like Arabic digits' 3' or '6') gain their numerical meaning and how they are represented or processed in the brain. The dominant view has been that number symbols (such as Arabic digits 3 or 7) gain their numerical meaning by connecting them to existing non-symbolic representations of quantity (real-world item-sets such as $\bullet \bullet$ ) that are assumed to be present from birth (Dehaene, 2011; Feigenson et al., 2004; Piazza, 2010). The first two studies in the thesis (described in Chapters 2 and 3) used different study designs and paradigms to test this symbol to non-symbolic quantity mapping account. The behavioral and Event-Related brain evidence described in the first study in Chapter 2 showed that in 
a trial-and-error learning task, young adults learned to map novel, abstract symbols onto non-symbolic quantity information, but only for numbers 1, 2, 3, and 4. For novel number symbols 6-9 a much slower learning process took place, challenging the non-symbolic mapping account, at least regarding the learning of number symbols higher than four. In Chapter 3, the validity of the non-symbolic - symbolic mapping account was tested using a longitudinal study design in which behavioral and electrophysiological data from symbolic and non-symbolic number comparison tasks was collected from children twice; the first time when the children were in Kindergarten and one year later when they were in Grade 1. The performance of children was also compared cross-sectionally to the behavioral and event-related potential data collected from adults performing the same tasks. We found behavioral effects related to number processing in both tasks for the small (1-4) and larger (6-9) number range. However, neural effects related to number processing were only present in the small (1-4) number range in symbolic and non-symbolic comparison tasks. The results are discussed in Chapter $\mathbf{3}$ where we suggest different developmental pathways for the symbolic and non-symbolic number system in the smaller and larger number range, also challenging the existing mapping account.

A second aim of this dissertation was to investigate the semantic processing of culture-specific (canonical) finger-number representations in adults. The literature (Andres et al., 2008; Di Luca and Mauro, 2011) proposes that canonical finger-number provide faster and more accurate access to their numerical meaning through stronger semantic associations with internal number representations that are stored in long-term memory. In other words, canonical finger-number patterns are embodied. In addition, we also look at the possible facilitating effects of these canonical finger-numbers on symbolic number processing (Arabic Numerals) and how this might differ between the small (1-4) and large (6-9) number range. The two ERP studies described in Chapters 4 and 5 addressed this aim. We used two different task paradigms to investigate whether there are still signs of culture-specific finger-number representations influencing (facilitating) the processing of other learned number symbols (Arabic digits) in the young adult's brain. Such evidence would point to finger-based number representations being embodied and might signify their potential importance during early number symbol acquisition. Acquiring knowledge of factors that might facilitate or promote number symbol acquisition and processing is important, because better early number knowledge predicts better future math abilities (Cai et al., 2018; Duncan et al., 2007; Elliott et al., 2019; Geary, 2011; Libertus, 2019). In addition, better math abilities in childhood positively affect adult socioeconomic status, likely through increasing a person's qualifications for employment and personal finance 
management (Ritchie and Bates, 2013). Hands (representing numeric information on the fingers) could serve as a facilitator in the process to map symbols onto non-symbolic representations (Geary, 2004; Gelman and Gallistel, 1978; Soylu et al., 2018).

The present chapter outlines the basic concepts, assumptions, and theories from the numerical cognition literature, forming the context in which the research reported in this thesis was conceived. First, a short overview is offered of current theories and proposals of how quantity (non-symbolic) and symbolic number information (number words and Arabic digits) are represented (as investigated with behavioral paradigms; section 1.1 and section 1.2). Next, current thoughts and knowledge about how children acquire symbolic number knowledge will be shortly reviewed. The current views on whether non-symbolic and symbolic number representations share the same or separate representational systems will be pitted against each other (section 1.3). Following is a short overview of the current neuropsychological research into numerical cognition (section 1.4) Additionally, the intriguing links between the body and numerical representations will be highlighted, focusing on the role of finger-number representations and how these can shape and support the acquisition of the symbolic number system (section 1.5). Finally, the specific research questions addressed in the studies described in chapters 2-5 will be laid out (section 1.6). 


\subsection{Exact and Approximate Number Processing}

Like color or size, quantity can be seen as a characteristic of a set of items; the melons are green, bigger than your hand, and there are four of them. The ability to quickly process and discriminate quantities makes perfect sense from an evolutionary perspective. Knowing how many predators are chasing you or how much food is available is a huge advantage, on par with seeing the difference between ripe and unripe fruits based on their color. Many animals, including humans, possess an innate sense of number; the ability to discriminate quantities can be found in mosquitofish (Agrillo et al., 2008), lions (McComb et al., 1994), dolphins (Jaakkola et al., 2005), elephants (Plotnik et al., 2019), mice (Reznikova et al., 2019), dogs (Bonanni et al., 2011), and wolves (Range et al., 2014). However, these animal studies show a limit to the number of items that can be discriminated exactly, and that limit seems to be three to four items.

A similar limit in the ability to discriminate and quickly determine the exact quantity of object sets was first noted in humans in 1871 when Stanley Jevons tossed a handful of beans into a dish in the name of science (Stanley Jevons, 1871). He placed a white box in the middle of the dish, and beans would either make it into the box or not. After every toss, he quickly estimated (without counting and "without the least hesitation") the number of beans that made it into the white box. After each estimation, he counted the number of beans in the box and compared his estimate to the real number. He made no mistakes if there were four or fewer beans in the box, but more and more errors between the number of estimated and counted items slipped in for larger numbers of beans. This same phenomenon was later observed in an experimental setting, and was named subitizing, defined as the human ability to quickly and exactly enumerate only the first four quantities without counting (Kaufman et al., 1949). Most of us will recognize this subitizing ability; looking around, we can "just see" two pens or three cars, but we have more problems "seeing" or discriminating five or six things. The exact number of items in quantities higher than four (called the non-subitizing range) can only be determined by algorithms such as counting. Kaufman (1949) discovered this by using a task in which adult participants had to name the number of dots they saw on a projected screen. In this dot-naming task, participants saw a random dot-pattern from one of two sets. The first set consisted of randomly positioned dots ranging from 1-15. The second set of dot-arrays displayed one of twenty patterns ranging between 17 and 210 dots. 
Much like the experiment by Jevons (1871), the accuracy and confidence of the participants' dot-array-size estimations were perfect up to arrays of four dots, but thereafter gradually decreased the larger the arrays became (see Figure 1.1).

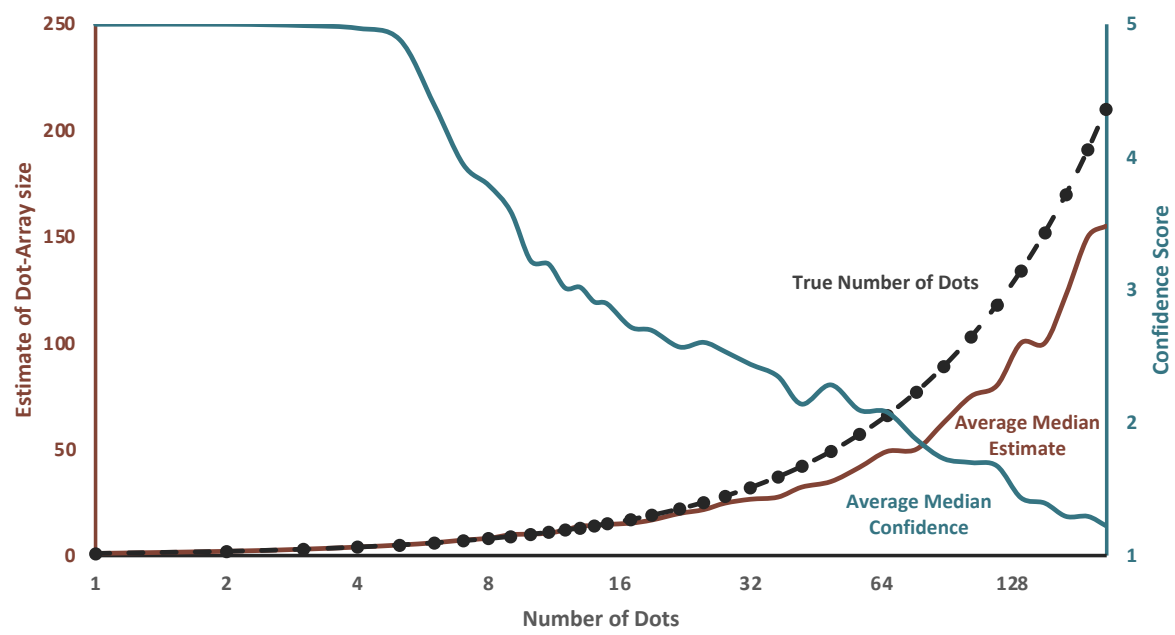

Figure 1.1: Adaptation of results from Kaufman (1949). Data averaged over the speed and accuracy conditions, showing the estimate (solid brown line) and the true number of dots (black dashed line with the patterns used indicated by a black circle), this deviation starts small, leading to reasonably accurate estimates for smaller numbers and a larger deviation with increasing quantities. The confidence in their estimates (solid blue line) shows a strong drop after the subitizing range (after four)

The fact that the first four quantities can be determined with exact precision without counting, but quantities above four cannot, has led to the proposition that two separate numerical systems drive subitizing and estimation abilities. Subitizing has been proposed to rely on a representational system called the Object Tracking System (OTS) to process and enumerate small numerosities up to four items (Feigenson et al., 2004). On the other hand, estimation has been proposed to rely on the Approximate Number System (ANS) involved in determining the size of larger numerosities (Dehaene, 2011). These two systems will be further explained in the next two sections. 


\section{. Box 1.1}

One extraordinary example of math-capable creatures is the humble honeybee. Not only do bees grasp the concept of zero (Howard et al., 2018), they can also learn basic addition and subtraction (Howard et al., 2019). The researchers trained the bees to recognize colors as signs for addition (blue) or subtraction (yellow) through conditioning. Once the bees had associated addition and subtraction to these colors, they were then put in a Yshaped maze with two paths, left and right. At the beginning of the maze, they would always see a sign with three squares; the correct answer would be either four or two, depending on the squares' color (blue or yellow). At the split in the path, the bees would see two signs using the same color as the sample, showing the correct and incorrect answer on either side. The incorrect answer was in the same direction as the sample (five or one) or in the other direction than the color indicated (two or four). When they flew to the correct answer, a sweet reward waited for them, while the incorrect path led to a disgusting liquid. After some practice, the bees made the correct choice by flying towards the sweet liquid $64-72 \%$ of the time, well above chance level. This result does not mean bees fly around doing math; it is highly unnatural behavior for them. It does show that they are capable of learning, making a good case for the systems that support these abilities to be very old from an evolutionary perspective.

More natural examples of the ability to discriminate small or large quantities can be found in studies with wild animals in their natural habitat. In a study with lions, McComb et al. (1994) played recordings of encroaching strangers, closely observing how the pride would respond. When there was only one intruder, the lions would take on an aggressive stance, ready to defend their territory. If the recording played the sounds of three intruders, the lions took on a more cautious approach, and, in some cases, they went back to recruit more group members. A second example comes from an animal that is a bit closer to humans, the Chimpanzee. Chimpanzees live in social groups, called communities, that require them to hold and defend their territory not to lose precious food to neighboring rival communities. Chimpanzees patrol their borders and do not shy away from conflicts with intruders, but they will patrol more silently, slowly, and less aggressively based on the number of outsiders they hear (M. L. Wilson et al., 2001). 


\subsubsection{The Object Tracking System: Representation of small quantities}

As explained in the previous paragraph, small quantities up to four items are subitized; we can immediately and accurately enumerate or discriminate quantities of up to three or four items without using counting strategies. Since the phenomenon of subitizing was first observed by Jevons (1871) and Kaufman (1949), multiple studies replicated it (Mandler and Shebo, 1982; Trick and Pylyshyn, 1994). Trick \& Pylyshyn (1994) proposed that subitizing depends on a system that represents and tracks individual objects called the Parallel Individuation (PI) or Object Tracking System (OTS; See Figure 1.2; Pylyshyn, 1994; Pylyshyn and Storm, 1988). The OTS represents individual objects through time and space, serving more functions than just quantity representation. According to Spelke \& Kinzler (2007) and Piazza (2010), these functions include the perception of cohesion (objects that move together belong together), continuity (objects move on a straight path), and contact (objects do not affect each other from a distance). These functions all rely on the OTS and its ability to represent and track multiple individual objects. However, there is a limit to the number of objects the OTS can process or track simultaneously, and that limit is three or four items (Piazza, 2010). Notably, if subitizing works by representing individual objects, rather than representing the set as a whole, it would rely heavily on attention and working memory. This is supported by research showing that both attention and working memory capacity (Cowan, 2001; Leslie et al., 1998; Scholl, 2001) and the subitizing range (see studies by Jevons (1871) and Kaufman (1949) above) are limited to three or four items.

The ability to subitize is present at birth or develops rapidly after (Feigenson et al., 2004). Ross-Sheehy et al. (2003) showed 4-13-month-old infants a slide-show of 1-6 colored squares rapidly appearing and disappearing. In some trials, the color of a randomly chosen square would change, whereas this color change was absent in the remaining trials. The hypothesis tested was that should the infants represent the squares individually, they would look longer at the changing stream; otherwise, they were expected to show no preference. The results indicated that the youngest infants (4 months) showed preferential looking towards changing stimuli only if there was 1 square. In contrast, the oldest (13 months) showed preferential looking towards the changing stimulus for up to 4 squares. 


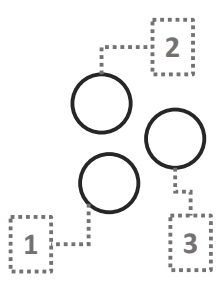

OTS

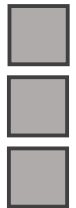

Memory and Attention Capacity: 3

Exact individual representation

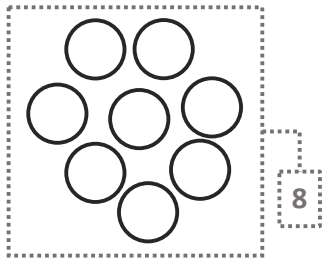

ANS

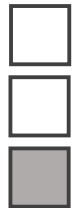

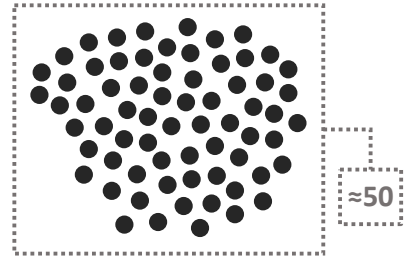

ANS

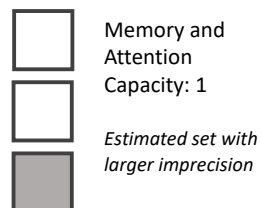

Figure 1.2: Representation of the different ways numbers are represented in the brain. The OTS (left) represents up to three or four items individually as mental object-files, able to track them through time and space. Subitizing would be the extraction of the current number of mental objects represented. The ANS (center and right) represents a whole set, ascribing it a single estimated quantity which becomes increasingly imprecise with larger sets. Medium sized sets (center) can still be represented exactly using algorithms such as counting , whereas larger sets (right) often take too much time and effort to count and are estimated.

These results suggest a fully operating OTS by the age of 13 months. Feigenson et al. (2002) took a different approach when demonstrating the early presence of subitizing in ten and twelve-month-old infants. They put crackers into two separate boxes one by one, after which the infants had to choose one of the boxes by crawling towards it. The infants could see the researchers hide the snacks, and the majority crawled towards the box containing the most crackers $(80 \%)$. Infants consistently chose the box with the most crackers when there were 1 vs. 2 or 2 . vs. 3 crackers in the boxes. The infants' choices became less consistent when one of the options was more than three ( 3 vs. 4, 2 vs. 4, or 3 vs. 6), with only 40-50\% choosing the box with the most crackers. In another study, Feigenson and Carey (2005) hid small balls inside a box, after which 12-month-old infants had to retrieve the balls. They compared how long the infants searched the box within a ten second period after the box should be expected to be empty (e.g., place one ball and then retrieve it) versus how long they searched when balls could still be expected to be in the box (e.g., place two or three balls, and retrieve only one). The infants continued searching after taking out one ball, for up to three balls originally placed in the box. However, when the researcher put four balls inside the box, the infants stopped searching after retrieving the first, suggesting that they had not formed the same exact representation of four as they did for three. 
In summary, subitizing is thought to be a process served by the OTS, a cognitive system responsible for various other visual and attentional abilities. Subitizing is, therefore, constrained to the memory and attentional limits of the OTS, resulting in a maximum of three or four items to be represented in working memory at the same time. Subitizing is also an ability that is present early on and does not require formal teaching, as shown in studies with young infants who develop this ability quickly after birth. Quantities larger than four cannot be represented by the OTS and can thus not be subitized, but have to be estimated which has been proposed to occur via the so-called Approximate Number System (Dehaene et al., 1998; Gallistel and Gelman, 2000), which will be discussed in the next paragraph. 


\subsubsection{The Approximate Number System: Representation of larger quantities}

The Approximate Number System (ANS) represents an object set's magnitude with a rough, non-verbal estimate of its quantity (Dehaene et al., 1998; Gallistel and Gelman, 2000). Whereas objects are represented individually during subitizing, a summary estimate of the entire set is made in the ANS, a single magnitude to represent the quantity of the entire group (Dehaene, 2011). As was mentioned before, when estimating the size of larger quantities, the estimate's imprecision increases (Dehaene, 2003; Dehaene and Changeux, 1993; Dehaene et al., 2008; Kaufman et al., 1949; Stanley Jevons, 1871). In later, so-called magnitude comparison tasks, participants were asked to compare two dot-arrays and decide which of the two was larger or smaller in magnitude. The results of these studies showed that comparison responses were slower and less accurate when the two dot arrays were closer together in magnitude (See figure 1.3). The increasing imprecision in quantity estimations of larger item sets in these comparison tasks resulted in a characteristic effect referred to as the Number Ratio Effect (Buckley and Gillman, 1974; Dehaene, 2007, 2011; Feigenson et al., 2004), based on the discovery that the decision of which of two sets is larger depends on their ratio rather than their absolute magnitude difference. For example, a difference of four items is easy to see when comparing sets of 10 and 14 items (ratio 5:7), but less clear when comparing sets of 24 and 28 items (ratio 6:7). When we have twice as many dots (28 instead of 14), we'd also need to double the distance to get the same performance, known as Weber's Law.

The discovery of the number ratio effect led Dehaene (Dehaene, 2001) to the proposition of the ANS representing a quantity as a gaussian curve, with the peak of the curve denoting the preferred numbers (see Figure 1.4). These activation curves are in line with a form of 'number tuning curves' reported in electrophysiological research in monkeys, which I will discuss in section 1.4. The curve for "four" represents neurons that would fire maximally when viewing a set of four items. It tapers off to the sides representing the partial activation of neurons that prefer three and five (see Figure 1.4). These activation curves also provided a good explanation for the Ratio Effect and its symbolic counterpart the Number Distance Effect (NDE; see Figure 1.3) since units with overlapping curves could interfere with each other. Seeing a set of four and five would lead to two overlapping activation curves, making it slightly more difficult for the brain to decide the quantity of each. Seeing a four and an eight would also lead to two activation curves, but with much less overlap, making it easier to discriminate these numbers. 

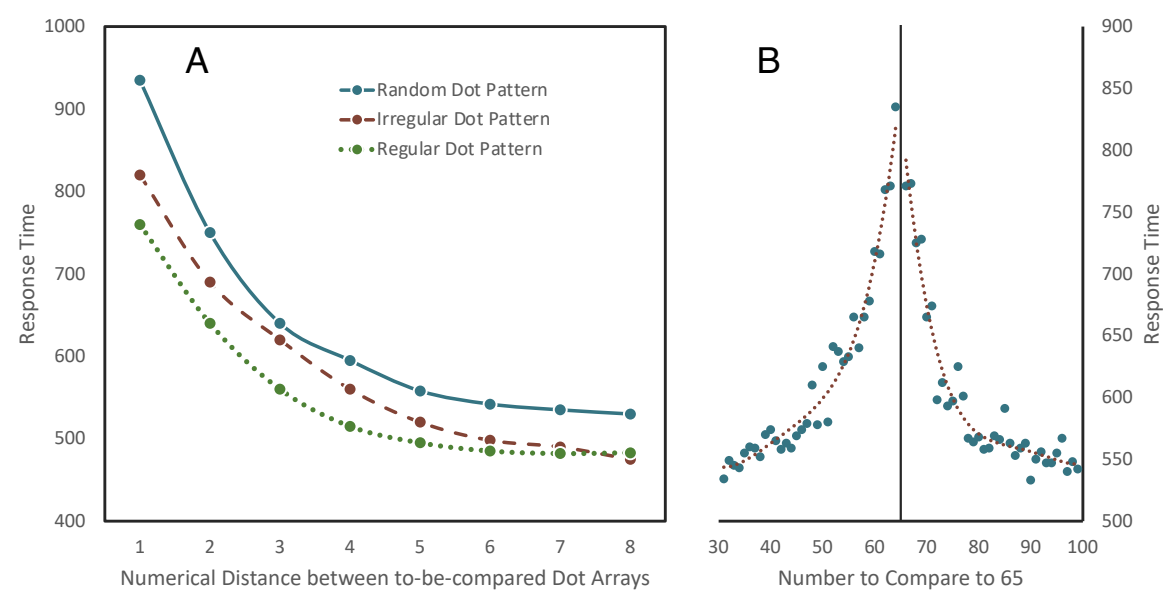

Figure 1.3: Results adapted from Gillman (1974) and Dehaene et al. (2011; 1990). (A). The numerical ratio effect in a non-symbolic number comparison task adapted from Buckley and Gillman (1974), and (B) the number distance effect in a symbolic comparison task using an internal referent as comparison number; adapted from Dehaene et al. $(2011 ; 1990)$. Both display an increase in response time when the number to be compared is numerically closer to the referent.

Similar to subitizing, estimation is also assumed to be present early in development, as indicated by the presence of ratio effects in newborns (albeit only with large differences in smaller set sizes; Barth et al., 2003; Izard et al., 2008; van Oeffelen and Vos, 1982). Izard and colleagues (2009), for example, tested 50-hour old infants, letting them listen to four or twelve sounds while at the same time looking at two pictures of four or twelve shapes. The newly born infants looked longer at the picture that showed the same number of shapes as the number of sounds they heard. Note that the infants did not know they were hearing and seeing four or twelve things; they performed rather a "few versus many" discrimination. The authors concluded that the infants could discriminate numerosities across modalities, matching the number of sounds to the number of shapes. At six months old, the ratio required to discriminate large numbers is about $1: 2$, growing to $2: 3$ at ten months of age, and reaching 7:8 by adulthood (Barth et al., 2003; van Oeffelen and Vos, 1982). 


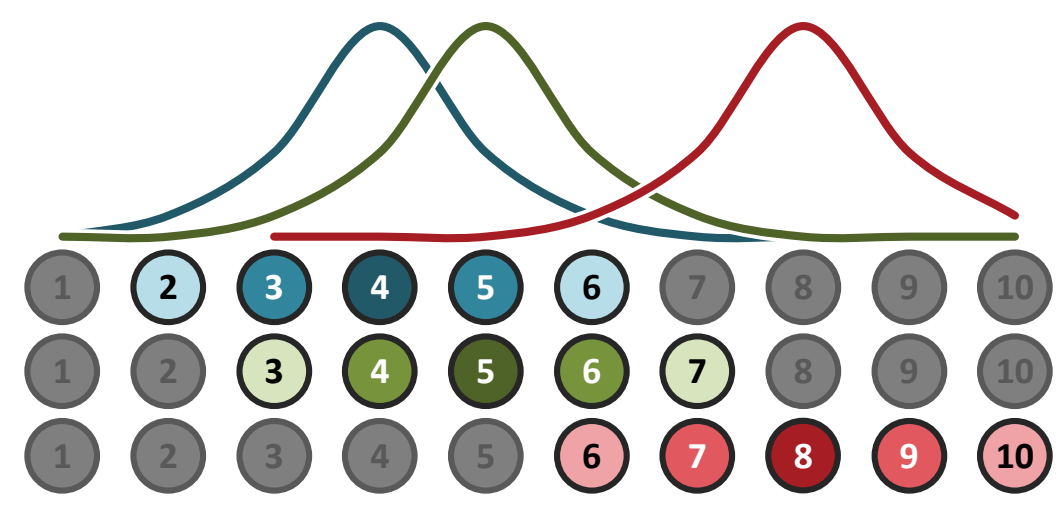

Figure 1.4: Representation of the Number Distance Effect. The Number Distance Effect shown in terms of activation curves. The two curves for four and five partially overlap, there are multiple quantities they 'could' be and four and five have a lot of those possible quantities in common. This overlap between the two quantities causes interference when comparing them, leading to longer response times and more errors when determining the quantities. A comparison between four and eight leads to a smaller distance effect since the overlap between them is less.

In summary, the ANS estimates the quantity of larger sets of objects, creating a single representation. The estimate's precision decreases with increasing size of the set; small sets of 7 or 8 items are estimated reasonably accurate but sets of 700 or 8000 less so. The increasing imprecision of ANS representations results in the characteristic Number Ratio Effect, with decreased magnitude discrimination performance (e.g., slower reaction time and lower accuracy) when the ratio between two to-be-compared non-symbolic quantities decreases. Magnitude comparison tasks have since their first use been frequently applied to investigate ANS development, with the presence and acuity of Number Ratio Effects respectively taken as evidence for ANS usage and development. These studies have revealed that a basic form of the ANS (only being able to discriminate quantities differing with a 1:2 ratio) is already present from birth in humans and in many animal species. However, in humans, the acuity of the ANS develops further across childhood (Halberda et al., 2008; Libertus and Brannon, 2010; Piazza, 2010; Piazza et al., 2010). 


\subsection{The Symbolic Number System and its development}

In addition to the OTS and ANS, educated humans also acquire the Symbolic Number System (SNS), a system of abstract representations of numbers. Number symbols, such as the word "three" or the digit "7," are referents to quantities, but they are arbitrary and do not convey any numerical information in themselves. Unlike the early presence of subitizing and estimation systems discussed above, the SNS is not available from birth but has to be acquired through experience and education (Carey and Barner, 2019; Fuson, 2012; Wynn, 1990). How at first arbitrary symbols might gain their numerical meaning is the subject of much debate and one of the main questions in the current thesis. I will further elaborate on these views and debate in the following section 1.3: Current Views on the acquisition of the SNS. Before entering this debate, the present section briefly reviews current theories on how children acquire the SNS.

The acquisition of the SNS follows a predictable path, starting in early childhood with the learning of number words ("one, two, three"). It does not take children long to learn how to count; at two years of age, children can already count to ten, maybe even further (Wynn, 1990, 1992). Many parents or those familiar with children this age will recognize how they eventually stumble and skip numbers when reciting the number word list, they know the words, but they have not yet acquired the numerical meaning of those words. The lack of young children's understanding of number words was shown in a study by Wynn (1990, 1992). She used a so-called Give-a-Number task in which children handed over a certain number of objects to a puppet (for example, three stuffed animals). At two years of age, most children were "grabbers," meaning they handed over a random number of objects or continued giving objects until given some confirmation that they could stop. These results showed that even though two-year-olds can use number words to count to ten, and have learned that the words have a certain order (ordinality understanding Fuson, 1988), they do not yet understand that the word 'two' represents two objects in the external world; they lack cardinality understanding.

Children start developing their understanding of numerical meaning with the first four numbers. They learn these four numbers one-by-one, followed by an understanding of numerical concept that extend to every other number after four. Conventially children master the numerical meaning of numbers when they are about 3.5 years of age (Condry and Spelke, 2008; Wynn, 1992). Children will start as a pre-or zero-knower, having no understanding of the numerical meaning of words at all. They then proceed to the 
'one-knower' stage, meaning they have a numerical understanding of the concept of "one." In the Give-a-Number task, these children can consistently hand over one object from a pile of objects when asked to "Give me one" and fail to do so with two, three, or higher (Sarnecka and Lee, 2009; Wynn, 1990, 1992). After becoming a one-knower, children proceed sequentially to develop into two-, three-, and sometimes four-knowers over an approximately 18-month period. However, individual variation and overlap exist in the ages at which a child is a two- or three-knower, depending on vocabulary skills and possibly socioeconomic status (Sarnecka et al., 2015).

Once children understand the concept of "four," they gain the insight that the last word reached when counting refers to the total number of items in the entire set of objects; they have gained understanding of the cardinality principle (Wynn, 1990, 1992). Cardinality principle understanding is a milestone for children since it enables them to apply the various patterns, rules, and principles they already know from the wordlist to further develop the symbolic number system and acquire representations of numbers higher than 4 (Carey, 2009; Carey and Barner, 2019). One of these principles is the $n+1$ rule or the successor principle, referring to understanding that each next number word in a sequence refers to a set of items that is one more than the current word. Another is the "later-is-greater" principle, referring to the understanding that words that come later in the list refer to bigger numbers.

The common assumption is that once children have acquired cardinality understanding they have connected number words and later symbols (such as " 3 ") to their mental number line, a language-independent analog representation of magnitude (Dehaene, 2011). We can see the mental number line as a representation where quantities reside next to each other. As described by Dehaene (Dehaene, 2011; Dehaene and Brannon, 2011), this mental number line is the abstract concept of quantity that we access whenever we hear, see, or feel any type of quantity. In this explanation of the mental number line, reading the number " 6 " activates the same concept as hearing the word "six"; they are different representations connected to the same abstract numerosity.

The processing and representation of number symbols on the mental number line is subject to development, which is shown by research using so-called number placement tasks in which one has to position numbers on a line with anchor points at the extremes of the line (e.g., from 0-100). Using such tasks in adults and children from the second, fourth, and sixth grades, Siegler \& Opfer (2003) showed that children and adults relied on different numerical representations. Whereas children showed logarithmic shapes of 
number representation on the number line (from 0-1000), adults' number line representation followed a linear shape, pointing to higher representational acuity in adults (see Figure 1.5). As children grow older and acquire a better understanding of the SNS, also their number line representation gets a more linear shape (2003), leading to more accurate number discrimination performance. Several studies have shown that improvements in number line acuity are associated with improvements in other numerical abilities (Ramani and Siegler, 2008; Siegler and Booth, 2004; Siegler and Ramani, 2009). A study by Matthew \& Chesney (Matthews and Chesney, 2011) has related the shift from a logarithmic to a linear representation of numbers to children's understanding of (relative) quantities.
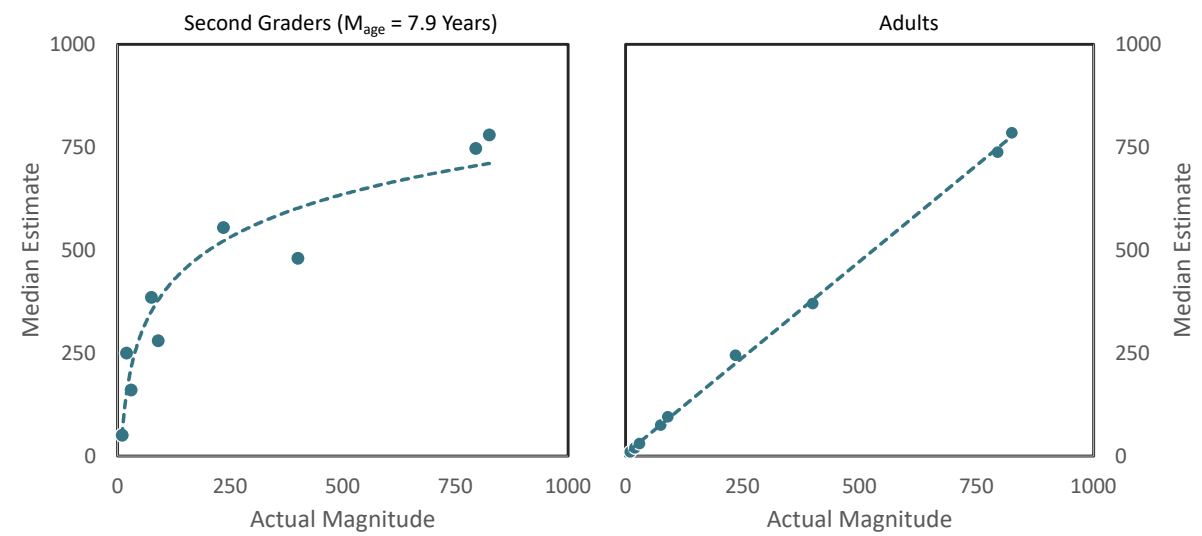

Figure 1.5: Results adapted from Siegler \& Opfer (2003). When performing the Number-To-Position Task (NP) second graders make use of a logarithmic internal representation, leading to logarithmic estimates. Adults performing this task use a linear internal representation, leading to linear estimates.

In summary, the SNS is a learned system that represents quantities as abstract words or symbols. These symbolic numeric representations not only have a connection to a specific quantity, but they also have a connection to each other. Children first learn of these representations as number words, a list without any numerical meaning but with inherent order. Over 18 months, children slowly acquire the numerical meaning of the first four number words one by one until they learn the cardinality principle. It is being argued that the rules and patterns, such as the successor or later-is-greater principle, that are part of the SNS, allow children to generalize what they have learned over an infinite range of possible symbolic numbers once they understand the cardinality principle (Carey, 2009; Carey and Barner, 2019). 


\section{. Box 1.2}

The mental number line is a visuospatial representation of numbers from low to high, left to right. There is evidence suggesting that these spatial number representations are already present early on. De Hevia et al. (2014) habituated 7-month old infants to increasing or decreasing non-symbolic number sequences. The first experiment presented the infants with a left-to-right order of stimuli (so the first shown number, either the smallest or largest, was shown to the left). Regardless of the order they were habituated to, the infants would look longer as the increasing order. The authors interpret this as a spontaneous preference for increasing order (1-2-3 over 3-2-1). They repeated the experiment with a right-to-left presentation, resulting in no preference for increasing or decreasing order. The authors conclude that spatial direction is critical for infants to extract numerical information. It also suggests that there is an innate bias for left-to-right small-to-large spatial ordering of quantity.

The innate bias for the ordering or quantities was also suggested by Rugani et al. (2008), who showed that this might not even be an exclusively human concept. In their study, three-day-old chicks were placed in front of a panel showing five dots, training them to get food by walking around it. After that, they placed the chicks in front of two identical panels showing more or fewer dots than they remember from training. If the panels had two dots, the chicks preferred the left panel $70 \%$ of the time, while the eight dot panels showed a right-side preference in $70 \%$ of the cases. Training the chicks on 20 dots and then placing them in front of 8 or 32 dots resulted in the same behavior. If the chickens saw panels with dots less than the training panel, they would head left. A larger number of dots prompted them to go right. The authors argue that this behavior is strong evidence for a low-to-high left-to-right mental number line that can adapt to determine what is smaller and what is larger than a reference.

Though it might be too speculative to say that infants and chickens understand numerical information or have a pre-determined mental number line, these results do suggest a perceptual bias for a left-to-right small-to-large ordering of quantities. 


\subsection{Current Views on The Acquisition of the Symbolic Number System}

As was discussed above, learning the meaning of the first four number words is a complicated and slow process (Wynn, 1990, 1992). How number-symbols gain numerical meaning has been a point of discussion for years, now known as the "symbol grounding problem” (Carey, 2009; Dehaene, 2011; Le Corre and Carey, 2007; Leibovich and Ansari, 2016a; Piazza, 2010; Reynvoet and Sasanguie, 2016; Siegler, 2016; Spelke, 2017; Sullivan and Barner, 2012). What follows is a short and selective review of some older and more recent views on the symbol grounding in the paragraphs below.

\subsubsection{The ANS Mapping Account}

Dehaene (Dehaene, 2001, 2011; Hersh et al., 1999) first proposed the ANS mapping account, claiming that all numerical representations (quantities, symbols, and words) are mapped onto the same analog representations of number in the ANS. The arguments in favor of this account encompass several observations (for a short overview see Dehaene, 2001). The main observation on which this account relies is the occurrence of seemingly similar numerical distance effects in non-symbolic and symbolic number tasks. In the previous section, I explained the presence of the Number Ratio Effect when comparing two quantities represented by the ANS. Such comparisons are more difficult if they have a larger ratio (e.g., discrimination of 4 vs. 8 dots is easier than 24 vs. 28 dots), resulting in higher response times and lower accuracy. A similar effect occurs when comparing two number symbols (number words or Arabic digits), except that in this case, performance depends on the numerical distance between the two to-be-compared number symbols on the mental number line. If the two numbers are close together, say 4 and 5, the decision which of the two represents a larger quantity takes slightly longer than when the distance is larger (such as between numbers 4 and 8). This effect was called the Number Distance Effect (NDE; Moyer and Landauer, 1967). The Number Distance Effect has been detected in adults (Moyer and Landauer, 1967), infants (Libertus and Brannon, 2010), and even monkeys (Cantlon and Brannon, 2006). Dehaene (2001) argued that the similarity between the numerical ratio and distance effects indicated direct mapping of symbols onto the ANS, eliciting the same overlapping curves as a non-symbolic quantity would, resulting in the same distance effects (see Figure 1.6). 


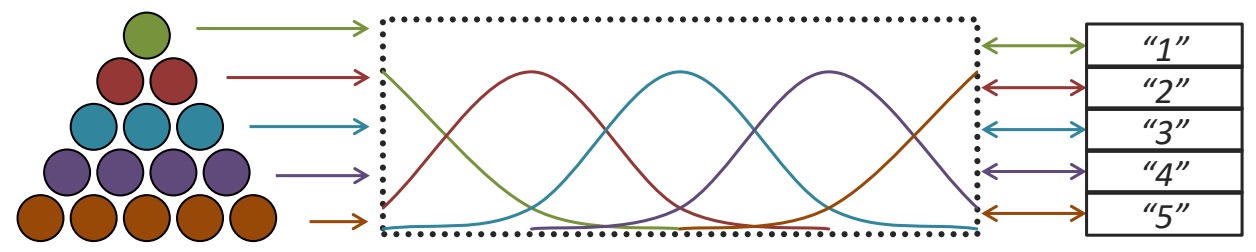

Figure 1.6: Representation of the ANS Mapping Account. The ANS Mapping account assumes symbols are all mapped onto non-symbolic ANS estimates (represented by curves that show a preference for a quantity with a range of uncertainty to each side). The symbols take on the same characteristics of these estimates and therefore show the same numerical distance effects.

\subsubsection{The Rebuttals to the ANS Mapping Account}

The ANS Mapping account remained the dominant view on the symbol grounding problem for several years. However, in their review paper, Reynvoet and Sasanguie (2016) concluded it is unlikely that the symbolic number system gains its meaning through one-to-one mapping onto the ANS, at least not completely. They pointed out that numerical distance effects on response time in number symbol comparison tasks (e.g., NDE) can also result from other aspects of the symbolic number system, such as the relationship numbers have to each other (through language). These semantic distance effects were shown by Chiao et al. (2004). In their experiment, participants compared number words (ranging from 33 to 99 with the referent of 65) and non-numerical ranks. In the first experiment, participants compared University ranks (secretary, Dean, Assistant Professor, and Associate Professor, with Assistant Professor being the referent). The results indicated distance effects in both conditions, showing that rankings closer to "Assistant Professor" were more difficult to discern and resulted in slower responses than those further away. However, these effects might have been due to the task's difficulty, so they did a second experiment to confirm the distance effects were due to semantic distance. In the second experiment, insignia badges of military ranks (Lieutenant, Commander, Captain, Fleet Admiral; with Captain as the referent) were used, rather than academic ranks. Participants (US Navy midshipmen) had explicit knowledge of these ranks, more so than the participants in the first experiment had about academic ranks. The same semantic distance effect was found (see Figure 1.7A), and unlike in the first study, this did not correlate with accuracy. The authors conclude that symbolic representations (such as rank insignias) link to an internal semantic representation, showing similar distance effects as those seen by numbers. 

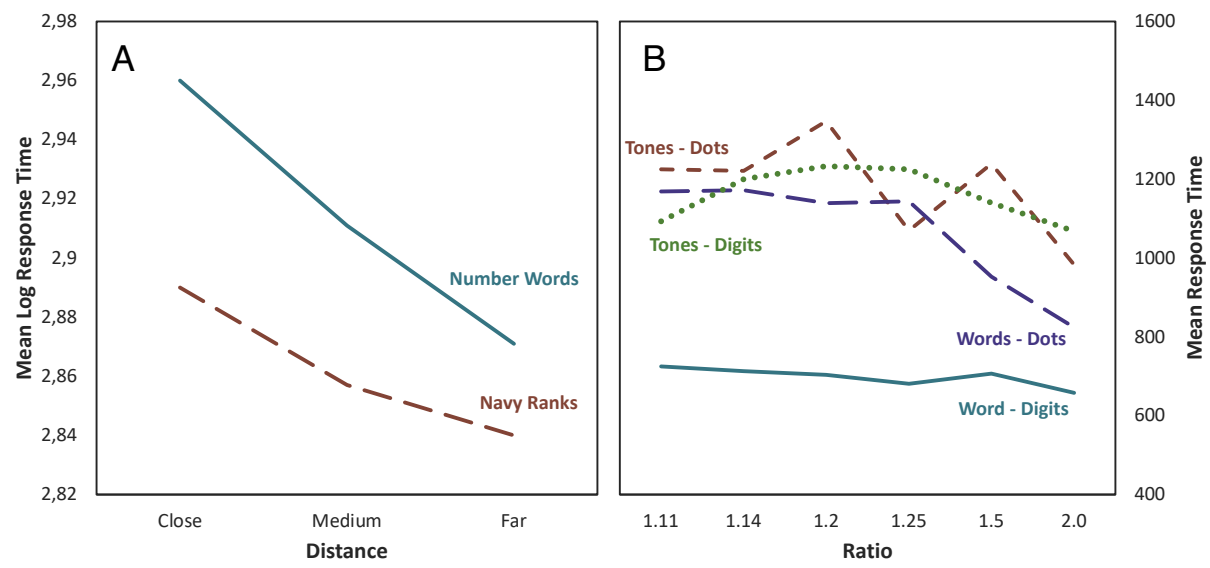

Figure 1.7: A: Results from Chiao et al., (2004) showing semantic distance effects for US Navy Ranks on response times in a Comparison Task with internal reference. B: results from the Audio-Visual Comparison Task in Marinova et al. (2020), showing ratio distance effects only when non-symbolic representations are involved.

Another study by Lyons et al. (2012) also questions the ANS mapping account's assumptions. In their paper, participants visually compared Symbolic and Non-Symbolic representations of numbers (Words, Numerals, and Dots), performing worse when these formats were mixed. They concluded that the SNS and ANS are two separate systems and speculated that the SNS eventually becomes "estranged," meaning that the SNS loses the connection to non-symbolic quantities and only operates as an associative system in adults, using symbol-to-symbol relations. These findings were confirmed by Marinova et al. (Marinova et al., 2021) using a cross-modal number comparison task. Participants in this experiment compared different Non-Symbolic (Tones, Dot-Arrays) and Symbolic (Spoken Words, Arabic Numerals) representations, ranging from 10 to 40. The results demonstrated numerical ratio/distance effects in tasks including at least one non-symbolic number stimulus (Tones + Dots, Words + Dots, Tones + Digits), but not in tasks including only number symbols (Words + Digits; see Figure 1.7B). In addition, there was a switchcost, indicated by slower response times when non-symbolic and symbolic representations were mixed. The authors concluded that this switch-cost indicated the extra time needed to integrate the representations from two systems, and together with the absence of a distance effect in the pure symbolic condition, they concluded that the SNS and ANS were two independent systems. Lastly, Nieder (2020) has proposed, based on the available evidence from animal studies, infant studies, fMRI experiments, and computational modeling, that the Symbolic Number System is partially grounded in the non-symbolic ANS. The learned SNS builds upon the innate ANS capabilities by necessity because it is the only source 
of numerical information. Lyons et al. (2012) suggested that this initial connection is then lost over time, as the relationships between symbols overtake the relations between symbols and their non-symbolic quantities.

An influential alternate model put forth by Carey (2009), later named the Exact Algorithms Hypothesis (Carey and Barner, 2019), was more grounded in previous developmental results. The Exact Algorithms Hypothesis proposes that symbolic non-symbolic mapping does occur but is limited to the lower (1-4) numerical range, where subitizing provides exact and reliable numerical information (see Figure 1.8). Such mapping occurs slowly through the association of symbols to their non-symbolic representation of quantity until children have cardinality understanding of the fourth symbol (as discussed in Paragraph 1.3). For symbols to gain an exact meaning, the OTS provides better numerical information than the estimates of the ANS, so this approach assumes symbols are associated with quantities via the OTS instead of the ANS. According to this model, symbols higher than four are no longer directly mapped onto non-symbolic representations. Instead, these symbols gain their meaning through inference, using language and the symbol-to-symbol relations (algorithms such as the successor principle) rather than via direct non-symbolic mapping (Carey and Barner, 2019). For number symbols higher than four, their place in the number list determines their value without associating them with non-symbolic variants.

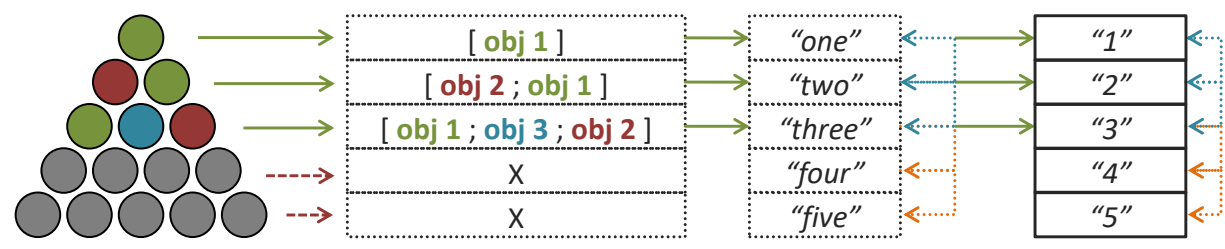

Figure 1.8: Illustration of the Exact Algorithms Hypothesis. The Exact Algorithms Hypothesis limits symbolic to non-symbolic mapping to the subitizing range (indicated by the green arrows between the exact numerical representation and the number words and number symbols). Symbols beyond the subitizing range cannot be represented exactly, instead they gain meaning through inference (indicated by orange lines representing the symbol-to-symbol relationships).

Taken together, different findings in adults and developmental findings argue against the central assumption of the ANS mapping account proposed by Dehaene (2001), that all symbols are mapped onto their corresponding analog representation of numbers provided by the ANS. For example, whereas the presence of similar distance effects in symbolic and non-symbolic number comparison tasks was long assumed to reflect both relying on the same ANS representations, recent studies have shown that distance effects in symbolic tasks can also be explained by the relationships that symbols have with each other 
(Krajcsi et al., 2016). Furthermore, results from several behavioral developmental studies suggest that mapping might differ for smaller numbers 1-4 (subitizing range) and larger numbers (non-subitizing range; Hutchison et al., 2019; Sullivan and Barner, 2014). Recent proposals and findings suggest that only the first four symbols and words directly map onto non-symbolic quantity representations. Beyond four, larger numbers gain their meaning through inferences of the cardinality principle and algorithms such as counting (Carey and Barner, 2019). These larger numbers no longer need to be associated with a quantity in the real world; their meaning comes from their relationship with other numbers (Carey and Barner, 2019). In line with this developmental work, an important aim of the studies in the present thesis was to further investigate the possible different mapping/representation of small (1-4) versus larger (5+) number symbols, by in addition to performance measures, also studying effects of numerical range on event-related brain potential components (e.g., the $\mathrm{P} 2 \mathrm{p}$; see section 1.4 below) that has in prior research been associated with semantic (magnitude) processing of number symbols. This work will be reviewed below.

\subsection{Neurophysiological research into Numerical Cognition}

The previous sections discussed subitizing and estimation, how they relate to two cognitive systems (the OTS and ANS), and the behavioral data supporting these concepts and models. In addition, the Symbolic Number System was introduced; the system most educated adults use to express quantities. In the previous section, two possible accounts were discussed that attempt to explain how abstract number symbols (e.g., verbal and Arabic numerals) gain numerical meaning. However, all or most studies reviewed so far reported behavioral (response time and accuracy) data. It should be noted that response time and accuracy are end-point measures representing the outcome of multiple cognitive processes, from early processes including sensation and perception to later processes including memory and decision making. To gain insight into some of the brain processes underlying the cognition of numerosity, the use of Event-Related brain Potentials (ERPs) can be an interesting tool. ERPs reflect stimulus-processing at a millisecond level and can provide a closer view on the spatio-temporal processes in the brain that occur during the mapping and processing of number symbols. The following sections review first some neurophysiological studies in monkeys shedding light on relevant brain processes involved in numerosity, after which a short review of the ERP-numerical processing literature is offered, focused on links between the numerical distance effect (as an index of analog numerical representation) and the N1 and P2p components. 


\subsubsection{Neurophysiological research on number symbol mapping in monkeys}

As discussed previously, the first step in learning number symbols is to associate them with a numerical value. Diester and Nieder (2007) investigated the neuronal mechanisms of this association by training several Rhesus monkeys to associate the shape of an Arabic Numeral to a dot-pattern quantity for the quantities 1-4. The monkeys performed a trialand-error delayed-match-to-sample learning task with feedback, in which they saw a sample display (a dot pattern) followed by a test display (an Arabic digit) and had to release a lever when both showed the same numerosity. Electrophysiological results from electrodes implanted in the prefrontal (PFC) and parietal cortex demonstrated numberspecific neurons, primarily in the PFC. These neurons responded to specific numerosities, regardless of presentation (as a dot-pattern or a numeral).

In a follow-up study, Diester and Nieder (2010) investigated whether the earlier results reflect monkeys treating these numeral shapes are numerical signs or as visual signs. They expanded the delayed-match-to-sample task into three conditions: Dots vs. Dots, Numerals vs. Dots, and Numerals vs. Numerals. The task was the same as in the previous experiment: the monkeys had to release a lever when the test display was numerically the same as the sample display. The behavioral results indicated Numerical Distance Effects; the monkeys made more errors when the sample and test displays were numerically closer together. The authors interpret these effects as evidence that these responses were driven by the association between the numerical meaning and the numerals, not their visual appearance. This follow-up study strengthens their earlier work, bolstering the conclusion that the number-specific neurons found in Diester and Nieder (2007) reflect a biological correlate of the mental number line. In addition, fewer number-specific neurons were found in the parietal than in the prefrontal cortex, which Diester and Nieder (2007) concluded to be in line with earlier fMRI studies where children and adults had to compare symbols (Ansari et al., 2005; Kaufmann et al., 2006; Rivera et al., 2005). These studies indicated stronger neural activity in the PFC in less experienced human children than adults. Thus, these fMRI studies taken together indicate that as children get older, the activation pattern elicited in number tasks shifts from primarily PFC to the parietal areas, which the authors interpret as reflecting increased number-processing automation. Later fMRI studies had adult humans view random dot arrays of different quantities and found number-related activity in the parietal areas, which showed a topographical organization of quantities. The neurons with a preference for two were physically closer to three than four (Harvey et al., 2013). The studies by Diester and Nieder $(2007,2010)$ show a biological basis for the mental number 
line, with neurons that specialize in numerical information, regardless of their notation. Additionally, they show that monkeys can learn to associate the meaningless visual shapes of Arabic numerals with quantity information and activate those same number-neurons based on their numerical meaning and not simply their appearance.

\subsubsection{ERP correlates of the processing of number symbols or quantities in humans}

Electrophysiological studies in humans investigating ongoing symbolic and non-symbolic mapping processes are unfortunately scarce. However, multiple ERP studies have investigated the neural processing of already learned non-symbolic (e.g., arrays of dots or other stimuli) and symbolic (words and digits) number stimuli in children and adults. In the context of the present thesis, studies that reported on the early perceptual $\mathrm{N} 1$ and the later mid-latency P2 (or P2p) ERP components are especially relevant. Dehaene (1996) performed one of the first numerical processing ERP studies in which adult participants had to decide whether Arabic numerals or Number Words showing quantities 1, 4, 6, and 9 were larger or smaller than 5 while their performance and ERPs were measured. Behavioral results showed the presence of number distance effects for number words as well as digits; participants responded faster to quantities 1 and 9 than to quantities 4 and 6 .

The presence of a number distance effect was concluded to be indicative of both number words and digits being represented/mapped onto an analog, spatial representation of numbers (i.e., the mental number line). Furthermore, correlates of the number distance effect were present in the amplitude of the P2p occurring 220ms after stimulus presentation. Higher amplitudes were observed for comparison numbers closer to five than for those further away, the latter being easier to discriminate from five. Dehaene (1996) concluded that the P2p component reflected the first processing stage in which one has access to the numerical meaning of familiar number symbols regardless of their notation (e.g., words and digits share neuronal representation). Higher $\mathrm{P} 2 \mathrm{p}$ amplitudes were proposed to reflect the recruitment of more cognitive resources when the numerical distance was smaller. Several studies replicated these findings, showing P2p ratio or Number Distance effects in non-symbolic tasks (Rubinsten et al., 2013) in tasks with symbolic and non-symbolic numbers larger than six (Hyde and Spelke, 2009; Libertus et al., 2007; Pinel et al., 2001), and in children (Gouet et al., 2018; Temple and Posner, 1998). 
Whereas Dehaene (1996) did not find numerical distance effects on the earlier exogenous N1-ERP component in his symbolic number comparison tasks, some later studies did report such early distance effects on the N1 in symbolic and non-symbolic tasks (Hyde and Spelke, 2009; Pinel et al., 2001; Temple and Posner, 1998). Further studies, however, revealed that in non-symbolic number processing tasks, the N1 was more sensitive to the visual characteristics of a stimulus than to its numerical value and was accordingly assumed not to reflect semantic number processing. A first study to address visual versus numeric information contributions in non-symbolic number tasks was carried out by Libertus, Woldorff, and Brannon (Libertus et al., 2007). They used symbolic and non-symbolic number comparison tasks to investigate the distance effect on the N1. They extended the study done by Dehaene (1996) and asked participants to compare numbers in the 1-9 range to an internal reference of 5 , deciding whether the shown symbols or dot-arrays were larger or smaller than 5. As expected, number distance effects were found on both the N1 and P2p components, which might have been confounded by visual cues present in the dot-arrays, such as the size of the dots or the total area they occupied in the display. A second experiment was done to remove this possible confound, now only including a non-symbolic task with dot arrays and larger quantities outside the subitizing range (between 8-30 items) and internal reference number 15 . The use of larger quantities minimized the perceptual differences between the differently sized stimulus displays (visual differences between set sizes of 1 and 3 are more salient than between 11 and 13). After controlling for visual cues, distance effects on the N1 were indeed no longer present with these larger stimulus arrays, whereas the distance effect on the $\mathrm{P} 2 \mathrm{p}$ to the non-symbolic stimuli was still present and mirrored the distance effects seen in the symbolic task. These results led to the conclusion that the N1 component is driven by perceptual cues, while the $\mathrm{P} 2 \mathrm{p}$ is driven by numerical information regardless of presentation format (symbolic or non-symbolic). 
In another study by Gebuis \& Reynvoet (2013), participants had to watch nonsymbolic dot-arrays in two task conditions: responding to a color change, and in the other, participants were asked to provide an estimate of the number of dots in the array. When controlling for visual properties of the arrays (such as total surface area or dot-size), no number-related distance or size effects were found on the N1 or P2 components in the color detection or number estimation conditions. The stimuli were created in such a way that the visual cues (such as dot-size) did not correlate with increasing numerosity, and the absence of number related effects would imply that the changes found in previous studies were related to the visual cues rather than to the numerical meaning of the arrays. The authors conclude that visual cues likely confound number-related findings on non-symbolic number tasks.

Only two studies have investigated the modulation of the N1 and P2p ERP components during the symbolic - non-symbolic mapping process in adults by mimicking the learning process. Zhao et al. (2012) had adult participants learn the meaning of nine new symbols through magnitude mapping by showing the symbols paired with nine dot-arrays consisting of 10 to 90 dots (in increments of 10) or through ordinality by showing the new symbols in the correct relative order. The effectiveness of learning was judged based on performance in a subsequent number comparison task. In this task, two new symbols were shown, and participants had to indicate which of the two represented the largest magnitude. The results demonstrated the familiar distance effects on performance measures, with faster and more accurate responses to new symbol pairs that were numerically further apart, indicating that participants had successfully associated numerical meaning (representation on the number line) with these symbols. The ERP results confirmed this conclusion, showing distance effects on the P2p in line with previous literature. The symbol pairs closer in numerical value elicited larger $\mathrm{P} 2 \mathrm{p}$ amplitude, and this numerical distance effect decreased over time as symbols were learned (indicating less cognitive resources were required to retrieve the quantity). In another study, Merkley et al. (2016) also taught participants new symbols (representing magnitudes 20, 27, 36, 50, and 68) using magnitude and ordinality, similar to Zhao et al. (2012) but with the addition of auditory and text labels. Learning was again assessed using a magnitude comparison task, where participants indicated whether a presented symbol (20, 27, 50 0r 68) was smaller or larger than the anchor symbol 36. Also in this experiment, behavioral results showed the expected distance effect, with faster and more accurate responses to symbols further away from the reference. The EEG analysis supported these behavioral results, showing higher mean N1 and P2p amplitudes in response to symbols closer to the referent. 
Concluding, in both Zhao et al. (2012) and Merkley et al. (2016), P2p distance effects in response to novel symbols were detected after learning them, showing that these novel symbols gained an association with a non-symbolic quantity. However, these studies investigated numbers larger than ten that are processed by the ANS and thus would not have gained an exact numerical meaning. This limitation leaves open the possibility that mapping symbols onto non-symbolic representations is different inside and outside the subitizing range (1-4).

Taken together, electrophysiological research has found number-neurons in the parietal and prefrontal cortex, which are sensitive to specific numerosities (Diester and Nieder, 2007, 2010). These studies concluded that initial associations between symbols and numerical meaning are created in the prefrontal cortex and that activity moves towards the parietal areas once symbol processing is automated. The activation of number-sensitive neurons leads to tuning curves, with a preference for a specific numerosity but also responsive to neighboring numerosities to a lesser degree. The width of the tuning curves results in overlap between numerical representations. The Number Distance Effect detected at the P2p ERP- component reflects this overlap, with higher amplitude being associated with more interference and the need for more "effort" or attentional resources to resolve the comparison of two symbols. Numerical effects on the $\mathrm{N} 1$ component, on the other hand, most likely reflect changes in visual cues associated with increases or decreases in non-symbolic quantities. Research investigating neural changes before and after learning new number symbols builds a case for these components being related to numerical processing. The N1 and P2p components are modulated by the acquisition of numerical symbols, learned through magnitude or ordinality information. 


\begin{tabular}{|c|c|c|c|c|c|c|c|c|c|}
\hline & 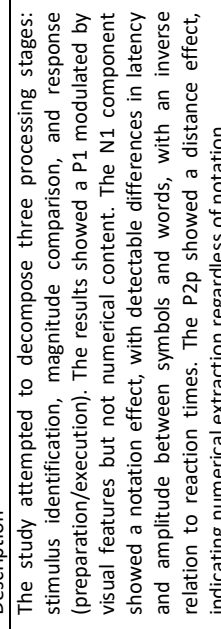 & 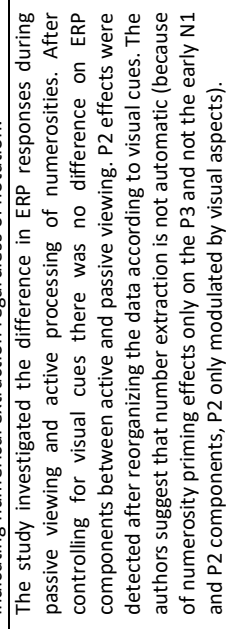 & \multicolumn{4}{|c|}{ 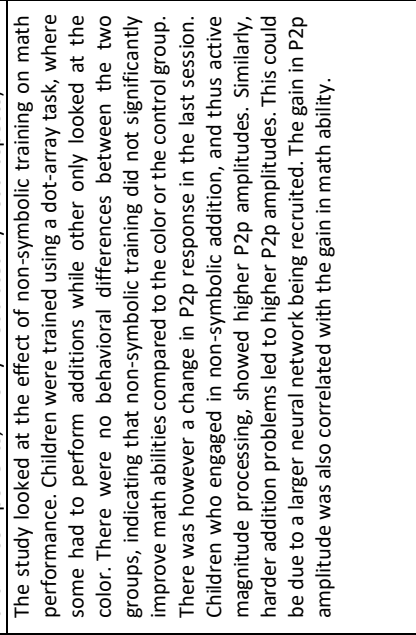 } & \multicolumn{3}{|c|}{ 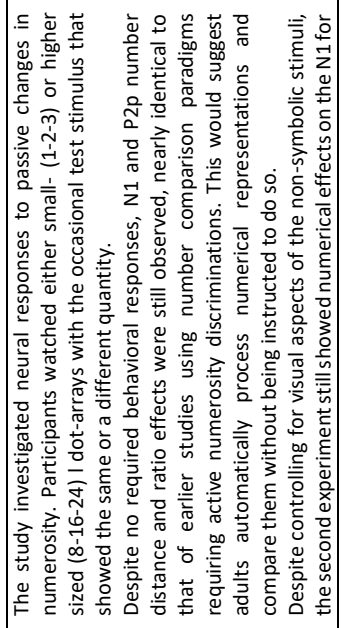 } \\
\hline & 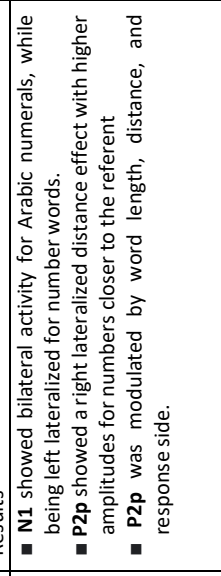 & 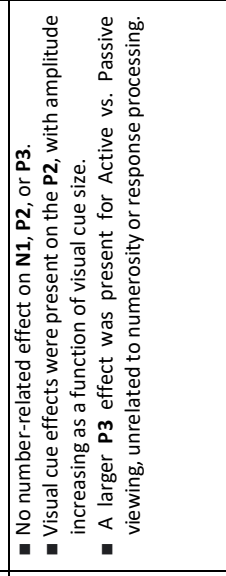 & 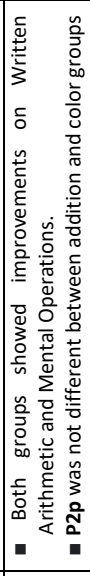 & 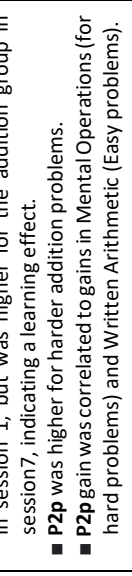 & & & 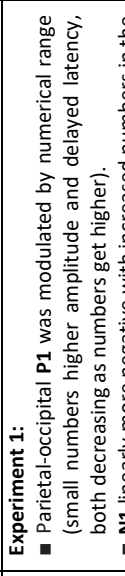 & 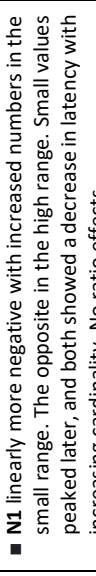 & 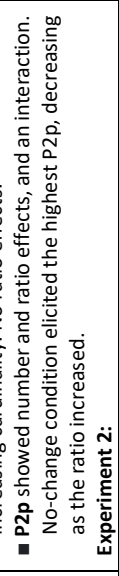 \\
\hline خَ & 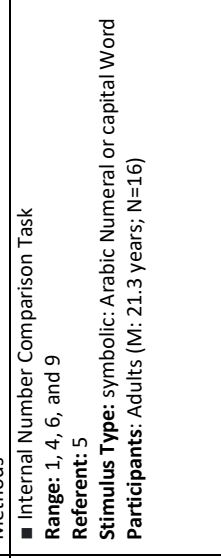 & 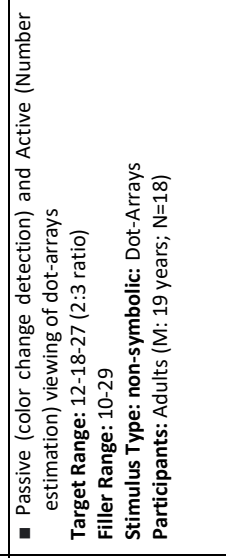 & 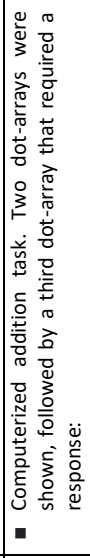 & 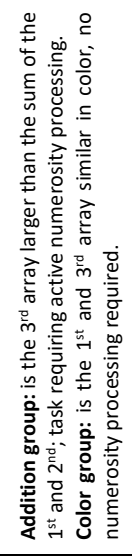 & 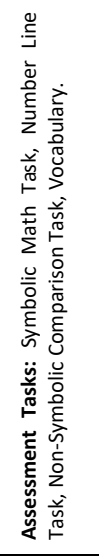 & 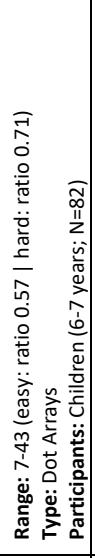 & 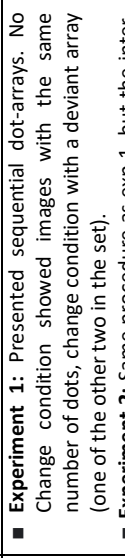 & 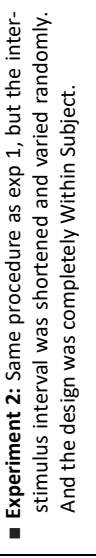 & 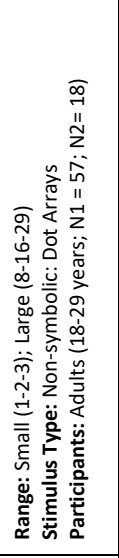 \\
\hline & & 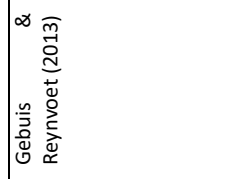 & 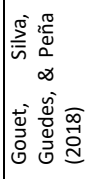 & & & & $\infty_{0}^{\infty}$ & & \\
\hline
\end{tabular}




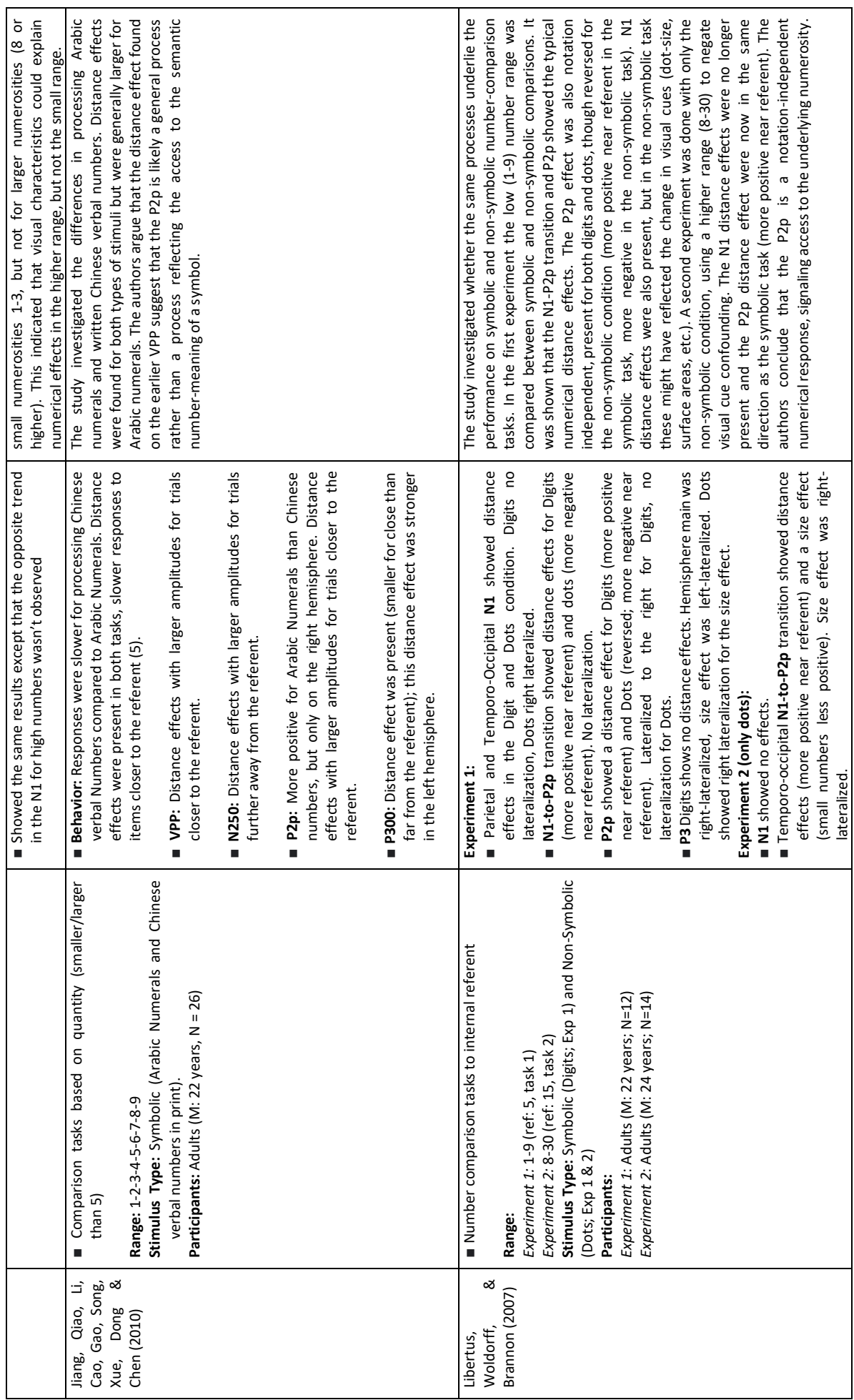




\begin{tabular}{|c|c|c|c|c|c|c|c|c|}
\hline & 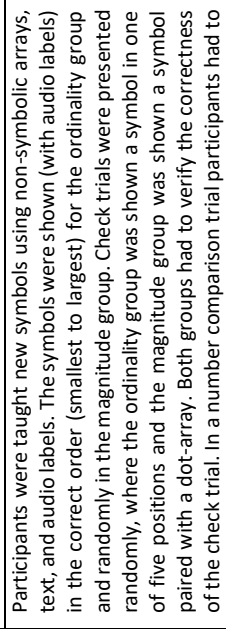 & 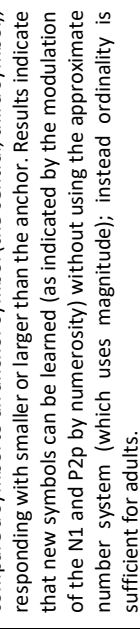 & 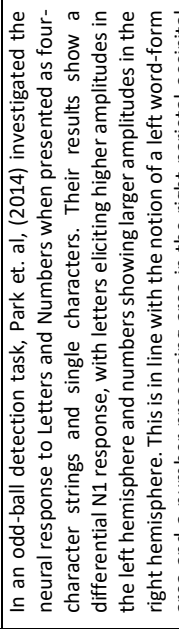 & & 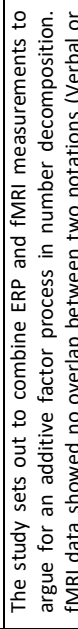 & 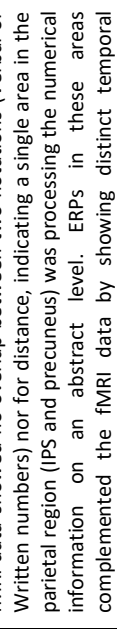 & 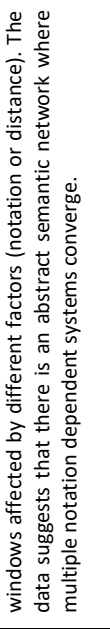 & \\
\hline 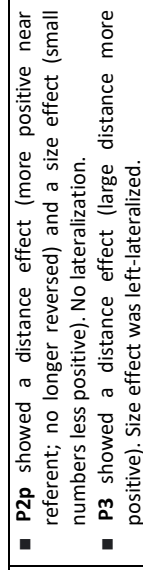 & 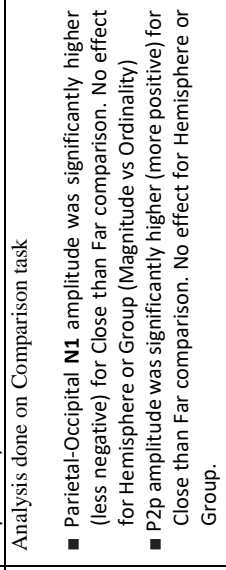 & & 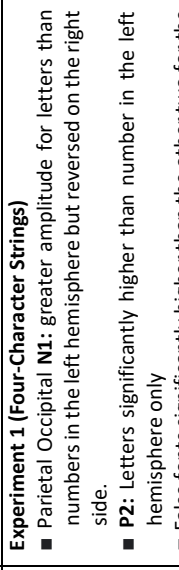 & 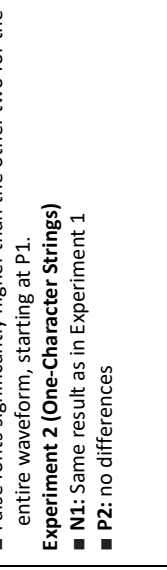 & 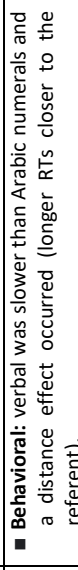 & 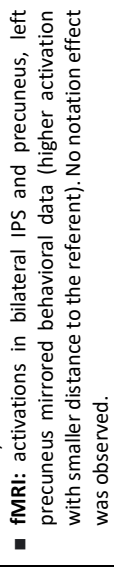 & 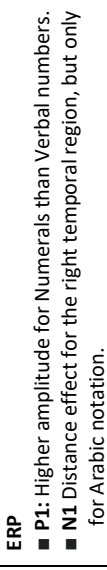 & 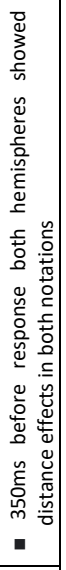 \\
\hline & 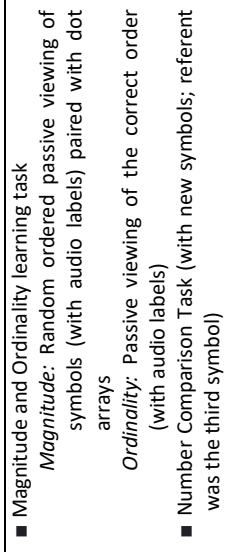 & 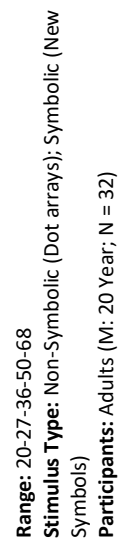 & 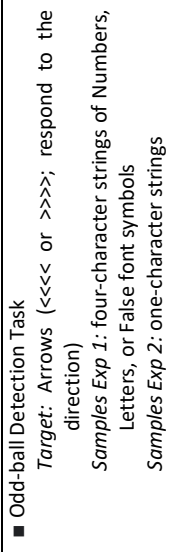 & 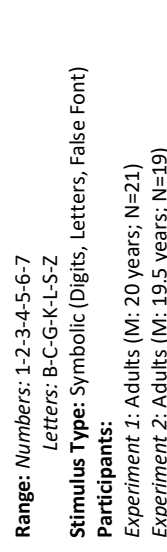 & 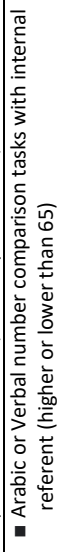 & $\begin{array}{l}n \\
\\
\end{array}$ & 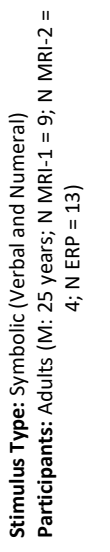 & \\
\hline & 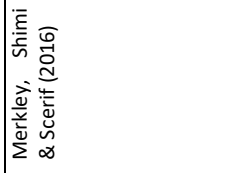 & & 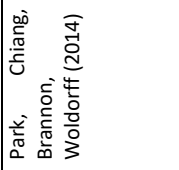 & & 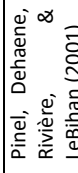 & & & \\
\hline
\end{tabular}




\begin{tabular}{|c|c|c|c|c|c|c|c|c|c|}
\hline \multicolumn{2}{|c|}{ 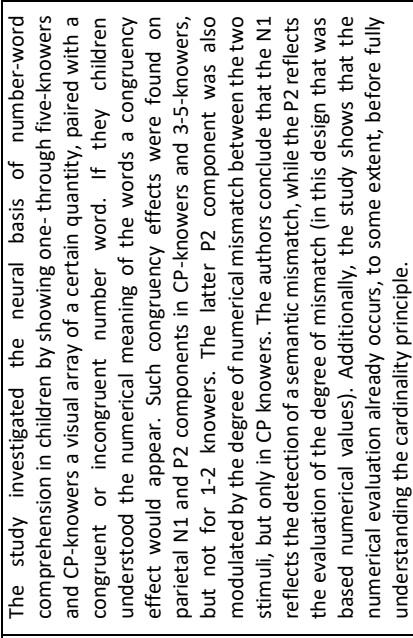 } & \multicolumn{4}{|l|}{ 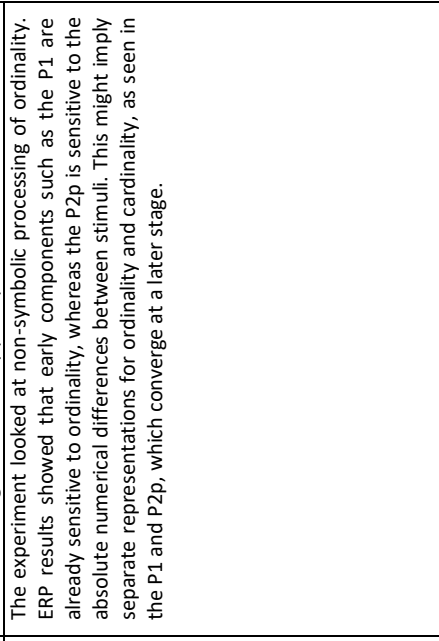 } & \multicolumn{4}{|c|}{ 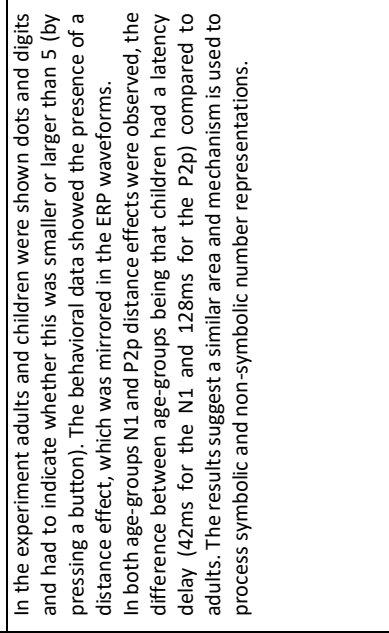 } \\
\hline 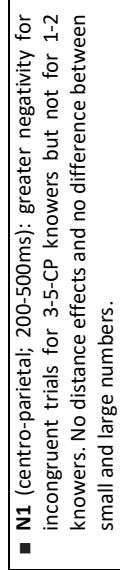 & 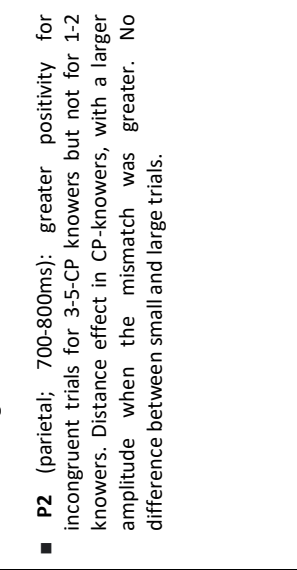 & 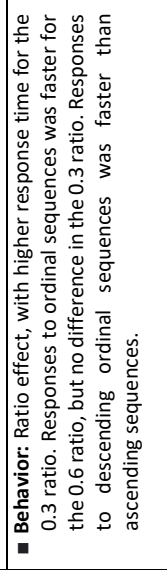 & 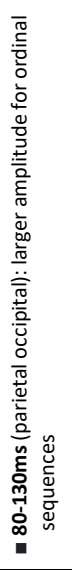 & 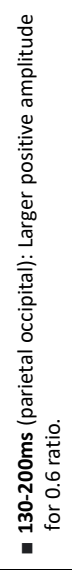 & 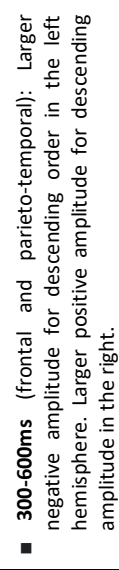 & 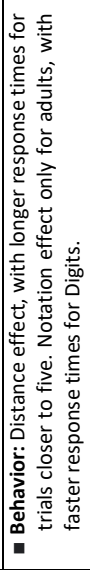 & 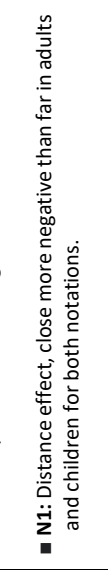 & 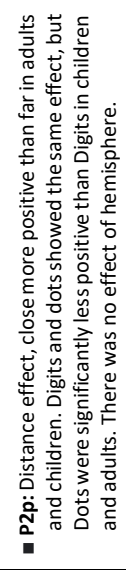 & 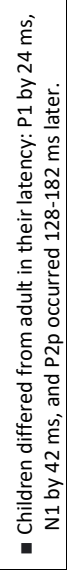 \\
\hline 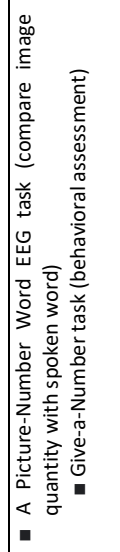 & 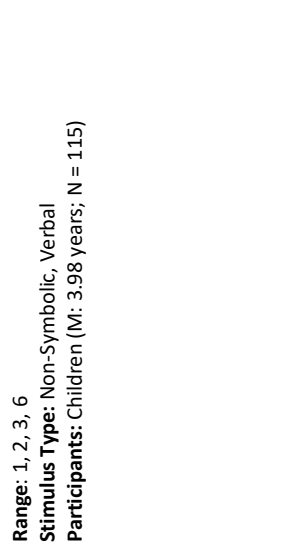 & 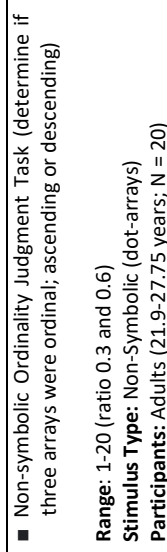 & & & & 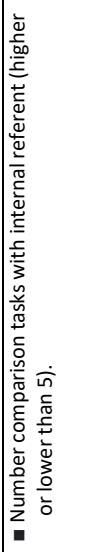 & 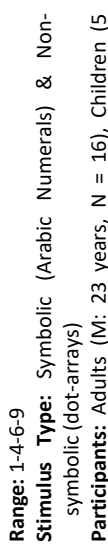 & & \\
\hline 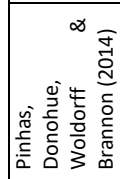 & & 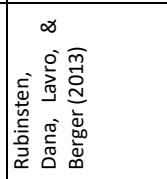 & & & & 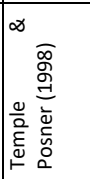 & & & \\
\hline
\end{tabular}




\begin{tabular}{|c|c|c|c|c|c|c|c|}
\hline \multicolumn{5}{|c|}{ 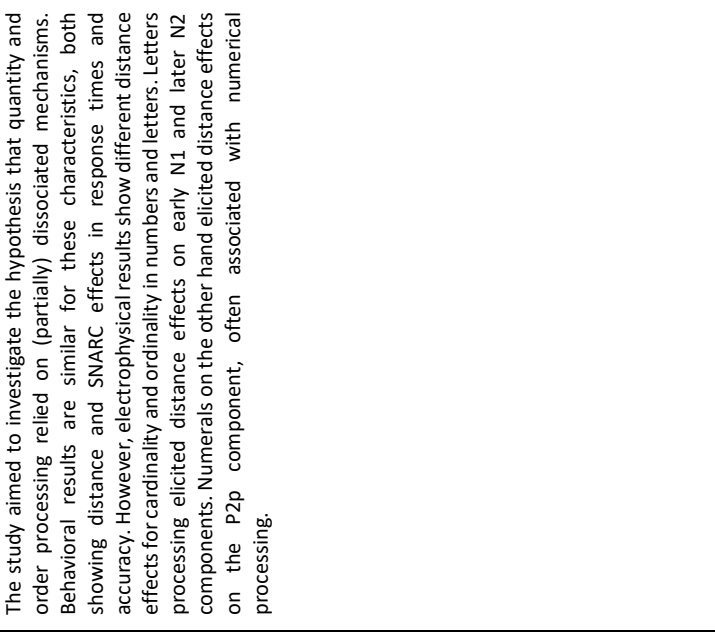 } & \multicolumn{3}{|l|}{ 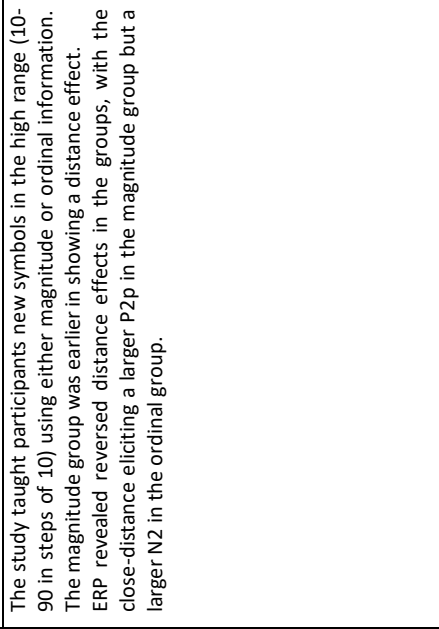 } \\
\hline 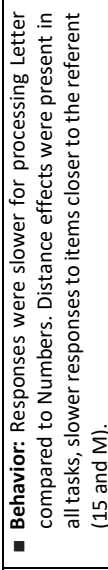 & 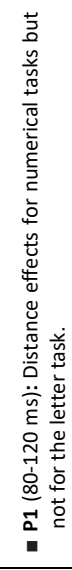 & 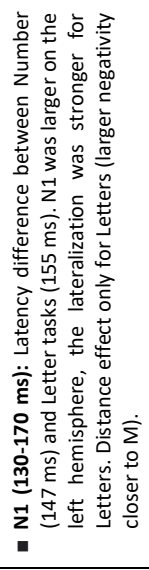 & 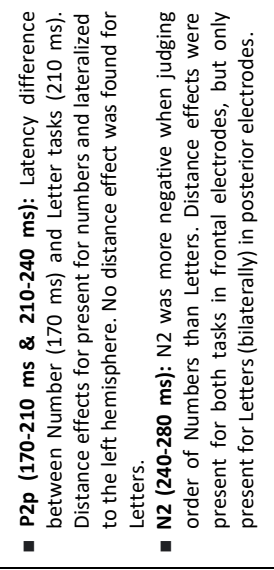 & 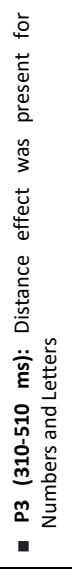 & 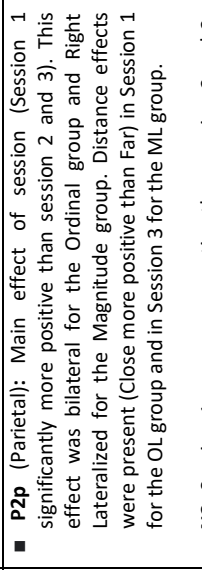 & 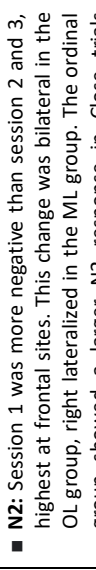 & \\
\hline \multicolumn{5}{|c|}{ 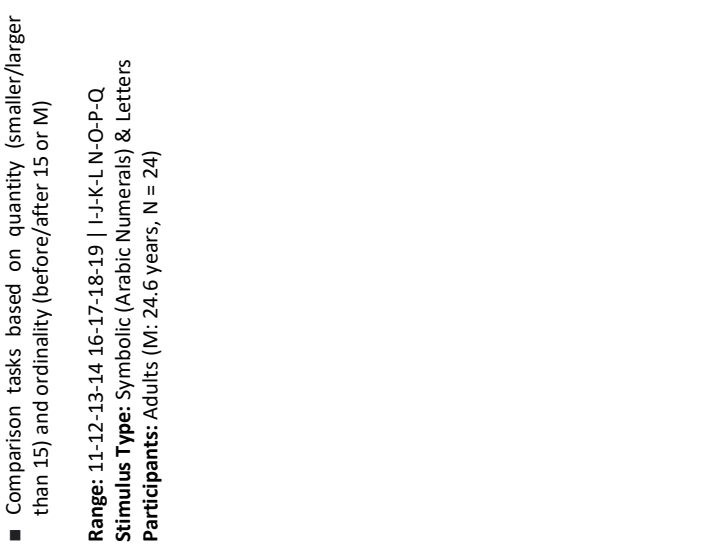 } & 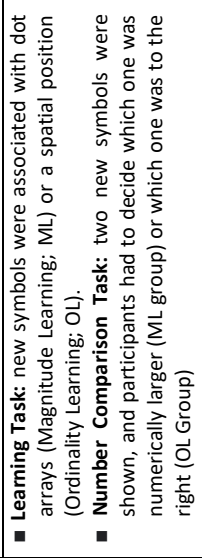 & 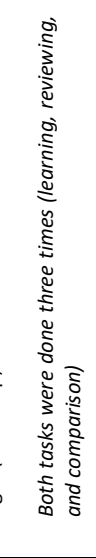 & 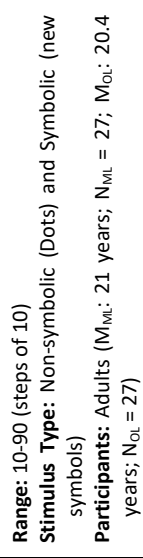 \\
\hline \multicolumn{5}{|l|}{ 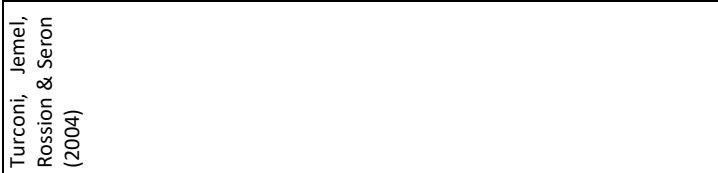 } & \multicolumn{3}{|l|}{ 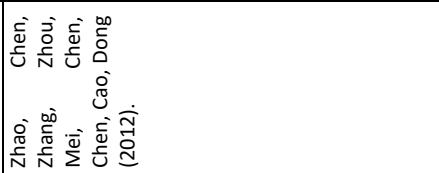 } \\
\hline
\end{tabular}


- Box 1.3

EEG measures the electrical potentials generated by large collections of neurons sending signals together. These signals make their way to the scalp, where electrodes can measure them. However, electrical signals generated by the brain are noisy and contain a mixture of many different signals sent at the same time. The noise is reduced by repeating the same types of stimuli many times (e.g., participants see a mix of 50 pictures of green lights and 50 pictures of red lights). The response to seeing the same type of stimulus (green or red light) will be similar, and we average all responses to the same types of stimuli. The averaging process extracts the signal response to a particular stimulus condition from the noise (e.g., seeing a green light vs. a red light). The averaged EEG waves are called Event-Related Potentials (ERPs) since they are all locked to the same event. These ERPs can then be compared, allowing us to investigate the differences in response to the stimulus types.

The ERP waveform consists of peaks and troughs, representing different processes depending on when and where they are detected and the specific task that generated them. Processes early in the waveform usually respond to external events, such as the physical characteristics or features of a presented stimulus, like their shape or color, and are accordingly called exogenous components (such as the P1; meaning the first positivity in the waveform). Later ERP components, such as the P3, reflect the active (conscious) processing and evaluation of a stimulus and are called endogenous components.

microV

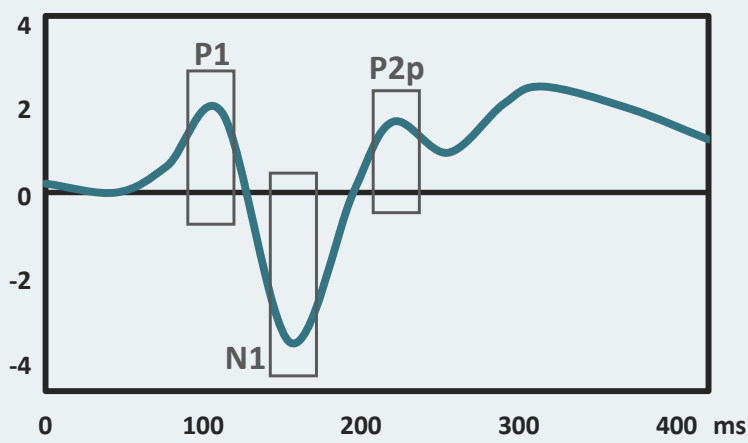

Figure B1.1: Example of an Event-Related Potential, showing the P1, N1 and P2p components 
Besides investigating potential differences in symbolic- non-symbolic mapping in the subitizing and non-subitizing ranges (Chapter 2 and Chapter 3), a second aim of this dissertation was to study the semantic representations of culture-specific finger-number patterns and how these facilitate number symbol processing. The following sections will highlight the link between the body and numerical representations, with a focus on the use of culture-specific hand gestures and the special associations they have with the numerosities they represent. Finally, studies using EPR and MEG to investigate the neural processes underlying meaningful hand gestures and finger-number representations will be discussed.

\subsection{Cultural Influences on Number Representation}

Humans display a strong connection between numbers, space, and their bodies (Hubbard et al., 2005; Wood et al., 2008). We tend to associate small numbers with the left space and larger numbers with the right (Fias et al., 1996), which also seems to be the number line's natural state seen in infants (De Hevia et al., 2014) and even newborn chicks (Rugani et al., 2008, see Box 1.2). A common finding in adults is that they are faster and more accurate when responding to small numbers with their left hand. Likewise, adults respond faster with their right hand if the number is large (also known as Spatial-Numerical Association of Response Codes, or SNARC; Dehaene and Changeux, 1993). The connection between our bodies, numbers, and space goes even further than our hands. Shaki \& Fischer (2014) made people walk around and recite random numbers whenever they made a turn. If they were reciting larger numbers, they made more right turns, and vice versa for smaller numbers and left turns. While the natural inclination for number ordering suggests a left-toright, small-to-large association between numbers, space, and our bodies; experience and culture certainly have a strong influence (e.g., right-to-left in Arabic nations; Shaki et al., 2009). This association between numbers, space, and the body aligns with the notion that cognition is rooted in bodily experiences, also known as embodied cognition (M. Wilson, 2002). 


\section{. Box 1.4}

Before the modern number system was developed, one kept track of quantities higher than four by the use of objects such as pebbles, notches in bone, knots in strings, or even sticks (known as concrete numerations). These habits can still be seen in the words used to describe numbers. The word "five" is presumably related to a proto-Indo-European word for "fist," and the Russian word for forty, "sorok" likely originates from an old Nordic word for furs ("Sekr") which were traded in bundles of forty (Wiese, 2003). As appealing as these suggestions are, it should be noted that it is extremely difficult to trace back the origins of such words since many old languages and customs have been lost to time.

One method to keep track and communicate quantities stands out, the use of body parts, and it is still in use today (Ifrah, 1985; Maor and Ifrah, 2001; Menninger, 1958). For example, tribes in Papua New Guinea have been reported to associate body-parts with different numbers, naming and touching them in a fixed order to count (Ifrah, 1985; Maor and Ifrah, 2001; Menninger, 1958). The Papuan system has words, but a word such as Doro, refers to the ring, middle, and index fingers of both hands and can mean 2, 3, 4, 19 , 20, or 21 (Maor and Ifrah, 2001). The words themselves are not numerical per se; they refer to body parts, similar to how "finger" can refer to any of our ten fingers. In order to know which of these six numbers a person is referring to when they say Doro, one has to pay attention to the gesture they are making. Is it the right-hand ring finger? Then Doro means "two". Is it the left-hand middle finger? Then Doro means "twenty" (Maor and Ifrah, 2001). The gesture determines the meaning of the number-word, on its own the word is meaningless. 


\subsubsection{The role of hands/fingers in number processing and representation}

Fischer (M. H. Fischer, 2008) demonstrated that culture-specific finger counting habits strengthen the SNARC effect (small numbers associated with left space and larger numbers with right), further extending the idea of embodiment. Left-side counters (those who started counting with their left hand) showed a stronger left-small association than rightside starters. There is a significant link between hands and numbers; both numerals and fingers can be called "digits" for a reason. Fingers provide multisensory representations of numbers; they show cardinality by the number of fingers raised and ordinality by the order in which those fingers are raised. Aside from the visual aspect of hands, humans can also differentiate fingers without visual feedback, known as finger gnosis. Humans can "feel" which fingers are touched or raised or not without seeing them. Embodiment is linked to numerical skills (Noël et al., 2005), and training finger gnosis has been shown to lead to improvements in numerical abilities (Gracia-Bafalluy and Noël, 2008). However, it is not just finger gnosis that aids us in number processing. How we arrange the fingers we raise is an important support for the representation of numerical information during simple math or calculations, so much so that even blind children (Crollen et al., 2011) and children with amputated hands/fingers (Poeck, 1964) use their (phantom-) fingers to represent numbers.

The connection between hands/fingers and numbers appears to be still present in adulthood. Although adults rarely use their hands for math, the brain areas that control our fingers were activated when adults saw a number word such as "THREE" (Tschentscher et al., 2012). Furthermore, adults returned to using their hands when asked to count the syllables in a sentence while reading it aloud, which prohibited verbal counting (Lucidi and Thevenot, 2014). A return to using hands for counting also occurred when asked about their daily experience with quantities in situations designed so they could not use number words (Pika et al., 2009). Further, Crollen et al. (2011) compared the performance of sighted, early blind, and late blind individuals on an enumeration task (e.g., name 7 tools) and on an ordered series manipulation task (e.g., how many letters between 'c' and ' $h$ '?). Verbal enumeration performance was compared between three conditions; $a$ control condition without any constraints, a hand condition where they needed to squeeze a ball, and a foot condition where they pressed a ball with their feet. The sighted and late-blind individuals used their hands when performing the counting tasks and experienced interference when their hands were occupied. The early-blind individuals did not use their hands and were also not impaired by the ball-squeezing. These results indicate the importance of sight for the early associations between hands and numbers and the presence 
of numerical embodiment in adults. Together these studies indicate that finger-number representations are embodied and still present in adults. The visual and proprioceptive feedback of fingers supports numerical processes, especially in cases where number words cannot be used, or memory capacity is limited.

Multiple behavioral studies have provided more direct evidence for shared finger and other symbolic representations of numbers in adults. An important aspect of finger-number representation is the arrangement of fingers in a fixed pattern to communicate a number (as in ordering a couple of beers). Interestingly, different cultures have different ways of communicating numbers using their hands. We learn these cultural-specific, canonical, finger-number patterns from our parents and peers, using them repeatedly until it feels strange to use any other way to show quantities. Di Luca et al. (2008) investigated the semantic association between these canonical finger-configurations and number-words by having participants name the number represented by canonical and non-canonical finger-patterns as fast as possible,. They found that the canonical finger-configurations that corresponded to the culturally learned pattern were named faster and more accurate than their unfamiliar non-canonical counterparts, despite both having the same number of fingers raised. These data provide evidence for associations between canonical finger patterns - and other symbolic number representations. Di Luca et al. (2008) speculated that the faster enumeration of canonical finger-number patterns was caused by their storage in long-term memory. Multiple authors have since added evidence in favor of this hypothesis, claiming that these finger-patterns were practiced so often that the semantic connections were strengthened to the point of automation (Andres et al., 2008; Della Sala and Anderson, 2012; Di Luca et al., 2006; Di Luca et al., 2010; Di Luca and Mauro, 2011; Di Luca and Pesenti, 2008; Soylu et al., 2019). Once automated, the hand sign's visual shape is seemingly enough to activate a numerical concept without the need to subitize or count the fingers.

This hypothesis of canonical finger-patterns being stored in long-term memory, allowing for fast and direct access to the numerosity information, was further tested in a later study by Di Luca et al. (2010). In this study, participants were primed by showing them pictures of canonical or non-canonical finger-number configurations at such short presentation rates that they could not be consciously perceived. After the finger primes, they were shown Arabic digits (the targets) that had to be named as quickly as possible. If the Arabic numeral was numerically closer to the prime (e.g., finger prime indicated number 5 and the Arabic numeral number 4), participants were faster to name the digit 
than if finger prime and digit indicated numbers further apart on the mental number line. This priming effect is explained by more representational overlap between numbers closer on the number line (see section 1.4), causing easier access to close-by numbers that were already activated by the prime. With increasing numerical distance, the priming effect decreased. The distance effect was present for canonical finger-number representations but only partially for non-canonical representations. The authors concluded that Canonical finger-numbers had a direct association with the mental number line, resulting in a distance effect similar to that found in tasks with Arabic numerals. Non-canonical finger-numbers, on the other hand, primed all numerosities up to the one they represented, leading to only partial distance effects (see Figure 1.9).

This study shows that canonical finger-number configurations are represented differently than non-canonical finger configurations and are mapped in a one-to-one fashion to other representations of number on the mental number line (in this case, Arabic digits), allowing for faster and accurate access to their numerical meaning, without the need for counting strategies. The facilitating effect of canonical finger-number configurations even extends beyond the processing of numerals. Badets et al. (2010) presented participants with simple sum-problems (e.g., $3+4)$, followed by a solution they had to verify as correct or incorrect. Solutions shown as canonical finger-number configurations were verified faster and more accurately than the same solutions presented as a series of rods. 

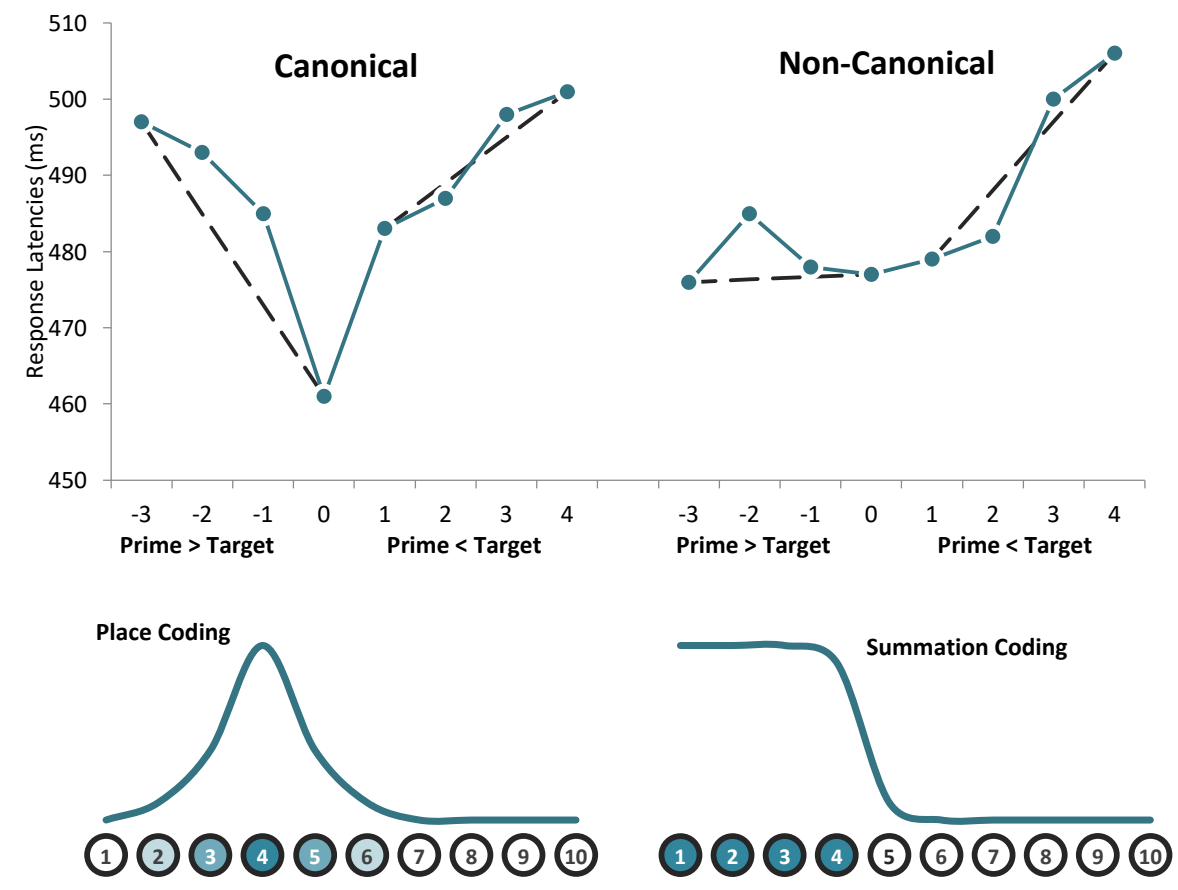

Figure 1.9: Results adapted from Di Luca et al. (2010; top) and a representation of Place and Summation coding (bottom). Distance Effects for Canonical finger-number representations were present on both sides, while Non-Canonical representations only show Distance Effects if the Target was larger than the Prime. This was speculated to be due to Place Coding (bottom left) in the canonical trials priming only a single numerosity and Summation coding (bottom right) in the Non-Canonical trials priming all numerosities up to the displayed prime.

The above-reviewed studies show that familiar finger-number gestures are a convenient way to communicate quantities and facilitate number-processing and arithmetic skills in adults. This special status of canonical finger-numbers, being able to represent numbers as a set of raised fingers in a learned canonical pattern while at the same time retaining one-to-one correspondence to the quantity they represent, puts them in an ideal position to bridge non-symbolic and symbolic number representations. Finger-numbers might thus mediate the symbol-quantity mapping process, thereby providing a base and an aid for the still-developing memory processes and counting strategies of children (Geary, 2004; Gelman and Gallistel, 1978; Soylu et al., 2018). Unfortunately, there are some in math education research who still discourage the use of external aids, such as hands, when children start learning basic mathematics with number symbols. However, cognitive research argues that such aids might help them learn these basic math skills better (see Moeller et al., 2011 for a review on this topic). The aim of the 
finger-number processing studies presented in Chapters 4 and 5, performed in adults, is to further investigate the facilitating role of (canonical) finger-number representations on number symbol processing in adults. The above-mentioned behavioral studies propose that canonical finger-numbers have stronger semantic associations with their numerical meaning. Chapter 4 and 5 look into these claims by investigating ERP components that reflect such semantic processing. These studies are intended as a first step towards investigating finger-numbers and the possible facilitative effects when learning number symbols. If finger-numbers do have such special semantic associations in adults, then this might indicate that canonical finger-number representations could be important in early number symbol acquisition, but this would need to be investigated in future studies.

In summary, prior research has shown that due to development and experience, symbolic number representations become closely connected to space and our bodies; that is, numerical cognition appears to be embodied. This embodiment is shown, for example, by a preference for the left side when reciting small numbers and a right-sided preference when reciting large numbers. The body can also affect number-processing since fullbody practice and finger-gnosis training have been shown to positively affect numerical understanding and skills (U. Fischer et al., 2011; Gracia-Bafalluy and Noël, 2008). An especially important role is reserved for our hands/fingers. We use our hands in daily life to communicate quantities using learned canonical finger-number gestures. There is some evidence from behavioral studies that such canonical finger-number configurations can facilitate the processing of number symbols or math in adults. This evidence suggests that these canonical finger-numbers have become embodied (are stored in long-term memory) and have acquired connections with other representations of numbers, allowing for fast and accurate retrieval of their numerical meaning. 


\subsubsection{ERP Studies Investigating Finger-Number Processing}

This thesis project also includes a secondary aim, that of the possible facilitation effect finger-number representations might have on the processing of number symbols. In the preceding section, behavioral data from prior studies were reviewed, suggesting that canonical finger-number representations hold a special status in long-term memory. This special status allows for fast and accurate retrieval of the finger pattern's numerical information, but few studies have investigated these effects using neural measures. One such study by Nakamura et al. (2004) using Magnetoencephalography (MEG) presented participants with meaningless and meaningful hand gestures (such as an "ok" sign). The data in that study indicated that the processing of learned, culturally meaningful signs occurs between $200-380 \mathrm{~ms}$ in the right-hemispheric inferior occipitotemporal, inferior parietal, and superior temporal sulcus.

Furthermore, two recent ERP studies investigated numerical finger-number representations. The first is a study by Proverbio \& Carminati (2019), who had participants verify the solution to simple math problems. The solution to these math problems was shown in the form of Arabic numerals (ranging between 0 and 10), but task-irrelevant canonical finger-number representations flanked the numerals. The to-be-ignored finger-numbers showed a congruent (the same number) or an incongruent (a different number) quantity. Participants were less accurate at verifying the correct or incorrect solution when a congruent finger-number representation flanked it. However, the ERP analysis in their experiment did not focus on the same location or time-window as detected by Nakamura et al. (2004). Instead, they focused on more frontal areas and possible congruency effects, which they found in the form of a late error-related negativity (750-900ms). Based on the results, they concluded that the finger-numbers were processed automatically, but this conclusion is limited because they only included canonical finger-number representations. A second study by Soylu et al. (2019) did include canonical and non-canonical finger-number representations. They measured ERPs in response to finger-numbers in the 1-4 range, showing finger-counting patterns, canonical finger montring patterns, and non-canonical finger montring patterns. Participants were shown the canonical finger pattern, followed by an Arabic numeral, and determined whether the stimuli showed the same or different quantities. Because of this comparison, participants had to process the finger patterns actively, extract a quantity, and hold this in memory. As expected, based on previous behavioral studies, canonical representations resulted in faster and more accurate responses compared to the non-canonical 
configurations. Larger amplitudes in the P1, N1, and P3 ERP components accompanied these behavioral effects. The authors interpreted the former two as evidence that canonical finger-numbers elicit more early attentional processes, whereas the latter P3 component (in a 250-500ms window) was seen as evidence for enhanced memory retrieval. However, these conclusions were limited to the subitizing range (1-4). If hands do scaffold the acquisition of number symbols, a distinction between the small subitizing range (1-4) and the larger estimation range (5-9) must be made. The current alternative theories to the ANS Mapping account emphasize a difference between how these ranges are acquired (see section 1.3).

In summary, research including neural measures of finger-number representations is as yet very scarce. Studies done on non-numerical hand meaning (Avikainen et al., 2003; Nakamura et al., 2004) point towards an occipital and parietal network responsible for determining a hand sign's meaning, showing activation 200-380ms after seeing the stimulus. Studies using numerical hand patterns (Proverbio and Carminati, 2019; Soylu et al., 2019) show that canonical finger-number representations elicit higher activation in these areas when compared to non-canonical representations, alluding to more attentional and memory resources being used when processing culturally learned canonical finger configurations. 


\subsection{Outline of this Thesis}

The current dissertation aims to add to the literature discussed in the previous sections by investigating how numerical symbols (such as Arabic numerals) are represented and processed in the brain. The first two studies described in Chapter $\mathbf{2}$ and $\mathbf{3}$ tested the assumptions of the ANS mapping account. The ANS mapping account assumes that all symbols gain meaning by mapping onto non-symbolic representations (as discussed in Section 1.3.1). Although the ANS Mapping account by Dehaene (Dehaene, 2001) has been dominant for many years, recent reviews and studies dispute its assumptions (see Section 1.3.2). The Exact Algorithms Hypothesis (Carey, 2009; Carey and Barner, 2019) proposes that direct one-to-one mapping is limited to the subitizing range and suggests that acquisition of number symbols outside the subitizing range takes place via symbol-tosymbol mapping. Suppose the ANS mapping account is correct in assuming all symbols map onto the non-symbolic ANS estimates. In that case, there should be no difference between learning symbols in the subitizing and non-subitizing ranges. However, based on the Exact Algorithms Hypothesis (Carey and Barner, 2019), one would expect only the subitizing range to gain numerical meaning by mapping onto non-symbolic representations.

The studies described in Chapter $\mathbf{4}$ and $\mathbf{5}$ aimed to investigate the semantic processing and possible facilitating effect of culture-specific (canonical) finger-number representations in adults. As discussed in Section 1.5, numbers are thought to be embodied due to the intuitive use of hands when communication quantities to others. Previous research proposed that canonical finger-number configurations have strong associations with their numerical meaning (Andres et al., 2008; Di Luca et al., 2010; Di Luca and Mauro, 2011; Di Luca and Pesenti, 2008). The stronger associations of canonical finger-number patterns might mediate number symbol learning, scaffolding the mapping process by providing an external memory aid. The repeated use of hands to aid in number processing would lead to an embodiment of canonical finger-number representations, still visible in adults. Studies using neural measures to confirm this conclusion are scarce, with only two experiments including EEG measures (see Proverbio and Carminati, 2019 and Soylu et al., 2019 in section 1.4). However, these experiments did not compare canonical and non-canonical finger-number representations in the subitizing and non-subitizing range. A distinction between these ranges is important; as discussed in section 1.3.2, one-to-one mapping might only occur for the first four symbols. Suppose mapping is limited to the first four symbols. In that case, we expect a stronger association between finger-numbers and other numerical representations in that 1-4 range since finger-numbers would not have mediated the acquisition of larger numbers 
The question addressed in the study described in Chapter $\mathbf{2}$ is whether number symbols 1-9 gain numerical meaning through direct mapping onto non-symbolic representations of magnitude or numerical size. We investigate this question using a trial-and-error learning task in which adults have to learn to assign numerical meaning to novel symbols by associating/mapping them onto non-symbolic stimuli. While several studies reviewed in this introduction employ neural measures to investigate number processing, there is a striking gap in the literature regarding changes in neural correlates when symbols gain meaning. Hence, the learning processes by which symbols gain numeric meaning are as good as unexplored (see Section 1.4, Table 1). The investigation of the subitizing and non-subitizing ranges allows us to test the assumptions of the aforementioned ANS mapping account and the Exact Algorithms Hypothesis. Based on the evidence discussed in section 1.3 we expect symbols in the subitizing range to gain an exact meaning since it is possible to extract an exact quantity from the non-symbolic stimuli to map a symbol onto. The acquisition of symbols in this range would be accompanied by performance improvements (faster response times and higher accuracy) and smaller P2p amplitudes (indicative of less cognitive effort to retrieve the numerical meaning of the symbol).

Chapter 3 investigates whether the development of symbolic and non-symbolic number processing follows the predictions of the ANS mapping account. Instead of a learning task, as was the case in Chapter 2, we compare longitudinal data from children when they were in Kindergarten ( 5 years old) and Grade 1 (6 years old) to data from adults (22 years old). Both groups performed a similar number comparison task, in which they compared Symbolic (Arabic Numeral) and Non-Symbolic stimuli (Dot-Arrays) to an internal reference of five. The children in this age group are expected to have already gained the cardinality principles. However, as predicted by the Exact Algorithms Hypothesis, they would rely on symbol-to-symbol associations and algorithms to identify quantities larger than four (see Section 1.3.2). We expect children to show behavioral distance effects for the subitizing and non-subitizing ranges since both numerical and semantic relationships can elicit these distance effects. However, we expect P2p distance effects only to occur when a numerical meaning is accessed, which we expect only in the subitizing range where symbols map directly onto non-symbolic representations. 
Chapter 4 describes a study in which we look at the potential facilitatory effects of canonical (compared to non-canonical) finger-number representations on number processing in a math verification task. We presented participants with simple math problems to solve, followed by an answer in the form of canonical or non-canonical finger-number representations. They had to verify this solution as correct or incorrect, thus actively processing the numerical meaning of the hands and comparing it to a number held in memory. We expect the canonical hands to be verified faster and more accurately than non-canonical hands, which is in line with previous literature. To investigate the hypothesis that these canonical patterns have stronger associations with their numerical meaning stored in long-term memory, we look at ERP components associated with early semantic process (first access to numerical meaning; the P2p component) and memory recognition and stimulus categorization processes (the $\mathrm{P} 3$ component). We expect these components to show a larger response to canonical finger-number patterns, indicating memory processes rather than counting processes. In line with the previous chapters and the Exact Algorithm Hypothesis, we also expect these associations between canonical finger-number representations and their numerical meaning to be stronger in the subitizing range, where a possible mediation role would be stronger.

Lastly, Chapter $\mathbf{5}$ complemented the study in Chapter $\mathbf{4}$ using a different paradigm. This study investigated the facilitatory effect of canonical finger-number representation on number processing in the small (1-4) and large (6-9) range during a match-to-sample task but now presenting numerosity congruent (same trials) or incongruent (different trials) finger-patterns and Arabic digits within one stimulus display (this in contrast to the experiment in Chapter 4 that required participants to compare a finger-number representation to a quantity held in memory). Participants had to decide if the numerals and hands showed the same or different quantities. In contrast to Chapter 4, the two representations now directly affect each other, enhancing the possible facilitation effect finger-numbers might have on the processing of Arabic numerals. We expect only the canonical finger-number patterns to facilitate the processing (and comparison) of matching Arabic numerals for the same reasons discussed in the previous paragraph. And like in the previous chapters, we expect these effects to be stronger in the subitizing range. 


\subsection{References}

Agrillo, C., Dadda, M., Serena, G., \& Bisazza, A. (2008). Do fish count? spontaneous discrimination of quantity in female mosquitofish. Animal Cognition, 11(3), 495-503. https://doi.org/10.1007/s10071-008-0140-9 (cited on page 12)

Andres, M., Di Luca, S., \& Pesenti, M. (2008). Open peer commentary: Finger counting: The missing tool? Behavioral and Brain Sciences, 31(6), 642-643. https://doi. org/10.1017/S0140525X08005578 (cited on pages 10, 44, 50)

Ansari, D., Garcia, N., Lucas, E., Hamon, K., \& Dhitalm, B. (2005). Neural correlates of symbolic number processing in children and adults. Neuroreport, 16(16), 1769-1773. https://doi.org/10.1097/01.wnr.0000183905.23396.f1 (cited on page 30)

Avikainen, S., Liuhanen, S., Schürmann, M., \& Hari, R. (2003). Enhanced extrastriate activation during observation of distorted finger postures. Journal of Cognitive Neuroscience, 15(5), 658-663. https://doi.org/10.1162/jocn.2003.15.5.658 (cited on page 49)

Badets, A., Pesenti, M., \& Olivier, E. (2010). Response-effect compatibility of fingernumeral configurations in arithmetical context. Quarterly Journal of Experimental Psychology, 63(1), 16-22. https://doi.org/10.1080/17470210903134385 (cited on page 45)

Barth, H., Kanwisher, N., \& Spelke, E. (2003). The construction of large number representations in adults. Cognition, 86(3), 201-221. https://doi.org/10.1016/S0010-0277(02)00178-6 (cited on page 19)

Bonanni, R., Natoli, E., Cafazzo, S., \& Valsecchi, P. (2011). Free-ranging dogs assess the quantity of opponents in intergroup conflicts. Animal Cognition, 14(1), 103-115. https://doi.org/10.1007/s10071-010-0348-3 (cited on page 12)

Buckley, P. B., \& Gillman, C. B. (1974). Comparisons of digits and dot patterns. Journal of experimental psychology, 103(6), 1131-1136. https://doi.org/10.1037/h0037361 (cited on page 18)

Cai, D., Zhang, L., Li, Y., Wei, W., \& Georgiou, G. K. (2018). The role of approximate number system in different mathematics skills across grades. Frontiers in Psychology, 9(SEP), 1733. https://doi.org/10.3389/fpsyg.2018.01733 (cited on page 10)

Cantlon, J. F., \& Brannon, E. M. (2006). Shared system for ordering small and large numbers in monkeys and humans. Psychological Science, 17(5), 401-406. https: //doi.org/10.1111/j.1467-9280.2006.01719.x (cited on page 25) 
Carey, S. (2009). The origin of concepts (Volume 133). Oxford University Press. https: //doi.org/10.1093/acprof:oso/9780195367638.001.0001. (Cited on pages 22, 23, $25,28,50)$

Carey, S., \& Barner, D. (2019). Ontogenetic origins of human integer representations. Trends in Cognitive Sciences, 23(10), 823-835. https://doi.org/10.1016/j.tics. 2019.07.004 (cited on pages 21-23, 28, 29, 50)

Chiao, J. Y., Bordeaux, A. R., \& Ambady, N. (2004). Mental representations of social status. Cognition, 93(2). https://doi.org/10.1016/j.cognition.2003.07.008 (cited on page 26)

Condry, K. F., \& Spelke, E. S. (2008). The development of language and abstract concepts: The case of natural number. Journal of Experimental Psychology: General, 137(1), 22-38. https://doi.org/10.1037/0096-3445.137.1.22 (cited on page 21)

Cowan, N. (2001). The magical number 4 in short-term memory: A reconsideration of mental storage capacity. Behavioral and Brain Sciences, 24(1), 87-114. https: //doi.org/10.1017/S0140525X01003922 (cited on page 15)

Crollen, V., Mahe, R., Collignon, O., \& Seron, X. (2011). The role of vision in the development of finger-number interactions: Finger-counting and finger-montring in blind children. Journal of Experimental Child Psychology, 109(4), 525-539. https://doi.org/10.1016/j.jecp.2011.03.011 (cited on page 43)

De Hevia, M. D., Girelli, L., Addabbo, M., \& Cassia, V. M. (2014). Human infants' preference for left-to-right oriented increasing numerical sequences. PLOS ONE, 9(5). https://doi.org/10.1371/journal.pone.0096412 (cited on pages 24, 41)

Dehaene, S. (1996). The organization of brain activations in number comparison: Event-related potentials and the additive-factors method. Journal of Cognitive Neuroscience, 8(1), 47-68. https://doi.org/10.1162/jocn.1996.8.1.47 (cited on pages 31,32 )

Dehaene, S. (2001, February). Précis of the number sense. Mind \& language (Pages 16-36). https://doi.org/10.1111/1468-0017.00154. (Cited on pages 18, 25, 28, 50)

Dehaene, S. (2003). The neural basis of the weber-fechner law: A logarithmic mental number line. Trends in Cognitive Sciences, 7(4), 145-147. https://doi.org/10. 1016/S1364-6613(03)00055-X (cited on page 18)

Dehaene, S. (2007). Symbols and quantities in parietal cortex: Elements of a mathematical theory of number representation and manipulation. Attention \& performance xxii. sensori-motor ... (Pages 527-574). https :// doi .org/10.1093/acprof: oso/9780199231447.003.0024. (Cited on page 18) 
Dehaene, S. (2011). The number sense. how the mind creates mathematics, revised and updated edition. Oxford University Press. https://doi.org/10.2307/2589308. (Cited on pages 9, 13, 18, 22, 25)

Dehaene, S., \& Brannon, E. (2011). Space, time and number in the brain searching for the foundations of mathematical thought. Elsevier Academic Press. (Cited on page 22).

Dehaene, S., \& Changeux, J.-P. P. (1993). Development of elementary numerical abilities: A neuronal model. Journal of Cognitive Neuroscience, 5(4), 390-407. https: //doi.org/10.1162/jocn.1993.5.4.390 (cited on pages 18, 41)

Dehaene, S., Dehaene-Lambertz, G., \& Cohen, L. (1998). Abstract representations of numbers in the animal and human brain. Trends in Neurosciences, 21(8), 355-361. https://doi.org/10.1016/S0166-2236(98)01263-6 (cited on pages 17, 18)

Dehaene, S., Izard, V., Spelke, E., \& Pica, P. (2008). Log or linear? distinct intuitions of the number scale in western and amazonian indigene cultures. Science (New York, N.Y.), 320(5880), 1217-20. https://doi.org/10.1126/science.1156540 (cited on page 18)

Della Sala, S., \& Anderson, M. (2012). Neuroscience in education: The good, the bad, and the ugly. Oxford University Press. https://doi.org/10.1093/acprof:oso/ 9780199600496.001.0001. (Cited on page 44)

Di Luca, S., Granà, A., Pesenti, M., Semenza, C., \& Seron, X. (2006). Finger-digit compatibility in arabic numeral processing. Quarterly Journal of Experimental Psychology, 59(9), 1648-1663. https://doi.org/10.1080/17470210500256839 (cited on page 44)

Di Luca, S., Lefèvre, N., \& Pesenti, M. (2010). Place and summation coding for canonical and non-canonical finger numeral representations. Cognition, 117(1), 95-100. https://doi.org/10.1016/j.cognition.2010.06.008 (cited on pages 44, 50)

Di Luca, S., \& Mauro, P. (2011). Finger numeral representations: More than just another symbolic code. Frontiers in Psychology, 2(NOV), 272. https://doi.org/10.3389/ fpsyg.2011.00272 (cited on pages 10, 44, 50)

Di Luca, S., \& Pesenti, M. (2008). Masked priming effect with canonical finger numeral configurations. Experimental Brain Research, 185(1), 27-39. https://doi.org/10. 1007/s00221-007-1132-8 (cited on pages 44, 50)

Diester, I., \& Nieder, A. (2007). Semantic associations between signs and numerical categories in the prefrontal cortex (S. Dehaene, Editor). PLoS Biology, 5(11), 2684-2695. https://doi.org/10.1371/journal.pbio.0050294 (cited on pages 30, 34) 
Diester, I., \& Nieder, A. (2010). Numerical values leave a semantic imprint on associated signs in monkeys. Journal of cognitive neuroscience, 22(1), 174-83. https://doi. org/10.1162/jocn.2009.21193 (cited on pages 30, 34)

Duncan, G. J., Dowsett, C. J., Claessens, A., Magnuson, K., Huston, A. C., Klebanov, P., Pagani, L. S., Feinstein, L., Engel, M., Brooks-Gunn, J., Sexton, H., Duckworth, K., \& Japel, C. (2007). School readiness and later achievement. Developmental Psychology, 43(6), 1428-1446. https://doi.org/10.1037/0012-1649.43.6.1428 (cited on page 10)

Elliott, L., Feigenson, L., Halberda, J., \& Libertus, M. E. (2019). Bidirectional, longitudinal associations between math ability and approximate number system precision in childhood. Journal of Cognition and Development, 20(1), 56-74. https://doi.org/ 10.1080/15248372.2018.1551218 (cited on page 10)

Feigenson, L., \& Carey, S. (2005). On the limits of infants' quantification of small object arrays. Cognition, 97(3), 295-313. https://doi.org/10.1016/j.cognition.2004.09. 010 (cited on page 16)

Feigenson, L., Carey, S., \& Hauser, M. (2002). The representations underlying infants' choice of more: Object files versus analog magnitudes. Psychological Science, 13(2), 150-156. https://doi.org/10.1111/1467-9280.00427 (cited on page 16)

Feigenson, L., Dehaene, S., \& Spelke, E. (2004). Core systems of number. Trends in Cognitive Sciences, 8(7), 307-314. https://doi.org/10.1016/j.tics.2004.05.002 (cited on pages $9,13,15,18$ )

Fias, W., Brysbaert, M., Geypens, F., \& d'Ydewalle, G. (1996). The importance of magnitude information in numerical processing: Evidence from the snarc effect. Mathematical Cognition, 2(1), 95-110. https://doi.org/10.1080/135467996387552 (cited on page 41)

Fischer, M. H. (2008). Finger counting habits modulate spatial-numerical associations. Cortex, 44(4), 386-392. https://doi.org/10.1016/j.cortex.2007.08.004 (cited on page 43)

Fischer, U., Moeller, K., Bientzle, M., Cress, U., \& Nuerk, H. C. (2011). Sensori-motor spatial training of number magnitude representation. Psychonomic Bulletin and Review, 18(1), 177-183. https://doi.org/10.3758/s13423-010-0031-3 (cited on page 47)

Fuson, K. C. (1988). Children's counting and concepts of number (Illustrate, Volume 31). Springer New York. https://doi.org/10.1007/978-1-4612-3754-9. (Cited on page 21) 
Fuson, K. C. (2012). Children's counting and concepts of number. Springer New York. (Cited on page 21).

Gallistel, C. R., \& Gelman, R. (2000). Non-verbal numerical cognition: From reals to integers. Trends in Cognitive Sciences, 4(2), 59-65. https://doi.org/10.1016/ S1364-6613(99)01424-2 (cited on pages 17, 18)

Geary, D. C. (2004). Mathematics and learning disabilities. Journal of Learning Disabilities, 37(1), 4-15. https://doi.org/10.1177/00222194040370010201 (cited on pages 11,46$)$

Geary, D. C. (2011). Cognitive predictors of achievement growth in mathematics: A 5-year longitudinal study. Developmental psychology, 47(6), 1539-52. https: //doi.org/10.1037/a0025510 (cited on page 10)

Gebuis, T., \& Reynvoet, B. (2013). The neural mechanisms underlying passive and active processing of numerosity. NeuroImage, 70, 301-307. https://doi .org/https : //doi.org/10.1016/j.neuroimage.2012.12.048 (cited on page 33)

Gelman, R., \& Gallistel, C. R. (1978). The child's understanding of number. Harvard University Press. (Cited on pages 11, 46).

Gouet, C., Gutiérrez Silva, C. A., Guedes, B., \& Peña, M. (2018). Cognitive and neural effects of a brief nonsymbolic approximate arithmetic training in healthy first grade children. Frontiers in integrative neuroscience, 12, 28. https://doi.org/10. 3389/fnint.2018.00028 (cited on page 31)

Gracia-Bafalluy, M., \& Noël, M. P. (2008). Does finger training increase young children's numerical performance? Cortex, 44(4), 368-375. https://doi.org/10.1016/j.cortex. 2007.08.020 (cited on pages 43, 47)

Halberda, J., Mazzocco, M. M. M., \& Feigenson, L. (2008). Individual differences in nonverbal number acuity correlate with maths achievement. Nature, 455(October), 665-668. https://doi.org/10.1038/nature07246 (cited on page 20)

Harnad, S. (1990). The symbol grounding problem. Physica D: Nonlinear Phenomena, 42(1-3), 335-346. https://doi.org/10.1016/0167-2789(90)90087-6 (cited on page 9)

Harnad, S. (2003). Symbol grounding problem. In L. Nadel (Editor), Encyclopedia of cognitive science (Pages 335-346). Nature Publishing Group: Macmillan. (Cited on page 9).

Harvey, B. M., Klein, B. P., Petridou, N., \& Dumoulin, S. O. (2013). Topographic representation of numerosity in the human parietal cortex. Science, 341(6150), 1123-1126. https://doi.org/10.1126/science.1239052 (cited on page 30) 
Hersh, R., Dehaene, S., \& Hersh, R. (1999). The number sense: How the mind creates mathematics. The American Mathematical Monthly, 105(10), 975. https://doi.org/ 10.2307/2589308 (cited on page 25)

Howard, S. R., Avarguès-Weber, A., Garcia, J. E., Greentree, A. D., \& Dyer, A. G. (2018). Numerical ordering of zero in honey bees. Science, 360(6393), 1124-1126. https: //doi.org/10.1126/science.aar4975 (cited on page 14)

Howard, S. R., Avarguès-Weber, A., Garcia, J. E., Greentree, A. D., \& Dyer, A. G. (2019). Numerical cognition in honeybees enables addition and subtraction. Science Advances, 5(2), eaav0961. https://doi.org/10.1126/sciadv.aav0961 (cited on page 14)

Hubbard, E. M., Piazza, M., Pinel, P., \& Dehaene, S. (2005). Interactions between number and space in parietal cortex. Nature Reviews Neuroscience, 6(6), 435-448. https: //doi.org/10.1038/nrn1684 (cited on page 41)

Hutchison, J. E., Ansari, D., Zheng, S., De Jesus, S., \& Lyons, I. M. (2019). The relation between subitizable symbolic and non-symbolic number processing over the kindergarten school year. Developmental Science, 00:e12884. https://doi.org/10. 1111/desc.12884 (cited on page 29)

Hyde, D. C., \& Spelke, E. S. (2009). All numbers are not equal: An electrophysiological investigation of small and large number representations. Journal of cognitive neuroscience, 21(6), 1039-1053. https://doi.org/10.1162/jocn.2009.21090 (cited on pages 31,32 )

Ifrah, G. (1985). From one to zero: A universal history of numbers. Viking Penguin. https://doi.org/10.1080/00029890.1989.11972184. (Cited on page 42)

Izard, V., Dehaene-Lambertz, G., \& Dehaene, S. (2008). Distinct cerebral pathways for object identity and number in human infants (M. Posner, Editor). PLoS Biology, 6(2), 0275-0285. https://doi.org/10.1371/journal.pbio.0060011 (cited on page 19)

Izard, V., Sann, C., Spelke, E. S., \& Streri, A. (2009). Newborn infants perceive abstract numbers. Proceedings of the National Academy of Sciences of the United States of America, 106(25), 10382-5. https://doi.org/10.1073/pnas.0812142106 (cited on page 19)

Jaakkola, K., Fellner, W., Erb, L., Rodriguez, M., \& Guarino, E. (2005). Understanding of the concept of numerically "less" by bottlenose dolphins (tursiops truncatus). Journal of Comparative Psychology, 119(3), 296-303. https://doi.org/10.1037/ 0735-7036.119.3.296 (cited on page 12) 
Kaufman, E. L., Lord, M. W., Reese, T., \& Volkmann, J. (1949). The discrimination of visual number. The American journal of psychology, 62(4), 498-525. https: //doi.org/10.2307/1418556 (cited on pages 12, 15, 18)

Kaufmann, L., Koppelstaetter, F., Siedentopf, C., Haala, I., Haberlandt, E., Zimmerhackl, L. B., Felber, S., \& Ischebeck, A. (2006). Neural correlates of the number-size interference task in children. NeuroReport, 17(6), 587-591. https://doi.org/10. 1097/00001756-200604240-00007 (cited on page 30)

Krajcsi, A., Lengyel, G., \& Kojouharova, P. (2016). The source of the symbolic numerical distance and size effects. Frontiers in Psychology, 7, 1795. https://doi.org/10. 3389/fpsyg.2016.01795 (cited on page 29)

Le Corre, M., \& Carey, S. (2007). One, two, three, four, nothing more: An investigation of the conceptual sources of the verbal counting principles. Cognition, 105(2), 395-438. https://doi.org/10.1016/j.cognition.2006.10.005 (cited on page 25)

Leibovich, T., \& Ansari, D. (2016a). The symbol-grounding problem in numerical cognition: A review of theory, evidence, and outstanding questions. Canadian Journal of Experimental Psychology, 70(1), 12-23. https://doi.org/10.1037/cep0000070 (cited on page 25)

Leibovich, T., \& Ansari, D. (2016b). The Symbol-Grounding Problem in Numerical Cognition: A Review of Theory, Evidence, and Outstanding Questions. Canadian Journal of Experimental Psychology, 70(1), 12-23. https://doi.org/10.1037/ cep0000070 (cited on page 9)

Leslie, A. M., Xu, F., Tremoulet, P. D., \& Scholl, B. J. (1998). Indexing and the object concept: Developing 'what' and 'where' systems. Trends in Cognitive Sciences, 2(1), 10-18. https://doi.org/10.1016/S1364-6613(97)01113-3 (cited on page 15)

Libertus, M. E. (2019, January 1). Understanding the link between the approximate number system and math abilities. Cognitive foundations for improving mathematical learning (Pages 91-106). Elsevier. https://doi.org/10.1016/b978-0-12-8159521.00004-9. (Cited on page 10)

Libertus, M. E., \& Brannon, E. M. (2010). Stable individual differences in number discrimination in infancy. Developmental Science, 13(6), 900-906. https://doi.org/10.1111/j.1467-7687.2009.00948.x (cited on pages 20, 25)

Libertus, M. E., Woldorff, M. G., \& Brannon, E. M. (2007). Electrophysiological evidence for notation independence in numerical processing. (Volume 3). https://doi.org/ 10.1186/1744-9081-3-1. (Cited on pages 31, 32) 
Lucidi, A., \& Thevenot, C. (2014). Do not count on me to imagine how i act: Behavior contradicts questionnaire responses in the assessment of finger counting habits. Annales des Telecommunications/Annals of Telecommunications, 46(4), 1079-1087. https://doi.org/10.3758/s13428-014-0447-1 (cited on page 43)

Lyons, I. M., Ansari, D., \& Beilock, S. L. (2012). Symbolic estrangement: Evidence against a strong association between numerical symbols and the quantities they represent. Journal of experimental psychology. General, 141(4), 635-41. https: //doi.org/10.1037/a0027248 (cited on pages 27, 28)

Mandler, G., \& Shebo, B. J. (1982). Subitizing: An analysis of its component processes. Journal of Experimental Psychology: General, 111(1), 1-22. https://doi.org/10. 1037/0096-3445.111.1.1 (cited on page 15)

Maor, E., \& Ifrah, G. (2001). The universal history of numbers: From prehistory to the invention of the computer (Volume 108). Wiley. https://doi.org/10.2307/2695263. (Cited on page 42)

Marinova, M., Sasanguie, D., \& Reynvoet, B. (2021). Numerals do not need numerosities: Robust evidence for distinct numerical representations for symbolic and nonsymbolic numbers. Psychological Research, 85(2), 1-13. https://doi.org/10.1007/ s00426-019-01286-z (cited on page 27)

Matthews, P., \& Chesney, D. (2011). Straightening up: Number line estimates shift from $\log$ to linear with additional information. Proceedings of the 33rd ..., 1936-1941 (cited on page 23).

McComb, K., Packer, C., \& Pusey, A. (1994). Roaring and numerical assessment in contests between groups of female lions, panthera leo. Animal Behaviour, 47(2), 379-387. https://doi.org/10.1006/anbe.1994.1052 (cited on pages 12, 14)

Menninger, K. (1958). Zahlwort und ziffer: Eine kulturgeschichte der zahl (2nd edition). Vandenhoeck \& Ruprecht. (Cited on page 42).

Merkley, R., Shimi, A., \& Scerif, G. (2016). Electrophysiological markers of newly acquired symbolic numerical representations: The role of magnitude and ordinal information. ZDM - Mathematics Education, 48(3), 279-289. https://doi.org/10. 1007/s11858-015-0751-y (cited on pages 33, 34)

Moeller, K., Martignon, L., Wessolowski, S., Engel, J., \& Nuerk, H. C. (2011). Effects of finger counting on numerical development the opposing views of neurocognition and mathematics education. Frontiers in Psychology, 2(NOV), 328. https://doi. org/10.3389/fpsyg.2011.00328 (cited on page 46) 
Moyer, R. S., \& Landauer, T. K. (1967). Time required for judgements of numerical inequality. Nature, 215(5109), 1519-1520. https://doi.org/10.1038/2151519a0 (cited on page 25)

Nakamura, A., Maess, B., Knösche, T. R., Gunter, T. C., Bach, P., \& Friederici, A. D. (2004). Cooperation of different neuronal systems during hand sign recognition. NeuroImage, 23(1), 25-34. https://doi.org/10.1016/j.neuroimage.2004.04.034 (cited on pages 48,49 )

Nieder, A. (2020). Neural constraints on human number concepts. Current Opinion in Neurobiology, 60, 28-36. https://doi.org/10.1016/j.conb.2019.10.003 (cited on page 27)

Noël, M. P., Gracia-Bafalluy, M., \& Noël, M. P. (2005). Finger gnosia: A predictor of numerical abilities in children? Cortex, 11(5), 413-430. https://doi.org/10.1080/ 09297040590951550 (cited on page 43)

Piazza, M. (2010). Neurocognitive start-up tools for symbolic number representations. Trends in Cognitive Science, 14(12), 542-551. https://doi.org/10.1016/j.tics.2010. 09.008 (cited on pages $9,15,20,25$ )

Piazza, M., Facoetti, A., Trussardi, A. N., Berteletti, I., Conte, S., Lucangeli, D., Dehaene, S., \& Zorzi, M. (2010). Developmental trajectory of number acuity reveals a severe impairment in developmental dyscalculia. Cognition, 116(1), 33-41. https: //doi.org/10.1016/j.cognition.2010.03.012 (cited on page 20)

Pika, S., Nicoladis, E., \& Marentette, P. (2009). How to order a beer: Cultural differences in the use of conventional gestures for numbers. Journal of Cross-Cultural Psychology, 40(1), 70-80. https://doi.org/10.1177/0022022108326197 (cited on page 43)

Pinel, P., Dehaene, S., Rivière, D., \& LeBihan, D. (2001). Modulation of parietal activation by semantic distance in a number comparison task. NeuroImage, 14(5), 1013-1026. https://doi.org/10.1006/nimg.2001.0913 (cited on pages 31, 32)

Plotnik, J. M., Brubaker, D. L., Dale, R., Tiller, L. N., Mumby, H. S., \& Clayton, N. S. (2019). Elephants have a nose for quantity. Proceedings of the National Academy of Sciences of the United States of America, 116(25), 12566-12571. https://doi. org/10.1073/pnas.1818284116 (cited on page 12)

Poeck, K. (1964). Phantoms following amputation in early childhood and in congenital absence of limbs. Cortex, 1(3), 269-275. https :// doi .org/10.1016/s0010 9452(64)80002-2 (cited on page 43) 
Proverbio, A. M., \& Carminati, M. (2019). Finger-counting observation interferes with number processing. Neuropsychologia, 131, 275-284. https://doi.org/10.1016/j. neuropsychologia.2019.06.001 (cited on pages 48-50)

Pylyshyn, Z. W. (1994). Some primitive mechanisms of spatial attention. Cognition, 50(13), 363-384. https://doi.org/10.1016/0010-0277(94)90036-1 (cited on page 15)

Pylyshyn, Z. W., \& Storm, R. W. (1988). Tracking multiple independent targets: Evidence for a parallel tracking mechanism. Spatial vision, 3(3), 179-197. https://doi.org/ 10.1163/156856888X00122 (cited on page 15)

Ramani, G. B., \& Siegler, R. S. (2008). Promoting broad and stable improvements in lowincome children's numerical knowledge through playing number board games. Child Development, 79(2), 375-394. https://doi.org/10.1111/j.1467-8624.2007. 01131.x (cited on page 23)

Range, F., Jenikejew, J., Schröder, I., \& Virányi, Z. (2014). Difference in quantity discrimination in dogs and wolves. Frontiers in Psychology, 5(NOV), 1299. https://doi.org/10.3389/fpsyg.2014.01299 (cited on page 12)

Reynvoet, B., \& Sasanguie, D. (2016). The symbol grounding problem revisited: A thorough evaluation of the ans mapping account and the proposal of an alternative account based on symbol-symbol associations. Frontiers in Psychology, 7(OCT), 1581. https://doi.org/10.3389/fpsyg.2016.01581 (cited on pages 25, 26)

Reznikova, Z., Panteleeva, S., \& Vorobyeva, N. (2019). Precise relative-quantity judgement in the striped field mouse apodemus agrarius pallas. Animal Cognition, 22(2), 277-289. https://doi.org/10.1007/s10071-019-01244-7 (cited on page 12)

Ritchie, S. J., \& Bates, T. C. (2013). Enduring links from childhood mathematics and reading achievement to adult socioeconomic status. Psychological Science, 24(7), 1301-1308. https://doi.org/10.1177/0956797612466268 (cited on page 11)

Rivera, S. M., Reiss, A. L., Eckert, M. A., \& Menon, V. (2005). Developmental changes in mental arithmetic: Evidence for increased functional specialization in the left inferior parietal cortex. Cerebral Cortex, 15(11), 1779-1790. https://doi.org/10. 1093/cercor/bhi055 (cited on page 30)

Ross-Sheehy, S., Oakes, L. M., \& Luck, S. J. (2003). The development of visual shortterm memory capacity in infants. Child Development, 74(6), 1807-1822. https: //doi.org/10.1046/j.1467-8624.2003.00639.x (cited on page 15) 
Rubinsten, O., Dana, S., Lavro, D., \& Berger, A. (2013). Processing ordinality and quantity: Erp evidence of separate mechanisms. Brain and Cognition, 82(2), 201-212. https://doi.org/https://doi.org/10.1016/j.bandc.2013.04.008 (cited on page 31)

Rugani, R., Regolin, L., \& Vallortigara, G. (2008). Discrimination of small numerosities in young chicks. Journal of experimental psychology. Animal behavior processes, 34(3), 388-399. https://doi.org/10.1037/0097-7403.34.3.388 (cited on pages 24, 41)

Sarnecka, B. W., Goldman, M. C., \& Slusser, E. B. (2015, March 3). How counting leads to children's first representations of exact, large numbers. In R. Cohen Kadosh \& A. Dowker (Editors), The oxford handbook of numerical cognition (Pages 291-309).

Oxford University Press. https://doi.org/10.1093/oxfordhb/9780199642342.013. 011. (Cited on page 22)

Sarnecka, B. W., \& Lee, M. D. (2009). Levels of number knowledge during early childhood. Journal of Experimental Child Psychology, 103(3), 325-337. https://doi.org/10. 1016/j.jecp.2009.02.007 (cited on page 22)

Scholl, B. J. (2001). Objects and attention: The state of the art. Cognition, 80(1-2), 1-46. https://doi.org/10.1016/S0010-0277(00)00152-9 (cited on page 15)

Shaki, S., \& Fischer, M. H. (2014). Random walks on the mental number line. Experimental Brain Research, 232(1), 43-49. https://doi.org/10.1007/s00221-013-3718-7 (cited on page 41)

Shaki, S., Fischer, M. H., \& Petrusic, W. M. (2009). Reading habits for both words and numbers contribute to the snarc effect. Psychonomic Bulletin and Review, 16(2), 328-331. https://doi.org/10.3758/PBR.16.2.328 (cited on page 41)

Siegler, R. S. (2016). Magnitude knowledge: The common core of numerical development. Developmental Science, 19(3), 341-361. https://doi.org/10.1111/desc.12395 (cited on page 25)

Siegler, R. S., \& Booth, J. L. (2004). Development of numerical estimation in young children. Child Development, 75(2), 428-444. https://doi.org/10.1111/j.14678624.2004.00684.x (cited on page 23)

Siegler, R. S., \& Opfer, J. E. (2003). The development of numerical estimation: Evidence for multiple representations of numerical quantity. Psychological Science, 14(3), 237-243. https://doi.org/10.1111/1467-9280.02438 (cited on pages 22, 23)

Siegler, R. S., \& Ramani, G. B. (2009). Playing linear number board games-but not circular ones-improves low-income preschoolers' numerical understanding. Journal of 
Educational Psychology, 101(3), 545-560. https://doi.org/10.1037/a0014239 (cited on page 23)

Soylu, F., Lester Jr, F. K., Newman, S. D., Norton, A., \& Nurnberger-Haag, J. (2018). You can count on your fingers: The role of fingers in early mathematical development. Journal of Numerical Cognition, 4(1), 2363-8761. https://doi.org/10.5964/jnc. v4i1.85 (cited on pages 11,46 )

Soylu, F., Rivera, B., Anchan, M., \& Shannon, N. (2019). Erp differences in processing canonical and noncanonical finger-numeral configurations. Neuroscience Letters, 705, 74-79. https://doi.org/10.1016/j.neulet.2019.04.032 (cited on pages 44, 48-50)

Spelke, E. S. (2017). Core knowledge, language, and number. Language Learning and Development, 13(2), 147-170. https://doi.org/10.1080/15475441.2016.1263572 (cited on page 25)

Spelke, E. S., \& Kinzler, K. D. (2007). Core knowledge. Developmental Science, 10(1), 89-96. https://doi.org/10.1111/j.1467-7687.2007.00569.x (cited on page 15)

Stanley Jevons, W. (1871). The power of numerical discrimination. Nature, 3(67), 281-282. https://doi.org/10.1038/003281a0 (cited on pages 12, 13, 15, 18)

Sullivan, J., \& Barner, D. (2012). How are number words mapped to approximate magnitudes? Quarterly Journal of Experimental Psychology, 66(2), 389-402. https://doi.org/10.1080/17470218.2012.715655 (cited on page 25)

Sullivan, J., \& Barner, D. (2014). Inference and association in children's early numerical estimation. Child Development, 85(4), 1740-1755. https://doi.org/10.1111/cdev. 12211 (cited on page 29)

Temple, E., \& Posner, M. I. (1998). Brain mechanisms of quantity are similar in 5-year-old children and adults. Proceedings of the National Academy of Sciences of the United States of America, 95(13), 7836-41. https://doi.org/10.1073/pnas.95.13. 7836 (cited on pages 31, 32)

Trick, L. M., \& Pylyshyn, Z. W. (1994). Why are small and large numbers enumerated differently? a limited-capacity preattentive stage in vision. Psychological Review, 101(1), 80-102. https://doi.org/10.1037/0033-295X.101.1.80 (cited on page 15)

Tschentscher, N., Hauk, O., Fischer, M. H., \& Pulvermüller, F. (2012). You can count on the motor cortex: Finger counting habits modulate motor cortex activation evoked by numbers. NeuroImage, 59(4), 3139-3148. https://doi.org/10.1016/j. neuroimage.2011.11.037 (cited on page 43) 
van Oeffelen, M. P., \& Vos, P. G. (1982). A probabilistic model for the discrimination of visual number. Perception \& Psychophysics, 32(2), 163-170. https://doi.org/10. 3758/BF03204275 (cited on page 19)

Wiese, H. (2003). Numbers, language, and the human mind. Cambridge University Press. (Cited on page 42).

Wilson, M. (2002). Six views of embodied cognition. Psychonomic Bulletin and Review, 9(4), 625-636. https://doi.org/10.3758/BF03196322 (cited on page 41)

Wilson, M. L., Hauser, M. D., \& Wrangham, R. W. (2001). Does participation in intergroup conflict depend on numerical assessment, range location, or rank for wild chimpanzees? Animal Behaviour, 61(6), 1203-1216. https://doi.org/10.1006/anbe.2000.1706 (cited on page 14)

Wood, G., Willmes, K., Nuerk, H.-C., \& Fischer, M. H. (2008). On the cognitive link between space and number: A meta-analysis of the snarc effect. Psychology Science, 50(4), 489-525 (cited on page 41).

Wynn, K. (1990). Children's understanding of counting. Cognition, 36(2), 155-193. https: //doi.org/10.1016/0010-0277(90)90003-3 (cited on pages 21, 22, 25)

Wynn, K. (1992). Children's acquisition of the number words and the counting system. Cognitive Psychology, 24(2), 220-251. https://doi.org/10.1016/0010-0285(92) 90008-P (cited on pages 21, 22, 25)

Zhao, H., Chen, C., Zhang, H., Zhou, X., Mei, L., Chen, C., Chen, L., Cao, Z., \& Dong, Q. (2012). Is order the defining feature of magnitude representation? an erp study on learning numerical magnitude and spatial order of artificial symbols (E. Akyürek, Editor). PLoS ONE, 7(11). https://doi.org/10.1371/journal.pone.0049565 (cited on pages 33,34 ) 



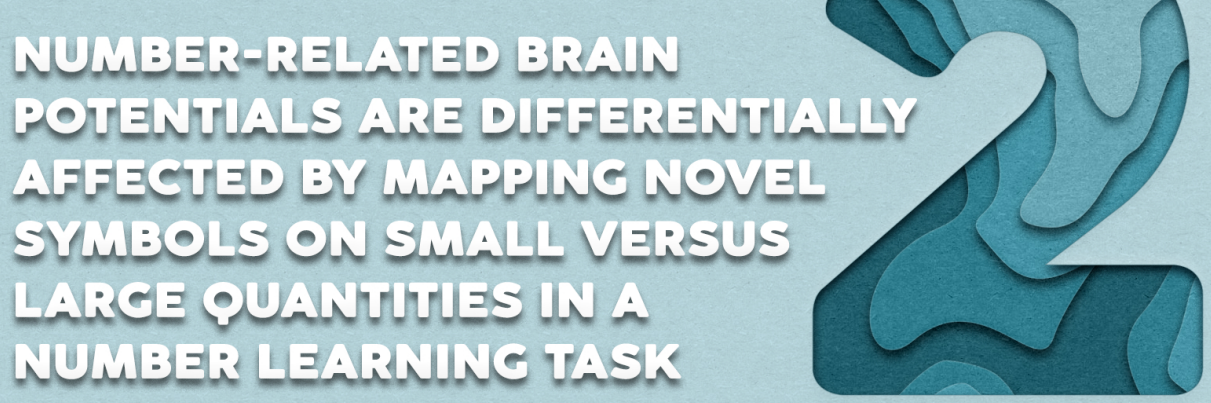

\section{1 Abstract}

The nature of the mapping process that imbues number symbols with their numerical meaning — known as the symbol-grounding process — remains poorly understood and the topic of much debate. The aim of the present study was to enhance insight into how the non-symbolic-symbolic number mapping process and its neurocognitive correlates might differ between small (1-4; subitizing range) and larger (6-9) numerical ranges. Hereto, twenty-two young adults performed a learning task in which novel symbols acquired numerical meaning by mapping them onto non-symbolic magnitudes presented as dot arrays (range 1-9). Learning-dependent changes in accuracy and reaction time provided evidence for successful novel symbol-quantity mapping in the subitizing (1-4) range only. Corroborating these behavioral results, the number processing related $\mathrm{P} 2 \mathrm{p}$ component was only modulated by the learning/mapping of symbols representing small numbers 1-4. The symbolic-N1 amplitude increased with learning independent of symbolic numerical range, but dependent on the set-size of the preceding dot array; it only occurred when mapping on 1-4 item dot arrays that allow for quick retrieval of a numeric value, on the basis of which, with learning, one could predict the upcoming symbol causing perceptual expectancy violation when observing a different symbol. These combined results suggest that exact nonsymbolic-symbolic mapping is only successful for small quantities 1-4 from which one can readily extract cardinality. Furthermore, we suggest that the P2p reflects the processing stage of first access to-, or retrieval of, numeric codes and might in future studies be used as a neural correlate of non-symbolic-symbolic mapping/symbol learning.

Corresponding reference: van den Berg, F. C. G., de Weerd, P., \& Jonkman, L. M. (2020). Number-related brain potentials are differentially affected by mapping novel symbols on small versus large quantities in a number learning task. Journal of cognitive neuroscience, 32(7), 1263-1275. 


\subsection{Introduction}

Several species have been shown to distinguish between small sets of objects (Agrillo et al., 2012; Gross et al., 2009; Xia et al., 2000), but only humans develop a symbolic number system in which each symbol (e.g. number word or Arabic digit) represents a specific quantity. Acquisition of the symbolic number system is a long process that involves intensive training; it takes children about two years (between 2 and 4 years of age) to acquire the symbolic representations for magnitudes 1-4 (also called the subitizing range; Wynn, 1990, 1992). The importance of developing accurate symbolic representations in early childhood is underlined by its positive correlation with arithmetic skills later in life (Duncan et al., 2007; Göbel et al., 2014; Holloway and Ansari, 2009; Siegler and Ramani, 2009). Yet, the mechanism underlying the mapping process that imbues number symbols with their numerical meaning — known as the symbol-grounding process — remains poorly understood and the topic of much debate (Carey, 2009; Dehaene, 2001; Le Corre and Carey, 2007; Leibovich and Ansari, 2016; Piazza, 2010; Reynvoet and Sasanguie, 2016; Siegler, 2016; Spelke, 2017; Sullivan and Barner, 2012). Here, we aimed to get more insight into the symbolic mapping process and its neurocognitive correlates by measuring adults' EEG while they performed a trial-and-error learning task in which they had to map meaningless novel symbols onto a specific magnitude (range 1-9) presented as dot arrays.

Number processing has predominantly been studied using number comparison paradigms. In such paradigms, participants decide which of two stimuli represents the largest quantity. The task can comprise symbolic and/or non-symbolic stimuli (e.g. arrays of dots), and can entail comparisons between multiple simultaneously presented stimuli or between a target and a fixed internal reference number (for example the number 5). A well-documented behavioral phenomenon in such tasks is the numerical distance effect (NDE; Moyer and Landauer, 1967), referring to performance (reaction time, accuracy) becoming better when the numerical distance between two to-be-compared numerical stimuli gets larger. Dehaene $(1992,2001 ; 1990)$ has argued that the NDE arises as a result of numbers being represented spatially along a mental number line, along which representational precision or 'tuning' decreases with increasing quantity. On such an ordered mental number line, two representations can interfere with each other if their tuning distributions overlap, resulting in the NDE. 
Based on this literature, two different systems have been proposed to underlie the processing of non-symbolic numerosities (numbers of objects). The first being an Object Tracking System (OTS) for exact representation of smaller numbers of objects (four or smaller; Feigenson et al., 2004). This OTS is a pre-attentive limited capacity system that is able to track $\approx 4$ items in parallel. The second is the Approximate Number System (ANS) for making approximate estimations of the magnitude of larger numbers of objects (larger than four) without relying on language or symbols (Feigenson et al., 2004)). A quantity of four or less items (OTS) can be estimated very fast and without error (Lipton and Spelke, 2004; Piazza et al., 2011; Revkin et al., 2008; Trick and Pylyshyn, 1994). By contrast, estimations of quantities above four are less accurate, although the precision and robustness of representations of larger numbers does increase with development (Halberda and Feigenson, 2008). In addition to these two systems processing the magnitude of object sets, literate adults possess a third system: the Symbolic Number System (SNS), in which each number symbol (e.g., the word "seven" or the digit "7") represents a specific quantity (e.g., a set of 7 objects). While the non-symbolic OTS and ANS have been observed in various non-human species (Agrillo et al., 2012; Xia et al., 2000) and very young infants (Feigenson et al., 2002; Xu and Spelke, 2000), only humans develop the SNS (see for reviews on symbolic and non-symbolic representations: Nieder and Dehaene, 2009; Piazza, 2010). As mentioned above, the process by which one acquires the SNS is still poorly understood, and different proposals on how number symbols (such as Arabic numerals) might gain their numerical meaning have been put forward. For many years the dominant view has been that number symbols acquire their numerical meaning by direct mapping onto already existing non-symbolic representations of numbers in the ANS (Dehaene, 2001; Piazza, 2010). However, based on more recent findings and reviews of the number processing literature, several authors concluded that direct (exact) non-symbolic (ANS) symbolic mapping is not likely to apply for the full range of discrete quantities (Leibovich and Ansari, 2016; Reynvoet and Sasanguie, 2016; Siegler, 2016), and might only occur for small numerosities 1-4 (Carey, 2009; Reynvoet and Sasanguie, 2016). Several recent developmental studies have indeed shown that 3-4 year-old children acquired non-symbolic (dot arrays) - symbolic (number word/digit) mappings more easily for small (1-3) than for larger (4-9) numerosities, suggesting that children can only make use of a non-symbolic system for symbol mapping in the case of small quantities for which one can easily derive the cardinality (Benoit et al., 2013; Hurst et al., 2016; Lira et al., 2017). Sullivan \& Barner (2014) concluded that only the first six number words were directly (associatively) mapped onto their corresponding quantities in 5-7 year-old children. It has accordingly 
been suggested that once numerical representations for the first four symbols have been acquired, the learning of symbolic representations for larger quantities would rely on generalizing successor and cardinality principles without the need for direct non-symbolic to symbolic mapping (Carey, 2009; Reynvoet and Sasanguie, 2016; Siegler, 2016).

The electrophysiological correlates of the learning process by which non-symbolic to symbolic mapping occurs for numbers 1-4 has been studied in non-human primates (Diester and Nieder, 2007, 2010; Nieder et al., 2006), but in humans equivalent nonsymbolic to symbolic mapping studies are lacking. There are, however, numerous studies that have investigated the electrophysiological correlates of processing of already learned non-symbolic and symbolic numbers in the adult brain. In such studies, two early EventRelated brain Potential (ERP) components gained most attention, and have been linked to different number processing stages. The first is the N1, which peaks at approximately 150 ms post-stimulus above occipital-temporal electrodes. The second is the $\mathrm{P} 2 \mathrm{p}$, which is a posterior positivity following the N1 which, in adults, peaks around 200-300 ms after stimulus onset and is measured at parietal-occipital-temporal electrodes (Dehaene, 1996; Hyde and Spelke, 2009; Libertus et al., 2007; Rubinsten et al., 2013; Temple and Posner, 1998). Most of these number processing ERP studies have made use of number comparison paradigms, taking the latency at which numerical ratio or distance effects first occur in the ERP as the time at which numerical magnitude representations are accessed. This approach however has yielded inconsistent conclusions about whether one already has access to numerical representations in the early processing window reflected by the $\mathrm{N} 1$, or whether such access is available only later in the $\mathrm{P} 2 \mathrm{p}$ processing window. Although distance or ratio effects have been reported on the N1 in non-symbolic number comparison tasks, these were confounded by changes in visual properties of non-symbolic number stimuli, such as the size and density of dot arrays that vary with increasing quantity (Hyde and Spelke, 2009; Libertus et al., 2007; Soltész and Szucs, 2014). This is in line with other studies showing that N1 topography can be modulated by stimulus format (e.g. number words versus Arabic digits; Dehaene, 1996; Park et al., 2014; Pinel et al., 2001; Turconi et al., 2004). 
Dehaene (1996) was the first to report a P2p amplitude decrease in adults in association with a decreased reaction time when increasing the numerical distance between an internal reference number (five) and a symbolic number stimulus (Arabic digit or number word representing numbers 1-9). This P2p numerical distance effect was lateralized to the right hemisphere and was not affected by stimulus format (digits or words). These ERP findings led Dehaene (1996) to identify the processing stage reflected by the P2p as the stage at which one has first access to semantic numerical (magnitude) representations. The decrease in P2p amplitude with increasing numerical distance between two to-bediscriminated numbers is thought to reflect the need for less effortful processing when there is less representational overlap. These number distance effects on the P2p were replicated in several other number comparison studies with children or adults (Libertus et al., 2007; Pinel et al., 2001; Temple and Posner, 1998). Modulation of P2p by the distance or ratio between number stimuli was also reported in a passive viewing paradigm that did not require active number comparisons (Hyde and Spelke, 2009). A study by Rubinsten, Dana, Lavro, \& Berger (2013) showed that even when the primary task of the participants was to decide if three simultaneously presented dot-arrays (range 1 to 20) were in the correct numerical order, the $\mathrm{P} 2 \mathrm{p}$ was modulated by the absolute quantity (ratio) differences between the stimuli, whereas earlier components such as the P1 were only sensitive to ordinality. These consistent findings of number distance or ratio effects on the P2p amplitude across modalities and in passive viewing tasks as well as in tasks requiring active quantity comparisons corroborate the findings of Dehaene (1996), and makes the $\mathrm{P} 2 \mathrm{p}$ the best candidate for the processing stage where first numerical access to magnitude codes occurs.

The above reviewed studies have however investigated the effects of number processing on $\mathrm{N} 1$ and $\mathrm{P} 2 \mathrm{p}$ components in adult participants who already have fully matured symbolic number representations that may no longer rely on non-symbolic magnitude representations (Leibovich and Ansari, 2016; Lyons et al., 2015). It is thus not known whether the $\mathrm{N} 1$ and $\mathrm{P} 2 \mathrm{p}$ components are (differentially) modulated by the process of non-symbolic - symbolic mapping, through which novel symbols acquire their numerical meaning. To the best of the authors' knowledge, only two prior studies measured the effects of artificial number symbol learning on behavior as well as ERPs (Merkley et al., 2016; Zhao et al., 2012). In both studies adults acquired the numerical meaning of novel symbols by either mapping them onto dot arrays representing magnitudes (in both studies above 10) or by using ordinal information. To measure the effects of training, participants performed a number comparison task on the new symbols 
at different phases during learning (session effects), while monitoring the emergence of distance (ratio) effects on accuracy, reaction time and ERPs. In both studies, P2p amplitudes to the novel symbols decreased as those symbols gained numerical meaning, regardless of whether symbols were learned on the basis of magnitude or ordinal information. Furthermore, both studies found significant numerical distance effects on accuracy, RT and P2p amplitude, the latter being larger for numerically close than far symbols. Merkley et al. (2016) also found numerical distance effects on the N1 (close > far), whereas the N1 was not studied in Zhao et al. (2012). Concluding, both Zhao et al. (2012) and Merkley et al. (2016) showed that newly learned symbols can elicit numerical distance effects on the $\mathrm{P} 2 \mathrm{p}$ in a number comparison task, suggesting their association with a spatial (non-symbolic) representation of quantity. However, these studies did not report on ERPs during the mapping/learning task itself and only investigated symbols mapped onto quantities of 10 and higher that are processed by the ANS and are not likely to allow for exact mapping. These studies do thus not answer the question of whether non-symbolic - symbolic mapping affects learning and N1 and P2p components differently for small (1-4) versus large (6-9) number ranges (i.e. in- or outside the subitizing range).

\subsubsection{Present Study}

The current study will investigate how behavioral performance, as well as N1 and P2p amplitude change over time as novel symbols become increasingly more strongly associated with already acquired numerical representations in a trial and error learning task. The range of quantities used contained the subitizing range (1-4) and a non-subitizing range (6-9), allowing us to assess potential differences in the dynamic mapping for quantities inside and outside the subitizing range. It is hypothesized that one-to-one non-symbolic-symbolic mapping will only occur on trials in which the dot array automatically activates a specific magnitude with which the new symbol can subsequently be successfully associated on same trials and dissociated on different trials. Because the fast presentation rate will prohibit counting, exact symbol mapping is expected to only occur for low numbers (14) processed by the OTS. Dot arrays 6-9 are expected to only activate an approximate sense of number (ANS), which is expected to hinder the acquisition of exact numerical meaning of subsequently shown symbols. Based on this, it is hypothesized that symbol learning will be least successful on trials where dot arrays and symbols both represent a number from the 6-9 numerical range for which only approximate learning can take place. Because prior research points to the $\mathrm{P} 2 \mathrm{p}$ as the most likely neural correlate of the 
processing stage at which retrieval of magnitude codes takes place, modulation of the P2p (and not N1) is expected to occur with the emergence of exact symbolic representations reflecting easier access/retrieval of magnitude codes. Thus, symbol learning effects are hypothesized to primarily occur on the P2p and to be strongest for symbols in the small 1-4 number range for which exact mapping is possible. Only a learning paradigm like that used in the present study can disentangle effects of visual stimulus complexity and number magnitude processing on the $\mathrm{N} 1$ and $\mathrm{P} 2 \mathrm{p}$, because only the latter changes with learning while low-level stimulus differences are kept the same across learning blocks (see for similar approach in the language domain: McCandliss et al., 1997).

\subsection{Method}

\subsubsection{Participants}

Participants were twenty-two University students ( $\mathrm{M}_{\mathrm{age}}=22.3, \mathrm{SD}=3.7,20$ females). All received a monetary compensation. One participant did not complete the training due to difficulties in tolerating the EEG cap, and was removed from all analyses. The study was approved by the local Ethical Committee of the Faculty of Psychology and Neuroscience (ERCPN) at Maastricht University and all participants signed an informed consent form.

\subsubsection{Procedure}

All participants visited the lab on two consecutive days during which they performed several blocks of the nonsymbolic - symbolic mapping/learning task (see Figure 2.1). On the first day, two blocks of the symbol learning task were performed (each consisting of 144 trials), and a third block of 288 trials was performed the second testing day (for an elaborate description of the learning task see paragraph 2.3.3). The participants also performed a number comparison task with the novel symbols after each of the three learning blocks. Since this task however only involved deciding whether a presented symbol was smaller or larger than five (fixed internal reference number), the data from this task did not contribute to the main research question focused on the dynamic effects of the non-symbolic - symbolic mapping/learning process itself on behavior and ERPs, and were thus not reported on here. Finally, at the end of the second testing day the participants performed a number line placement task in which they had to place randomly ordered cards of the novel symbols on a horizontal number line beginning with the smallest number on the left side. All solutions were photographed for later scoring. 


\subsubsection{Symbol Leaning/Mapping Task}

The design of our non-symbolic - symbolic learning/mapping task was similar to that used in non-human primates by Diester and Nieder (2007). Figure 2.1 shows the nine symbols (A) and an example trial of the learning task (B). In each trial, a dot array representing one of the nine quantities 1-9 was shown after a fixation square, followed by one of the symbols to be associated with these quantities. The quantity of five was excluded in all subsequent analyses, which is in line with previous research (Dehaene, 1996; Libertus et al., 2007; Temple and Posner, 1998). To control for non-numerical factors associated with increasing non-symbolic quantity (such as dot size or surface area), dot diameter was fixed in half of the trials while total surface area occupied by the dots was fixed in the other half (by adapting dot size) according to the procedure suggested by Dehaene, Izard, and Piazza (2005). Furthermore, dot-array stimuli were randomized on surface area and dot size with a total of 32 possible configurations (16 for fixed size and surface). The here used nine novel symbols were selected from a larger set of 70 symbols (that were created in Powerpoint), on the basis of their discriminability (minimal overlapping features) and similar spatial occupation (so that they could be centered). Potential (unwanted) differences in discriminability between the nine chosen symbols were piloted by administering a 1-back task including the nine symbols to five participants (who did not participate in the later experiment). N-Back task results did not show any consistent differences in discrimination speed or accuracy between the nine symbols. In the learning task the symbols were presented in bold, italic, or a narrow typeface to reduce the possible use of alternative strategies. All stimuli were presented in black on a grey background using Presentation ${ }^{\circledR}$ (Version 18.0, Neurobehavioral Systems, Inc., Berkley, CA). Stimuli were presented centrally at a viewing distance of $57 \mathrm{~cm}$ and subtended about $5^{\circ}$ on the retina.

Participants had to indicate whether the magnitudes presented by the dot patterns and novel symbols matched/were the same (left button) or did not match/differed (right button). A block of 144 trials contained all possible pairings of dot arrays and symbols representing numbers in the 1-9 number range, with 72 trials in which paired dots and symbols represented a different quantity (each non-matching dot-symbol combination being presented only once) and 72 trials in which the dot array and the symbol represented the same quantity (same trials were repeated 8 times for each of the nine numbers within a 144 trial block). Trial presentation was fully randomized within 144-trial task blocks. Feedback was given after each correct (green square) and incorrect (red square) response 
(see Figure 2.1). Because behavioral learning effects were strongest between the first and the last learning block only these two learning blocks were included in the analyses. Since the number of trials in the last block of the learning/mapping task was twice as large as that in the first block, we repeated all below reported analyses with inclusion of only the first 144 trials of the last block (equalizing trial numbers). Since this did not change our results, we decided to keep the 288 trials in the last learning block to reduce signal/noise ratios and improve model fits. In the ERP analyses only different trials (irrespective of response accuracy) were included to adequately capture ERP and response time variations due to improvements in dot-symbol mappings across learning sessions, as was the purpose of the study. This also equalized the number of trials and signal/noise ratios between learning task blocks (because this is a learning task with gradually increasing accuracy, including only correctly responded trials would have yielded large differences in trials for ERP averaging in the first versus the last learning block).

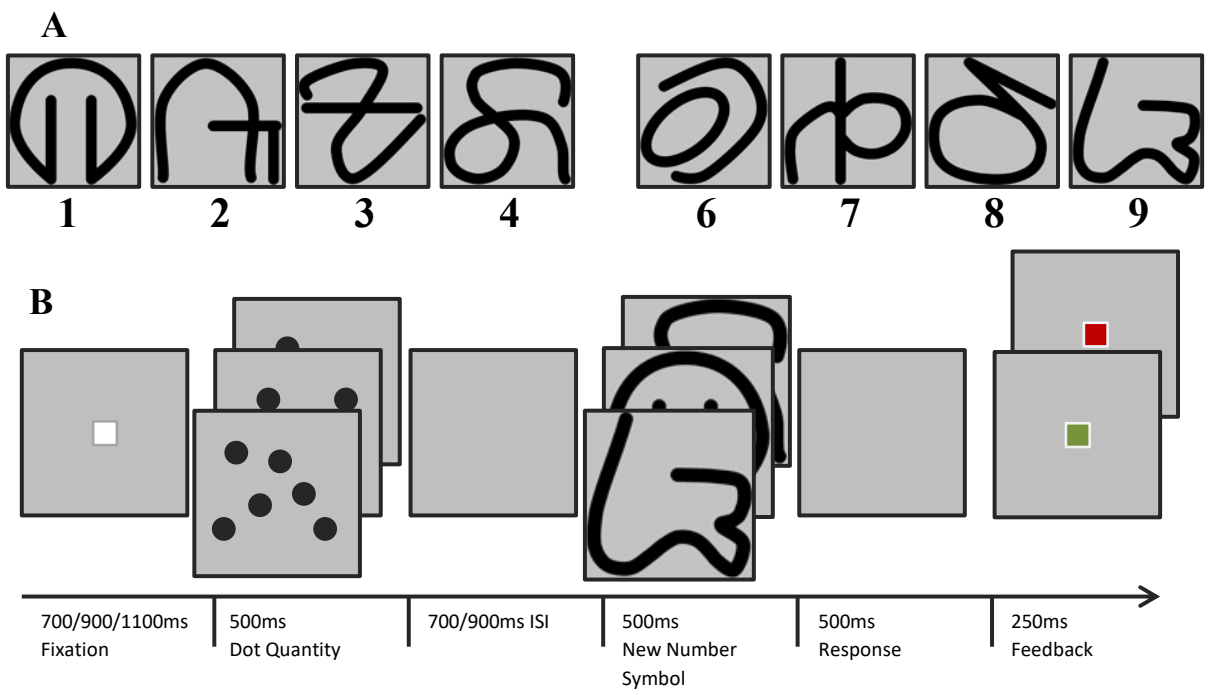

Figure 2.1: (A) The novel symbols representing cardinalities 1-9. (B) Example of a learning task trial: Participants were presented with a dot pattern between 1 and 9 followed by one of the novel number symbols, each symbol having a fixed association with a specific cardinality (see A). Participants had to indicate by button press if the presented symbol represented the same cardinality as the dot pattern (same trials) or a different cardinality (different trials) after which they received feedback (correct/wrong response). 


\subsubsection{EEG/ERP acquisition and analyses}

EEG data was recorded using a 64-channel electrode cap $(\mathrm{Ag} / \mathrm{AgCl})$ and using the BrainAmp amplifier system (Brain products $\mathrm{GmbH}$, Munich, Germany) and Brain Vision software. The data were filtered online at $0.01-225 \mathrm{~Hz}$ and continuously sampled at a rate of $500 \mathrm{~Hz}$. The left mastoid (A1) served as online reference (A2 measured as active electrode) and POz served as ground. Horizontal and vertical EOG was measured by electrodes placed on respectively the outer canthus of each eye and above/below the left orbit. All electrode impedances were kept below 5-10 kOhm.

EEG data was processed using EEGlab/Matlab 2014a. The data was resampled offline to $250 \mathrm{~Hz}$ and re-referenced to the average signal. A band-pass filter of $0.1 \mathrm{~Hz}$ to $70 \mathrm{~Hz}$ was applied to the data before execution of Independent Component Analysis (ICA) for removal of horizontal eye movements and blinks. The ocular artifact-free data was filtered using a $30 \mathrm{~Hz}$ Low-pass filter, after which the data was epoched (based on the dot-quantity and symbol-quantity event-codes) into $500 \mathrm{~ms}$ pre-stimulus and $1000 \mathrm{~ms}$ post-stimulus windows. Baseline correction was performed using the $500 \mathrm{~ms}$ pre-stimulus interval. After this, remaining EEG artifacts were removed by applying an automatic artifact detection procedure rejecting trials with activity exceeding a $\pm 75 \mu \mathrm{V}$ threshold.

Visual inspection of the Topography Maps and ERPs identified a bilateral cluster of Parietal- Occipital electrodes of interest (see Figure 2.3). These included the left P3, P5, $\mathrm{P} 7, \mathrm{PO} 3$, \& $\mathrm{PO} 7$ electrodes and the right P4, P6, P8, PO4, \& PO8 electrode sites. Based on the number-processing ERP literature (see introduction) and inspection of the grand average ERP signals of different conditions and sessions, two analysis windows were identified: 130-185ms (N1), and 210-250ms (P2p; see Figure 2.3). Mean amplitude scores within these windows were entered in the statistical analyses.

\subsubsection{Statistical Analysis}

To investigate whether performance and ERPs were differently affected by non-symbolic symbolic mapping of small numbers (1-4) versus larger numbers 6-9 (see introduction), all 'different' trials (where dot array and symbol represented a different quantity) were split up in 2x2 trial categories based on Dot quantity (Small, 1-4 or Large, 6-9) and Symbol quantity (Small, 1-4 or Large, 6-9). Behavioral dependent measures were mean accuracy and mean reaction time (in the RT analysis trials with RT $<150$ or $>1000$ ms 
were excluded). Neural dependent variables were N1 and P2p amplitude. For dependent measures reaction time, N1, and P2p Amplitude, we constructed Linear Mixed Models. Accuracy data was analyzed using a Generalized Estimation Equation (GEE) model due to severe violations of normality. All analyses included within-subject factors Hemisphere (2 levels: Left, Right), Session (2 levels: Session 1 and Session 3), Dot-Quantity (2 levels: Small1-4 and Large6-9), and Symbol-Quantity (2 levels: Small1-4 and Large6-9). To be able to check whether the participants acquired more accurate non-symbolic - symbolic mappings of small symbols 1-4 (subitizing range) than larger symbols (6-9) an extra 2 x 2 analysis with factors Session $x$ Symbol-Quantity was conducted for accuracy and RT on same trials where dot arrays and symbols represented the same magnitude.

The N1 model was constructed using within-subject factors Hemisphere, Session, DotQuantity, and Symbol-Quantity. Various models were tested, including random Intercept and random slopes for Session, Dot, and Symbol factors. None of these models had a better fit than the fixed effects model. Non-significant higher order interactions were removed in a step-wise manner starting with the four-way interaction Session*Hemisphere*DotQuantity*Symbol-Quantity $(p=.281)$, followed by the three-way interaction between Hemisphere*Dot-Quantity*Symbol-Quantity $(p=.969)$ and Session*Hemisphere*DotQuantity $(p=.359)$. The Session*Dot-Quantity*Symbol-Quantity interaction was kept based on theoretical expectations and all two-way interactions also remained in the model. The final model used for the N1 analyses (reported below) was a fixed effects model using an Unstructured Covariance Matrix for repeated measures. Eight observations (single data-points within participants) were removed as outliers based on their standardized residuals. The resulting model showed a significantly better fit.

The P2p model was constructed using the same within-subject factors and method as the N1 analysis. Non-significant higher order interactions were removed in a step-wise manner starting with the four-way interaction Session*Hemisphere*Dot-Quantity*SymbolQuantity $(p=.304)$, followed by the three-way interaction between Hemisphere*DotQuantity*Symbol-Quantity ( $p=.853)$, Session*Hemisphere*Symbol-Quantity $(p=.311)$, and Session*Hemisphere*Dot-Quantity $(p=.493)$. The Session*Dot-Quantity*SymbolQuantity interaction was kept based on theoretical expectations and all two-way interactions also remained in the model. The final model used for the P2p analyses (reported below) was a fixed effects model using an Unstructured Covariance Matrix for repeated measures. A single observation was removed as an outlier without significantly altering the fit of the model. 


\subsection{Results}

\subsubsection{Novel Symbol Learning Task}

Table 1 depicts all mean Accuracy, Reaction Time (RT), and N1, P2p amplitude data for the different Dot-Symbol combinations in the two learning task blocks (hereafter called sessions) on different response trials in the symbol learning task. The table also shows mean accuracy and RT data on same response trials. Learning curves for accuracy and reaction time data in the different learning task blocks across low and high number ranges are depicted in Figure 2.2 Average ERPs and topo-plots are shown in Figure 2.3.

Table 1: Means and Standard Deviations (between brackets) for behavioral and ERP data in the four different Dot-Symbol conditions on different response trials (rows with Dots 1-4 and Dots 6-9) and behavioral data on same response trials in learning sessions 1 and 3.

\begin{tabular}{lllrr}
\hline & & & Symbols 1-4 & \multicolumn{1}{c}{ Symbols 6-9 } \\
\hline Accuracy & Session 1 & Dots 1-4 & $54.96 \%(17.98 \%)$ & $59.13 \%(15.57 \%)$ \\
& & Dots 6-9 & $58.99 \%(16.45 \%)$ & $55.06 \%(14.82 \%)$ \\
& & Same Trials & $63.58 \%(18.37 \%)$ & $38.43 \%(14.35 \%)$ \\
& Session 3 & Dots 1-4 & $82.97 \%(11.89 \%)$ & $93.58 \%(10.34 \%)$ \\
& & Dots 6-9 & $83.14 \%(14.25 \%)$ & $65.99 \%(14.83 \%)$ \\
& & Same Trials & $89.49 \%(13.45 \%)$ & $61.77 \%(20.33 \%)$ \\
\hline Reaction Times & Session 1 & Dots 1-4 & $683.19(100.31)$ & $698.26(103.27)$ \\
& & Dots 6-9 & $706.51(108.73)$ & $729.50(91.20)$ \\
& & Same Trials & $666.82(115.98)$ & $706.18(108.33)$ \\
& & Dession 3 & $618.53(77.62)$ & $590.99(87.47)$ \\
& & Dots 6-9 & $635.13(92.58)$ & $627.55(78.88)$ \\
& & Same Trials & $553.31(73.59)$ & $630.34(90.83)$ \\
\hline N1 (130-185 ms) & Session 1 & Dots 1-4 & $-4.33(3.44)$ & $-2.90(2.77)$ \\
& & Dots 6-9 & $-3.66(2.03)$ & $-2.52(2.62)$ \\
& Session 3 & Dots 1-4 & $-5.42(2.82)$ & $-4.09(2.28)$ \\
& & Dots 6-9 & $-3.31(2.82)$ & $-2.54(2.88)$ \\
\hline P2p (210-250 ms) & Session 1 & Dots 1-4 & $2.75(3.16)$ & $3.37(2.88)$ \\
& & Dots 6-9 & $3.67(2.26)$ & $3.41(2.25)$ \\
& Session 3 & Dots 1-4 & $1.87(2.52)$ & $2.73(2.20)$ \\
& & Dots 6-9 & $2.66(2.93)$ & $3.70(2.67)$ \\
\hline P2p (210-250 ms) & Session 1 & Dots 1-4 & $3.45(2.72)$ & $3.36(2.68)$ \\
& & Dots 6-9 & $3.93(2.22)$ & $3.13(2.44)$ \\
& Session 3 & Dots 1-4 & $3.11(2.37)$ & $3.41(2.55)$ \\
& & Dots 6-9 & $2.72(2.72)$ & $3.28(2.84)$ \\
\hline
\end{tabular}

Values shown are estimated marginal means. a: P2p analysis results with $\mathrm{N} 1$ amplitude entered as covariate (estimated values for $\mathrm{N} 1=-3.5066 \mu \mathrm{V}$ ) 


\section{Behavioral Data}

\section{Accuracy and Reaction Time (RT) effects on same response trials.}

Accuracy. The Accuracy analysis yielded a significant main effect of Session $\left(\chi_{1}^{2}=69.74\right.$, $p<.001$ ), showing an overall improvement in accuracy of $24.63 \%$ from session 1 to 3 , regardless of whether symbols represented a number within the Small1-4 or Large6-9 number range ( $\mathrm{M}_{\text {diff-session }}$ Small was $25.92 \%$ and $\mathrm{M}_{\text {diff-session }}$ Large was $23.34 \%$ ). A main Symbol-Quantity effect $\left(\chi_{1}^{2}=94.34, p<.001\right)$ showed that accuracy was $26.43 \%$ higher for Symbols S-4 $_{1}$ than for Symbols $6-9$ (Session 1: 25.14\%; Session 3: 27.72\%), indicating that Symbols $_{1-4}$ already acquired numerical meaning during the first 144 trials in session 1 (see Figure 2.2). There was no significant Session*Symbol-Quantity interaction $\left(\chi_{1}^{2}=\right.$ $0.17, p=.680)$.

Reaction time. Significant main effects of Session $(F[1,22.12]=21.52, p<.001)$ and Symbol-Quantity $(F[1,22.22]=60.32, p=.001)$ showed that responses were significantly faster in session $3\left(t[22.12]_{\text {Session1-Session } 3}=4.64, p<.001\right)$ and for Small 1-4 $_{\text {Symbols }}$ $\left(t[22.22]_{\text {Small-Large }}=-7.77, p=.001\right)$. The Session*Symbol-Quantity interaction was marginally significant $(F[1,20.91]=3.22, p=.087)$.

\section{Accuracy effects on different response trials.}

This analysis yielded significant main effects of Session $\left(\chi_{1}^{2}=104.56, p<.001\right)$ and DotQuantity $\left(\chi_{1}^{2}=23.63, p<.001\right)$, with an accuracy increase from session 1 to 3 and higher accuracy in trials where symbols were preceded by Small $_{1-4}$ compared to Large 6-9 $_{9}$ dot arrays. These main effects were qualified by two-way interactions of Session*Dot-Quantity $\left(\chi_{1}^{2}=15.16, p<.001\right)$ and Dot-Quantity*Symbol-Quantity $\left(\chi_{1}^{2}=23.55, p<.001\right)$, and a three-way Session*Dot-Quantity*Symbol-Quantity interaction $\left(\chi_{1}^{2}=15.15, p<.001\right)$. 

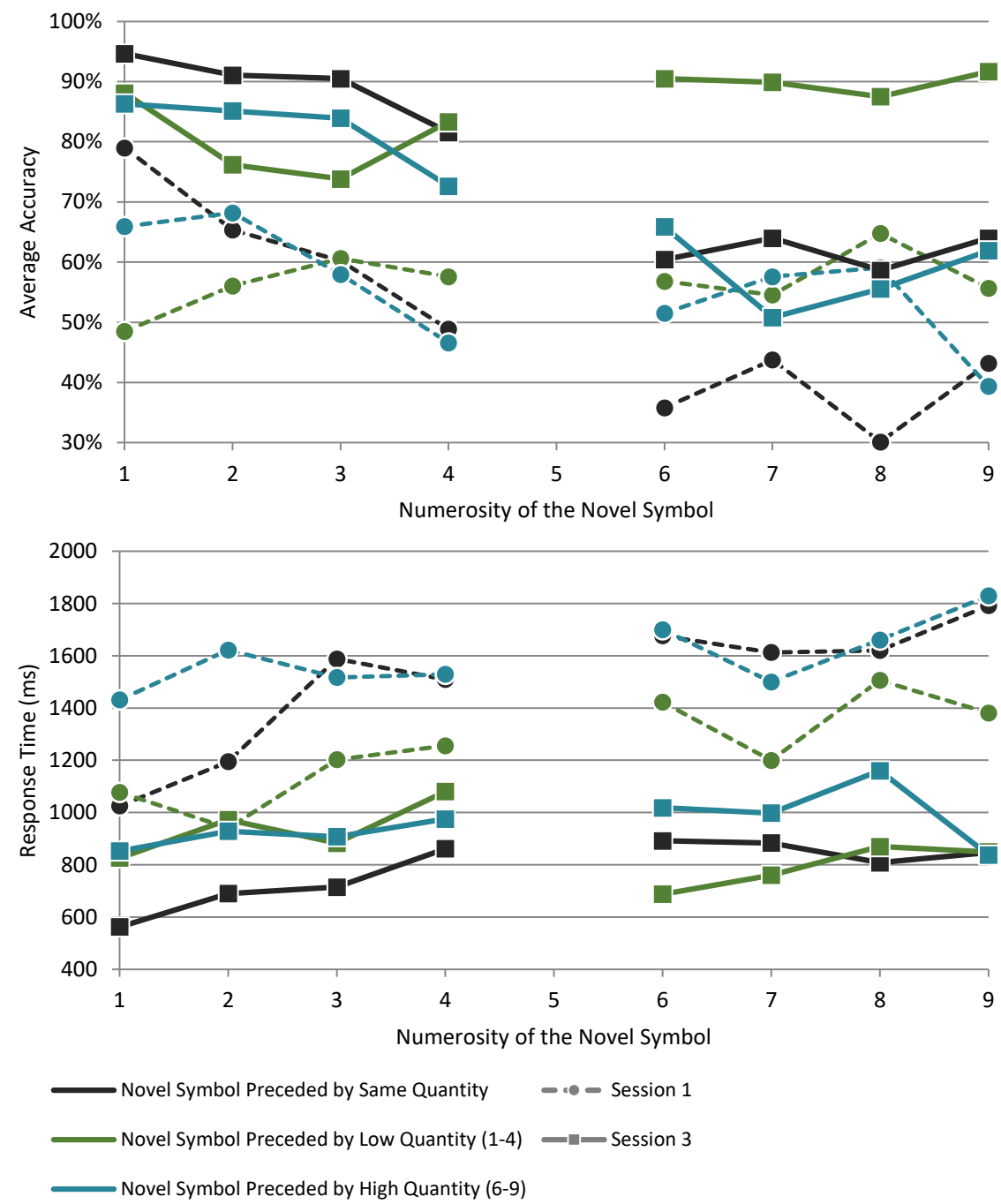

Figure 2.2: Behavioral Data from the Learning Task (LT) showing accuracy and reaction time to the novel symbols in the first (Session 1: dotted lines) and the last (Session 3, solid lines) learning task block in small (1-4) and large (6-9) number ranges. Symbols preceded by a dot quantity equal to the symbol (Same trials) are shown in black. Symbol processing on different trials, in which symbols were preceded by dot-arrays that did not match in quantity, were split up in: 1) novel symbols preceded by a dot array from the small (1-4) number range (green lines) or 2) novel symbols preceded by a dot array from the large (6-9) number range (blue lines). For novel symbols 1-4, same trials (shown in black) show stronger training gain in accuracy and speed of responding than Different trials (shown in green/blue). For symbols 6-9, this effect is reversed with higher training gain in accuracy and response time on different than same trials, but only when symbols 6-9 had to be mapped onto a preceding dot pattern depicting a numerosity in the low 1-4 number range. Both effects match with exact quantity - symbol mapping for only the first four symbols. 
Further testing of the three-way Session*Dot-Quantity*Symbol-Quantity interaction (Sidak corrected comparisons) showed significant Session effects (accuracy increases with learning) for all Symbol and Dot levels (all p-values $\leq .001$ ). A subsequent analysis was executed on the difference scores (Session 3 - Session 1), showing a significant DotQuantity*Symbol-Quantity interaction $\left(\chi_{1}^{2}=8.61, p=.003\right)$. Further (Sidak corrected) comparisons revealed this to be due to a smaller learning effect in trials in which both dot arrays and symbols represented a number in the high 6-9 number range than in any of the other Dot-Symbol trial combinations (Dots6-9-Symbols $6-9$ vs Dots6-9-Symbols $1-4$ : $t(1)$ $=2.86, p=.025$; Dots6-9-Symbols $6-9$ vs Dots ${ }_{1-4}$-Symbols $_{6-9}: t(1)=5.05, p<.001$; and Dots $_{6-9}-$ Symbols $_{6-9}$ vs Dots $_{1-4}-$ Symbols $\left._{1-4}: t(1)=2.60, p=.054\right)$.

\section{Reaction Time (RT) effects on different response trials.}

Main effects of Session $(F[1,17.60]=32.46, p<.001)$ and Dot-Quantity $(F[1,15.64]=$ 15.20, $p=.001)$ were found; RT was significantly faster in session 3 than 1 $\left(t[17.60]_{\text {Session } 1-S e s s i o n} 3=-5.70, p<.001\right)$ and in trials in which symbols were preceded by Small $_{1-4}$ compared to Large $6-9$ Dot arrays $\left(t[15.64]_{\text {Small-Large }}=-3.90, p=.001\right)$.

A two-way Session*Symbol-Quantity $(F[1,19.34]=8.80, p=.008)$ interaction was further explored by performing pairwise comparisons (Sidak Corrected). Testing of the Symbol-Quantity effect per level of Session showed no significant differences in RT between Small $_{1-4}$ and Large $6-9$ Symbols in Session $1\left(t[19.63]_{\text {Small-Large }}=-1.64, p=\right.$ .118), whereas RT was significantly slower for Small S $_{-4}$ than Large $6-9$ Symbols in Session $3\left(t[20]_{\text {Small-Large }}=2.59, p=.018\right)$. Session effects were significant for both Small $_{1-4}$

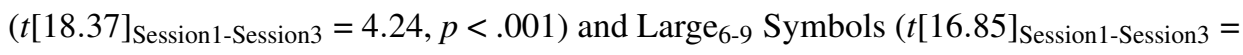
$6.28, p<.001)$, with the largest difference for the latter.

\section{Event-Related Potentials (ERPs)}

N1 amplitude (130-185 ms). The N1 analysis yielded significant main effects of DotQuantity $(F[1,19.98]=29.23, p<.001)$ and Symbol-Quantity $(F[1,15.07]=17.41, p=$ $.001)$; the $\mathrm{N} 1$ amplitude in response to symbols was larger when they were preceded by a small (1-4 item) dot array than when preceded by a large (6-9 item) dot array. Furthermore, small Symbols $_{1-4}$ elicited higher N1 amplitude than large Symbols6-9. These main effects were qualified by a two-way interaction of Session*Dot-Quantity $(F[1,18.42]=13.74, p=$ $.002)$ and a three-way interaction of Session*Hemisphere*Symbol-Quantity $(F[1,20.30]=$ $7.44, p=.013)$. 

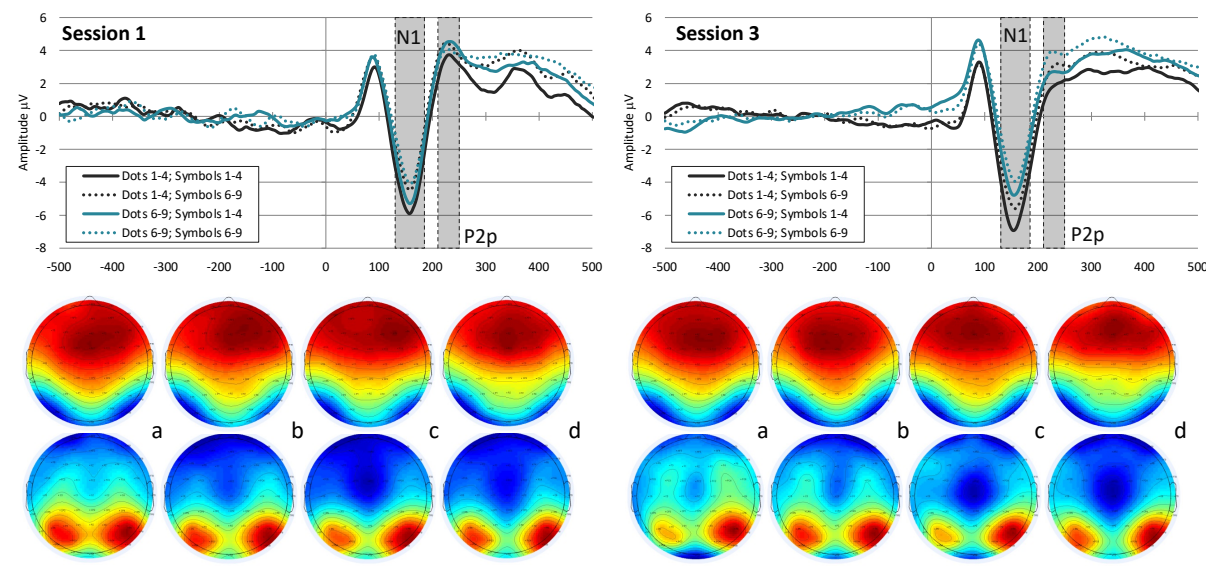

Figure 2.3: Grand Average ERP's (averaged over left and right parietal-occipital hemisphere clusters) and Topography plots (Top: N1; Bottom: P2p) in the four Dot-Symbol categories and the first session (left) and the last session (session 3; right) of the symbol learning task. a) Small-Dots ${ }_{1-4} /$ Small-Symbol $_{1-4}$, b) SmallDots $_{1-4} /$ Large-Symbol $_{6-9}$, c) Large-Dots $6-9 /$ Small-Symbol $_{1-4}$, d) Large-Dots $6-9$ /Small-Symbol ${ }_{1-4}$.

The two-way Session*Dot-Quantity interaction showed a significant Session effect (larger N1 amplitude in Session 3 than Session 1) for trials where Symbols were preceded by small dot arrays (1-4 items) $\left(t[19.87]_{\text {Session1-Session3 }}=2.52, p=.020\right)$, but not for trials on which symbols were preceded by large (6-9 item) dot arrays $\left(t[19.10]_{\text {Session1-Session3 }}=\right.$ $0.54, p=.599 ;$ see Table 1 and Figure 2.3). Follow-up tests of the three-way Session*Hemisphere*Symbol-Quantity interaction revealed no significant Session*Symbol-Quantity interactions in the left nor right hemisphere (left: $F[1,20]=$ $0.05, p=.942$; right: $F[1,17.12]=1.11, p=.306)$.

There were no other significant main effects (Session: $F[1,19.81]=1.32, p=.265$, and Hemisphere: $F[1,17.53]=0.49, p=.493$ ) nor interaction effects (Session*Hemisphere: $F[1,17.87]=0.18, p=.681$, Session*Symbol-Quantity: $F[1,19.55]=0.68, p=.419$, Hemisphere*Dot-Quantity: $F[1,17.94]=0.43, p=.518$, Hemisphere*Symbol-Quantity: $F[1,20.33]=0.19, p=.667$, Dot-Quantity*Symbol-Quantity: $F[1,20.02]=0.89, p=.356$, and Session*Dot-Quantity*Symbol-Quantity: $F[1,20.19]=0.56, p=.463)$. 
P2p amplitude (210-250 $\mathbf{~ m s ) . ~ T h e ~} \mathrm{P} 2 \mathrm{p}$ analysis yielded significant main effects for Hemisphere $(F[1,19.89]=9.58, p=.006)$, Dot-Quantity $(F[1,19.95]=10.06, p=.005)$ and Symbol-Quantity $(F[1,18.37]=11.94, p=.003)$; the $\mathrm{P} 2 \mathrm{p}$ amplitude in response to the symbols was larger in the right than the left hemisphere, was highest when symbols were preceded by large (vs small) dot arrays and was higher to large (6-9) than small (1-4) Symbols. These main effects were qualified by a significant two-way interaction between Session*Symbol-Quantity $(F[1,19.45]=12.82, p=.002)$, further testing of which showed a significant Session effect (smaller P2p in Session 3 than Session 1) for small Symbols $1-4$ $\left(t[19.90]_{\text {Session1-Session3 }}=2.23, p=.037\right)$, but not for large Symbols $6-9$ $\left(t[20]_{\text {Session1-Session3 }}\right.$ $=-0.47, p=.642$; see Table 1 and Figure 2.3). Marginally significant $(p<.1)$ effects were found for the Hemisphere*Symbol interaction $(F[1,18.61]=3.52, p=.076)$ and the three-way Session*Dot-Quantity*Symbol-Quantity interaction $(F[1,18.64]=3.08, p=$ .096). No other effects were significant.

To verify that the above P2p effects were not driven by effects on the preceding $\mathrm{N} 1$, a second $\mathrm{P} 2 \mathrm{p}$ model was run including the $\mathrm{N} 1$ amplitude as a covariate (keeping it constant at the overall N1 mean of $-3.51 \mu \mathrm{V}$ ), and showed that the N1 Amplitude covariate was significant $(F[1,83.24]=833.78, p<.001)$. After inclusion of the $\mathrm{N} 1$, all effects involving the Dot-Quantity factor were no longer significant (all p-values > .11), and were thus driven by earlier N1 effects. All effects involving Hemisphere and SymbolQuantity remained significant and/or only increased in strength after co-varying for N1 amplitude (Hemisphere: $F[1,18.32]=29.95, p<.001$; Hemisphere*Symbol-Quantity: $F[1,20.61]=6.56, p=.018$; and Session*Symbol-Quantity: $F[1,19.30]=21.20, p<$ $.001)$. No other effects were significant. Further testing of the Hemisphere*SymbolQuantity interaction showed significantly higher P2p amplitude at the right than the left hemisphere in response to both small Symbols ${ }_{1-4}\left(t[18.40]_{\text {Left-Right }}=-6.99, p<.001\right)$ and large Symbols6-9 $\left(t[18.64]_{\text {Left-Right }}=-3.93, p=.001\right)$, but this effect was slightly more pronounced for small Symbols $1-4$. Further testing of the two-way Session*SymbolQuantity interaction showed a significant Session effect (smaller P2p in Session 3 than Session 1) for small Symbols ${ }_{1-4}\left(t[20.16]_{\text {Session1-Session3 }}=2.70, p=.014\right)$, but not for large Symbols $_{6-9}\left(t[19.68]_{\text {Session1-Session3 }}=-0.32, p=.749\right)$. 


\subsubsection{Number Line Placement (ordering) Task with the Novel Symbols}

An exact position score was computed for all participants by assigning one point for each correctly placed symbol on the number line. A Wilcoxon Signed Ranks Test, comparing Low (1-4) and High (6-9) number ranges, showed significant differences in the exact position score $(\mathrm{Z}=-2.51, p=.012, \mathrm{r}[21]=-.55)$, with mean position scores being $3.57(S D=1.12 ; \operatorname{Mdn}=4 ; \operatorname{Min}=0 ; \operatorname{Max}=4)$ for the 1-4 number range and $2.76(S D=$ 1.30; $\operatorname{Mdn}=2 ; \operatorname{Min}=0 ; \operatorname{Max}=4$ ) for the 6-9 number range. These data confirm lower acquisition of exact numerical meaning for higher symbols as was also shown by the behavioral data in the learning task. Participants were also questioned about a) specific associations with symbols, b) whether some symbols were easier to learn than others, and c) whether specific strategies were used. There we no obvious patterns in symbol associations (question 1), while 8 participants (34.78\%) in response to questions 2 and 3 indicated in some form that the smaller range (1-4) was easier to learn. This was based on responses such as "trial and error and then I tried to remember the first 4 and the rest by chance" and "I tried to remember the symbols belonging to digits 1-4, since those I could see quickly enough for an exact digit". So, mostly, participants were not aware that in their learning low-range numbers had a special status.

\subsection{Discussion}

The aim of the present study was to get more insight into how the non-symbolic-symbolic mapping process and its neurocognitive correlates might differ between low (1-4) and high (6-9) numerical ranges. Previous studies suggest that non-symbolic-symbolic mapping will be most successful between small $(<4)$ arrays of objects and small digits because one can in this case make use of non-symbolic systems (OTS) for representing individuals of small arrays, allowing for exact, one-to-one, mapping. Without counting, such quick one-to-one mapping is suggested to be impossible for arrays of objects above the subitizing range for which enumeration is approximate, based on estimation (Benoit et al., 2004; Huang et al., 2010; Le Corre and Carey, 2007; Le Corre et al., 2006). To investigate this, adults' EEG was measured during performance of a trial-and-error symbol learning task in which they had to acquire the numerical meaning of novel symbols representing a small (1-4) versus a larger (6-9) number by mapping them onto their non-symbolic counterparts (presented as arrays of dots). 
The behavioral (accuracy and reaction time) data indeed provided evidence for easier mapping/better learning of symbols representing magnitudes 1-4 in the subitizing range. A three-way interaction effect showed that on trials where dot array and subsequent symbol represented a different magnitude (and required a 'different' response), accuracy increased across learning sessions for all four small/large dot-symbol combinations, but this accuracy increase was significantly lowest (only from 55\% in session 1 to $66 \%$ in session 3 ) when both dots and symbols represented a numerical value in the large (6-9) number range. In the other three categories accuracy increased much more strongly from 55-59\% in session 1 to $82-93 \%$ in session 3 . This can be explained by the fact that numerosities for dot-arrays in the low range 1-4 can be readily extracted by subitizing, leading to fast retrieval of an exact numeric representation/code from memory on which novel symbols (1-4) can be easily and exactly mapped in a one-to-one fashion. Support for successful exact mapping of only novel symbols 1-4 was further confirmed by analyses of behavioral data on trials where dots and symbol represented the same magnitude (same trials) and also by data from the number line placement task, showing almost perfect placement accuracy for symbols representing numerosities 1-4, whereas this was much lower for symbols 6-9. An accurate "same" judgment in the learning task requires knowing the exact numerosity belonging to the novel symbol (one-to-one mapping), which was hypothesized to be only possible for dot arrays 1-4 for which a numerical code could be readily extracted. Indeed, symbols 1-4 showed a significantly larger increase in mean accuracy across learning sessions (from 64\% in the first session to $89 \%$ in the last session) than symbols 6-9, the latter showing a mean accuracy increase from 38 to only $62 \%$. The development of exact numeric representations for symbols 1-4 in turn made it easier to decide that small-dot arrays (1-4) and large symbols (6-9) represent a different numerosity, explaining the large accuracy increase from session 1 to 3 in this trial category.

The time to decide whether dots and novel symbols represented a different numerosity decreased across learning sessions for all symbols, but a significant two-way session $\mathrm{x}$ small/large symbol interaction effect showed no significant difference in response times between small/large symbols in session 1, whereas in session 3 response times were significantly slower (about $40 \mathrm{~ms}$ ) for symbols in the small (1-4) than large (6-9) number range, independent of whether they were preceded by small or large dot arrays. One likely explanation for this difference is that with the increase in accurate mapping of the symbols representing numerosities $1-4$, it will take more time to retrieve their unique numerical codes from memory in order to decide whether the symbol and dot array represent a different (or the same) numerosity as was required in the present learning task. In the case 
a symbol cannot be mapped onto one specific numerosity, as in the case of a numerosity outside the subitizing range for which no exact numerical code can be extracted without counting (for which the presentation time was too short in the current task), one has to guess/approximate its numerical value which is a faster process than retrieving an exact numerical code from memory.

The current learning task paradigm also allowed us to investigate whether two early ERP components (N1 and P2p), that in earlier studies have been linked to the processing stage at which one has first numerical access to analog numerical information (Dehaene, 1996; Hyde and Spelke, 2009; Libertus et al., 2007; Rubinsten et al., 2013; Temple and Posner, 1998), were differentially modulated by number symbol learning in our nonsymbolic - symbolic mapping task. The N1 amplitude increased across learning blocks in response to all symbols independent of their numerical range, but only in trials in which the symbols were preceded by dot-arrays representing a magnitude in the 1-4 subitizing range. Previous number processing ERP studies often attributed the presence of number distance effects on the N1 in non-symbolic tasks to perceptual differences in stimulus displays, such as size, contrast, or surface area (Gebuis and Reynvoet, 2013; Libertus et al., 2007; Soltész and Szucs, 2014). It is however unlikely that the current N1 training effects are due to such variations in visual stimulus characteristics because ERP responses were measured in response to the novel symbols that only show small perceptual differences. More importantly, the N1 amplitude change occurred across learning blocks that comprised the same symbolic stimuli and can thus not be due to perceptual stimulus variations between task blocks. If perceptual differences between the novel symbols would have been responsible for the $\mathrm{N} 1$ amplitude effect it should have been present already in the first learning session which was not the case; the N1 effect only emerged with learning. Instead, we interpret the increased N1 response to the novel symbols across learning blocks in only small dot array trials as a sign of perceptual surprise or expectancy violation, caused by a mismatch between the predicted and actual visual input. More specifically, we propose that only the small (1-4) dot arrays pre-attentively activated a specific magnitude code that, on different trials, created a perceptual mismatch with the magnitude code evoked by the novel symbol once it was learned in the last learning block. For such perceptual expectancy violations to occur one should have acquired one-to-one numerosity - symbol mappings, and in the current paradigm with short stimulus viewing times the development of such mappings was only possible for the dot-arrays 1-4 in the subitizing range for which numerosity is pre-attentively activated. Such an explanation is supported by recent studies by Johnston et al. (2017) and Robinson et al. (2018) in which 
an enhanced parieto-occipital N1/N170 response was found when viewing faces or objects that rotated in an unpredicted direction, which was interpreted as a sign of perceptual surprise or expectancy violation.

The second component of interest was the P2p. The literature has established in multiple paradigms that the $\mathrm{P} 2 \mathrm{p}$ component is responsive to experimental manipulations requiring access to numerical/magnitude codes. One such task that has been used most frequently is a number comparison task in which one has to decide whether two number stimuli represent the same or a different magnitude, decisions that can be only made when there is magnitude knowledge that has to be accessed in order to make such numerical decisions (Dehaene, 1996; Hyde and Spelke, 2009; Libertus et al., 2007; Rubinsten et al., 2013; Temple and Posner, 1998). On the basis of the assertion that the P2p reflects the processing stage at which one has first access to numerical representations (Dehaene, 1996; Hyde and Spelke, 2009; Libertus et al., 2007; Pinel et al., 2001; Temple and Posner, 1998), we hypothesized a modulation of the P2p with learning only for novel symbols that can be successfully mapped onto their corresponding numerosity, which we expected to be limited to the subitizing range (Benoit et al., 2004; Huang et al., 2010; Le Corre and Carey, 2007; Le Corre et al., 2006). In the learning stage in which the novel symbols have not yet gained any numerical meaning, or in stimulus conditions where exact symbol- quantity mapping is not possible (i.e. in the case of larger arrays of objects that can only be approximated when counting is not allowed), no such number information will/can be retrieved and there should thus be no P2p modulation. Indeed, a two-way interaction effect between training session and small/large symbol range showed a significant learning effect, with $\mathrm{P} 2 \mathrm{p}$ amplitude reducing from session 1 to session 3, only for symbols in the 1-4 numerical range and not for symbols in the 6-9 range. The current learning-related reduction of $\mathrm{P} 2 \mathrm{p}$ amplitude only for symbols 1-4 is congruent with the by now firmly established finding of smaller P2p amplitude in tasks with easy discriminable (e.g. spatially further apart) compared to harder discriminable number stimuli (e.g. the distance effect). Accordingly, the current $\mathrm{P} 2 \mathrm{p}$ amplitude reduction with learning can be interpreted as a sign of lower attentional effort needed to compare/discriminate the numerosities represented by small $(<5)$ dot-arrays and symbols once symbol- quantity associations increase in accuracy and strength due to learning. Finally, the finding that this symbol learning effect on the P2p for magnitudes in the subitizing range occurred independent of the numerical size of the preceding dot arrays (there were no dot-set size effects on the P2p), and occurred after correction for $\mathrm{N} 1$ effects, excludes the possibility that the P2p effects were influenced by changes in the visual properties (or priming effects) of preceding non-symbolic stimuli. 
An interesting question is whether the novel symbols representing numbers 1-4 were directly mapped onto their respective non-symbolic (quantity) representations or indirectly via number words. Since the current study population existed of college students with a fully developed symbolic number system and considerable exposure to complex mathematics, it is very well possible that the P2p effect reflects the mapping of the novel symbols onto number words that were automatically activated when seeing dot arrays in the subitizing range for which quick and exact magnitude estimates could be extracted. Related to this, it is assumable that there is a change in the nature of numerical representational systems across the life span. When first learning number symbols at preschool age, they might be mapped onto early existing, non-symbolic quantity representations, at least for the first four symbols (Carey, 2009). Later on, with more experience with and exposure to number symbols this may gradually shift to symbol - symbol (e.g. number word - digit) mapping and result in the development of a discrete semantic representational system as has recently been suggested in adults with an advanced symbolic number system (Krajcsi et al., 2016; Marinova et al., 2018; Reynvoet and Sasanguie, 2016).

There are as of yet few studies that have investigated symbol learning, especially the mapping of Arabic numerals on magnitude, in early childhood. While there is no consensus yet on the exact order of mapping, all studies agree that magnitude - spoken number word mapping takes place before magnitude - Arabic numeral mapping at respectively 3 and 4-5 years of age (Benoit et al., 2013; Hurst et al., 2016; Lira et al., 2017; Odic et al., 2015). More importantly for the present study, all these developmental studies report behavioral evidence for earlier and more accurate non-symbolic-symbolic mapping for small (1-3) than medium/large (4-9) numbers for which it is more difficult to determine cardinality, which is in line with the current mapping/learning task results in adults. A study by Sullivan \& Barner (2014), for example, concludes that children map words to magnitudes via associative mapping for the lower range (up until approximately six), but rely on an inferential process to acquire quantities above that. This is further expanded upon in a review by Carey \& Barner (Carey and Barner, 2019), in which it is proposed that learning of larger number words outside the subitizing range requires the knowledge and usage of counting algorithms and linguistic properties such as ordinality. Future studies might further investigate the electrophysiological correlates underlying the different stages of symbol number learning/mapping in early childhood. 
Concluding, the combined behavioral data from both our trial-and-error nonsymbolic - symbolic mapping/learning task and the number line placement task, suggest that adults only developed exact numeric representations for novel symbols representing a magnitude in the 1-4 (subitizing) range. This would be in agreement with suggestions that the foundation for symbolic number knowledge lies in the subitizing range, where exact numerical information can be quickly extracted from non-symbolic scenes and associated with words and symbols (Carey, 2009; Leibovich and Ansari, 2016; Reynvoet and Sasanguie, 2016; Siegler, 2016). Furthermore, only the P2p component showed non-symbolic - symbolic mapping related changes with learning that are indicative of gradually increased numerical access when the novel symbols acquired numerical meaning. The N1 amplitude did increase with learning for all symbols, but this effect was modulated by dot set-size in the sense that it only occurred on trials where symbols were preceded by dots in the 1-4 subitizing range. This symbolic N1 modulation on only small-dot trials was explained by expectancy violation that only occurred when one could quickly subitize an exact magnitude from the dot array that led to a prediction about the corresponding, learned, symbol, which subsequently caused a perceptual mismatch with the observed symbol. In contrast, the reduction of the P2p across learning blocks, interpreted as a decrease in effort to retrieve the numeric symbol code with learning, was not modulated by dot set-size and was only present for novel symbols in the low subitizing range, for which exact representations were acquired as shown by the behavioral data. These results support the conclusion that the P2p reflects the processing stage of first access to, or retrieval of, numeric codes and might in future studies thus be used as a neural correlate of symbol learning in the sense that it is only modulated when symbols acquire numerical meaning.

\section{Acknowledgements}

This study was partly supported by a grant from the Dutch Program Council for Educational Research from the Netherlands Organization of Scientific Research (NWO/PROO), grant number 411-07-112. The funders had no role in study design, data collection and analysis, decision to publish, or preparation of the manuscript. We thank Linda Essers for her contributions in the initial phase of designing the study and the programming of the tasks, and we thank Ruth Kessler for making and piloting the novel symbolic stimuli and for assistance in the data collection. 


\subsection{References}

Agrillo, C., Piffer, L., Bisazza, A., \& Butterworth, B. (2012). Evidence for two numerical systems that are similar in humans and guppies (S. F. Brosnan, Editor). PLoS ONE, 7(2), e31923. https://doi.org/10.1371/journal.pone.0031923 (cited on pages 68,69 )

Benoit, L., Lehalle, H., \& Jouen, F. (2004). Do young children acquire number words through subitizing or counting? Cognitive Development, 19(3), 291-307. https: //doi.org/10.1016/j.cogdev.2004.03.005 (cited on pages 84, 87)

Benoit, L., Lehalle, H., Molina, M., Tijus, C., \& Jouen, F. (2013). Young children's mapping between arrays, number words, and digits TT -. Cognition TA -, 129(1), 95-101 (cited on pages 69, 88).

Carey, S. (2009). The Origin of Concepts (Volume 133). Oxford University Press. https: //doi.org/10.1093/acprof:oso/9780195367638.001.0001. (Cited on pages 68-70, $88,89)$

Carey, S., \& Barner, D. (2019). Ontogenetic Origins of Human Integer Representations. https://doi.org/10.1016/j.tics.2019.07.004. (Cited on page 88)

Dehaene, S. (1992). Varieties of numerical abilities. Cognition, 44(1-2), 1-42. https : //doi.org/10.1016/0010-0277(92)90049-N (cited on page 68)

Dehaene, S. (1996). The Organization of Brain Activations in Number Comparison: Event-Related Potentials and the Additive-Factors Method. Journal of Cognitive Neuroscience, 8(1), 47-68. https://doi.org/10.1162/jocn.1996.8.1.47 (cited on pages $70,71,74,86,87$ )

Dehaene, S. (2001). Précis of The Number Sense. Mind \& language (Pages 16-36). https://doi.org/10.1111/1468-0017.00154. (Cited on pages 68, 69)

Dehaene, S., Dupoux, E., \& Mehler, J. (1990). Is numerical comparison digital? Analogical and symbolic effects in two-digit number comparison. Journal of Experimental Psychology: Human Perception and Performance, 16(3), 626-641. https://doi. org/10.1037/0096-1523.16.3.626 (cited on page 68)

Dehaene, S., Izard, V., \& Piazza, M. (2005). Control over non-numerical parameters in numerosity experiments. http://www.unicog.org/pm/pmwiki.php/Main/ Arithmetics (cited on page 74)

Diester, I., \& Nieder, A. (2007). Semantic associations between signs and numerical categories in the prefrontal cortex (S. Dehaene, Editor). PLoS Biology, 5(11), 2684-2695. https://doi.org/10.1371/journal.pbio.0050294 (cited on pages 70, 74) 
Diester, I., \& Nieder, A. (2010). Numerical values leave a semantic imprint on associated signs in monkeys. Journal of cognitive neuroscience, 22(1), 174-83. https://doi. org/10.1162/jocn.2009.21193 (cited on page 70)

Duncan, G. J., Dowsett, C. J., Claessens, A., Magnuson, K., Huston, A. C., Klebanov, P., Pagani, L. S., Feinstein, L., Engel, M., Brooks-Gunn, J., Sexton, H., Duckworth, K., \& Japel, C. (2007). School Readiness and Later Achievement. Developmental Psychology, 43(6), 1428-1446. https://doi.org/10.1037/0012-1649.43.6.1428 (cited on page 68)

Feigenson, L., Carey, S., \& Hauser, M. (2002). The representations underlying infants' choice of more: Object files versus analog magnitudes. Psychological Science, 13(2), 150-156. https://doi.org/10.1111/1467-9280.00427 (cited on page 69)

Feigenson, L., Dehaene, S., \& Spelke, E. (2004). Core systems of number. https://doi.org/ 10.1016/j.tics.2004.05.002. (Cited on page 69)

Gebuis, T., \& Reynvoet, B. (2013). The neural mechanisms underlying passive and active processing of numerosity. NeuroImage, 70, 301-307. https://doi.org/https : //doi.org/10.1016/j.neuroimage.2012.12.048 (cited on page 86)

Göbel, S. M., Watson, S. E., Lervåg, A., \& Hulme, C. (2014). Children's Arithmetic Development: It Is Number Knowledge, Not the Approximate Number Sense, That Counts. Psychological Science, 25(3), 789-798. https://doi.org/10.1177/ 0956797613516471 (cited on page 68)

Gross, H. J., Pahl, M., Si, A., Zhu, H., Tautz, J., \& Zhang, S. (2009). Number-based visual generalisation in the honeybee (H. Tanimoto, Editor). PLoS ONE, 4(1), e4263. https://doi.org/10.1371/journal.pone.0004263 (cited on page 68)

Halberda, J., \& Feigenson, L. (2008). Developmental Change in the Acuity of the "Number Sense": The Approximate Number System in 3-, 4-, 5-, and 6-Year-Olds and Adults. Developmental Psychology, 44(5), 1457-1465. https://doi.org/10.1037/ a0012682 (cited on page 69)

Holloway, I. D., \& Ansari, D. (2009). Mapping numerical magnitudes onto symbols: The numerical distance effect and individual differences in children's mathematics achievement. Journal of Experimental Child Psychology, 103(1), 17-29. https: //doi.org/10.1016/j.jecp.2008.04.001 (cited on page 68)

Huang, Y., Spelke, E. S., \& Snedeker, J. (2010). When is four far more than three? Children's generalization of newly-acquired number words. Psychological Science. 
http://journals . sagepub.com/doi/abs/10.1177/0956797610363552\%20http: //dash.harvard.edu/handle/1/4264760 (cited on pages 84, 87)

Hurst, M., Anderson, U., \& Cordes, S. (2016). Mapping among number words, numerals, and nonsymbolic quantities in preschoolers. Journal of Cognition and Development, 18(1), 41-62. https://doi.org/10.1080/15248372.2016.1228653 (cited on pages 69,88 )

Hyde, D. C., \& Spelke, E. S. (2009). All numbers are not equal: an electrophysiological investigation of small and large number representations. Journal of cognitive neuroscience, 21(6), 1039-1053. https://doi.org/10.1162/jocn.2009.21090 (cited on pages $70,71,86,87)$

Johnston, P., Robinson, J., Kokkinakis, A., Ridgeway, S., Simpson, M., Johnson, S., Kaufman, J., \& Young, A. W. (2017). Temporal and spatial localization of prediction-error signals in the visual brain. Biological Psychology, 125, 45-57. https://doi.org/10.1016/j.biopsycho.2017.02.004 (cited on page 86)

Krajcsi, A., Lengyel, G., \& Kojouharova, P. (2016). The source of the symbolic numerical distance and size effects. Frontiers in Psychology, 7, 1795. https://doi.org/10. 3389/fpsyg.2016.01795 (cited on page 88)

Le Corre, M., \& Carey, S. (2007). One, two, three, four, nothing more: An investigation of the conceptual sources of the verbal counting principles. Cognition, 105(2), 395-438. https://doi.org/10.1016/j.cognition.2006.10.005 (cited on pages 68, 84, 87)

Le Corre, M., Van de Walle, G., Brannon, E. M., \& Carey, S. (2006). Re-visiting the competence/performance debate in the acquisition of the counting principles. Cognitive Psychology, 52(2), 130-169. https://doi.org/10.1016/j.cogpsych.2005. 07.002 (cited on pages 84, 87)

Leibovich, T., \& Ansari, D. (2016). The Symbol-Grounding Problem in Numerical Cognition: A Review of Theory, Evidence, and Outstanding Questions. Canadian Journal of Experimental Psychology, 70(1), 12-23. https://doi.org/10.1037/cep0000070 (cited on pages 68, 69, 71, 89)

Libertus, M. E., Woldorff, M. G., \& Brannon, E. M. (2007). Electrophysiological evidence for notation independence in numerical processing. (Volume 3). https://doi.org/ 10.1186/1744-9081-3-1. (Cited on pages 70, 71, 74, 86, 87)

Lipton, J. S., \& Spelke, E. S. (2004). Discrimination of large and small numerosities by human infants. Infancy, 5(3), 271-290. https://doi.org/10.1207/s15327078in0503_2(cited on page 69) 
Lira, C. J., Carver, M., Douglas, H., \& LeFevre, J. A. (2017). The integration of symbolic and non-symbolic representations of exact quantity in preschool children. Cognition, 166, 382-397. https://doi.org/10.1016/j.cognition.2017.05.033 (cited on pages 69,88$)$

Lyons, I. M., Nuerk, H. C., \& Ansari, D. (2015). Rethinking the implications of numerical ratio effects for understanding the development of representational precision and numerical processing across formats. Journal of Experimental Psychology: General, 144(5), 1021-1035. https://doi.org/10.1037/xge0000094 (cited on page 71)

Marinova, M., Sasanguie, D., \& Reynvoet, B. (2018). Symbolic estrangement or symbolic integration of numerals with quantities: Methodological pitfalls and a possible solution (S. J. Cropper, Editor). PLoS ONE, 13(7), e0200808. https://doi.org/10. 1371/journal.pone.0200808 (cited on page 88)

McCandliss, Posner, \& Givon. (1997). Brain Plasticity in Learning Visual Words. Cognitive Psychology, 33(1), 88-110. https://www.sciencedirect.com/science/article/pii/ S0010028597906617 (cited on page 73)

Merkley, R., Shimi, A., \& Scerif, G. (2016). Electrophysiological markers of newly acquired symbolic numerical representations: the role of magnitude and ordinal information. ZDM - Mathematics Education, 48(3), 279-289. https://doi.org/10. 1007/s11858-015-0751-y (cited on pages 71, 72)

Moyer, R. S., \& Landauer, T. K. (1967). Time required for judgements of numerical inequality. Nature, 215(5109), 1519-1520. https://doi.org/10.1038/2151519a0 (cited on page 68)

Nieder, A., \& Dehaene, S. (2009). Representation of Number in the Brain. Annual review of neuroscience, 32(1), 185-208. https://doi.org/10.1146/annurev.neuro.051508. 135550 (cited on page 69)

Nieder, A., Diester, I., \& Tudusciuc, O. (2006). Temporal and spatial enumeration processes in the primate parietal cortex. Neuroforum, 12(4), 267-269. https://doi.org/10. 1126/science.1130308 (cited on page 70)

Odic, D., Le Corre, M., \& Halberda, J. (2015). Children's mappings between number words and the approximate number system. Cognition, 138, 102-121. https: //doi.org/10.1016/j.cognition.2015.01.008 (cited on page 88)

Park, J., Chiang, C., Brannon, E. M., \& Woldorff, M. G. (2014). Experience-dependent hemispheric specialization of letters and numbers is revealed in early visual 
processing. Journal of cognitive neuroscience, 26(10), 2239-2249. https://doi. org/10.1162/jocn_a_00621 (cited on page 70)

Piazza, M. (2010). Neurocognitive start-up tools for symbolic number representations. Trends in Cognitive Science, 14(12), 542-551. https://doi.org/10.1016/j.tics.2010. 09.008 (cited on pages 68, 69)

Piazza, M., Fumarola, A., Chinello, A., \& Melcher, D. (2011). Subitizing reflects visuospatial object individuation capacity. Cognition, 121(1), 147-153. https://doi.org/ 10.1016/j.cognition.2011.05.007 (cited on page 69)

Pinel, P., Dehaene, S., Rivière, D., \& LeBihan, D. (2001). Modulation of parietal activation by semantic distance in a number comparison task. NeuroImage, 14(5), 1013-1026. https://doi.org/10.1006/nimg.2001.0913 (cited on pages 70, 71, 87)

Revkin, S. K., Piazza, M., Izard, V., Cohen, L., \& Dehaene, S. (2008). Does subitizing reflect numerical estimation? Psychological Science, 19(6), 607-614. https://doi. org/10.1111/j.1467-9280.2008.02130.x (cited on page 69)

Reynvoet, B., \& Sasanguie, D. (2016). The symbol grounding problem revisited: A thorough evaluation of the ans mapping account and the proposal of an alternative account based on symbol-symbol associations. https://doi.org/10.3389/fpsyg.2016.01581. (Cited on pages 68-70, 88, 89)

Robinson, J. E., Breakspear, M., Young, A. W., \& Johnston, P. J. (2018). Dose-dependent modulation of the visually evoked N1/N170 by perceptual surprise: A clear demonstration of prediction-error signalling. https://doi.org/10.1111/ejn.13920. (Cited on page 86)

Rubinsten, O., Dana, S., Lavro, D., \& Berger, A. (2013). Processing ordinality and quantity: ERP evidence of separate mechanisms. Brain and Cognition, 82(2), 201-212. https://doi.org/https://doi.org/10.1016/j.bandc.2013.04.008 (cited on pages 70, $71,86,87)$

Siegler, R. S. (2016). Magnitude knowledge: The common core of numerical development. Developmental Science, 19(3), 341-361. https://doi.org/10.1111/desc.12395 (cited on pages $68-70,89$ )

Siegler, R. S., \& Ramani, G. B. (2009). Playing Linear Number Board Games-But Not Circular Ones-Improves Low-Income Preschoolers' Numerical Understanding. Journal of Educational Psychology, 101(3), 545-560. https://doi.org/10.1037/ a0014239 (cited on page 68) 
Soltész, F., \& Szucs, D. (2014). Neural adaptation to non-symbolic number and visual shape: An electrophysiological study. Biological Psychology, 103, 203-211. https://doi.org/10.1016/j.biopsycho.2014.09.006 (cited on pages 70, 86)

Spelke, E. S. (2017). Core Knowledge, Language, and Number. Language Learning and Development, 13(2), 147-170. https://doi.org/10.1080/15475441.2016.1263572 (cited on page 68)

Sullivan, J., \& Barner, D. (2012). How are number words mapped to approximate magnitudes? Quarterly Journal of Experimental Psychology, 66(2), 389-402. https://doi.org/10.1080/17470218.2012.715655 (cited on page 68)

Sullivan, J., \& Barner, D. (2014). Inference and association in children's early numerical estimation. Child Development, 85(4), 1740-1755. https://doi.org/10.1111/cdev. 12211 (cited on pages 69, 88)

Temple, E., \& Posner, M. I. (1998). Brain mechanisms of quantity are similar in 5-year-old children and adults. Proceedings of the National Academy of Sciences of the United States of America, 95(13), 7836-41. https://doi.org/10.1073/pnas.95.13. 7836 (cited on pages 70, 71, 74, 86, 87)

Trick, L. M., \& Pylyshyn, Z. W. (1994). Why are small and large numbers enumerated differently? A limited-capacity preattentive stage in vision. Psychological Review, 101(1), 80-102. https://doi.org/10.1037/0033-295X.101.1.80 (cited on page 69)

Turconi, E., Jemel, B., Rossion, B., \& Seron, X. (2004). Electrophysiological evidence for differential processing of numerical quantity and order in humans. Cognitive Brain Research, 21(1), 22-38. https://doi.org/10.1016/j.cogbrainres.2004.05.003 (cited on page 70)

Wynn, K. (1990). Children's understanding of counting. Cognition, 36(2), 155-193. https: //doi.org/10.1016/0010-0277(90)90003-3 (cited on page 68)

Wynn, K. (1992). Children's acquisition of the number words and the counting system. Cognitive Psychology, 24(2), 220-251. https://doi.org/10.1016/0010-0285(92) 90008-P (cited on page 68)

Xia, L., Siemann, M., \& Delius, J. D. (2000). Matching of numerical symbols with number of responses by pigeons. Animal Cognition, 3(1), 35-43. https://doi.org/10.1007/ s100710050048 (cited on pages 68, 69)

Xu, F., \& Spelke, E. S. (2000). Large number discrimination in 6-month-old infants. Cognition, 74(1), B1-B11. https://doi.org/10.1016/S0010-0277(99)00066-9 (cited on page 69) 
Zhao, H., Chen, C., Zhang, H., Zhou, X., Mei, L., Chen, C., Chen, L., Cao, Z., \& Dong, Q. (2012). Is Order the Defining Feature of Magnitude Representation? An ERP Study on Learning Numerical Magnitude and Spatial Order of Artificial Symbols (E. Akyürek, Editor). PLoS ONE, 7(11). https://doi.org/10.1371/journal.pone. 0049565 (cited on pages 71, 72) 

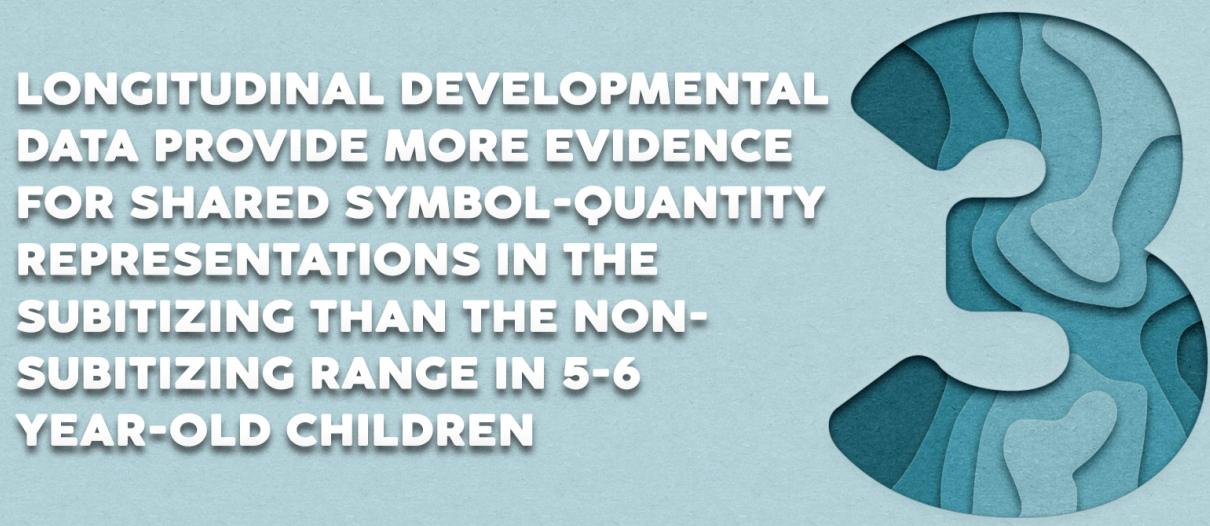

\subsection{Abstract}

One influential theory on number development is the ANS mapping account, which proposes that the acquisition and discrimination of symbolic numbers (i.e., number words and digits) is supported by early present representations of magnitude in the Approximate Number System (ANS). In the current developmental study, we use a mixed longitudinal-cross-sectional design in combination with measurement of Event-Related Potentials (ERPs) to investigate this claim. Whereas children and adults both showed behavioral distance effects, neural P2p distance effects were present for both numerical ranges in adults but only for the subitizing range in children. Under the assumption that the P2p reflects access to the analog quantity representation that the symbol is associated with, these results imply that children relied on these associations for the subitizing range but not the non-subitizing range. Thus, these combined $\mathrm{P} 2 \mathrm{p}$ and behavioral findings support exact non-symbolic - symbolic mapping underlying the learning of number symbols in the subitizing range, but not the non-subitizing range. According to the $\mathrm{P} 2 \mathrm{p}$ distance data, development diverges for the later 6-9 number range. The mapping between symbolic and non-symbolic representations is present in adults, suggesting that these associations are made eventually, through different processes than direct mapping.

R Corresponding reference: van den Berg, F. C. G. \& Jonkman, L. M. (in prep). Longitudinal electrophysiological data provide more evidence for shared number symbol-quantity representations in the subitizing than the nonsubitizing range in 5-6-year-old children. 


\subsection{Introduction}

Humans have different numerical systems to process and represent quantities; one is the Approximate Number System (ANS; Feigenson et al., 2004) involved with the processing of quantities in a non-symbolic form (e.g., numbers of objects such as three apples). Another, the Symbolic Number System (SNS), regulates the processing of number symbols (number words like 'three' and Arabic numerals like '3'). ANS processing abilities have been shown in preverbal infants (Di Giorgio et al., 2019; Xu and Spelke, 2000; Xu et al., 2005) and in animals (Geary et al., 2015; Leibovich et al., 2017). Number symbols, on the other hand, have to gain numerical meaning through formal education. How number symbols acquire their numerical meaning is still debated and largely unknown (Reynvoet and Sasanguie, 2016; Wilkey and Ansari, 2019). The seemingly innate nature of ANS abilities led some researchers to propose the ANS mapping account (Dehaene, 2001; Piazza, 2010) which assumes that number symbols acquire numerical meaning via direct mapping onto their corresponding non-symbolic representations of quantity in the ANS. However, in recent years the ANS Mapping account has been questioned (for reviews see: Leibovich and Ansari, 2016; Noël and Rousselle, 2011; Reynvoet and Sasanguie, 2016), and some have suggested that direct non-symbolic to symbolic mapping might occur only for small quantities 1-4, which can be easily enumerated and thus exactly represented using the Object Tracking System (OTS; Carey, 2009a; Carey and Barner, 2019). The present developmental study aims to add to the existing literature by addressing whether only small number symbols representing a magnitude in the 1-4 subitizing range are directly mapped onto/associated with non-symbolic representations of quantity (as opposed to higher symbols 6-9). As explained more elaborately below, we will address this question using a mixed longitudinal/cross-sectional study design, investigating the developmental stability or change in numerical distance effects on performance and the P2p event-related brain potential component across non-symbolic and symbolic number comparison tasks for numbers in the subitizing (1-4) versus the non-subitizing (6-9) range.

The precision of, and overlap between, non-symbolic and symbolic number representations has historically been assessed using number comparison tasks. In such tasks, participants must decide which of two stimuli (non-symbolic, symbolic, or a mix of both) represents a larger or smaller quantity. The main outcome measure indicative of the development or acuity of number representations in number comparison tasks is the Number Distance Effect (NDE; Moyer and Landauer, 1967) for symbolic tasks or the Number Ratio Effect (NRE; Buckley and Gillman, 1974) for non-symbolic tasks. The 
NDE/NRE reflect faster and more accurate responses as the numerical distance/ratio between to-be-compared number symbols/quantities increases. Dehaene (1992, 2001; 1990) suggested that NDE/NRE's result from the way numbers are represented on a mental number line, with numerically closer numbers or quantities having more representational overlap than those further apart. The higher the representational overlap between two numbers/quantities, the more attention/effort is required for their discrimination, which is reflected by lower accuracy and slower response times (i.e., the NDE). In number comparison tasks comprising symbolic number stimuli (e.g., number words or Arabic digits) that must gain numeric meaning through learning, an NDE will only be present once symbols have gained numerical meaning. In other words, symbols can only show an NDE when they have been successfully associated with the quantity they represent in the outside world. The similarities between NDE's in symbolic, and NRE's in non-symbolic number comparison tasks in adults (Dehaene, 2011) has led to the suggestion that they share representations (both being represented in an analog way in the ANS). Some researchers (Dehaene, 2001; Piazza, 2010) concluded that the later acquired number symbols were directly mapped onto their corresponding, earlier present, non-symbolic representations of magnitude in the ANS.

Multiple developmental studies in children have reported similar NDE's on reaction time or accuracy in non-symbolic and symbolic number comparison tasks (Barth et al., 2005; Halberda and Feigenson, 2008; Sekuler and Mierkiewicz, 1977). Further, similar decreases in the size of the NDE in non-symbolic and symbolic comparison tasks have been reported with increasing age (Duncan and McFarland, 1980; Halberda and Feigenson, 2008; Holloway and Ansari, 2009; Sasanguie et al., 2012; Sekuler and Mierkiewicz, 1977), pointing to an improved ability to discriminate quantities/number symbols across childhood, due to the refinement of number representational systems (less representational overlap between numbers nearby on the mental number line) when children start developing more number expertise in elementary school. The similar NDEs and comparable developmental patterns of NDEs in children across symbolic and non-symbolic tasks were first taken as additional evidence in favor of shared representational systems and thus the ANS mapping account (Dehaene, 2001). However, as noted by Wilkey and Ansari (2019), most of these studies did not use longitudinal designs and were thus not able to study within-individual changes between symbolic and non-symbolic number processing (using NRE or NDE effects) across developmental time. Further, the few studies that did use a longitudinal study design only found unidirectional relations, with symbolic NDEs predicting the development of non-symbolic NDEs but not the other way around (Lyons 
et al., 2018; Matejko and Ansari, 2016; Mussolin et al., 2014; Sasanguie et al., 2014). Such a unidirectional relationship argues against symbols being directly mapped onto non-symbolic representations of number in the ANS. Another argument against shared symbolic - non-symbolic representations comes from ample evidence that children's NDEs in symbolic number comparison tasks are stronger predictors of children's later symbolic/math skills than non-symbolic NDE's (Habermann et al., 2020; Schneider et al., 2017; Xenidou-Dervou et al., 2017), which is not expected if both rely on the same representational system.

Other more recent findings in adults also challenge the ANS mapping account (for elaborate reviews see: Carey and Barner, 2019; Leibovich and Ansari, 2016; Reynvoet and Sasanguie, 2016; Wilkey and Ansari, 2019). For example, two behavioral studies showed that adults responded slower when they had to make quantity comparisons across symbolic and non-symbolic number notations (e.g., words/numerals versus dot arrays), compared to making within-notation (symbol-symbol) comparisons (Lyons et al., 2012; Marinova et al., 2018, 2021). Lyons et al., (2012) argued that these additional processing costs during cross-notation comparisons reflected non-shared representational systems and used the term 'symbolic estrangement,' proposing that due to more experience with, and exposure to, Arabic digits during life, other representational systems and links develop and number symbols get more and more estranged from their non-symbolic counterparts (at least in adults). A recent cross-sectional developmental study by Marinova \& Reynvoet, (Marinova and Reynvoet, 2020) reported higher overall accuracy in symbol-symbol (word/digit) comparison tasks than in cross-notation (symbolic -non-symbolic) or mixed auditory/visual non-symbolic tasks in third and fifth graders. The authors interpreted these results as evidence for symbolic estrangement at this age. Finally, results from fMRI studies in adults that compared brain activation patterns during the performance of non-symbolic and symbolic tasks also found no evidence for shared underlying neural substrates (see for review: Wilkey and Ansari, 2019).

The above-reviewed studies in children and adults do not support the existence of a shared analog representational system for symbolic and non-symbolic number stimuli and argue against the ANS mapping account. However, these studies did not consider symbolic - non-symbolic mapping differences across age between subitizable and nonsubitizable quantities and have mostly excluded stimuli in the subitizing range. Whereas in the Symbolic Number System, any quantity (small or large) is represented by only one symbol and thus with exact precision, this is not the case in the ANS. In the ANS, an exact 
value can only be assigned to small quantities 1-4 within the subitizing range that can be enumerated accurately at a glance, without the need for time- and effort-consuming counting processes (Kaufman et al., 1949; Mandler and Shebo, 1982). Non-symbolic quantities outside the subitizing range can only be enumerated exactly by counting, but when there is no time or one lacks the mental resources to count, the size of larger quantities can only be estimated, yielding an approximate value. Accordingly, direct symbolic non-symbolic mapping has been suggested to be more likely for quantities in the subitizing range for which an exact cardinality value can be quickly extracted (Carey, 2009b; Carey and Barner, 2019; Feigenson et al., 2002; Feigenson et al., 2004; Uller et al., 1999).

To the best of our knowledge, only one longitudinal developmental study has compared non-symbolic and symbolic processing overlap within and outside the subitizing range (Hutchison et al., 2019). In this study, symbolic and non-symbolic number comparison tasks were administered to 540 five-year-old kindergartners at two time-points six months apart to study the uni- and bi-directionality of symbolic and non-symbolic processing accuracy across developmental time within the subitizing (1-4) and non-subitizing range (6-9). A bidirectional relation between symbolic and non-symbolic processing was found within the subitizing range at both measurement points. However, the non-subitizing range displayed a unidirectional relationship, with symbolic processing at time point 1 predicting non-symbolic processing at time point 2 , but not vice versa. These data support the view that, at least in five-year-old children, only symbols and quantities in the subitizing range are directly mapped onto each other whereas digits in the non-subitizing range are not. These results are in line with several behavioral studies in preschoolers demonstrating better symbolic - non-symbolic mapping for quantities in the subitizing range for which one can easily derive the cardinality (Benoit et al., 2013; Hurst et al., 2016; Lira et al., 2017; Sullivan and Barner, 2014). Hutchison et al's data further suggest that in five-year-old children, the development of Arabic digit knowledge influences the development of non-symbolic knowledge instead of the other way around as suggested by the ANS mapping account.

The above discussed longitudinal study by Hutchison did however include a more general comparison accuracy measure (the number of correct comparisons made within a 2-minute time-limit) and did not study the development of number distance or ratio effects that are assumed to be a signature of magnitude (ANS) processing. Further, it has been suggested that distance effects on performance measures like reaction time or accuracy in comparison tasks with number symbols (words/digits) can also originate from other, non- 
magnitude related, processes (Cohen, 2009; Cohen et al., 2013; Krajcsi and Kojouharova, 2017; Krajcsi et al., 2016a; Lin and Göbel, 2019; Reynvoet and Sasanguie, 2016). In contrast to behavioral measures such as response times and accuracy that are outcome measures, Event-Related Potentials (ERP) reflect the perceptual-semantic processing of stimuli at millisecond level and can be used to elucidate the processes underlying behavioral numerical distance effects. One ERP component commonly accepted as reflecting early (first) access to analog representations of magnitude is the P2p (Dehaene, 1996), which is the second positive ERP component, in adults appearing around 200 milliseconds after stimulus presentation above lateral or right-hemisphere parietal-occipital electrode sites (Dehaene, 1996; Hyde and Spelke, 2012; Libertus et al., 2007; Temple and Posner, 1998). Dehaene (1996) was the first to report that in a healthy adult sample, P2p amplitude increased with decreasing numerical distance between a presented Arabic digit (range 1-9) and the internal reference number five while making magnitude comparisons (e.g., is the shown Arabic digit smaller or larger than five). This increase in P2p amplitude with decreasing numerical distance signals the recruitment of more cognitive resources to discriminate two stimuli that are numerically closer together on the mental number line and are consequently more difficult to discriminate. Dehaene (1996) accordingly concluded that $\mathrm{P} 2 \mathrm{p}$ represents the processing stage in which one accesses the semantic meaning (via analog magnitude representations in the ANS) associated with the Arabic digits. Since Dehaene's first study, several studies in adults have replicated these effects of numerical distance on P2p amplitude in response to Arabic digits 1-9 in magnitude comparison tasks (Hyde and Spelke, 2009, 2012; Libertus et al., 2007; Pinel et al., 2001), but also in non-symbolic number comparison tasks with higher magnitudes (Rubinsten et al., 2013). In a recent study, we presented adults with a trial-and-error learning (same/different) task with feedback in which novel abstract symbols had to acquire numerical meaning by mapping them onto quantities 1-9 (van den Berg et al., 2020). The combined behavioral and $\mathrm{P} 2 \mathrm{p}$ results provided evidence for successful (exact) symbol-quantity mapping only taking place for subitizable quantities 1-4 and not for quantities 6-9 for which (due to the short presentation time) no exact quantity could be derived without counting. P2p amplitude decreased across learning blocks, which was interpreted as a decrease in the effort to retrieve the semantic (numeric) information associated with the symbol across learning blocks. This P2p amplitude decrease with learning was however only present for novel symbols in the subitizing (1-4) range, for which exact numeric representations were acquired as shown by the behavioral data. 
These results support the conclusion that the $\mathrm{P} 2 \mathrm{p}$ reflects the processing stage of first access to or retrieval of numeric (magnitude) codes.

There are to our knowledge only a few and only cross-sectional ERP studies that have investigated magnitude processing in symbolic and/or non-symbolic number comparison tasks and their neural correlates in children (Gómez-Velázquez et al., 2015; Gouet et al., 2018; Heine et al., 2011; Heine et al., 2013; Temple and Posner, 1998). These studies have found similar numerical distance effects (NDEs) on P2p amplitude in children as were previously found in adults in symbolic and non-symbolic number comparison tasks. Only two of these studies investigated distance effects on children's performance and ERPs separately for non-symbolic stimuli (arrays of dots) in the subitizing and non-subitizing (counting and estimation) ranges (Heine et al., 2011; Heine et al., 2013). In Heine et al. (2013) non-symbolic number distance effects on performance and posterior ERP amplitude in a 280-600ms post-stimulus time window were found in 8-year-old children with and without Mathematical Learning Difficulties (MLD). Although children with MLD generally performed worse, both groups showed similar distance effects on performance that did not differ between small-subitizable and larger-nonsubitizable quantities in the counting or estimation range. In contrast to the behavioral results, distance effects on the amplitude of the posterior positive ERP component did differ between the groups; whereas children without MLD showed similar distance effects on posterior ERP amplitude for subitizable and non-subitizable quantities, children with MLD only showed a distance effect on posterior ERP amplitude for quantities in the subitizing range. The authors concluded that MLD does not affect automatic subitizing processes but does affect estimation processes needed for the comparison of higher quantities.

The above-reviewed studies thus indicate that numerical distance effects on the P2p-ERP component can be reliably measured in children and can provide additional information above performance measures about the semantic (numeric) processing of Arabic digits and quantities and their mapping in subitizing and non-subitizing ranges. In the present study developmental change or stability in numerical distance effects on the ERP-P2p component and performance in symbolic (Arabic digits) and non-symbolic (dot arrays) number comparison tasks and subitizing (1-4) and non-subitizing (6-9) ranges will be studied across two longitudinal time points (e.g., in the last year of kindergarten and approximately 10 months later in first grade). 
This particular age range was chosen because children start to learn about Arabic digits in this time period and developmental shifts with regard to Arabic digit knowledge likely occur in the 4-5 preschool period and the 6-7 school entry period (Knudsen et al., 2015).

Based on the exact mapping hypothesis (Carey, 2009b; Carey and Barner, 2019) and the above reviewed findings we hypothesize that in children exact, one-to-one Arabic digit - quantity mapping will have only occurred between symbols and quantities in the subitizing range. As explained above, based on the prior number processing ERP literature, recruitment of shared magnitude representations in symbolic/non-symbolic comparison tasks should become visible as similar numerical distance effects on P2p amplitude and behavioral measures (reaction time and accuracy) across development for digits/quantities 1-4. In the here studied age range no developmental change in numerical distance effects on reaction time or $\mathrm{P} 2 \mathrm{p}$ amplitude is expected for digits/dot arrays 1-4 because digit-quantity mapping is expected to be completed for this range by the age of five years (Knudsen et al., 2015). Based on suggestions from the developmental number processing literature that during acquisition of number symbols 6-9 outside the subitizing range, children rely more on other information like the ordinality of number sequences and symbol-symbol associations than on one-to-one digit- quantity mapping (Carey, 2009b; Carey and Barner, 2019; Marinova and Reynvoet, 2020), we expect symbolic-P2p distance effects to be weaker or absent in the non-subitizing range in children.

\subsection{Method}

\subsubsection{Participants}

Participants were kindergarten children who took part in the large-scale longitudinal project MathChild. The present behavioral and ERP data were gathered in only a small subset of the children who participated in a larger behavioral number processing study (MathChild project). In the present study, different tasks than in the larger project were used, and separate ethical approval and written informed consent from parents were obtained. The participating children attended one of eight different primary schools in the South of the Netherlands, and parents were informed about the study via distribution of information packages containing an information letter explaining all study specifics accompanied by an informed consent form. 
The initial sample consisted of 39 children (at the first measurement the age of the children ranged between range 5yrs $6 \mathrm{mo}$ and 6yrs $6 \mathrm{mo}$ : mean age: 6 years [SD 3mo]; 20 boys). Only data from 26 children from whom complete behavioral and EEG data sets were acquired in the last year of kindergarten as well as one year later in grade 1 were included in the present analyses. Of the thirteen excluded children, five did not participate in the second measurement, seven did not tolerate the EEG measurement, and one child scored far below chance-level on all tasks. The age of the remaining 26 children (of which 12 were boys) at the first measurement point in kindergarten was 5yrs and 11 mo (age range $5 \mathrm{yrs} 6 \mathrm{mo}-6 \mathrm{yrs} 5 \mathrm{mo}$; SD 3mo), and at the second measurement in grade 1 this was 6yrs $9 \mathrm{mo}$ (age range 6yrs $5 \mathrm{mo}-7 \mathrm{yrs} 4 \mathrm{mo}$, SD: 3mo). The children were rewarded with a small present after each EEG testing session. Adult data was collected from 22 participants recruited from the student population at Maastricht University who participated in a larger number learning study of which data are published in another paper (van den Berg et al., 2020).

The data included in the present study concerns only the two standard non-symbolic and symbolic number comparison tasks that were performed completely at the beginning of the learning sessions of the larger study. The data from these tasks were not included in the earlier paper and have not been published before. One adult participant reported a headache due to wearing the EEG cap and did, therefore, not complete the tasks; one other adult was excluded because of below chance performance on the non-symbolic comparison task. All reported analyses included the remaining 20 participants (mean age: $22 \mathrm{yrs} 2 \mathrm{mo}$ [SD 3yrs 10mo]; 2 males). All adults provided written informed consent after being informed about the study. Participants were rewarded with $7.50 €$ per hour in the form of VVV vouchers or one course credit per hour. Both studies were approved by a local Ethical Committee of the Faculty.

\subsubsection{Procedure}

All children underwent a total of two testing sessions, separated by approximately one academic year. Testing took place at the children's school and started with an explanation of the procedure, followed by attachment of the electrodes, during which children watched cartoons. Thereafter all children performed the non-symbolic and a symbolic number comparison tasks (for explanation of the tasks see 2.3.) while their EEG was measured. The order of task presentation was counterbalanced between the children within a measurement session, and task order was reversed for each child between the two measurements. The children were instructed to respond as fast and as accurately as possible and were asked to 
minimize eye blinks and (eye) movements during the tasks. Before testing, the children practiced the upcoming task in blocks of six practice trials until a performance criterion of $80 \%$ correct was reached.

After the explanation of the procedure and signing the informed consent form, all adults performed the same non-symbolic and symbolic number comparison tasks in a lab at the University. The adults received the same instructions concerning speed and accuracy as the children, they performed 15 practice trials, and the order of task presentation was counterbalanced between participants.

\subsubsection{Number Comparison Task}

The current symbolic (Arabic digits) and non-symbolic (dot stimuli) tasks were so-called number comparison-to-referent tasks (Dehaene et al., 1996; Libertus et al., 2007; Maloney et al., 2010; Temple and Posner, 1998). Both tasks included a small number range (1, 2, 3, and 4) and a larger number range (6, 7, 8, and 9). All stimuli were presented at the center of the screen, and participants were required to compare each number stimulus with the mental reference number five and to decide whether the number stimulus was smaller or larger than five. Responses were recorded via a button box; a left button had to be pressed when the dot/digit stimuli were smaller than five, a right button when presented dots/digits were larger than five.

In the children study, each task comprised 160 trials (20 trials per number; 5 blocks of 32 trials), whereas, in the adult study, there were 240 trials per task ( 30 trials per number; 2 blocks of 120 trials). Stimulus presentation was quasi-random to prevent identical numbers from being shown twice in a row; breaks were given between each block. See Figure 1 for an example of a trial in the comparison tasks for children, including information about stimulus presentation, inter-trial, and response time windows. Stimuli were presented in white on a black background at the center of a 17-inch monitor (children) or black on grey on a 19-inch monitor (adults) at a viewing distance of $57 \mathrm{~cm}$. The Arabic digits were presented in font Times New Roman font size 200 and were $8.6 \mathrm{~cm}$ in height. In the non-symbolic task, patterns of dots were presented within an invisible circle with a diameter of $11.7 \mathrm{~cm}$. Differences in dot size and area were controlled for by fixing the dot-diameter in one half of the trials and dot-size in the other, as suggested by Dehaene, Izard, and Piazza (2005). Furthermore, to prevent responses from getting linked to specific dot patterns instead of dot quantity, four dot pattern variations were presented for each quantity. 


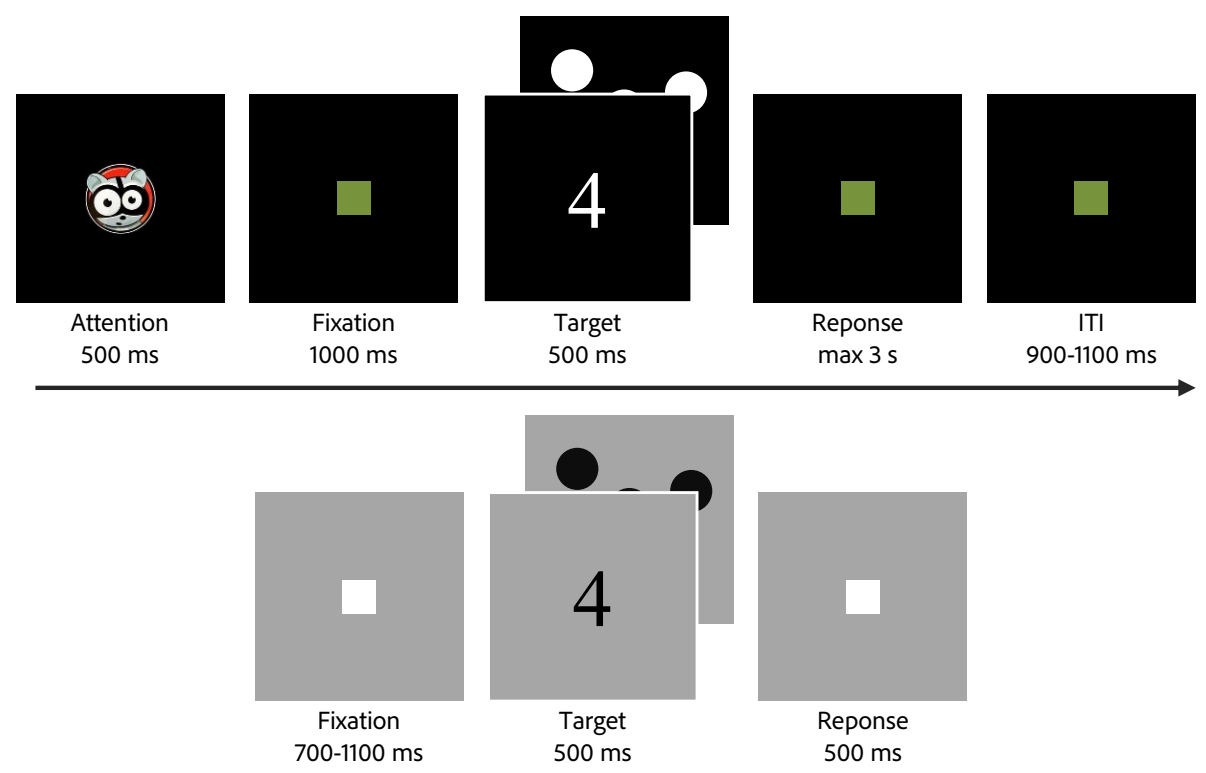

Figure 3.1: example of a trial for the symbolic and non-symbolic comparison tasks for children (top) and adults (bottom)

\subsubsection{EEG/ERP Acquisition and Analyses}

Electro-Encephalographic (EEG) measurements took place in a quiet environment at the schools of the children (i.e., no disturbing sounds or electronic devices), and adults underwent the EEG measurements in a lab at the University. For children, each of the two testing sessions lasted 2.5-3 hours, starting with the attachment of an elastic EEG-electrode cap (EasyCap; Nellcor-Puritan Bennet, Hayward, CA) with a 38 tin electrode set-up (AF7, AF8, AFz, C3, C4, CP1, CP2, CP5, CP6, Cz, F3, F4, F5, F6, F7, F8, FC1, FC2, FC5, FC6, FCz, FP1, FP2, Fz, O1, O2, Oz, P3, P4, P5, P6, P7, P8, PO7, PO8, Pz, T7, T8). In adults, EEG data were recorded using a 64-channel electrode cap (EasyCap: $\mathrm{Ag} / \mathrm{AgCl}$ ).

The data were filtered online at $0.01-225 \mathrm{~Hz}$ and continuously sampled at a rate of $500 \mathrm{~Hz}$ using a BrainAmp amplifier system and software (Brain Products GmbH, Munich, Germany). The POz electrode served as ground, and the left mastoid (A1) acted as the online reference; the right mastoid (A2) was included as an extra active electrode. Horizontal and vertical EOG was measured by electrodes placed on respectively the outer canthus of each eye and above/below the left orbit. All electrode impedances were kept below 5-10 kOhm and were frequently checked during testing. 
For offline analyses of the EEG data, Matlab2019a/EEGlab2019/ERPLABv7.0.0 software was used. The data was first resampled to $250 \mathrm{~Hz}$ and re-referenced to the average signal. Next, a band-pass filter of $0.1 \mathrm{~Hz}$ to $70 \mathrm{~Hz}$ was applied to the data before execution of Independent Component Analysis (ICA) for removal of horizontal eye movements and blinks. The ocular artifact-free data was filtered using a $30 \mathrm{~Hz} \mathrm{Low}$-pass filter, after which the data was epoched (based on the dot-quantity and symbol-quantity event-codes, excluding epochs with incorrect responses) into $200 \mathrm{~ms}$ pre-stimulus and $500 \mathrm{~ms}$ poststimulus windows. Baseline correction was performed using the $200 \mathrm{~ms}$ pre-stimulus interval. Next, an automatic artifact detection procedure was applied to the remaining epochs (only at relevant electrodes: PO7 and PO8 for children and PO3/PO4 and P5/P6 for adults), rejecting trials with activity exceeding a $\pm 100 \mu \mathrm{V}$ threshold in children and $\pm 75 \mu \mathrm{V}$ for adults. A higher criterion was adopted for children because of higher EEG amplitudes. In the child data from the symbolic number comparison task, an average of $20.20 \%(18.50 \%$ [2.75-37.25\%] due to incorrect responses, and 2.10\% [0-16.47\%] due to artifacts) of the total number of trials were rejected in session 1 and $13.06 \%$ in session 2 (of which $12.26 \%$ [1.50-34.50\%] due to incorrect responses and 0.89\% [0-10.45\%] due to artifacts).In the non-symbolic child data respectively $23.28 \%$ (21.90\% [2.50-43.50\%] incorrect responses; $1.87 \%$ [0-11.03\%] artifacts) and $15.72 \%$ (15.03\% [0-37.75\%] incorrect responses; $0.85 \%$ [0-4.87\%] artifacts) of total trials was rejected in sessions 1 and 2 . For the adult data the average trial rejection rate was $5.24 \%(4.90 \%$ [1.67-9.85\%] incorrect trials; $0.35 \%$ [0-1.75\%] artifacts) in the symbolic task and $11.25 \%$ (10.93\% [2.50-42.08\%] incorrect responses; $0.34 \%$ [0-2.66\%] artifacts) in the non-symbolic task. Participants were only included in the subsequent analyses if at least $50 \%$ of total trials in each range-distance category remained for averaging (after removal of incorrectly responded and artifact trials).

The choice of electrodes and time-windows to be included in the statistical analyses was based on the previous number-processing ERP literature using the same comparisonto-referent tasks with dots/digits 1-9 (Dehaene, 1996; Libertus et al., 2007; Pinel et al., 2001; Temple and Posner, 1998) and on visual inspection of the grand average ERP signals and topography maps in the different conditions and the different age groups. Based on this, in children, mean P2p amplitude was computed in a 300-360 ms time window after stimulus presentation at PO7 and PO8 electrodes in both tasks (see Figure 3 for Grand Average ERPs and topo-maps). In adults, in the symbolic task mean P2p amplitude was computed in a 210-280 ms time window at PO3/PO4 electrodes and in the non-symbolic task in a 230-290 ms time window at P5/P6 electrodes. Mean P2p amplitude values within these time windows were entered in the statistical analyses. 


\subsubsection{Statistical Analysis}

For dependent measures RT and P2p amplitude, separate Linear Mixed-effects models were constructed for the Symbolic and Non-symbolic tasks. For the accuracy data, Generalized Estimation Equation (GEE) models were constructed because of severe violation of normality. All models included within-subject factors Numerical Range (2 levels: Low and High) and Numerical Distance (2 levels: Close and Far). In the Low category, numbers were combined for Close (3 and 4) and Far (1 and 2); the same was done for Close (6 and 7) and Far ( 8 and 9 ) in the High category. For the child data, a within-subjects (repeated-measures) factor Grade (2 levels: kindergarten and grade 1) was included. The ERP (P2p) amplitude analyses included an extra factor Hemisphere (2 levels: Left and Right).

Assumptions were checked using a Compound Symmetry covariance structure, removing single observations as outliers if residuals exceeded three standard deviations. This led to the removal of the following observations (single data points) for children: 1 in the non-symbolic RT data, 4 in the non-symbolic accuracy data, 1 in the symbolic accuracy data, and 2 in the symbolic-ERP data. For adults, the following outliers were removed: 4 in the symbolic RT data, 2 in the symbolic accuracy data, and 3 in the non-symbolic accuracy data. Assumptions of normality and homoscedasticity were met in all analyses. In both children and adults and for dependent variables RT and P2p amplitude, various covariance matrices were compared using AIC to determine the best fitting model. For the children's data, the models for RT showed the best fit when analyzed using an Unstructured Covariance matrix for both symbolic and non-symbolic tasks, whereas for the adult RT data, the compound symmetry matrix showed a better and more parsimonious fit. For the children's P2p-ERP data, the Toeplitz covariance structure showed the best fit, whereas the adult-P2p data fitted best with an Unstructured Covariance matrix. All models were subsequently reduced by removing non-significant four-way and three-way interactions. Non-significant two-way interactions were purposely kept in the model; removing them did not change any of the conclusions. 


\subsection{Results}

Mean reaction time and accuracy results in the four numerical distance $\mathrm{x}$ range conditions in symbolic and non-symbolic tasks from children and adults are presented in Figure 3.2.

\subsubsection{Symbolic Number Comparison Task}

\section{Behavioral results}

Children. A main effect of Grade was present for both reaction time $(F[1,25]=6.91, p$ $=.014)$ and accuracy $\left(\chi_{1}^{2}=8.96, p=.003\right)$; children needed significantly less time and were more accurate to compare the digits with reference number five in grade 1 than one year earlier in kindergarten $\left(\mathrm{RT}: \mathrm{M}_{\text {Diff }}=83.88 \mathrm{~ms} ; t(25)_{\text {Grade1-Kindergarten }}=2.63, p\right.$ $=.014$; Accuracy: $\left.\mathrm{M}_{\text {Diff }}=6.57 \% ; t(1)_{\text {Grade1-Kindergarten }}=3.04, p=.002\right)$. The analyses also yielded main effects of numerical Distance for both reaction time and accuracy (RT: $F[1,25]=54.31, p<.001$; Accuracy: $\left.\chi_{1}^{2}=42.01, p<.001\right)$; number comparison time was faster and more accurate in response to digits with far compared to close distance to the reference number five $\left(\mathrm{RT}: \mathrm{M}_{\text {Diff }}=83.38 \mathrm{~ms} ; t(25)_{\text {Close-Far }}=7.37, p<.001\right.$; Accuracy: $\left.\mathrm{M}_{\text {Diff }}=4.83 \% ; t(1)_{\text {Far-Close }}=6.57, p<.001\right)$. For reaction time nor accuracy main effects or interactions with the factor numerical Range were found (all p's > .209).

Adults. The behavioral analyses in the symbolic task showed main effects of Distance for $\operatorname{RT}(F[1,18]=87.30, p<.001)$ as well as for accuracy $\left(\chi_{1}^{2}=50.77, p<.001\right)$. Both main effects were indicative of typical Numerical Distance Effects (NDE's) with RT being significantly faster and accuracy being significantly higher in far than close distance trials $\left(\mathrm{RT}: \mathrm{M}_{\text {Diff }}=31.28 \mathrm{~ms} ; t(18)_{\text {Close-Far }}=9.34, p<.001 ;\right.$ Accuracy: $\mathrm{M}_{\text {Diff }}=3.83 \% ; t(1)_{\text {Close-Far }}$ $=7.34, p<.001)$. There was no main effect of, or interactions with, the factor numerical Range for RT nor accuracy measures (all p's $>.05$ ). 

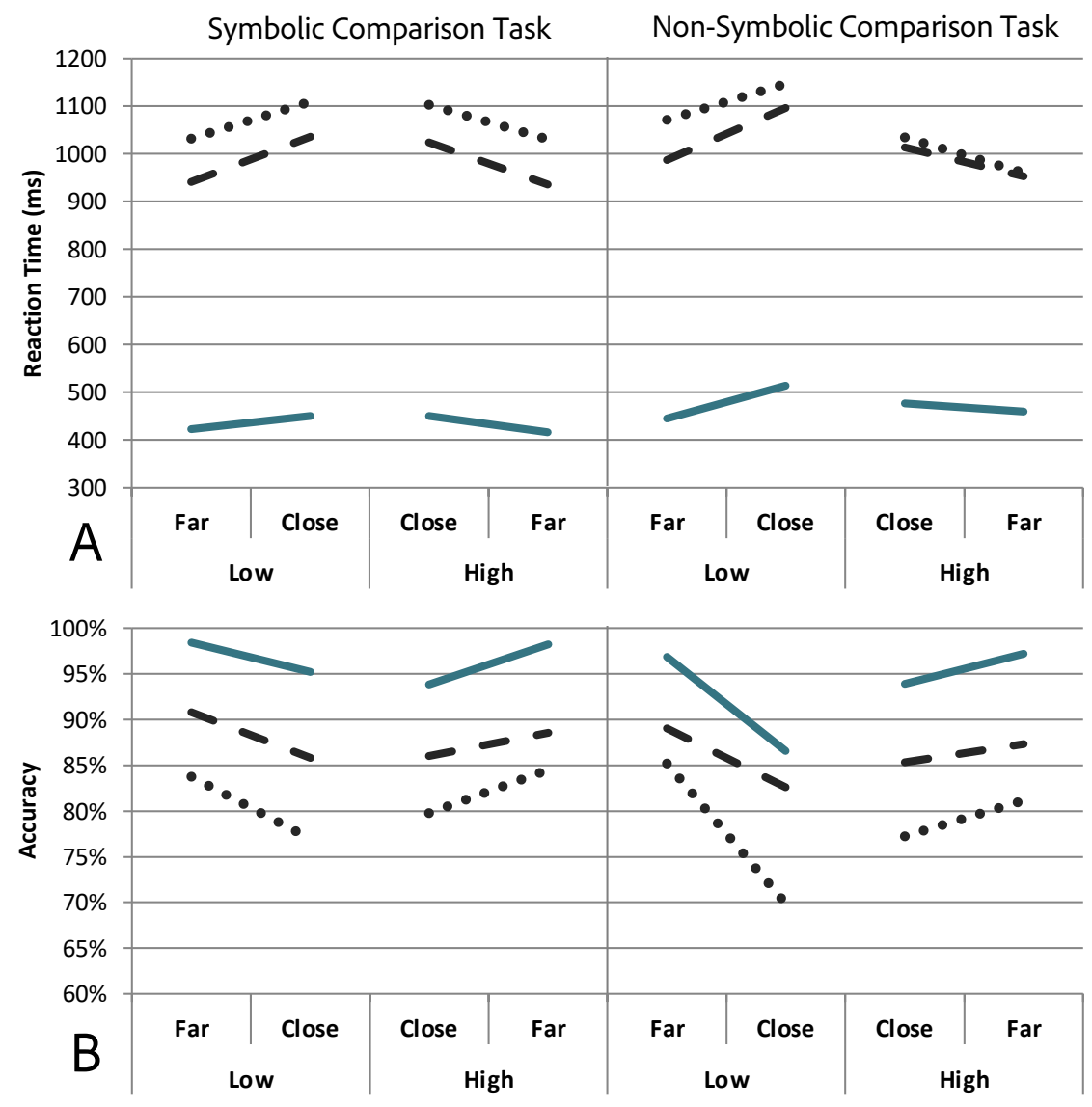

-. Kindergarten (T1) - Grade $1(\mathrm{~T} 2) \quad$ Adults

Figure 3.2: Behavioral data showing mean Reaction Time (A; Top row) and mean Accuracy percentages (B; Bottom row) in the different conditions (far-close numerical distance and low-high numerical ranges) for children (at Kindergarten and Grade 1 age) and adults in Symbolic number comparison task with Arabic digits (left panel) and the Non-Symbolic number comparison task with dot patterns (right panel). 


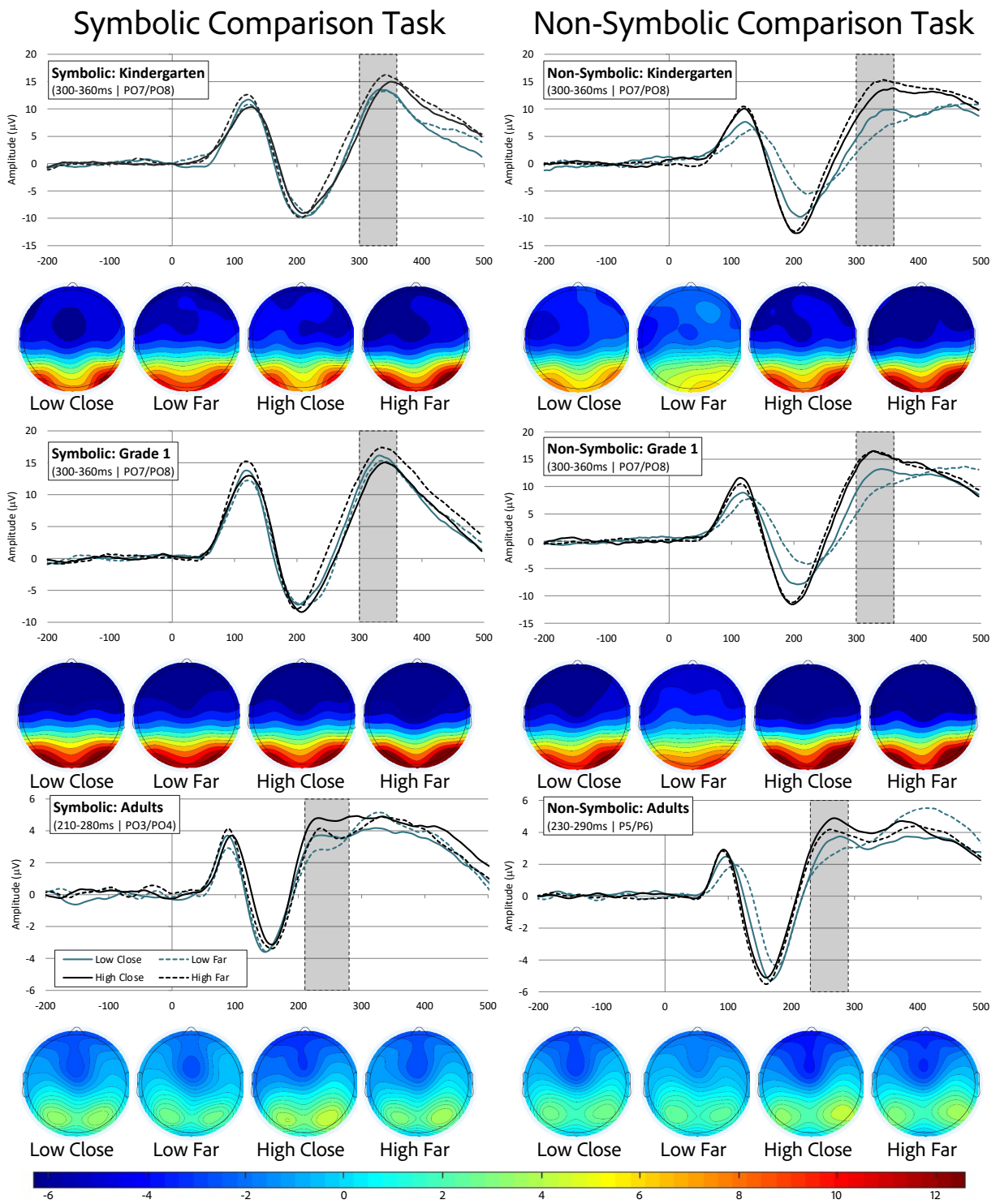

Figure 3.3: Grand Average ERP's (averaged over hemispheres) and Topography plots in Symbolic and NonSymbolic tasks and in the four Range-Distance categories: Low-Close, Low-Far, High-Close, High-Far. The top row shows the ERP data from the children at Kindergarten age (T1), and the middle row shows children's ERP data one year later in Grade 1 (T2); children's P2p is marked by the grey area and was maximal at PO7/PO8 electrodes in a 300-360 ms time-window. The bottom panel shows the grand average ERPs and P2p topo-plots of adults with the $\mathrm{P} 2 \mathrm{p}$ analysis windows marked by the grey bar. In the symbolic task $\mathrm{P} 2 \mathrm{p}$ amplitude was scored in a 210-280 ms window at PO3/PO4 (bottom left), and in the non-symbolic task P2p amplitude was scored in a 230-290 ms time window at P5/P6. (bottom right). 


\section{Symbolic Number Comparison Task: P2p results}

Children. A main effect of Grade $(F[1,70.72]=9.52, p=.003)$ indicated a significant overall increase in children's mean P2p amplitude in the symbolic number comparison task from kindergarten to grade $1\left(\mathrm{M}_{\text {Diff }}=2.57 \mu \mathrm{V} ; t(70.72)_{\text {Grade-Kindergarten }}=3.09, p=\right.$ $.003)$. A main Hemisphere effect $(F[1,320.60]=10.06, p=.002)$, revealed a significant lateralization of $\mathrm{P} 2 \mathrm{p}$ amplitude to the right hemisphere $\left(\mathrm{M}_{\text {Diff }}=1.42 \mu \mathrm{V} ; t(320.60)_{\text {Left-Right }}\right.$ $=3.17, p=.002)$. Besides these main effects, a significant Range*Distance interaction $(F[1,367.75]=18.39, p<.001)$, revealed a small, but typical (close $>$ far $)$, numerical distance effect on P2p amplitude in response to digits in the low (1-4) number range $\left(\mathrm{M}_{\text {Diff }}=1.33 \mu \mathrm{V} ; t(377.45)_{\text {Close-Far }}=1.97, p=.049\right)$, whereas an atypical, reversed (far $>$ small) distance effect on $\mathrm{P} 2 \mathrm{p}$ amplitude was found for higher digits 6-9 $\left(\mathrm{M}_{\text {Diff }}=-2.78 \mu \mathrm{V}\right.$; $t(377.54)_{\text {Close-Far }}=-4.11, p<.001$; see left panel Figure 5). Closer study of the reversed distance effect for the 6-9 number range by looking at the $\mathrm{P} 2 \mathrm{p}$ responses to the four separate digits in the 6-9 range, learned that there was an unexplained dip in P2p amplitude in response to digit 7 (see Figure 4).

It was therefore decided to repeat the analyses excluding digit 7; the high-close category now only including the P2p amplitude in response to number 6 in the nonsubitizing close category. This analysis also revealed a significant main effect for Grade $(F[1,52.01]=8.04, p=.007)$ and a Range*Distance interaction $(F[1,58.32]=4.43, p$ $=.040$ ), but now the distance effect for digits 6 (close) versus 8/9 (far) was no longer significant $\left(\mathrm{M}_{\text {Diff }}=-0.59 \mu \mathrm{V} ; t(116.01)_{\text {Close-Far }}=-0.94, p=.348\right)$. This analysis thus shows that the unexplained dip in children's $\mathrm{P} 2 \mathrm{p}$ response to digit 7 was responsible for the finding of a reversed distance effect in the high range. There were no other significant main or interaction effects (all p-values $>.083$ ).

Summarizing, the results of the symbolic comparison task with Arabic digits 1-9 showed typical numerical distance effects on RT and accuracy (far faster/more accurate than close) in children at both ages and in adults and for both subitizing and non-subitizing ranges. The $\mathrm{P} 2 \mathrm{p}$ amplitude results showed a significant numerical distance effect only for the subitizing (1-4) range in children (no effect of age), whereas adults had a P2p distance effect for numerals in both numerical ranges. 


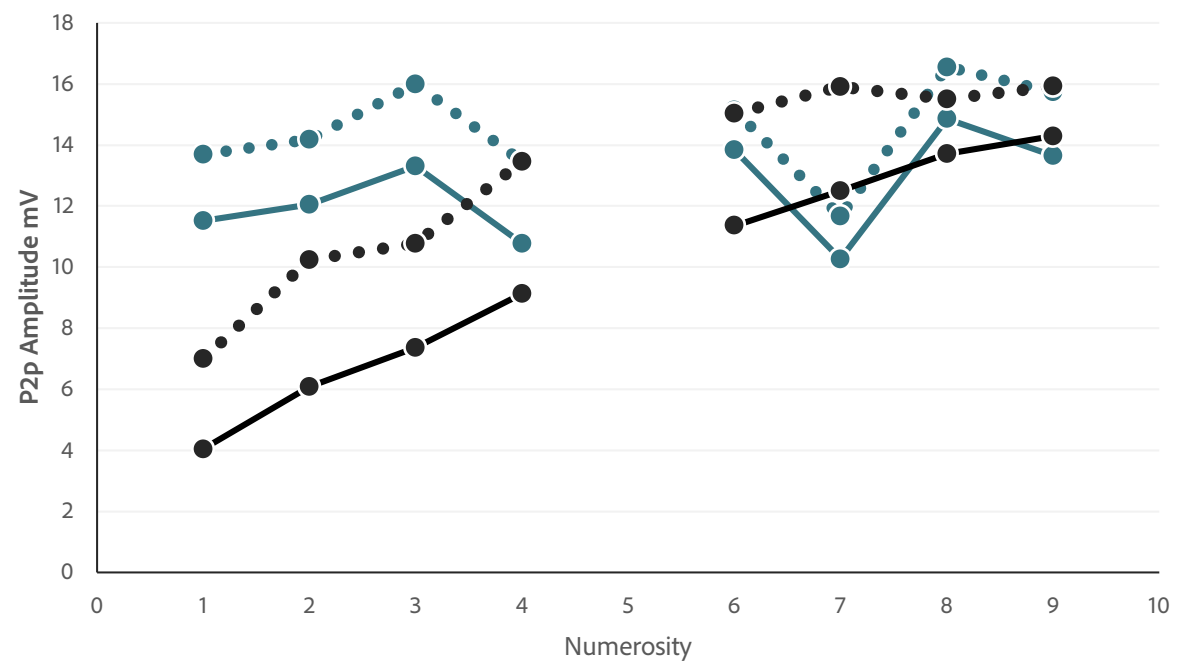

$\longrightarrow$ Symbolic $\longrightarrow$ Non-Symbolic $\longrightarrow$ Kindergarten $(\mathrm{T} 1) \ldots$ Grade 1 (T2)

Figure 3.4: Children's mean $P 2 p$ amplitude averaged over all correctly responded trials in the symbolic number comparison task with Arabic numerals (red) and the non-symbolic comparison task with dot patterns (black) in the subitizing range (1-4) and the non-subitizing range (6-9) at both longitudinal measurement points: kindergarten (solid lines) and Grade 1 (dotted lines). The figure shows the sharp P2p amplitude decrease for Symbol "7" in the symbolic number comparison task.

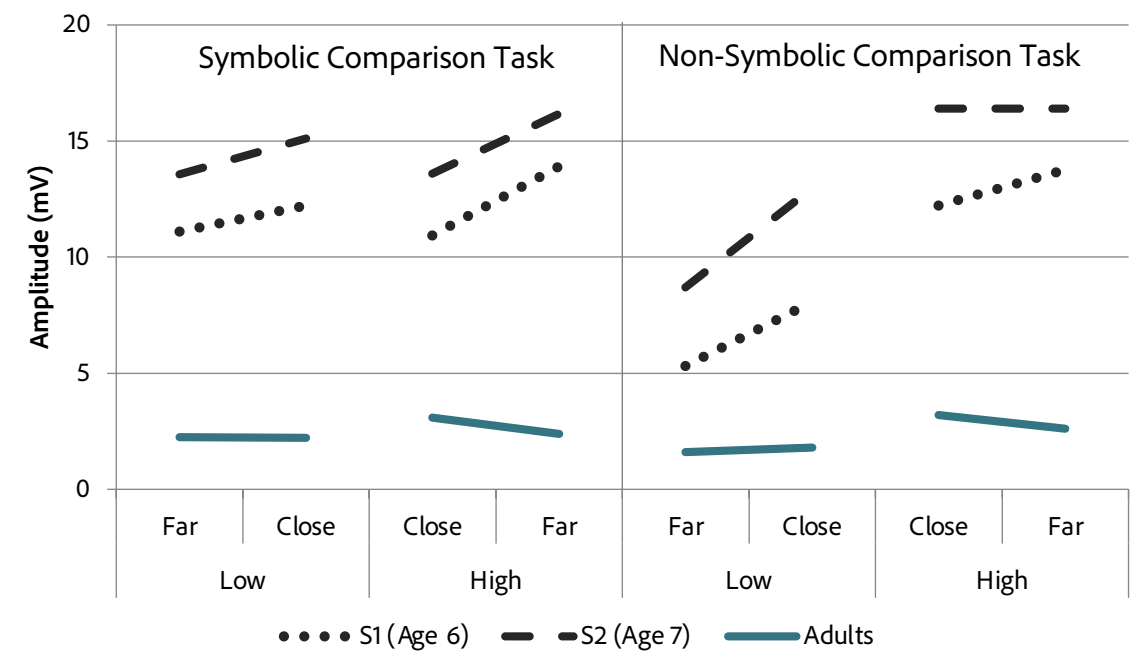

Figure 3.5: Mean P2p amplitude in the different conditions in Symbolic (left) and Non-symbolic (right) number comparison tasks. The P2p values for children at both time points (Kindergarten and Grade 1) depict the mean amplitude computed in a 300-360 ms time window and averaged across PO7/PO8 electrodes. Adult's P2p in the symbolic task depicts the mean amplitude in a 210-280 ms time window averaged across PO3/PO4 electrodes and in the non-symbolic task the P2p was averaged across P5/P6 electrodes in a 230-290 ms time window. 
Adults. The only significant effects on P2p amplitude in the symbolic comparison task were main effects of numerical Range $(F[1,20.54]=10.43, p=.004)$ and number Distance $(F[1,54.07]=33.67, p<.001)$; $\mathrm{P} 2 \mathrm{p}$ amplitude was larger in response to digit stimuli in the non-subitizing 6-9 range than in the subitizing (1-4) range $\left(\mathrm{M}_{\text {Diff }}=0.76 \mu \mathrm{V}\right.$; $\left.t(20.54)_{\text {High-Low }}=3.23, p=.004\right)$ and was larger on close than far distance trials $\left(\mathrm{M}_{\text {Diff }}=\right.$ $\left.0.99 \mu \mathrm{V} ; t(54.07)_{\text {Close-Far }}=5.80, p<.001\right)$, see right panel Figure 5 .

\subsubsection{Non-Symbolic Number Comparison Task}

\section{Behavioral results}

Children. A main effect of Distance was present for reaction time $(F[1,24.05]=61.78, p$ $<.001$ ), showing a typical NDE with faster reaction times in far trials compared to close trials $\left(\mathrm{M}_{\text {Diff }}=79.93 \mathrm{~ms} ; t(24.05)_{\text {Close-Far }}=7.86, p<.001\right)$. Further testing of the significant Grade*Range interaction $(F[1,24.66]=5.13, p=.033)$, indicated that children's reaction times were slower to lower numerosities 1-4 than to higher numerosities 6-9 (irrespective of numerical distance), but this numerical range effect was larger in kindergartners (T1; $\mathrm{M}_{\text {Diff }}$ $\left.=111.79 \mathrm{~ms} ; t(25.16)_{\text {Low-High }}=6.08, p<.001\right)$ than one year later in grade $1\left(\mathrm{~T} 2 ; \mathrm{M}_{\text {Diff }}=\right.$ $\left.58.67 \mathrm{~ms} ; t(25)_{\text {Low-High }}=3.32, p=.003\right)$. None of the other effects reached significance (all p's > .066).

For accuracy a three-way interaction between Grade, Range, and Distance $\left(\chi_{1}^{2}=6.23\right.$, $p=.013)$ was present and further explored by splitting the data for Low (1-4) and High (6-9) numerical ranges. A significant two-way interaction between Grade and Distance $\left(\chi_{1}^{2}=12.84, p<.001\right)$ was present for the Low range; whereas the numerical distance effect (far more accurate than close) was significant at both time points, it was larger in kindergarten $\left(\mathrm{M}_{\text {Diff }}=15.29 \% ; t(1)_{\text {Far-Close }}=6.65, p<.001\right)$ than grade $1\left(\mathrm{M}_{\text {Diff }}=6.44 \%\right.$; $\left.t(1)_{\text {Far-Close }}=4.20, p<.001\right)$. For the higher numerical range only significant main effects of Grade $\left(\chi_{1}^{2}=5.05, p=.025\right)$ and Distance $\left(\chi_{1}^{2}=4.37, p=.037\right)$ were present, indicating an overall increase in accuracy over time $\left(\mathrm{M}_{\text {Diff }}=7.14 \% ; t(1)_{\text {Far-Close }}=2.28, p=.022\right)$ and a typical and equally sized distance effect of higher accuracy for far than close trials at both ages $\left(\mathrm{M}_{\text {Diff }}=3.01 \% ; t(1)_{\text {Far-Close }}=2.09, p=.037\right)$. These results signify: 1$)$ larger numerical distance effects on accuracy when comparing dot patterns in the subitizing than the non-subitizing range and 2) a decrease in the size of the distance effect on accuracy from kindergarten to grade 1 for dot patterns in the subitizing range, whereas the distance effect for higher quantities 6-9 does not show any developmental change between the two studied age ranges. 
Adults. The behavioral analyses in the non-symbolic task showed a two-way interaction between Range and Distance for RT $(F[1,19]=46.86, p<.001)$ as well as for accuracy $\left(\chi_{1}^{2}=10.87, p=.001\right)$. Distance effects were statistically significant in both numerical ranges, but larger in the subitizing range $\left(\mathrm{RT}: \mathrm{M}_{\text {Diff }}=68.37 \mathrm{~ms} ; t(19)_{\text {Close-Far }}=\right.$ $8.37, p<.001$; Accuracy: $\left.\mathrm{M}_{\text {Diff }}=10.26 \% ; t(1)_{\text {Close-Far }}=5.18, p<.001\right)$ compared to the 6-9 number range $\left(\mathrm{RT}: \mathrm{M}_{\text {Diff }}=16.26 \mathrm{~ms} ; t(19)_{\text {Close-Far }}=4.73, p<.001\right.$; Accuracy: $\mathrm{M}_{\text {Diff }}=$ $\left.3.25 \% ; t(1)_{\text {Close-Far }}=4.45, p<.001\right)$.

\section{Non-Symbolic Number Comparison Task: P2p results}

Children. A main effect of Grade $(F[1,53.39]=35.30, p<.001)$ indicated a significant overall increase in children's mean $\mathrm{P} 2 \mathrm{p}$ amplitude in the non-symbolic task from kindergarten to grade $1\left(\mathrm{M}_{\text {Diff }}=3.77 \mu \mathrm{V} ; t(53.39)_{\text {Grade } 1 \text {-Kindergarten }}=5.94, p<.001\right)$. Further testing of the significant Range*Distance interaction $(F[1,51.74]=13.71, p<$ .001 ), revealed a typical (close $>$ far), numerical distance effect on P2p amplitude in response to dots in the low (1-4) number range $\left(\mathrm{M}_{\text {Diff }}=3.52 \mu \mathrm{V} ; t(86.60)_{\text {Close-Far }}=5.15\right.$, $p<.001)$, whereas no distance effect on $\mathrm{P} 2 \mathrm{p}$ amplitude was found for dot stimuli representing numerosities 6-9 $\left(\mathrm{M}_{\text {Diff }}=-0.77 \mu \mathrm{V} ; t(86.60)_{\text {Close-Far }}=-1.13, p=.263\right.$; see right panel Figure 5). There were no other significant main or interaction effects (all $p$-values $>.058)$.

Adults. Significant main effects on the non-symbolic P2p in adults were present for Range $(F[1,19]=28.16, p<.001)$ and Distance $(F[1,19]=12.61, p=.002)$. The Range effect showed that $\mathrm{P} 2 \mathrm{p}$ amplitude was larger in response to dot stimuli representing higher numerosities 6-9 than lower numerosities 1-4 $\left(\mathrm{M}_{\text {Diff }}=1.29 \mu \mathrm{V} ; t(19)_{\text {High-Low }}=5.31, p\right.$ $<.001)$. The Distance effects showed larger P2p amplitude on close than far distance trials $\left(\mathrm{M}_{\text {Diff }}=0.49 \mu \mathrm{V} ; t(19)_{\text {Close-Far }}=3.55, p=.002\right)$, see right panel Figure 5. The Range*Hemisphere interaction was significant as well $(F[1,19]=4.86, p=.040)$; pairwise comparisons indicated no significant hemisphere differences in either range (Low $p=$ .559 ; High $p=.193)$, but a slightly larger range effect in the right hemisphere $\left(\mathrm{M}_{\text {Diff }}=\right.$ $\left.1.63 \mu \mathrm{V} ; t(19)_{\text {High-Low }}=4.99, p<.001\right)$ compared to the left hemisphere $\left(\mathrm{M}_{\text {Diff }}=0.96 \mu \mathrm{V}\right.$; $\left.t(19)_{\text {High-Low }}=3.98, p=.001\right)$. 
Table 3.1: Means and Standard Errors (between brackets) for behavioral data in the different Stimulus-Range conditions separate for Grade and Distance

\begin{tabular}{|c|c|c|c|c|}
\hline & & & Distance Close & Distance Far \\
\hline \multirow{12}{*}{$\begin{array}{l}\text { Reaction Time } \\
\text { (millisecond; ms) }\end{array}$} & \multirow{4}{*}{ Kindergarten } & Symbolic Low & $1109.63(47.46)$ & $1031.66(42.88)$ \\
\hline & & Symbolic High & $1102.75(46.53)$ & $1030.59(44.16)$ \\
\hline & & Non-Symbolic Low & $1147.14(48.26)$ & $1071.26(46.40)$ \\
\hline & & Non-Symbolic High & $1034.27(44.16)$ & $960.54(37.64)$ \\
\hline & \multirow{4}{*}{ First Grade } & Symbolic Low & $1036.46(52.85)$ & $941.86(42.33)$ \\
\hline & & Symbolic High & $1024.79(49.20)$ & $936.01(44.99)$ \\
\hline & & Non-Symbolic Low & $1096.41(47.15)$ & $986.98(43.55)$ \\
\hline & & Non-Symbolic High & $1013.36(44.61)$ & $952.69(40.05)$ \\
\hline & \multirow{4}{*}{ Adults } & Symbolic Low & $450.46(8.90)$ & $422.42(7.13)$ \\
\hline & & Symbolic High & $450.90(8.37)$ & $416.38(6.89)$ \\
\hline & & Non-Symbolic Low & $514.02(15.43)$ & $445.65(10.62)$ \\
\hline & & Non-Symbolic High & $476.59(13.12)$ & $460.33(12.49)$ \\
\hline \multirow{12}{*}{$\begin{array}{l}\text { Accuracy } \\
(\% \text { Correct })\end{array}$} & \multirow{4}{*}{ Kindergarten } & Symbolic Low & $76.94 \%(2.63 \%)$ & $83.78 \%(2.16 \%)$ \\
\hline & & Symbolic High & $79.77 \%(2.49 \%)$ & $84.50 \%(2.41 \%)$ \\
\hline & & Non-Symbolic Low & $69.90 \%(2.97 \%)$ & $85.19 \%(2.24 \%)$ \\
\hline & & Non-Symbolic High & $77.21 \%(2.72 \%)$ & $81.15 \%(2.88 \%)$ \\
\hline & \multirow{4}{*}{ First Grade } & Symbolic Low & $85.78 \%(1.49 \%)$ & $90.80 \%(1.67 \%)$ \\
\hline & & Symbolic High & $85.99 \%(2.14 \%)$ & $88.55 \%(1.72 \%)$ \\
\hline & & Non-Symbolic Low & $82.60 \%(2.07 \%)$ & $89.04 \%(1.47 \%)$ \\
\hline & & Non-Symbolic High & $85.31 \%(2.33 \%)$ & $87.30 \%(2.10 \%)$ \\
\hline & \multirow{4}{*}{ Adults } & Symbolic Low & $95.19 \%(0.77 \%)$ & $98.42 \%(0.40 \%)$ \\
\hline & & Symbolic High & $93.83 \%(0.88 \%)$ & $98.25 \%(0.45 \%)$ \\
\hline & & Non-Symbolic Low & $86.57 \%(2.08 \%)$ & $96.83 \%(0.75 \%)$ \\
\hline & & Non-Symbolic High & $93.92 \%(0.97 \%)$ & $97.17 \%(0.60 \%)$ \\
\hline
\end{tabular}

Values shown are estimated marginal means with Standard Errors.

Low: Close $=$ average of $3 \& 4$ and Far $=$ average of $1 \& 2$; High: Close $=$ average of $6 \& 7$ and Far $=$ average of $8 \& 9$

Table 3.2: Means and Standard Errors (between brackets) for ERP data in the four different Stimulus-Range conditions separate for Grade, Distance, and hemisphere.

\begin{tabular}{|c|c|c|c|c|c|c|}
\hline & & & \multicolumn{2}{|c|}{ Distance Close } & \multicolumn{2}{|c|}{ Distance Far } \\
\hline & & & Left & Right & Left & Right \\
\hline \multirow{8}{*}{$\begin{array}{l}\text { P2p amplitude }(\mu \mathrm{V}) \\
\text { PO7 \& PO8 300-360 ms }\end{array}$} & \multirow{4}{*}{ Kindergarten } & Symbolic Low & $11,28(1,77)$ & $13,07(1,78)$ & $10,51(1,73)$ & $11,73(1,72)$ \\
\hline & & Symbolic High & $11,97(1,77)$ & $14,27(1,76)$ & $13,05(1,76)$ & $14,79(1,79)$ \\
\hline & & Non-Symbolic Low & $7.94(1.76)$ & $8.21(1.72)$ & $4.55(1.68)$ & $6.08(1.69)$ \\
\hline & & Non-Symbolic High & $11.64(1.71)$ & $12.82(1.69)$ & $12.53(1.70)$ & $14.98(1.74)$ \\
\hline & \multirow{4}{*}{ First Grade } & Symbolic Low & $14.25(1.79)$ & $15.90(1.76)$ & $13.06(1.75)$ & $14.16(1.76)$ \\
\hline & & Symbolic High & $14.65(1.73)$ & $16.82(1.73)$ & $15.32(1.78)$ & $16.93(1.77)$ \\
\hline & & Non-Symbolic Low & $11.66(1.74)$ & $14.29(1.70)$ & $7.83(1.69)$ & $9.56(1.71)$ \\
\hline & & Non-Symbolic High & $14.61(1.69)$ & $18.15(1.68)$ & $15.07(1.72)$ & $17.72(1.76)$ \\
\hline P2p amplitude $(\mu \mathrm{V})$ & \multirow{4}{*}{ Adults } & Symbolic Low & $3.50(1.11)$ & $3.69(1.11)$ & $2.54(1.11)$ & $2.72(1.11)$ \\
\hline $\begin{array}{l}\text { PO3 \& PO4: 210-280 ms } \\
\text { Symbolic }\end{array}$ & & Symbolic High & $4.09(1.11)$ & $4.66(1.11)$ & $3.09(1.11)$ & $3.65(1.11)$ \\
\hline P5 \& P6: $230-290 \mathrm{~ms}$ & & Non-Symbolic Low & $2.54(0.60)$ & $3.09(0.84)$ & $2.10(0.67)$ & $2.33(0.83)$ \\
\hline Non-Symbolic & & Non-Symbolic High & $3.38(0.52)$ & $4.61(0.97)$ & $3.17(0.67)$ & $4.07(0.98)$ \\
\hline
\end{tabular}

Values shown are estimated marginal means with Standard Errors.

Low: Close $=$ average of $3 \& 4$ and $\mathrm{Far}=$ average of $1 \& 2$; High: $\mathrm{Close}=$ average of $6 \& 7$ and $\mathrm{Far}=$ average of $8 \& 9$ 


\subsection{Discussion}

The main aim question of the present study was to investigate if distance effects on performance and especially the amplitude of the P2p-ERP component in symbolic and non-symbolic comparison tasks would overlap or diverge across development, and whether there is a difference herein between subitizing and non-subitizing ranges. Based on recent theories and findings in the developmental and adult number processing literature (see introduction), we hypothesized that 5-6 year old children would only show symbolic - non-symbolic mapping for Arabic digits in the subitizing range, whereas other processes/mechanisms, such as linguistically based semantics (Krajcsi and Kojouharova, 2017; Krajcsi et al., 2016a) or a-semantic processes (Cohen, 2009; Cohen et al., 2013; Lin and Göbel, 2019; Reynvoet and Sasanguie, 2016) were assumed to underlie the learning of higher number symbols 6-9 outside the subitizing range. The P2p component has in previous number processing studies been shown to be sensitive to numerical manipulations such as numerical distance and has been localized mainly to right intraparietal regions (Dehaene, 1996; Hyde and Spelke, 2012), a region that has in fMRI studies been linked to representing and comparing quantities (Dehaene, 2009; Dehaene et al., 2003). Effects of numerical distance on the P2p component have therefore been suggested to index early numeric semantic processing associated with the automatic activation of non-symbolic magnitude information linked to the to-be-processed number stimulus (Dehaene, 1996; Hyde and Spelke, 2012; Libertus et al., 2007; Temple and Posner, 1998). Hence, overlap in P2p distance effects between symbolic and non-symbolic tasks across development were in the current study interpreted as a sign of shared and automatically activated analog magnitude representations.

In the symbolic number comparison task adults and children (at both ages) showed a numerical distance effect (NDE) for Arabic digits in- and outside the subitizing range on reaction time and accuracy (main effects). However, children only showed an effect of numerical distance on P2p amplitude for the 1-4 subitizing range, whereas adults showed a $\mathrm{P} 2 \mathrm{p}$ distance effect for both numerical ranges. When adopting the view of P2p distance effects being a sign of access/activation of analog quantity representations with which the symbol is associated (Dehaene, 1996; Hyde and Spelke, 2009, 2012; Libertus et al., 2007; Pinel et al., 2001), this would mean that children did activate analog magnitude representations during comparison of Arabic digits 1-4 to referent number five $(\mathrm{P} 2 \mathrm{p}$ distance effect present), but not for digits 6-9 (no P2p distance effect). In the same way, the presence of a P2p-NDE for digits beyond the subitizing range in adults would then mean that adults did activate analog magnitude representations during comparison of digits in 
and beyond the subitizing range. Whereas subitizing has been reported to only occur for the first three or four quantities, a study by Sullivan \& Barner (2013) has provided behavioral evidence for associative, exact, number word - quantity mapping for numbers up to twelve in adults, whereas this was only up to six in 5-7-year-old children (Sullivan and Barner, 2014). Although these studies included number words, it is assumable that adults will have acquired the same extended associative symbol - non-symbolic quantity mappings for the first 10 Arabic digits, explaining the presence of a symbolic P2p distance effect in adults for both 1-4 and 6-9 ranges and only for digits 1-4 in children. Whereas 5-6-year-old children might know the cardinality of number words above four and can cite the counting list up till number 10, this does not imply full understanding of the magnitude associated with Arabic digits (Davidson et al., 2012; Le Corre, 2014; Sella et al., 2017). In several studies, Sella et al. $(2017 ; 2018,2019)$ have shown that 4-6-year-old children who can order digits in a correct and linear order have an advanced understanding of the magnitude associated with the numbers as measured by their number comparison performance. The current P2p data might thus argue for less mature ordinal/spatial representations of Arabic digits 6-9 on the number line in our 5-6-year-old children, complicating associative mapping between Arabic digits 6-9 and their corresponding magnitudes, possibly explaining the absent P2p distance effects for digits 6-9 in children.

An important question is why children did show distance effects for Arabic digits in and beyond the subitizing range on performance measures (reaction time, accuracy), whereas this was not the case for P2p amplitude. If not via analog magnitude representations, how can we then explain the distance effect on reaction time and accuracy for higher Arabic digits 6-9 in children? As was mentioned in the introduction, for many years, the dominant view has been that distance effects on reaction time or accuracy in number symbol comparison tasks are a sign of digits being represented in the ANS, the distance effect being rooted in the numerical distance between of the numbers. However, multiple authors have suggested other possible processes/mechanisms underlying distance effects in symbol (number words/Arabic digits) comparison or mapping tasks. For example, distance effects on reaction time in number symbol comparison/matching tasks have also been suggested to occur via an a-semantic (non-numeric) route, such as on the basis of physical similarities/differences, as suggested by results from studies in adults with symbol-symbol (word-digit) mapping tasks (Cohen, 2009; Cohen et al., 2013; Lin and Göbel, 2019). Others have suggested that numerals (Arabic digits) are represented in a semantic association network, the distance effect originating from the strength of associations between the digits and larger semantic property categories like 'small' and 
'large' (Krajcsi and Kojouharova, 2017; Krajcsi et al., 2016b). For example, digit '1' will have developed a stronger association with the category "small" than digit '4' and one will hence be more accurate/faster to decide that 1 is smaller than five, causing a distance effect. Since in the currently used symbol-to-referent task one only had to make smaller/larger than five comparisons/decisions, distance effects could be caused by the digits' associative strength with categories smaller and larger instead of the numeric distance between the digits themselves. The reliance on such semantic (linguistic) associative links in the case of symbol-symbol comparisons might be larger for digits outside the subitizing range because in this range exact quantity mapping is complicated because of noisy (not exact) representations. Multiple studies in preschoolers support the difficulty of symbol - quantity mapping in the non-subitizing range by reporting earlier and more accurate symbol-quantity mapping for small (1-3) numbers in the subitizing range than for medium/larger numbers (4-9) for which it is more difficult to determine cardinality (Benoit et al., 2013; Hurst et al., 2016; Lira et al., 2017; Odic et al., 2015). Also, in older children and adults there is evidence for one-to-one number word - quantity mapping in the subitizing range, but not for number words in the non-subitizable range (Šetić Beg et al., 2021; Sullivan and Barner, 2013, 2014). Even when number words and quantity (number of objects) were only implicitly processed, adults showed facilitated processing in a sentence-picture matching task when number words and quantity were congruent with each other, but only in the subitizing range, pointing to shared and automatically activated number-word - quantity representations in the subitizable range (Šetić Beg et al., 2021). Finally, in a previous ERP study in which we mimicked number symbol learning in adults by letting them perform a novel symbol - quantity mapping task with feedback (van den Berg et al., 2020), we found that the amplitude of the P2p was only modulated by learning of novel symbols representing quantities 1-4 that one could successfully map onto their corresponding quantities by subitizing, and not for quantities 6-9 that could, due to the short presentation time, not be exactly enumerated. 
One main question of the present study was if the distance effects on the P2p-ERP component (and performance) in the symbolic and non-symbolic tasks would show different developmental paths, and whether there is a difference herein between subitizing and non-subitizing ranges. Children showed similar distance effects on P2p amplitude in both tasks, with these effects being only present for the subitizing range and not showing any developmental change between kindergarten to a year later in grade 1. Adults also showed similar distance effects on P2p amplitude that were in both tasks present for both numerical ranges. Distance effects on performance measures (reaction time or accuracy) in symbolic and non-symbolic tasks did however show some developmental differences. That is, whereas in both tasks children and adults showed distance effects on reaction time and accuracy for both numerical ranges (as discussed above), children showed a distance* range*age interaction for accuracy in the non-symbolic task, whereas no age effects were found in the symbolic task. Further testing of the interaction showed that non-symbolic distance effects were larger in the subitizing than the non-subitizing range and the size of the NDE for quantities 1-4 decreased from kindergarten to grade 1. A distance*range interaction for reaction time in adults also showed larger NDE's in the subitizing range. These results in children and adults point to easier magnitude comparisons between higher quantities 6-9 than for quantities 1-4 in the subitizing range. This is in line with studies reporting higher attention being involved in subitizing (served by the OTS; Feigenson et al., 2004) than estimation processes (Anobile, Cicchini, et al., 2012; Anobile, Turi, et al., 2012; Burr et al., 2010). The latter used for enumeration of higher quantities when counting is not possible, as is the case with the short stimulus presentation times used in the current task. Concluding, whereas the P2p-distance results are congruent with the view of number symbols 1-4 sharing magnitude representations with quantities 1-4, performance data show developmental effects in the subitizing range that are only present in the non-symbolic task. It is however important to note that to have enough trials for ERP analysis in each condition, in the current comparison-to-referent task (with number five as internal referent number), close and far distances were computed by averaging across the two smallest and largest numbers within both numerical ranges. So, in the subitizing range 'far' was computed by averaging accuracy/reaction time across numerals/quantities 1 and 2 and 'close' across numerals/quantities 3 and 4 . It might consequently be the case that the larger distance effects for dot arrays 1-4 are primarily caused by relatively larger difficulties discriminating dot array four from the referent because it may be just exceeding the subitizing range, marking the transition between subitizing and estimation processes. In Figure 4, the P2p data are depicted for all eight 
numbers separately and P2p amplitudes to digits/quantities 1-3 do show very similar patterns, whereas the $\mathrm{P} 2 \mathrm{p}$ amplitude pattern clearly starts to diverge between tasks for number 4. We also found evidence for more difficult non-symbolic mapping of symbol 4 in a previous ERP study (van den Berg et al., 2020). In this study we mimicked symbol learning in adults by presenting them with a trial-and-error learning task in which the numeric meaning of novel abstract symbols had to be acquired by associating them to their corresponding quantities (shown as dot arrays) on the basis feedback. Also in this study, novel symbol 4 showed more difficult mapping/learning than symbols 1-3; after three learning blocks mapping reaction time and accuracy was similar for symbols 1-3 but still worse for novel symbol 4. Finally, studies in 3-4-year-old preschoolers also provide evidence for faster and easier non-symbolic - symbolic mapping for number symbols (words/digits) 1-3 (Benoit et al., 2013; Hurst et al., 2016; Lira et al., 2017; Odic et al., 2015).

Concluding, the current $\mathrm{P} 2 \mathrm{p}$ and behavioral data from children and adults is supportive of symbolic - non-symbolic mapping only taking place for Arabic digits/quantities in the subitizing range, at least for digits 1-3, supporting the exact mapping hypothesis (Carey, 2009a; Carey and Barner, 2019). The P2p distance data for digits/quantities 6-9 in the non-subitizing range show clearly divergent developmental patterns, with distance effects on $\mathrm{P} 2 \mathrm{p}$ amplitude in this range (pointing to symbolic-non-symbolic mapping) only being present in adults and not in 5-6-year-old children who are just starting to learn numbers. These data suggest that number symbols beyond the subitizing range are learned via other processes than non-symbolic mapping.

\footnotetext{
Acknowledgements

The authors thank Linda Essers for the programming of the tasks and the coordination of/contribution to the collection of the children's data. We further thank all children, parents and schools for their participation. This study was funded by the NWO (National Dutch Organization for Scientific Research) under grant number PROO 411-07-112.
} 


\subsection{References}

Anobile, G., Cicchini, G. M., \& Burr, D. C. (2012). Linear mapping of numbers onto space requires attention. Cognition, 122(3), 454-459. https://doi.org/10.1016/j. cognition.2011.11.006 (cited on page 121)

Anobile, G., Turi, M., Cicchini, G. M., \& Burr, D. C. (2012). The effects of cross-sensory attentional demand on subitizing and on mapping number onto space. Vision Research, 74, 102-109. https://doi.org/10.1016/j.visres.2012.06.005 (cited on page 121)

Barth, H., La Mont, K., Lipton, J., \& Spelke, E. S. (2005). Abstract number and arithmetic in preschool children. Proceedings of the National Academy of Sciences of the United States of America, 102(39), 14116-14121. https://doi.org/10.1073/pnas. 0505512102 (cited on page 99)

Benoit, L., Lehalle, H., Molina, M., Tijus, C., \& Jouen, F. (2013). Young children's mapping between arrays, number words, and digits tt -. Cognition TA, 129(1), 95-101 (cited on pages 101, 120, 122).

Buckley, P. B., \& Gillman, C. B. (1974). Comparisons of digits and dot patterns. Journal of experimental psychology, 103(6), 1131-1136. https://doi.org/10.1037/h0037361 (cited on page 98)

Burr, D. C., Turi, M., \& Anobile, G. (2010). Subitizing but not estimation of numerosity requires attentional resources. Journal of Vision, 10(6), 20-20. https://doi.org/10. 1167/10.6.20 (cited on page 121)

Carey, S. (2009a). The origin of concepts (Volume 133). Oxford University Press. https: //doi.org/10.1093/acprof:oso/9780195367638.001.0001. (Cited on pages 98, 122)

Carey, S. (2009b). The Origin of Concepts (Volume 133). Oxford University Press. https: //doi.org/10.1093/acprof:oso/9780195367638.001.0001. (Cited on pages 101, 104)

Carey, S., \& Barner, D. (2019). Ontogenetic origins of human integer representations. Trends in Cognitive Sciences, 23(10), 823-835. https://doi.org/10.1016/j.tics. 2019.07.004 (cited on pages 98, 100, 101, 104, 122)

Cohen, D. J. (2009). Integers do not automatically activate their quantity representation. Psychonomic Bulletin and Review, 16(2), 332-336. https://doi.org/10.3758/PBR. 16.2.332 (cited on pages 102, 118, 119)

Cohen, D. J., Warren, E., \& Blanc-Goldhammer, D. (2013). Cross-format physical similarity effects and their implications for the numerical cognition architecture. 
Cognitive Psychology, 66(4), 355-379. https://doi.org/10.1016/j.cogpsych.2013.03.001 (cited on pages 102, 118, 119)

Davidson, K., Eng, K., \& Barner, D. (2012). Does learning to count involve a semantic induction? Cognition, 123(1), 162-173. https://doi.org/10.1016/j.cognition.2011. 12.013 (cited on page 119)

Dehaene, S. (1992). Varieties of numerical abilities. Cognition, 44(1-2), 1-42. https : //doi.org/10.1016/0010-0277(92)90049-N (cited on page 99)

Dehaene, S. (1996). The organization of brain activations in number comparison: Event-related potentials and the additive-factors method. Journal of Cognitive Neuroscience, 8(1), 47-68. https://doi.org/10.1162/jocn.1996.8.1.47 (cited on pages $102,108,118)$

Dehaene, S. (2001, February). Précis of the number sense. Mind \& language (Pages 16-36). https://doi.org/10.1111/1468-0017.00154. (Cited on pages 98, 99)

Dehaene, S. (2009). Origins of mathematical intuitions: The case of arithmetic. Annals of the New York Academy of Sciences, 1156, 232-259. https://doi.org/10.1111/j. 1749-6632.2009.04469.x (cited on page 118)

Dehaene, S. (2011). The number sense. how the mind creates mathematics, revised and updated edition. Oxford University Press. https://doi.org/10.2307/2589308. (Cited on page 99)

Dehaene, S., Dupoux, E., \& Mehler, J. (1990). Is numerical comparison digital? analogical and symbolic effects in two-digit number comparison. Journal of Experimental Psychology: Human Perception and Performance, 16(3), 626-641. https://doi. org/10.1037/0096-1523.16.3.626 (cited on page 99)

Dehaene, S., Izard, V., \& Piazza, M. (2005). Control over non-numerical parameters in numerosity experiments (cited on page 106).

Dehaene, S., Piazza, M., Pinel, P., \& Cohen, L. (2003). Three parietal circuits for number processing. Cognitive Neuropsychology, 20(3-6), 487-506. https://doi.org/10. 1080/02643290244000239 (cited on page 118)

Dehaene, S., Tzourio, N., Frak, V., Raynaud, L., Cohen, L., Mehler, J., \& Mazoyer, B. (1996). Cerebral activations during number multiplication and comparison: A pet study. Neuropsychologia, 34(11), 1097-1106. https://doi.org/10.1016/00283932(96)00027-9 (cited on page 106)

Di Giorgio, E., Lunghi, M., Rugani, R., Regolin, L., Dalla Barba, B., Vallortigara, G., \& Simion, F. (2019). A mental number line in human newborns. Developmental Science, 22(6). https://doi.org/10.1111/desc.12801 (cited on page 98) 
Duncan, E. M., \& McFarland, C. E. (1980). Isolating the effects of symbolic distance and semantic congruity in comparative judgments: An additive-factors analysis. Memory \& cognition, 8(6), 612-622. https://doi.org/10.3758/BF03213781 (cited on page 99)

Feigenson, L., Carey, S., \& Hauser, M. (2002). The representations underlying infants' choice of more: Object files versus analog magnitudes. Psychological Science, 13(2), 150-156. https://doi.org/10.1111/1467-9280.00427 (cited on page 101)

Feigenson, L., Dehaene, S., \& Spelke, E. (2004). Core systems of number. Trends in Cognitive Sciences, 8(7), 307-314. https://doi.org/10.1016/j.tics.2004.05.002 (cited on pages 98, 101, 121)

Geary, D. C., Berch, D. B., \& Koepke, K. M. (2015). Mathematical cognition and learning: Evolutionary origins and early development of number processing. (Cited on page 98).

Gómez-Velázquez, F. R., Berumen, G., \& González-Garrido, A. A. (2015). Comparisons of numerical magnitudes in children with different levels of mathematical achievement. an erp study. Brain Research, 1627, 189-200. https://doi.org/10.1016/j.brainres.2015.09.009 (cited on page 103)

Gouet, C., Gutiérrez Silva, C. A., Guedes, B., \& Peña, M. (2018). Cognitive and neural effects of a brief nonsymbolic approximate arithmetic training in healthy first grade children. Frontiers in integrative neuroscience, 12, 28. https://doi.org/10. 3389/fnint.2018.00028 (cited on page 103)

Habermann, S., Donlan, C., Göbel, S. M., \& Hulme, C. (2020). The critical role of arabic numeral knowledge as a longitudinal predictor of arithmetic development. Journal of Experimental Child Psychology, 193, 104794. https://doi.org/10.1016/j.jecp. 2019.104794 (cited on page 100)

Halberda, J., \& Feigenson, L. (2008). Developmental change in the acuity of the "number sense": The approximate number system in 3-, 4-, 5-, and 6-year-olds and adults. Developmental Psychology, 44(5), 1457-1465. https://doi.org/10.1037/a0012682 (cited on page 99)

Heine, A., Tamm, S., Wißmann, J., \& Jacobs, A. M. (2011). Electrophysiological correlates of non-symbolic numerical magnitude processing in children: Joining the dots. Neuropsychologia, 49(12), 3238-3246. https://doi.org/10.1016/j.neuropsychologia.2011.07.028 (cited on page 103)

Heine, A., Wißmann, J., Tamm, S., De Smedt, B., Schneider, M., Stern, E., Verschaffel, L., \& Jacobs, A. M. (2013). An electrophysiological investigation of non-symbolic 
magnitude processing: Numerical distance effects in children with and without mathematical learning disabilities. Cortex, 49(8), 2162-2177. https://doi.org/10. 1016/j.cortex.2012.11.009 (cited on page 103)

Holloway, I. D., \& Ansari, D. (2009). Mapping numerical magnitudes onto symbols: The numerical distance effect and individual differences in children's mathematics achievement. Journal of Experimental Child Psychology, 103(1), 17-29. https: //doi.org/10.1016/j.jecp.2008.04.001 (cited on page 99)

Hurst, M., Anderson, U., \& Cordes, S. (2016). Mapping among number words, numerals, and nonsymbolic quantities in preschoolers. Journal of Cognition and Development, 18(1), 41-62. https://doi.org/10.1080/15248372.2016.1228653 (cited on pages 101, 120, 122)

Hutchison, J. E., Ansari, D., Zheng, S., De Jesus, S., \& Lyons, I. M. (2019). The relation between subitizable symbolic and non-symbolic number processing over the kindergarten school year. Developmental Science, 00:e12884. https://doi.org/10. 1111/desc.12884 (cited on page 101)

Hyde, D. C., \& Spelke, E. S. (2009). All numbers are not equal: An electrophysiological investigation of small and large number representations. Journal of cognitive neuroscience, 21(6), 1039-1053. https://doi.org/10.1162/jocn.2009.21090 (cited on pages 102,118$)$

Hyde, D. C., \& Spelke, E. S. (2012). Spatiotemporal dynamics of processing nonsymbolic number: An event-related potential source localization study. Human Brain Mapping, 33(9), 2189-2203. https://doi.org/10.1002/hbm.21352 (cited on pages 102,118$)$

Kaufman, E. L., Lord, M. W., Reese, T., \& Volkmann, J. (1949). The discrimination of visual number. The American journal of psychology, 62(4), 498-525. https: //doi.org/10.2307/1418556 (cited on page 101)

Knudsen, B., Fischer, M. H., Henning, A., \& Aschersleben, G. (2015). The development of arabic digit knowledge in 4- to 7-year-old children. Journal of Numerical Cognition, 1(1), 21-37. https://doi.org/10.5964/jnc.v1i1.4 (cited on page 104)

Krajcsi, A., \& Kojouharova, P. (2017). Symbolic numerical distance effect does not reflect the difference between numbers. Frontiers in Psychology, 8(NOV), 2013. https://doi.org/10.3389/fpsyg.2017.02013 (cited on pages 102, 118, 120)

Krajcsi, A., Lengyel, G., \& Kojouharova, P. (2016a). The source of the symbolic numerical distance and size effects. Frontiers in Psychology, 7, 1795. https://doi.org/10. 3389/fpsyg.2016.01795 (cited on pages 102, 118) 
Krajcsi, A., Lengyel, G., \& Kojouharova, P. (2016b). The source of the symbolic numerical distance and size effects. Frontiers in Psychology, 7, 1795. https://doi.org/10. 3389/fpsyg.2016.01795 (cited on page 120)

Le Corre, M. (2014). Children acquire the later-greater principle after the cardinal principle. British Journal of Developmental Psychology, 32(2), 163-177. https://doi.org/10. 1111/bjdp.12029 (cited on page 119)

Leibovich, T., \& Ansari, D. (2016). The symbol-grounding problem in numerical cognition: A review of theory, evidence, and outstanding questions. Canadian Journal of Experimental Psychology, 70(1), 12-23. https://doi.org/10.1037/cep0000070 (cited on pages 98, 100)

Leibovich, T., Katzin, N., Harel, M., \& Henik, A. (2017). From "sense of number" to "sense of magnitude": The role of continuous magnitudes in numerical cognition. Behavioral and Brain Sciences, 40. https://doi.org/10.1017/S0140525X16000960 (cited on page 98)

Libertus, M. E., Woldorff, M. G., \& Brannon, E. M. (2007). Electrophysiological evidence for notation independence in numerical processing. (Volume 3). https://doi.org/ 10.1186/1744-9081-3-1. (Cited on pages 102, 106, 108, 118)

Lin, C. Y., \& Göbel, S. M. (2019). Arabic digits and spoken number words: Timing modulates the cross-modal numerical distance effect. Quarterly Journal of Experimental Psychology, 72(11), 2632-2646. https://doi.org/10.1177/1747021819854444 (cited on pages 102, 118, 119)

Lira, C. J., Carver, M., Douglas, H., \& LeFevre, J. A. (2017). The integration of symbolic and non-symbolic representations of exact quantity in preschool children. Cognition, 166, 382-397. https://doi.org/10.1016/j.cognition.2017.05.033 (cited on pages $101,120,122)$

Lyons, I. M., Ansari, D., \& Beilock, S. L. (2012). Symbolic estrangement: Evidence against a strong association between numerical symbols and the quantities they represent. Journal of experimental psychology. General, 141(4), 635-41. https: //doi.org/10.1037/a0027248 (cited on page 100)

Lyons, I. M., Bugden, S., Zheng, S., De Jesus, S., \& Ansari, D. (2018). Symbolic number skills predict growth in nonsymbolic number skills in kindergarteners. Developmental Psychology, 54(3), 440-457. https://doi.org/10.1037/dev0000445 (cited on page 99) 
Maloney, E. A., Risko, E. F., Ansari, D., \& Fugelsang, J. (2010). Mathematics anxiety affects counting but not subitizing during visual enumeration. Cognition, 114(2), 293-297. https://doi.org/10.1016/j.cognition.2009.09.013 (cited on page 106)

Mandler, G., \& Shebo, B. J. (1982). Subitizing: An analysis of its component processes. Journal of Experimental Psychology: General, 111(1), 1-22. https://doi.org/10. 1037/0096-3445.111.1.1 (cited on page 101)

Marinova, M., \& Reynvoet, B. (2020). Can you trust your number sense: Distinct processing of numbers and quantities in elementary school children. Journal of Numerical Cognition, 6(3), 304-321. https://doi.org/10.5964/jnc.v6i3.292 (cited on pages 100,104$)$

Marinova, M., Sasanguie, D., \& Reynvoet, B. (2018). Symbolic estrangement or symbolic integration of numerals with quantities: Methodological pitfalls and a possible solution (S. J. Cropper, Editor). PLoS ONE, 13(7), e0200808. https://doi.org/10. 1371/journal.pone.0200808 (cited on page 100)

Marinova, M., Sasanguie, D., \& Reynvoet, B. (2021). Numerals do not need numerosities: Robust evidence for distinct numerical representations for symbolic and nonsymbolic numbers. Psychological Research, 85(2), 1-13. https://doi.org/10.1007/ s00426-019-01286-z (cited on page 100)

Matejko, A. A., \& Ansari, D. (2016). Trajectories of symbolic and nonsymbolic magnitude processing in the first year of formal schooling. PLoS ONE, 11(3). https://doi.org/ 10.1371/journal.pone.0149863 (cited on page 100)

Moyer, R. S., \& Landauer, T. K. (1967). Time required for judgements of numerical inequality. Nature, 215(5109), 1519-1520. https://doi.org/10.1038/2151519a0 (cited on page 98)

Mussolin, C., Nys, J., Content, A., \& Leybaert, J. (2014). Symbolic number abilities predict later approximate number system acuity in preschool children. PLOS ONE, 9(3). https://doi.org/10.1371/journal.pone.0091839 (cited on page 100)

Noël, M. P., \& Rousselle, L. (2011). Developmental changes in the profiles of dyscalculia: An explanation based on a double exact-and-approximate number representation model. Frontiers in Human Neuroscience, 5(November). https://doi.org/10.3389/ fnhum.2011.00165 (cited on page 98)

Odic, D., Le Corre, M., \& Halberda, J. (2015). Children's mappings between number words and the approximate number system. Cognition, 138, 102-121. https : //doi.org/10.1016/j.cognition.2015.01.008 (cited on pages 120, 122) 
Piazza, M. (2010). Neurocognitive start-up tools for symbolic number representations. Trends in Cognitive Science, 14(12), 542-551. https://doi.org/10.1016/j.tics.2010. 09.008 (cited on pages 98, 99)

Pinel, P., Dehaene, S., Rivière, D., \& LeBihan, D. (2001). Modulation of parietal activation by semantic distance in a number comparison task. NeuroImage, 14(5), 1013-1026. https://doi.org/10.1006/nimg.2001.0913 (cited on pages 102, 108, 118)

Reynvoet, B., \& Sasanguie, D. (2016). The symbol grounding problem revisited: A thorough evaluation of the ans mapping account and the proposal of an alternative account based on symbol-symbol associations. Frontiers in Psychology, 7(OCT), 1581. https://doi.org/10.3389/fpsyg.2016.01581 (cited on pages $98,100,102,118)$

Rubinsten, O., Dana, S., Lavro, D., \& Berger, A. (2013). Processing ordinality and quantity: Erp evidence of separate mechanisms. Brain and Cognition, 82(2), 201-212. https://doi.org/https://doi.org/10.1016/j.bandc.2013.04.008 (cited on page 102)

Sasanguie, D., De Smedt, B., Defever, E., \& Reynvoet, B. (2012). Association between basic numerical abilities and mathematics achievement. British Journal of Developmental Psychology, 30(2), 344-357. https://doi.org/10.1111/j.2044-835X.2011.02048.x (cited on page 99)

Sasanguie, D., Defever, E., Maertens, B., \& Reynvoet, B. (2014). The approximate number system is not predictive for symbolic number processing in kindergarteners. Quarterly Journal of Experimental Psychology, 67(2), 271-280. https://doi.org/ 10.1080/17470218.2013.803581 (cited on page 100)

Schneider, M., Beeres, K., Coban, L., Merz, S., Susan Schmidt, S., Stricker, J., \& De Smedt, B. (2017). Associations of non-symbolic and symbolic numerical magnitude processing with mathematical competence: A meta-analysis. Developmental Science, 20(3). https://doi.org/10.1111/desc.12372 (cited on page 100)

Sekuler, R., \& Mierkiewicz, D. (1977). Children's judgments of numerical inequality. Child Development, 48(2), 630. https://doi.org/10.2307/1128664 (cited on page 99)

Sella, F., Berteletti, I., Lucangeli, D., \& Zorzi, M. (2017). Preschool children use space, rather than counting, to infer the numerical magnitude of digits: Evidence for a spatial mapping principle. Cognition, 158, 56-67. https://doi.org/10.1016/j. cognition.2016.10.010 (cited on page 119) 
Sella, F., Lucangeli, D., \& Zorzi, M. (2018). Spatial and verbal routes to number comparison in young children. Frontiers in Psychology, 9(MAY), 776. https://doi.org/10.3389/fpsyg.2018.00776 (cited on page 119)

Sella, F., Lucangeli, D., \& Zorzi, M. (2019). Spatial order relates to the exact numerical magnitude of digits in young children. Journal of Experimental Child Psychology, 178, 385-404. https://doi.org/10.1016/j.jecp.2018.09.001 (cited on page 119)

Šetić Beg, M., Čičko, J., \& Domijan, D. (2021). Symbol grounding of number words in the subitization range. Psychological Research, 85(2), 720-733. https://doi.org/ 10.1007/s00426-019-01265-4 (cited on page 120)

Sullivan, J., \& Barner, D. (2013). How are number words mapped to approximate magnitudes? Quarterly Journal of Experimental Psychology, 66(2), 389-402. https://doi.org/10.1080/17470218.2012.715655 (cited on pages 119, 120)

Sullivan, J., \& Barner, D. (2014). Inference and association in children's early numerical estimation. Child Development, 85(4), 1740-1755. https://doi.org/10.1111/cdev. 12211 (cited on pages 101, 119, 120)

Temple, E., \& Posner, M. I. (1998). Brain mechanisms of quantity are similar in 5-year-old children and adults. Proceedings of the National Academy of Sciences of the United States of America, 95(13), 7836-41. https://doi.org/10.1073/pnas.95.13. 7836 (cited on pages 102, 103, 106, 108, 118)

Uller, C., Carey, S., Huntley-Fenner, G., \& Klatt, L. (1999). What representations might underlie infant numerical knowledge? Cognitive Development, 14(1), 1-36. https: //doi.org/10.1016/S0885-2014(99)80016-1 (cited on page 101)

van den Berg, F. C., de Weerd, P., \& Jonkman, L. M. (2020). Number-related brain potentials are differentially affected by mapping novel symbols on small versus large quantities in a number learning task. Journal of cognitive neuroscience, 32(7), 1263-1275. https://doi.org/10.1162/jocn_a_01546 (cited on pages 102, $105,120,122)$

Wilkey, E. D., \& Ansari, D. (2019). Challenging the neurobiological link between number sense and symbolic numerical abilities. Annals of the New York Academy of Sciences, nyas.14225. https://doi.org/10.1111/nyas.14225 (cited on pages 98-100)

Xenidou-Dervou, I., Molenaar, D., Ansari, D., van der Schoot, M., \& van Lieshout, E. C. (2017). Nonsymbolic and symbolic magnitude comparison skills as longitudinal predictors of mathematical achievement. Learning and Instruction, 50, 1-13. https://doi.org/10.1016/j.learninstruc.2016.11.001 (cited on page 100) 
Xu, F., \& Spelke, E. S. (2000). Large number discrimination in 6-month-old infants. Cognition, 74(1), B1-B11. https://doi.org/10.1016/S0010-0277(99)00066-9 (cited on page 98)

Xu, F., Spelke, E. S., \& Goddard, S. (2005). Number sense in human infants. Developmental Science, 8(1), 88-101. https://doi.org/10.1111/j.1467-7687.2005.00395.x (cited on page 98) 


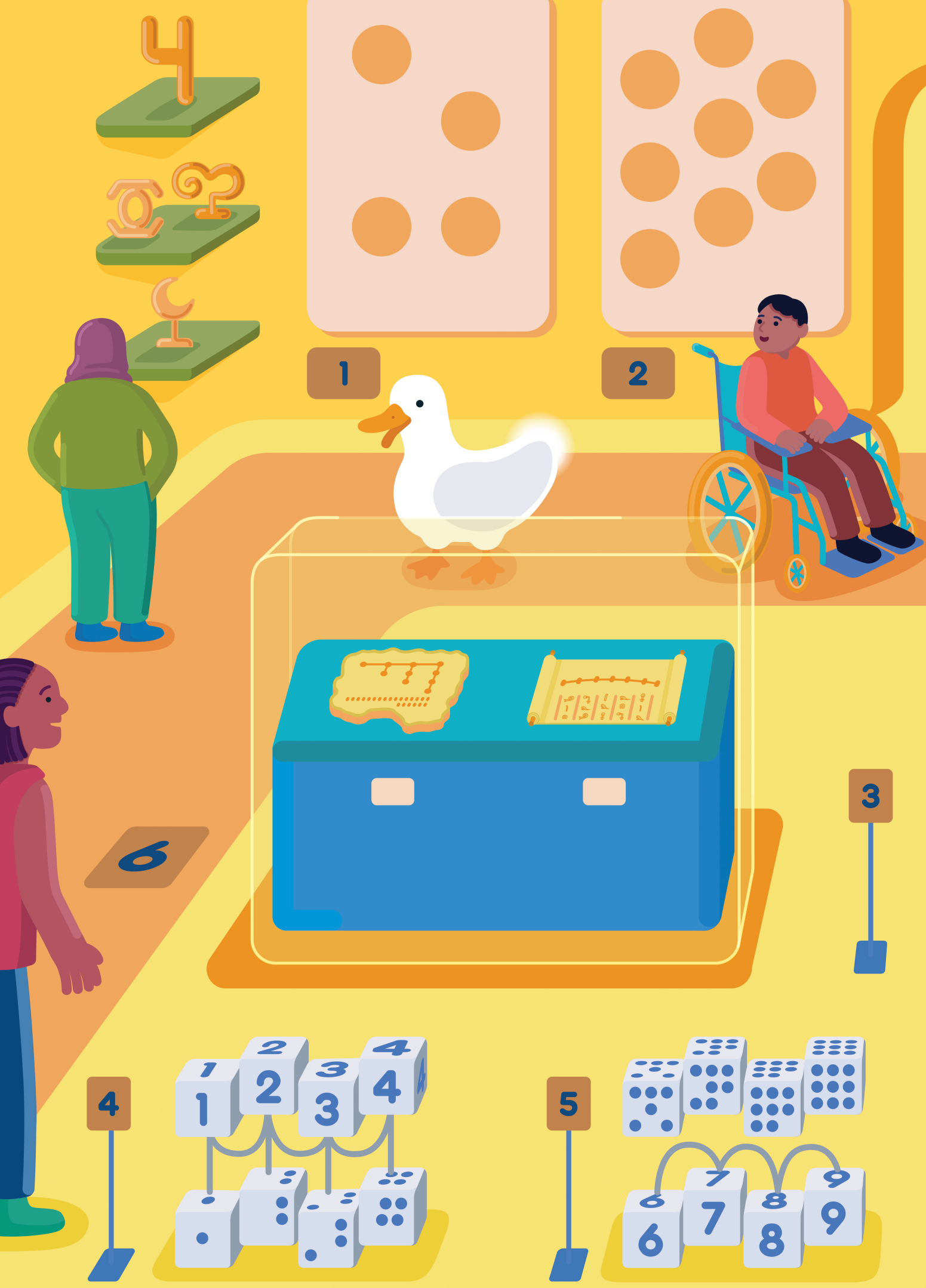




\section{The Symbol Room}

Quantities are all around us; two dogs, six birds, or a dozen eggs. These real-world quantities are called non-symbolic quantities. A lot of animals have some notion of amounts, but humans are the only creatures who also have a symbolic system. In a symbolic system the symbols take on the meaning of an amount, such as 2,6 , or 12. These symbols no longer have a one-to-one connection to the quantity; there's nothing "five" about a 5.

Many animals understand "how much" something is from the day they are born. Without counting it's still possible to "see" how many things there are up to four things (try it with the poster at $\mathbf{1}$ ), when there are more than four things it becomes harder to know the exact amount. The ability to "see" amounts up to four is called "subitizing". However, more than four is too much for our brain to track, and we simply make an estimate that gets less precise the higher the amount (try it yourself with the poster at $\mathbf{2}$ ). For adults this is a bit easier with some quick math tricks.

Representing an amount is done in the brain on a mental number line, where numbers are arranged left-to-right, small-to-large (see 3, the old tablet on the left). A lot of animals and humans can do this naturally. It was thought that any kind of number, be that a symbol (such as 7) or a word (such as 'four') is connected to this number line (see 3 , the papyrus on the right).

New research has taught us that learning numbers is not as straightforward as connecting a symbol to a mental number line. Instead, it seems that the first four symbols are learned through association. By repeatedly hearing "three" combined with three things, children discover that "three" means the abstract quantity and isn't the name of something. This can be seen in $\mathbf{4}$, where the symbols are connected to the dots, but the dots are still the base, that's what children will go back to.

Big numbers are different. These don't have exact representations, only estimations Symbols cannot be connected to an estimation. Instead, numbers larger than four get their meaning through the patterns between symbols. According to the rules, numbers that appear later in the list are larger, and each number is one more than the previous. Children can infer the meaning of a symbol using these rules. The symbols are now only connected to each other and separate from any real-world amounts. We can see this difference at $\mathbf{5}$, where dots are no longer the base.

Putting it all together standing at 6 . A lot of animals can deal with numbers, but only humans came up with convenient symbols. Children must learn those symbols, but they can only learn the first four numbers by slowly connecting them to non-symbolic representations. For larger numbers, the brain cannot "see" an exact amount to connect to the symbol, instead they learn those by generalizing or inferring symbol-to-symbol rules. This is why children take a long time with the first four and then suddenly know all the numbers. It's inferential learning taking over from associative learning. 



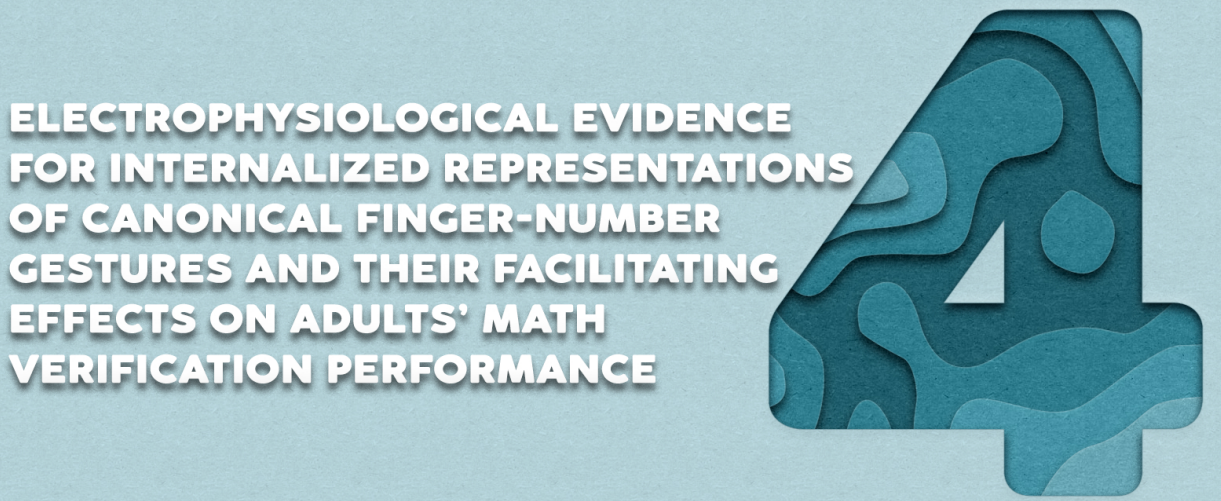

\subsection{Abstract}

Fingers facilitate number learning and arithmetic processing in early childhood. The current study investigated whether images of early-learned, culturally-typical (canonical), finger montring patterns presenting smaller $(2,3,4)$ or larger $(7,8,9)$ quantities still facilitate adults' performance and neural processing in a math verification task. Twenty-eight adults verified solutions to simple addition problems that were shown in the form of canonical or non-canonical finger-number montring patterns while measuring Event Related Potentials (ERPs). Results showed more accurate and faster sum verification when sum solutions were shown by canonical (versus non-canonical) finger patterns. Canonical finger montring patterns 2-4 led to faster responses independent of whether they presented correct or incorrect sum solutions and elicited an enhanced early right-parietal P2p response, whereas canonical configurations 7-9 only facilitated performance in correct sum solution trials without evoking P2p effects. The later central-parietal P3 was enhanced to all canonical finger patterns irrespective of numerical range. These combined results provide behavioral and brain evidence for canonical cardinal finger patterns still having facilitating effects on adults' number processing. They further suggest that finger montring configurations of numbers 2-4 have stronger internalized associations with other magnitude representations, possibly established through their mediating role in the developmental phase in which children acquire the numerical meaning of the first four number symbols.

Corresponding reference: van den Berg, F.C.G., de Weerd, P., \& Jonkman, L. M. (2021). Electrophysiological Evidence for Internalized Representations of Canonical Finger-Number Gestures and their Facilitating Effects on Adults' Math Verification Performance. Nature Scientific Reports, 11(1), 11776. https://doi.org/10.1038/s41598021-91303-2 


\subsection{Introduction}

The very first representations of quantities that children use are fingers, allowing them to communicate numerical information even before having acquired any other numerical representations such as number words or Arabic digits ((Butterworth, 1999; Fuson, 1988). Learning numerical symbols is a complicated and lengthy process, as shown by reports of young children needing 1- 1.5 years to learn the meaning of the first four number words (Wynn, 1990, 1992). One aspect that might make number-symbol learning so difficult is the requirement of making a transition from an analog, non-symbolic representation of numbers (e.g., several items in a set, such as three apples) to a symbolic representation (Le Corre and Carey, 2007). It has been suggested that fingers might mediate this transition in early childhood, acting as a base or aid when memory capacity and strategy use is still immature (Geary, 2004; Gelman and Gallistel, 1978; Soylu et al., 2018). The importance of fingers for number symbol learning is further indicated by findings of finger-number representations facilitating arithmetic processing (Domahs et al., 2008; Klein et al., 2011; Moeller et al., 2011). Although there is some evidence that fingers (in the form of counting) help the early learning of number- symbol relationships (Gelman and Gallistel, 1978), little is known about the cognitive processes enabling this type of learning. Moreover, understanding how finger-number representations might facilitate symbol acquisition is important to address potential symbol learning problems in young children in a later stage. In the present study, we combined behavioral assessments with ERP measurements to investigate whether the potentially privileged role of culture-specific finger-number gestures in early numerosity learning would have left traces in the adult brain.

The underlying idea of the present study is that due to their frequent use in early number learning and daily communication, culture-specific finger-number gestures become internalized and connected to other existing or simultaneously developing non-symbolic or symbolic representations of magnitude or number, rather than only having a function as a temporary external aid during the acquisition of symbolic number knowledge. An important unexplored issue is whether the strength of internalized semantic connections between finger- and other representations of number differs between numbers smaller than five represented by one hand and numbers larger than five represented by two hands. Current models of numerical development and empirical evidence propose that only the first four number symbols are learned by mapping them directly onto their corresponding non-symbolic representation of quantity (Carey and Barner, 2019; Le Corre and Carey, 2007; Reynvoet and Sasanguie, 2016; van den Berg et al., 2020). Once children master 
these first four symbols, they can use symbolic properties such as ordinality, to infer the numerical meaning of number symbols higher than four without referring to their corresponding non-symbolic representations (Carey, 2009; Carey and Barner, 2019). When number gesture representations function as an initial bridge to associate abstract number symbols (e.g., number words) to earlier present non-symbolic representations of magnitude (Gunderson et al., 2015; Jay and Betenson, 2017), stronger functional links between culture-specific finger-number gestures and other representations of number may be present for the first four numbers that are assumed to be learned via direct non-symbolic - symbolic mapping.

Fingers can be used to represent quantities in different ways; one way is during counting when fingers are raised sequentially in a certain order and one finger represents one quantity. Another way in which fingers can be used to communicate and represent quantities is by holding up a hand with three raised fingers simultaneously (as a pattern) to indicate the number three, dubbed 'montring' by Di Luca and Pesenti (2008). Both counting and montring finger configurations are referred to as canonical because they have to be actively acquired and conform to fixed culture-specific habits. Several behavioral studies in adults have reported facilitating effects of canonical finger-number representations on the accuracy and speed of symbolic number retrieval or processing. Di Luca et al. (2006; 2008) demonstrated that the naming of the number represented by early-learned canonical (counting and montring) finger configurations was faster and more accurate than in the case of unfamiliar, non-canonical finger configurations. In a follow-up study, the same authors demonstrated that priming by canonical finger-number montring configurations facilitated the subsequent recognition of other symbolic representations such as Arabic digits in the single-hand range (1-5; Di Luca et al., 2010). These facilitating effects of canonical finger-number configurations have also been shown to extend to arithmetical operations in a study by Badets et al. (2010). In this study, adult participants were presented with a math verification task in which a simple math-sum was given (e.g., $3+4)$, followed by a sum solution that had to be verified as correct or incorrect. Sum solutions were presented in the form of finger-number configurations or as a number of rods. Participants verified sum solutions faster and more accurately when presented in the form of a canonical finger pattern rather than rods, indicating that arithmetic was, to some extent, still embodied in adults. Thus, multiple behavioral studies show facilitating effects of canonical finger-number representations on symbolic number processing in adults, supporting the idea of shared internalized cognitive processes between canonical finger- and other symbolic number representations. 
Various authors have speculated on the processes underlying the number processing benefits of canonical finger montring configurations and suggested that, at least in adults with fully developed symbolic number systems, the early-learned canonical finger configurations have acquired a special status in long-term memory (Della Sala and Anderson, 2012; Di Luca and Pesenti, 2008; Soylu et al., 2019). Specifically, due to the frequent use of fingers during the developmental phase in which children first acquire early numeric and math skills (Gracia-Bafalluy and Noël, 2008; Noël et al., 2005; Soylu et al., 2018), strong semantic associations may have formed between finger-representations of numbers and other non-symbolic (analog quantities) and symbolic (number words, Arabic digits) number representations. As a result, canonical finger representations of numbers may facilitate numeric processes, including number recognition and retrieval. By contrast, the non-familiarity of non-canonical finger-number configurations and the absence of associations with other types of symbolic and non-symbolic number representations, renders number processing more time-consuming and error-prone (Badets et al., 2010; Di Luca et al., 2006; Di Luca et al., 2010; Di Luca and Pesenti, 2008).

However, behavioral measures do not permit the identification of the brain processes responsible for the above reviewed facilitating effects of canonical finger-number configurations on symbolic number processing. Event-related brain potentials can provide information about semantic processing differences between canonical and non-canonical finger-number gestures in the brain. Prior number-processing ERP studies with standard symbolic number stimuli (e.g., Arabic digits) have consistently found that a positivity above the right temporal-occipito-parietal hemisphere peaking around 200-250 milliseconds after stimulus presentation, called the P2p (e.g. the second posterior positivity) by Dehaene (1996), is the first ERP component modulated by numerical semantic processing demands in tasks requiring magnitude judgements (Dehaene, 1996; Libertus et al., 2007; Pinel et al., 2001; Turconi et al., 2004). These studies all used so-called symbolic number comparison tasks in which one has to decide whether a presented number symbol (digit) is larger or smaller in magnitude than another internal reference number, which requires retrieval of the symbols' associated magnitude information (its number semantics) and comparison of the magnitude of the presented symbol with that of the internal reference number. The modulation of the amplitude of the $\mathrm{P} 2 \mathrm{p}$ by this magnitude comparison process, and more specifically by the numerical distance between two to-be-compared number stimuli on the mental number line, has led multiple authors to conclude that the $\mathrm{P} 2 \mathrm{p}$ in symbolic numerical processing tasks, reflects the processing stage in which one has first access to semantic (analog) quantity 
information associated with the number symbols (Dehaene, 1996; Libertus et al., 2007; Pinel et al., 2001; Turconi et al., 2004). In the majority of the above number symbol comparison studies, the P2p numerical distance effects were larger above the right than left posterior hemisphere and Dehaene (1996) accordingly source-localized the response-locked $\mathrm{P} 2 \mathrm{p}$ distance effect in the right parieto-occipito-temporal junction (but see Pinel 2001, who localized the P2p distance effect in bilateral parietal cortex). Further evidence for a functional link between posterior right-lateralized P2p activity and activation of/or access to quantity representations comes from a recent number symbol learning study by van den Berg et al. (2020). In this study, only novel symbols that had acquired numerical meaning by successfully associating them with their corresponding non-symbolic (quantity) representation elicited a $\mathrm{P} 2 \mathrm{p}$ response. Interestingly, two magneto-encephalography (MEG) studies investigated the spatiotemporal processing pattern accompanying the extraction of the meaning or connotation of familiar (versus unfamiliar) hand gestures, for example gestures for 'stop' or 'ok' (Nakamura et al., 2004), or natural versus distorted finger postures (Avikainen et al., 2003). Both studies reported enhanced activity in a $200-380 \mathrm{~ms}$ time window, either in bilateral extra-striate cortex (Avikainen et al., 2003) or in right-hemisphere inferior occipito-temporal, inferior parietal and right STS regions (Nakamura et al., 2004), similar to the timing and location of the $\mathrm{P} 2 \mathrm{p}$ in above discussed number symbol processing studies.

A second endogenous ERP component that has consistently been reported to be modulated in tasks requiring the retrieval and comparison of magnitude codes associated with symbolic and non-symbolic number stimuli, is the centro-parietal P3 that occurs between approximately 300-500 milliseconds after stimulus presentation (Dehaene, 1996; Jiang et al., 2010; Libertus et al., 2007; Paulsen and Neville, 2008; Turconi et al., 2004). Although the literature is less consistent on the functional interpretation of magnitude comparison (e.g., numerical distance) effects on the P3, based on its resemblance to the classical centro-parietal P3 (Polich, 2007), it has been linked to later stimulus evaluation/categorization processes, with higher amplitudes reflecting easier categorization and/or higher response confidence (Dehaene, 1996; Libertus et al., 2007), likely due to better memory representations. 
To the best of our knowledge, only two recent studies investigated ERP responses to canonical finger- number stimuli using different symbolic number tasks. Proverbio and Carminati (2019) presented adult participants with a math verification task in which sum solutions shown as Arabic numerals had to be verified. Arabic numerals were flanked by to-be-ignored, task-irrelevant, canonical finger-counting configurations of numbers 0-10 showing the same (congruent) or a different (incongruent) number than indicated by the Arabic numeral. Results showed that congruent finger-number configurations interfered with the processing of the Arabic numeral, yielding lower accuracy, which is in contrast with earlier reported facilitating effects of canonical finger-number patterns on math verification (Badets et al., 2010). Proverbio and Carminati (2019) focused their ERP analyses on frontal and central electrodes and did thus not report on effects of finger-stimuli on posterior $\mathrm{P} 2 \mathrm{p}$ or $\mathrm{P} 3$ components related to semantic processing of number stimuli in the prior number processing literature. Furthermore, the study only included canonical (not non-canonical) finger-number stimuli and the task did not require explicit processing of the numerical meaning of the finger patterns. In another study by Soylu et al. (2019), ERPs were measured in response to single hand stimuli representing numbers 1-4 showing counting, montring, and non-canonical finger-number configurations. The participant's task was to decide whether the quantity shown by the fingers was the same or different from a later appearing Arabic digit, requiring active retrieval of the number represented by the fingers. The authors reported that compared to both counting and non-canonical finger configurations, montring configurations were processed faster and more accurately which went along with enhanced early perceptual P1/N1 responses. The amplitude of the later endogenous centro-parietal P3 component, measured in a 250-500 ms time window, was however enhanced to the same extent by both canonical (counting and montring) finger stimuli. Based on these findings, the authors concluded that only finger montring patterns showed facilitated processing and elicited higher early perceptual/attentional processing (as indexed by P1 and N1), whereas, in line with earlier suggestions by Di Luca et al. (2010), the higher P3 response to both canonical (counting and montring) stimuli was interpreted as signifying their retrieval from memory. These findings suggest that canonical finger-number montring patterns are easier to enumerate because of the faster automatic activation/retrieval of their associated numeric representations from long-term memory. 
These above discussed studies were either limited to studying canonical finger-number representations of smaller numbers 1-4 (Di Luca et al., 2010; Soylu et al., 2019) or did use a larger range up to 10 (Proverbio and Carminati, 2019), but did not include numerical range as a factor in their analyses. As explained above, making such a distinction is important because of propositions that fingers may scaffold number symbol acquisition by facilitating the process of mapping abstract number symbols (digits, words) onto nonsymbolic representations of magnitude. Because only the first four number symbols are suggested to be learned via such one-to-one symbolic - non-symbolic mapping (Carey and Barner, 2019; Le Corre and Carey, 2007; Reynvoet and Sasanguie, 2016; van den Berg et al., 2020), associations between canonical finger-montring patterns and other representations of number might be stronger for the small (1-4) than for the larger (6-9) number range.

The aim of the present study was two-fold: 1) to investigate the semantic processing of canonical finger-number montring gestures (versus non-canonical gestures) and their facilitating effects on number magnitude comparison in the adult brain by, besides on behavior, also investigating effects on the P2p and P3-ERP components that have in earlier studies been linked to early and late stages of number semantic processing (see above) and 2) to compare the semantic processing of canonical finger gestures showing small numbers 2-4 represented on one hand with those showing larger numbers 7-9 represented on two hands. To investigate this, we used a simple math verification task based on Badets et al. (2010) and Proverbio \& Carminati (Proverbio and Carminati, 2019), in which participants were required to compute a sum by adding two visually presented one-digit numbers, and had to verify whether a subsequently presented canonical or non-canonical finger-number pattern (numerical ranges 2-4 or 7-9) showed the correct or incorrect sum solution. With respect to the first aim, we expect that if canonical finger-number gestures are indeed stored in long-term memory and have gained associations with other numeric (quantity) representations, we will find a main effect of canonicity on the amplitude of the right-lateralized P2p component (Dehaene, 1996; Libertus et al., 2007; Turconi et al., 2004). More specifically, we hypothesize that canonical finger-number gestures will elicit faster math verification responses and higher (right-lateralized) P2p amplitudes than noncanonical finger-number gestures (canonicity*hemisphere interaction). We further expect that canonical finger-number gestures will elicit higher amplitudes of the later centroparietal P3-ERP component than non-canonical finger-number gestures (main canonicity effect), evidencing their easier, or less ambiguous classification due to their storage in and retrieval from long term memory (Dickson and Wicha, 2019; Soylu et al., 2019). With 
respect to the second aim; if small canonical finger-number gestures (2-4) have developed stronger semantic numeric associations than canonical finger patterns gesturing larger numbers 7-9, we expect to find canonicity*numerical range interaction effects on reaction time, P2p (including factor hemisphere) and P3 amplitude, with stronger canonicity effects (faster comparison reaction time, higher right-posterior P2p amplitude and higher centroparietal P3 amplitude to canonical than non-canonical) for finger gestures showing small sum solutions (2-4) than for gestures showing larger sum solutions (7-9). Finally, in math verification paradigms correct sum solution trials standardly elicit better performance and higher P3 amplitude than trials showing an incorrect sum solution (Dickson and Wicha, 2019; Proverbio and Carminati, 2019). To be able to distinguish between facilitating effects of canonicity and numerical range of the finger patterns and effects of presented sum solutions being correct or incorrect, all above analyses will also include the trial-type factor correct/incorrect sum solutions. 


\subsection{Method}

\section{Participants}

Participants were thirty-four young adults recruited from the student population at Maastricht University, The Netherlands. One participant was excluded due to technical difficulties with the EEG recording, and five other participants were excluded because of below chance performance on the task. All reported analyses included the remaining 28 participants (mean age: $21 \mathrm{yrs} 4 \mathrm{mo}$ [SD 1yrs 6mo]; 2 left-handed; 8 males). All adults provided written informed consent after being informed about the study. Participants were rewarded with university course credits for their participation. The current study was approved by the local Ethical Review Committee Psychology and Neuroscience of Maastricht University, The Netherlands (ERCPN_RP2027_2019_34). All procedures were in accordance with the Declaration of Helsinki.

\section{Procedure}

Testing took place at the dedicated EEG labs in a sound-proof booth and started with an explanation of the procedure, followed by attachment of the electrodes. Participants first performed another task, followed by the current Math verification task, and during both tasks their EEG was measured. Before the real math verification task started, all participants practiced the task until a performance criterion of $70-80 \%$ correct responses was reached to be sure that they understood the task instructions and were able to perform the task. Instructions were to respond as fast and as accurately as possible, and participants were asked to minimize eye blinks and (eye) movements during the tasks.

\section{Math verification task}

In the math verification task, participants were presented with simple addition problems consisting of one-digit Arabic numerals (e.g., $2+4$ ), followed by a solution shown in the form of canonical or non-canonical hand-finger configurations (see Figure 5 for an example of a complete trial including timing information). The sums were chosen in such a way that sum solutions shown by the hands fell into the small (2-4) or large (7-9) number range. In $50 \%$ of the trials presented sum solutions were correct and in the other $50 \%$ incorrect and the participants' task was to press the left button on a Cedrus RB-844 button box when the sum was correct and the right button in case it was incorrect. 
The math verification task consisted of a total of 288 trials (presented in three blocks of 96 trials), of which half of the finger-number montring configurations represented an incorrect solution and the other half a correct solution. Further, sum solutions represented by the hands fell in either the small number range $(2,3$, or 4$)$ or the large number range $(7,8$, or 9$)$, and numerical range was balanced across the 288 trials. Across all trials, each solution was presented 24 times by non-canonical and 24 times by canonical hands. Stimulus presentation was quasi-random, with the restrictions of identical sum solutions never being presented directly after each other, and one type of hand stimulus (canonical/non-canonical) never shown more than four times in a row. In half of the incorrect trials the number represented by the hands had a distance of 1 from the correct Solution, while the other half had a distance of 2. All stimuli were presented on grey backgrounds inside white boxes at the center of a 19-inch monitor with a viewing distance of $57 \mathrm{~cm}$ using the PsychoPy python package (Peirce et al., 2019).

\section{Selection of finger pattern stimuli}

The selection of the non-canonical and canonical montring stimuli included in the present study was based on a small pilot questionnaire study with $\mathrm{N}=14$ students that were presented with a row of four pictures showing different finger patterns representing the same number. Two of the four presented patterns were different non-canonical patterns and the other two were the two possible canonical patterns with adjacently raised fingers but one canonical pattern including the thumb (often called 'counting' pattern) and the other canonical pattern without the thumb (often called 'montring' pattern). The students were asked to rate the four finger patterns on the likelihood that they would use them to communicate a number to someone else (scale 1- 4, with 1 highest likelihood and 4 lowest likelihood). For each of the included numbers 2, 3, and 4, out of the two noncanonical finger patterns we chose the pattern that was rated as most unlikely being used for communicating the number. Of the two canonical patterns we (for each number) chose the canonical pattern that was indicated by the majority as the most likely used way to communicate the number (although both canonical patterns did not differ much in rating but both differed strongly in rating from both non-canonical patterns). This resulted in the non-canonical and canonical finger patterns shown in Figure 5 (note that the same finger patterns for 2, 3 and 4 were used for higher numbers $7,8,9$, only accompanied by a full-hand indicating 5, whereas in the case of 2, 3, 4 a second hand was also shown but with a closed fist representing zero). 

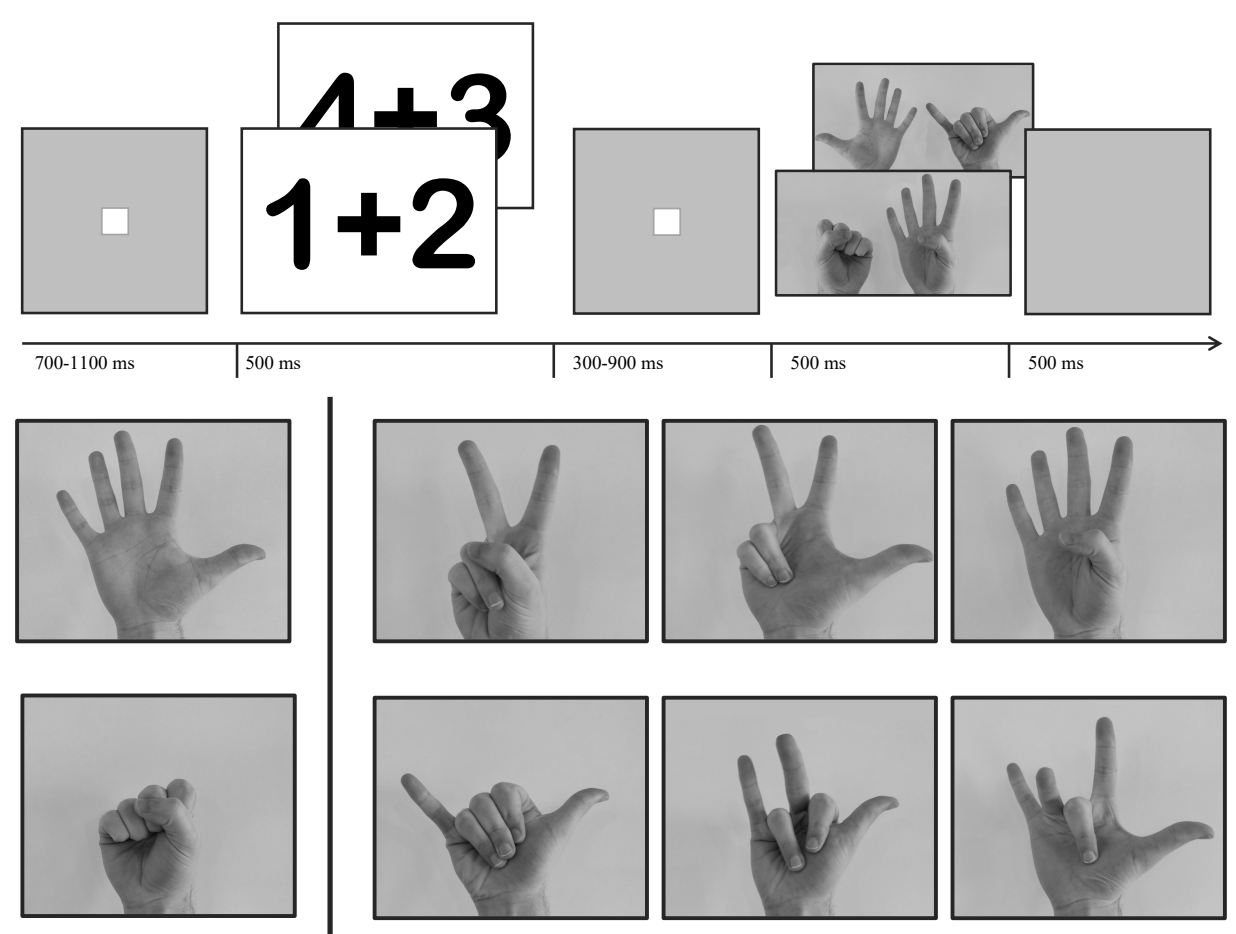

Figure 4.1: The Math Task (top) showing two examples of addition problems presented in Arabic Numerals, followed by a variable interval (between 300 and $900 \mathrm{~ms}$ ), followed by a problem solution presented by the hands (foreground: incorrect problem solution represented by a canonical finger configuration; background: correct problem solution represented by a non-canonical finger representation). The montring finger stimuli (bottom) were composed of canonical (top row) or non-canonical (bottom row) patterns combined with an open (top row left) or closed hand (bottom row left). Task stimuli were drawn hands (see Petrilla, 2012), but due to copyright considerations photos are shown in this figure. 


\section{EEG/ERP acquisition and analyses}

Electro-Encephalographic (EEG) measurements took place in a lab at the University. EEG data were recorded using an elastic EEG-electrode cap (EasyCap; Nellcor-Puritan Bennet, Hayward, CA) with a 38 tin electrode set-up (FP1, FP2, F7, F3, Fz, F4, F8, FC5, FC1, FCZ, FC2, FC6, T7, C3, CZ, C4, T8, CP5, CP1, CPZ, CP2, CP6, P7, P3, PZ, P4, P8, PO7, O1, OZ, O2, A2, AFZ, A1). The data were filtered online at 0.01-225 Hz and continuously sampled at a rate of $500 \mathrm{~Hz}$ using a BrainAmp amplifier system and software (BrainVision Analyzer, Vers. 2.2.0, Brain Products GmbH, Gilching, Germany "BrainVision Analyzer (Vers. 2.2.0)[Software]", 2019). The AFz electrode served as ground, and the left mastoid (A1) acted as the online reference; the right mastoid (A2) was included as an extra active electrode. Horizontal and vertical EOG was measured by electrodes placed on respectively the outer canthus of each eye and above/below the left orbit. All electrode impedances were kept below 5-10 kOhm and were frequently checked during testing.

For offline analyses of the EEG data, Matlab2019a/EEGlab2019/ERPLABv7.0.0 software was used (Delorme and Makeig, 2004; Lopez-Calderon and Luck, 2014; MATLAB, 2019). The data was first resampled to $250 \mathrm{~Hz}$ and re-referenced to the average signal. Next, a band-pass filter of $0.1 \mathrm{~Hz}$ to $70 \mathrm{~Hz}$ was applied to the data before execution of Independent Component Analysis (ICA) for removal of horizontal eye movements and blinks. The ocular artifact-free data was filtered using a $30 \mathrm{~Hz}$ Low-pass filter, after which the data was epoched (based on the dot-quantity and symbol-quantity event-codes, excluding epochs with incorrect responses) into $300 \mathrm{~ms}$ pre-stimulus and $1000 \mathrm{~ms}$ post-stimulus windows. Baseline correction was performed using the pre-stimulus interval. Next, an automatic artifact detection procedure was applied to the remaining epochs (only at electrodes $\mathrm{P} 3, \mathrm{P} 4$, and the average channel of $\mathrm{Cz}$ and $\mathrm{CPz}$ that were respectively included in the P2p and P3 analyses), rejecting trials with activity exceeding a $\pm 50 \mu \mathrm{V}$. Participants were only included in the subsequent analyses if at least $50 \%$ of total trials remained for averaging (after removal of incorrectly responded and artifact trials). This led to the removal of three participants based on accuracy and two participants based on EEG artifacts, as mentioned in the participant section. Of the remaining 28 participants, an average of $27.13 \%(27.21 \%$ [9.72-44.44\%] due to incorrect responses, and $1.17 \%$ [0-18.97\%] due to artifacts) of the total number of trials were rejected. 
The choice of electrodes and time-windows for the P2p and P3 analyses was based on 1) the previous number processing ERP literature (see introduction) in which similar number processing (comparison) tasks and/or similar finger stimuli were used and 2) on visual inspection of the grand average ERP signals and topography maps in the different conditions (see Figures 4.3 and 4.4 for grand average ERPs and topo-maps). This led to the analyses being focused on an early positivity (P2p) in an early 220-310 ms time window at left and right parietal (P3 and P4; Avikainen et al., 2003; Dehaene, 1996; Libertus et al., 2007; Nakamura et al., 2004; Pinel et al., 2001; Turconi et al., 2004; van den Berg et al., 2020) electrodes, and a later positivity (P3) in a $280-550 \mathrm{~ms}$ window at $\mathrm{CPz}$ and $\mathrm{Cz}$ electrodes (Dehaene, 1996; Jiang et al., 2010; Libertus et al., 2007; Paulsen and Neville, 2008; Polich, 2007; Soylu et al., 2019; Turconi et al., 2004). Mean amplitude values within these time windows were entered in the statistical analyses.

\section{Statistical Analysis}

For dependent measures reaction time (RT), P2p amplitude and P3 amplitude, Linear Mixed-effects models were constructed in IBM SPSS 27 (Analytics, 2021). For the accuracy data, Generalized Estimation Equation (GEE) models were constructed because of severe violation of normality. All models included within-subject factors Canonicity (2 levels: canonical and non-canonical), sum Solution (2 levels: correct and incorrect sum solution trials), and numerical Range (2 levels: low numerical range [numbers 2, 3, 4] and high numerical range [numbers $7,8,9]$ ). The $\mathrm{P} 2 \mathrm{p}$ amplitude analysis included an extra factor Hemisphere (2 levels: left and right).

Assumptions were checked using a compound Symmetry covariance structure, removing single observations (single data-points) as outliers if residuals exceeded three standard deviations. No observations were excluded for the ERP analysis. For the behavioral data, a single outlier was removed for RT, while six observations were removed from the accuracy analysis. Assumptions of normality and homoscedasticity were met in all analyses. For dependent variables RT and ERP (P2p and P3) amplitude, various covariance matrices were compared using AIC to determine the best fitting model. For the RT and P2p-ERP models, the Toeplitz structure showed the best and more parsimonious fit, whereas the P3 data fitted best with a Compound Symmetry structure. All models were subsequently reduced by removing non-significant four-way and three-way interactions. Non-significant two-way interactions were purposely kept in the model; removing them did not change any of the conclusions. 


\subsection{Results}

All analyses included within-subject factors Canonicity (2 levels: canonical and nonnanonical), sum Solution (2 levels: correct and incorrect sum solutions), and numerical Range (2 levels: low [numbers 2, 3, 4] and high [numbers 7, 8, 9]). Only the P2p amplitude analysis included an extra factor Hemisphere (2 levels: left and right).

\section{Behavioral results}

RT. The analysis yielded significant main effects of Canonicity $(F[1,28.86]=79.50, p<$ $.001)$, Solution $(F[1,51.96]=51.59, p<.001)$, and Range $(F[1,24.98]=97.23, p<.001)$, and a three-way interaction between these factors $(F[1,37.26]=6.29, p=.017$ : see Figure 4.2 and Table 5.1). The three-way interaction was further explored by splitting up on the Solution factor. For correct sum solution trials the Canonicity * Range interaction was not significant $(F[1,24.40]=2.09, p=.161)$, but main effects of Canonicity $\left(\mathrm{M}_{\text {Diff }}=\right.$ $\left.-51.88 \mathrm{~ms} ; t(31.27)_{\mathrm{NCan}-\mathrm{Can}}=9.93, p<.001\right)$ and Range $\left(\mathrm{M}_{\text {Diff }}=53.18 \mathrm{~ms} ;\right.$ $\left.t(24.84)_{\text {High-Low }}=6.16, p<.001\right)$ respectively showed that sum verification time was significantly faster for canonical than non-canonical finger patterns (for both patterns 2-4 and 6-9) and decisions were overall faster for small than large sum solutions (irrespective of their canonicity). For incorrect sum solution trials, a significant Canonicity*Range interaction was found $(F[1,32.51]=22.08, p<.001)$, and further (Bonferroni corrected) pairwise comparisons showed a significant canonicity effect only for finger patterns showing numbers 2-4 (faster verification times for canonical; $\mathrm{M}_{\text {Diff }}=34.10 \mathrm{~ms}$; $\left.t(50.23)_{\mathrm{NCan}-\mathrm{Can}}=5.05, p<.001\right)$, whereas the canonicity effect for finger patterns $6-9$ was non-significant $\left(\mathrm{M}_{\text {Diff }}=-5.74 \mathrm{~ms} ; t(50.23)_{\mathrm{NCan}-\mathrm{Can}}=-0.85, p=.397\right)$. Comparing the range effect per canonicity-level showed a larger range effect (slower responses for the high range $)$ for Incorrect Canonical $\left(\mathrm{M}_{\text {Diff }}=87.78 \mathrm{~ms} ; t(49.69)_{\text {High-Low }}=11.24, p<.001\right)$ compared to Incorrect Non-Canonical $\left(\mathrm{M}_{\text {Diff }}=47.94 \mathrm{~ms} ; t(49.69)_{\text {High-Low }}=6.14, p<\right.$ $.001)$ trials. These further tests thus indicate that the lack of a canonicity effect for finger patterns 6-9 on incorrect trials is due to a relatively large increase in reaction time for canonical finger patterns 6-9 signifying an incorrect solution. 


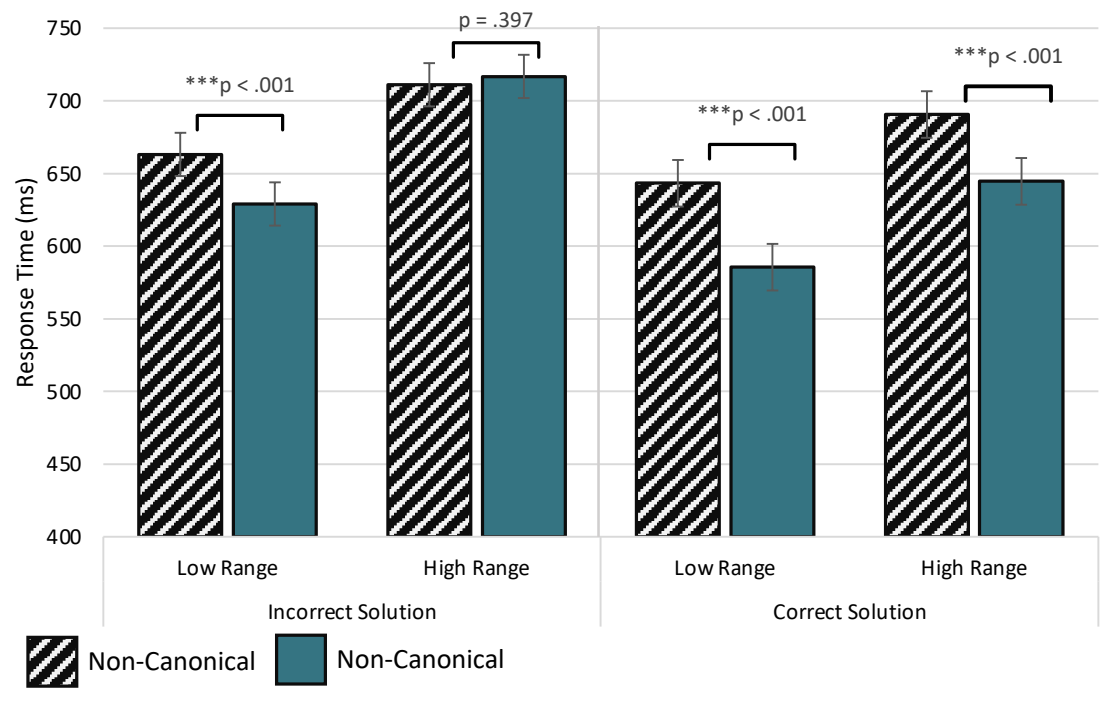

Figure 4.2: Canonicity effects on Reaction Time for the Low and High numerical ranges and for Incorrect and Correct Solution trials (three-way Canonicity*Numerical Range* Solution interaction).

Accuracy. The analysis of accuracy only showed significant main effects for Canonicity $\left(\chi_{1}^{2}=9.07, p=.003\right)$ and Range $\left(\chi_{1}^{2}=6.16, p=.013\right.$; see table 5.1), none of the other terms reached significance (all p-values $>.225$ ). Accuracy was higher on canonical than non-canonical trials $\left(\mathrm{M}_{\text {Diff }}=2.75 \% ; t(1)_{\text {Can-NCan }}=3.10, p=.002\right)$ and for finger-number configurations in the low (2-4) compared to the higher (6-9) numerical range $\left(\mathrm{M}_{\text {Diff }}=5.78 \% ; t(1)_{\text {Low-High }}=2.56, p=.010\right)$.

\section{ERP results}

\section{Posterior Positivity between 220-310ms (P2p)}

The Grand average ERPs and topomaps presenting P2 effects are shown in Figure 4.3. The analysis of the first positivity (P2p) at left and right parietal electrodes showed main effects of Canonicity $(F[1,36.67]=4.80, p=.035)$, Range $(F[1,53.61]=40.11, p<$ $.001)$, and Hemisphere $(F[1,26.77]=5.34, p=.029)$. Additionally two-way interactions between Canonicity*Solution $(F[1,59.02]=10.68, p=.002)$, Range*Solution $(F[1,59.94]$ $=5.92, p=.018$ ), showing a canonicity effect (larger amplitude for canonical trials) on trials where fingers showed incorrect sum solutions $\left(\mathrm{M}_{\text {Diff }}=0.46 \mu \mathrm{V} ; t(72.01)_{\mathrm{Can}-\mathrm{NCan}}\right.$ $=3.85, p<.001)$ but not when they showed correct sum solutions $\left(\mathrm{M}_{\text {Diff }}=0.08 \mu \mathrm{V}\right.$; $\left.t(72.01)_{\text {Can-NCan }}=.70, p=.485\right)$. The range effect (larger amplitude for the low range) 
was larger for correct sum solution trials $\left(\mathrm{M}_{\text {Diff }}=0.88 \mu \mathrm{V} ; t(111.42)_{\text {Low-High }}=6.34, p<\right.$ $.001)$ than for incorrect sum solution trials $\left(\mathrm{M}_{\text {Diff }}=0.43 \mu \mathrm{V} ; t(11.42)_{\text {Can-NCan }}=3.08, p=\right.$ $.003)$. Finally, the analyses yielded a two-way Range*Hemisphere $(F[1,53.40]=22.91$, $p<.001)$, and a three-way Canonicity, Range, and Hemisphere $(F[1,55.06]=4.93, p=$ .031) interaction. The three-way interaction was further explored by splitting up on the Hemisphere factor. A significant Canonicity*Range interaction was found at P4 above the right parietal cortex $(F[1,43.24]=4.43, p=.041)$, but the interaction was strongly non-significant at the $\mathrm{P} 3$ electrode above left parietal cortex $(F[1,44.70]=0.22, p=.639$; see top panel Figure 4.5A). Further (Bonferroni corrected) pairwise comparisons at the right hemisphere (P4) electrode showed a significant canonicity effect signified by higher amplitudes to canonical than non-canonical hands for the low (2-4) number range $\left(\mathrm{M}_{\text {Diff }}\right.$ $\left.=0.45 \mu \mathrm{V} ; t(77.07)_{\text {Can-NCan }}=2.75, p=.007\right)$, whereas the canonicity effect for the high range was highly non-significant $\left(\mathrm{M}_{\text {Diff }}=-0.02 \mu \mathrm{V} ; t(77.07)_{\text {Can-NCan }}=-0.10, p=.919\right)$. As was expected on the basis of the non-significant interaction, no significant canonicity effects on ERP amplitude were found above the left parietal hemisphere (electrode P3) in low or high number ranges (Low: $\mathrm{M}_{\text {Diff }}=0.11 \mu \mathrm{V} ; t(81.65)_{\text {Can-NCan }}=0.79, p=.431$; High: $\left.\mathrm{M}_{\text {Diff }}=0.20 \mu \mathrm{V} ; t(81.65)_{\text {Can-NCan }}=1.46, p=.147\right)$.

\section{Positivity between 280-550ms (P3)}

The Grand average ERPs and topomaps presenting P2 effects are shown in Figure 4.4. In this time window, the topography maps show a clear positivity (P3) with maximum amplitude above midline $\mathrm{Cz}$ and $\mathrm{CPz}$ electrodes and the $\mathrm{P} 3$ amplitude averaged across $\mathrm{Cz}$ and $\mathrm{CPz}$ was entered in the analysis. The analysis yielded main effects for Canonicity $(F[1,181.99]=19.86, p<.001)$, Range $(F[1,181.99]=36.19, p<.001)$, and Solution $(F[1,181.99]=32.01, p<.001)$, and a two-way Range*Solution interaction $(F[1,181.99]$ $=4.82, p=.029)$. Amplitude was significantly larger for Canonical finger-number representations $\left(\mathrm{M}_{\text {Diff }}=0.46 \mu \mathrm{V} ; t(118.99)_{\text {Can-NCan }}=4.46, p<.001\right)$. Similarly, correct solutions elicited higher P3 amplitude compared to incorrect solutions, but this difference was larger in the lower numerical range $\left(\mathrm{M}_{\text {Diff }}=0.81 \mu \mathrm{V} ; t(181.98)_{\text {Correct-Incorrect }}=5.57\right.$, $p<.001$; see bottom panel Figure 4.5B) than the higher range $\left(\mathrm{M}_{\text {Diff }}=0.36 \mu \mathrm{V}\right.$; $\left.t(181.99)_{\text {Correct-Incorrect }}=2.44, p=.016\right)$. 

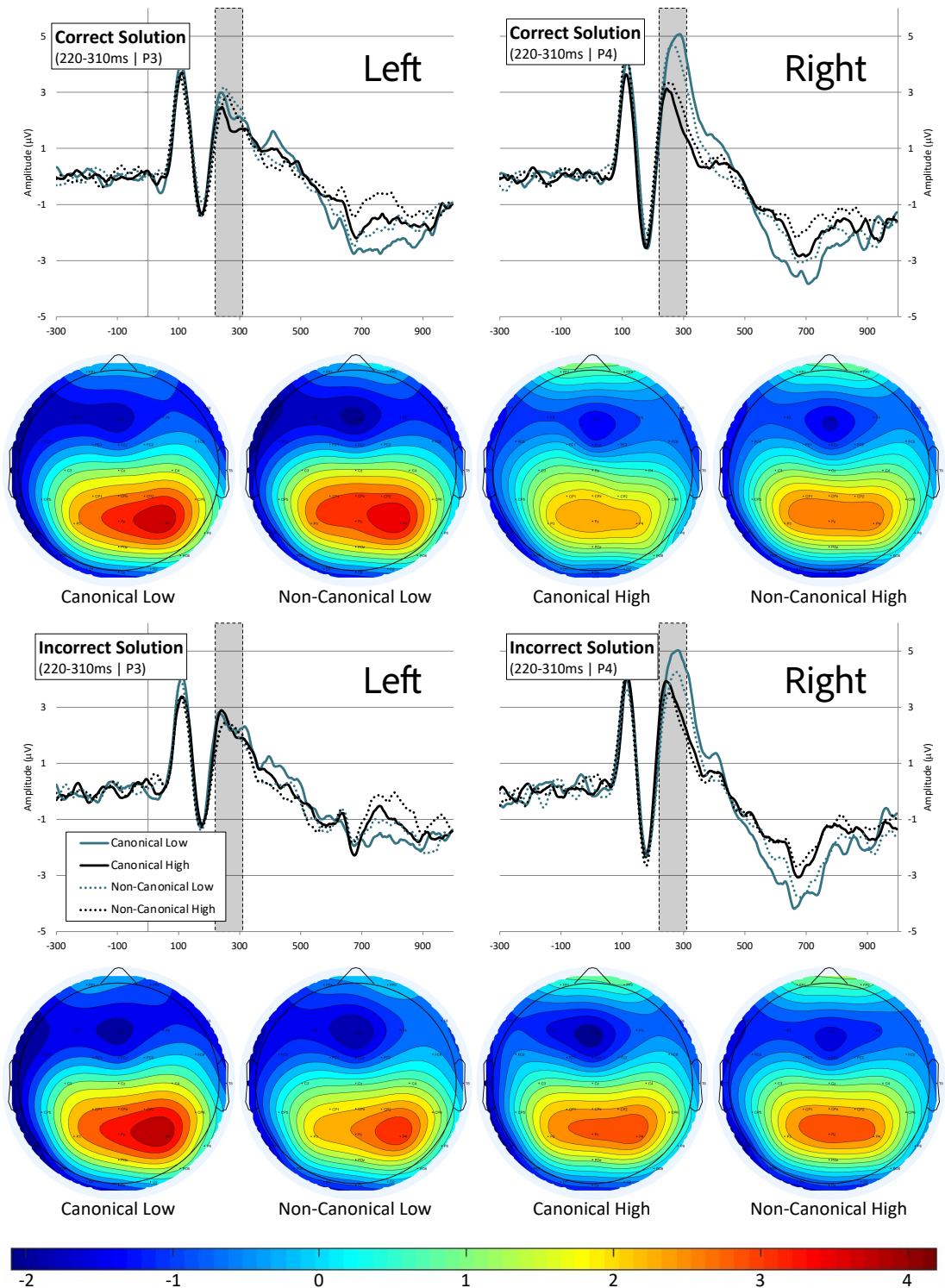

Figure 4.3: Grand average ERP's at the left parietal (P3) electrode (left panel) and the right parietal (P4) electrode (right panel) with the $\mathrm{P} 2 \mathrm{p}$ time window used in the analysis indicated by the grey bar. Topography plots for the 220-310 ms (P2p) time-window are shown separately for correct (top panel) and incorrect (bottom panel) sum solution trials in the four Canonicity-Range categories: Canonical-Low, Non-Canonical-Low, Canonical-High, Non-Canonical-High. The ERP plots show the grand average ERPs and topo-plots in all conditions of the experiment for purposes of evaluation of ERP quality and choice of windows and electrodes for analyses (see: Keil et al., 2014). For visualization of the specific interaction effects found on P2p and P3 amplitude we refer the reader to Figure 4A. Images are created by the first author using ERPlab software (Lopez-Calderon and Luck, 2014) 

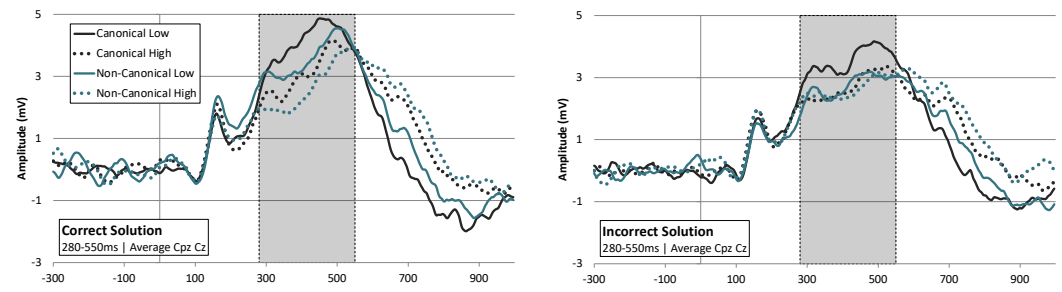

\section{Correct Solution}
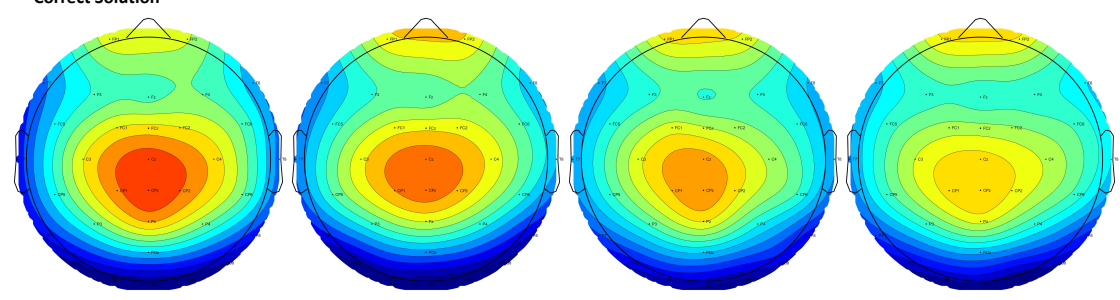

Incorrect Solution

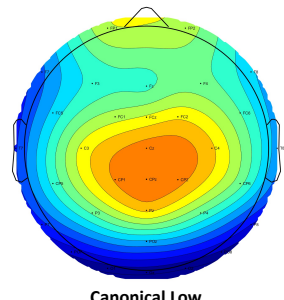

Canonical Low

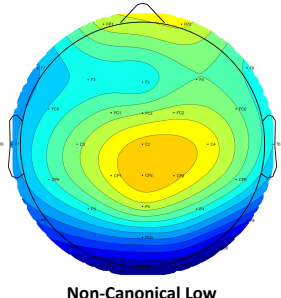

$-1$
Non-Canonical Low 0

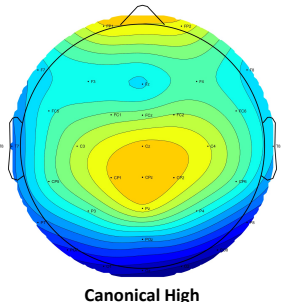

Canonical High 2

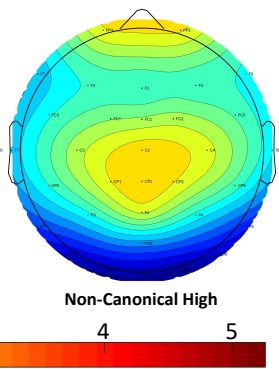

Figure 4.4: Grand average ERP's (averaged over $\mathrm{CPz}$ and $\mathrm{Cz}$ electrodes) with the $\mathrm{P} 3$ time window indicated by the grey bar; left and right ERP graphs respectively show the grand average ERPs on correct and incorrect sum solution trials. Topography plots for the $280-550 \mathrm{~ms}$ (P3) time-window for correct and incorrect sum solution trials in the four Canonicity-Range categories: Canonical-Low, Non-Canonical-Low, Canonical-High, Non-Canonical-High. The ERP plots show the grand average ERPs and topo-plots in all conditions of the experiment for purposes of evaluation of ERP quality and choice of windows and electrodes for analyses (see: Keil et al., 2014). For visualization of the specific interaction effects found on P2p and P3 amplitude we refer the reader to Figure 4B. Images were created by the first author using ERPlab software (Lopez-Calderon and Luck, 2014) 


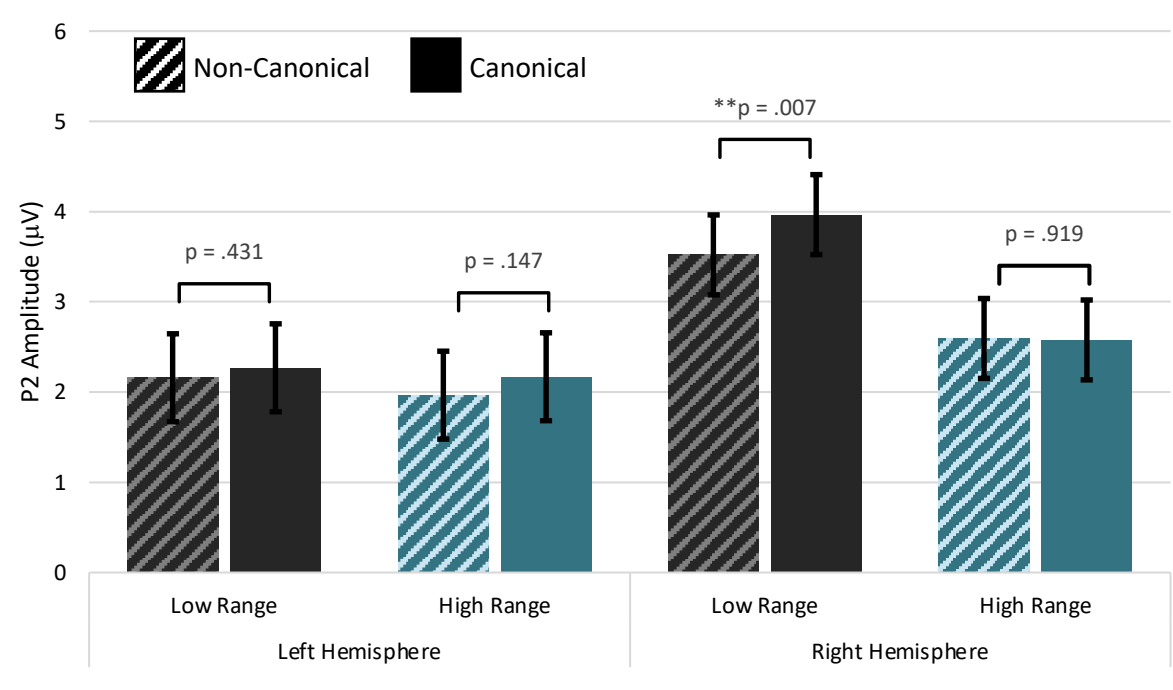

6

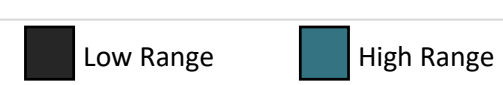

5

$$
p<.001
$$

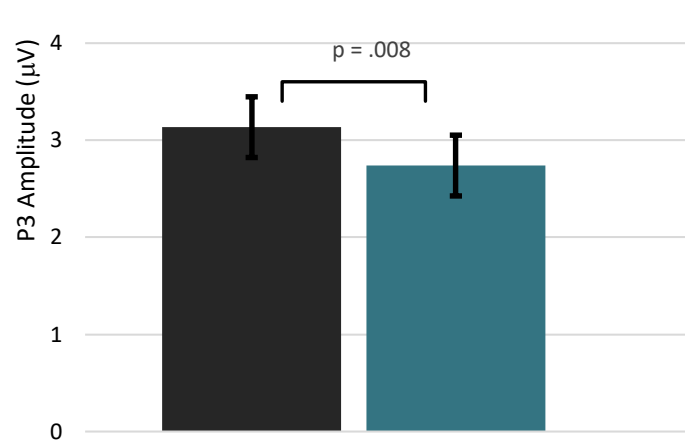

Incorrect Solution

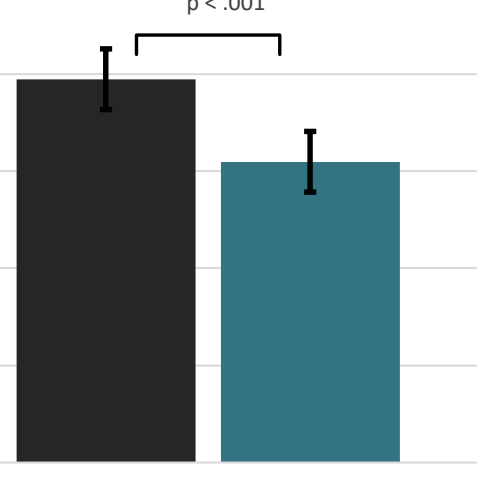

Correct Solution

Figure 4.5: Top: Mean P2p amplitude (averaged across the 220-310 ms time window) at left and right hemisphere parietal (P3 and P4) electrodes in the four Canonicity-Range conditions: Canonical-Low, Non-Canonical-Low, Canonical-High, Non-Canonical-High. The figure shows the three-way interaction between Canonicity, Range and Hemisphere with a statistically significant canonicity effect on P2p amplitude occurring only in response to finger-number configurations showing small numerosities 2-4 above the right parietal hemisphere. Bottom: Mean P3 amplitude averaged across CPz and Cz electrodes in the four Solution-Range categories: Incorrect-Low, Correct-Low, Incorrect-High, Correct-High, showing the Solution * Range interaction with a larger numerical range effect on P3 amplitude (enhanced activation in response to hands showing small numbers 2-4) on trials with correct compared to incorrect sum solutions. 


\section{Correlations between ERPs (P2 and P3) and Reaction Time}

To investigate possible relations between the canonicity effects on reaction time and P2p and P3 amplitude, we performed two exploratory post-hoc correlation analyses: one for the P2p and one for the P3. The $\alpha$-level corrected for multiple comparisons is $\mathrm{p}<.025$ for the $\mathrm{P} 2 \mathrm{p}$ analysis and $\mathrm{P}<.0125$ for the $\mathrm{P} 3$ correlation analyses. Because the canonicity effect on the P2p was only significant at the right parietal electrode and only for finger montring patterns showing small numbers $2-4$, a zero-order correlation was computed between right-parietal P2p amplitude and RT in response to canonical finger montring patterns 2-4 (averaged over correct and incorrect sum solution trials because for patterns 2-4 canonicity effects on P2p and RT were present in both types of trials). The zero order correlation between right-parietal P2p amplitude and RT in response to canonical finger patterns showing numbers 2-4 was not significant $(r[26]=-.15, p=.450)$, and this did not change after controlling for right-parietal P2p amplitude and reaction time in response to non-canonical finger patterns $2-4\left(r_{\text {partial }}[24]=.18, p=.380\right)$.

Next, similar zero-order and partial correlations (the latter controlling for P3 and RT responses to non-canonical finger patterns) were computed between mean P3 amplitude and mean RT averaged across all 6 (2-4 and 7-9) canonical finger montring patterns. This was based on the finding of a main canonicity effect on P3 (and no interaction with numerical range). Correct sum solution trials did show a significant zero-order correlation between P3 amplitude and RT in response to canonical hands $(r[26]=-.48, p=.010)$, with higher P3 amplitude in response to canonical hands being related to faster math verification responses. Partial correlations showed that the correlation remained significant (nearing significance with corrected $\alpha$-level of .0125) after controlling for non-canonical P3 amplitude and RT to all six configurations $\left(r_{\text {partial }}[24]==.46, p=.018\right)$. On incorrect sum solution trials the zero-order correlation between $\mathrm{P} 3$ amplitude and reaction time in response to canonical finger patterns was not significant $(r[26]=-.30, p=.120)$ and became strongly non-significant after partialling out the effect of non-canonical P3 amplitude and RT $\left(r_{\text {partial }}[24]=-.03, p=.870\right)$. These correlation data suggest that there is a unique relation between $\mathrm{P} 3$ amplitude and reaction time only for canonical finger patterns and only when finger pattern and mentally computed sum were numerosity congruent (i.e., when finger montring patterns showed the correct sum solution). But based on the post-hoc and exploratory nature of these correlation analyses and the relatively small sample size these results should be interpreted with caution and need replication in a larger study sample. 
Table 4.1: Means and Standard Errors (between brackets) for behavioral and ERP data in the four CanonicityRange conditions separated for Solution Correctness and hemisphere.

\begin{tabular}{|c|c|c|c|c|c|c|}
\hline & & & \multicolumn{2}{|c|}{ Non-Canonical } & \multicolumn{2}{|l|}{ Canonical } \\
\hline \multirow{4}{*}{$\begin{array}{l}\text { Response Times } \\
\text { (milliseconds; ms) }\end{array}$} & Correct & Low Range & \multicolumn{2}{|c|}{$643.27(16.03)$} & \multicolumn{2}{|r|}{$585.53(16.03)$} \\
\hline & Solution & High Range & \multicolumn{2}{|c|}{$690.59(16.03)$} & \multicolumn{2}{|r|}{$644.58(16.07)$} \\
\hline & Incorrect & Low Range & \multicolumn{2}{|c|}{$663.13(14.89)$} & \multicolumn{2}{|r|}{$629.03(14.89)$} \\
\hline & Solution & High Range & \multicolumn{2}{|c|}{711.07 (14.89) } & \multicolumn{2}{|r|}{$716.82(14.89)$} \\
\hline \multirow{4}{*}{$\begin{array}{l}\text { Accuracy } \\
(\% \text { Correct })\end{array}$} & Correct & Low Range & \multicolumn{2}{|c|}{$78.47 \%(1.83 \%)$} & \multicolumn{2}{|r|}{$77.58 \%(1.33 \%)$} \\
\hline & Solution & High Range & \multicolumn{2}{|c|}{$71.41 \%(2.96 \%)$} & \multicolumn{2}{|r|}{$74.60 \%(2.28 \%)$} \\
\hline & Incorrect & Low Range & \multicolumn{2}{|c|}{$76.39 \%(2.01 \%)$} & \multicolumn{2}{|r|}{$75.69 \%(1.81 \%)$} \\
\hline & Solution & High Range & \multicolumn{2}{|c|}{$69.05 \%(2.88 \%)$} & \multicolumn{2}{|r|}{$70.04 \%(2.92 \%)$} \\
\hline \multirow{6}{*}{$\begin{array}{l}\text { P3 mV (Average } \\
\text { of CP and CPz at } \\
280-550 \mathrm{~ms} \text { ) }\end{array}$} & Correct & Low Range & & $3.66(0.32)$ & & $4.23(0.32)$ \\
\hline & Solution & High Range & & $2.94(0.32)$ & & $3.25(0.32)$ \\
\hline & Incorrect & Low Range & & $2.83(0.32)$ & & $3.44(0.32)$ \\
\hline & Solution & High Range & & $2.56(0.33)$ & & $2.91(0.32)$ \\
\hline & & & \multicolumn{2}{|c|}{ Non-Canonical } & \multicolumn{2}{|l|}{ Canonical } \\
\hline & & & Left & Right & Left & Right \\
\hline \multirow{4}{*}{$\begin{array}{l}\mathrm{P} 2 \mathrm{p} \mathrm{mV}(\mathrm{P} 3 \text { and } \mathrm{P} 4 \\
\text { at } 280-550 \mathrm{~ms})\end{array}$} & Correct & Low Range & $2.42(0.47)$ & $3.71(0.47)$ & $2.26(0.47)$ & $3.89(0.47)$ \\
\hline & Solution & & $1.99(0.47)$ & $2.56(0.47)$ & $1.95(0.47)$ & $2.26(0.47)$ \\
\hline & Incorrect & Low Range & $1.90(0.47)$ & $3.33(0.47)$ & $2.28(0.47)$ & $4.05(0.47)$ \\
\hline & Solution & High Range & $1.92(0.47)$ & $2.63(0.47)$ & $2.42(0.47)$ & $2.87(0.47)$ \\
\hline
\end{tabular}

Values shown are estimated marginal means with Standard Errors

Low Range $=$ Average of 2, 3 \& 4 I High Range $=$ Average of 7, 8 \& 9 


\subsection{Discussion}

The present study investigated two main hypotheses: 1) whether culture-specific (canonical) finger-number gestures (versus non-canonical gestures) facilitate magnitude comparison performance in a math verification task and whether this is related to facilitated semantic processing in the adult brain (the latter evidenced by higher amplitude of P2p and P3 amplitude components that have in earlier studies been linked to early and late stages of number semantic processing (see introduction) and 2) to investigate if canonical finger gestures showing small numbers 2-4 show stronger facilitation of math verification performance due to better semantic (memory) representation than canonical gestures showing larger numbers 7-9, as evidenced by faster comparison times and stronger $\mathrm{P} 2 \mathrm{p}$ and $\mathrm{P} 3$ responses.

The behavioral data showed a statistically significant interaction effect between numerical range, canonicity, and sum solution for reaction time. Further testing of this interaction showed that on trials in which finger patterns presented the correct sum solution (i.e., in which the magnitude of the computed sum and that indicated by the finger patterns was the same), both canonical small (2-4) and larger (7-9) finger patterns elicited faster magnitude comparison times than their non-canonical counterparts (i.e., a main canonicity effect was found for correct sum solution trials). These results replicate earlier reported facilitating effects of canonical finger-number configurations representing correct sum solutions on adults' reaction time in a math verification task (Badets et al., 2010). However, in this earlier study, canonical finger-counting patterns were not compared with noncanonical finger patterns but with quantity conveying non-finger stimuli (number of rods). Other prior studies that did compare effects of cardinal canonical and non-canonical finger patterns also reported similar facilitation effects of canonical fingers patterns 1-5 on reaction times, but in other number processing tasks (Di Luca et al., 2006; Di Luca et al., 2010; Di Luca and Pesenti, 2008; Soylu et al., 2019). Di Luca et al. (2006; 2010; 2008) concluded that these processing benefits of cardinal canonical finger configurations of numbers 1-5 are due to their storage in long-term memory, allowing for faster automatic recognition and retrieval of the numerosity they represent, whereas non-canonical finger patterns need elaboration (e.g., counting) to extract their numeric code. Our results add to these earlier behavioral findings, by making a distinction between canonical (and non-canonical) finger gestures showing small (2-4) or larger (7-9) quantities. Canonical finger-based representations of numbers 7-9 overall needed more processing time than those of numbers $2-4$, most likely caused by the need to add up the two numbers displayed by the two hands. Nevertheless, both canonical finger-based representations of numbers 
2-4 and 7-9 led to faster math verification responses on trials in which they presented the correct sum solution (i.e., in trials in which the to-be-compared stimuli were numerosity congruent).

The three-way interaction further showed that on trials in which finger patterns showed incorrect sum solutions (i.e., on numerosity incongruent trials), number-finger gestures 2-4 still facilitated comparison time compared to their non-canonical counterparts. However, the canonical finger patterns showing larger numbers 7-9 did no longer facilitate magnitude comparison (compared to non-canonical patterns 7-9) on trials where finger patterns showed an incorrect sum solution. A look at the mean reaction times shows that the abolishment of these processing benefits for canonical finger gestures 7-9 on incorrect sum solution trials is caused by a specific increase in reaction time for canonical patterns 7-9 on incorrect solution trials. Although speculative, this increase might be due to effects of numerical distance that are only present on incorrect sum solution trials where the mentally computed sum always differed with a numerical distance of 1 or 2 from the sum solution shown by the hands. A possible reason for distance effects only causing an increase in reaction time for incorrect canonical finger patterns 7-9 and not 2-4, might be that the latter have acquired stable, exact, one-to-one mapping onto spatial magnitude representations (i.e., the mental number line) causing them to be less susceptible to distortions caused by activation of adjacent numbers. In their priming study, Di Luca et al. (2010) confirmed exact mapping of canonical (and not non-canonical) finger patterns representing numbers 1-5 onto analog magnitude representations of number (e.g., number line). Such exact mapping was shown by the finding that canonical finger gesture primes showed strongest activation of target numbers to which they were close on the mental number line, with the extent of activation decreasing with an increase in the numeric distance between primes (the canonical finger patterns) and targets (Arabic digits or verbal numerals). Similar linear distance effects were not found for non-canonical finger patterns. Concluding, both small and larger canonical finger patterns benefitted magnitude comparison in the math verification task in numerosity congruent trials, but only canonical finger gestures 2-4 maintained the same processing benefits in more difficult numerosity incongruent trials where one had to make the decision that finger pattern and mentally computed sum did not show the same numerosity. As hypothesized in the introduction canonical finger patterns of small numbers 1-4 might have developed stronger (more stable) connections with other representations of magnitude due to the mediating/facilitating role that they play in early childhood when children learn to count and acquire the numeric meaning of the first four number symbols (Wynn, 1990, 1992). Recent models and findings suggest that especially 
the first four number symbols gain numeric meaning by mapping them with one-to-one correspondence on their earlier present, analog, non-symbolic representations of magnitude (Carey and Barner, 2019; Le Corre and Carey, 2007; Reynvoet and Sasanguie, 2016; van den Berg et al., 2020), and fingers may scaffold this difficult process of connecting abstract codes of number such as number words to their corresponding magnitude in the outside world Gunderson et al., 2015; Jay and Betenson, 2017.

The current study also measured ERPs, to gain more insight into the semantic processes underlying these facilitating effects of canonical finger-number representations on number symbol processing in the math verification task. In the early P2p time window from 220 to $310 \mathrm{~ms}$, a significant three-way interaction occurred between canonicity, numerical range, and hemisphere. A significantly enhanced positivity (P2p) was present above the right (but not left) parietal cortex in response to canonical (compared to non-canonical) finger-number montring configurations, but only for those in the low (2-4) numerical range, partially corroborating the stronger canonicity effect for finger patterns 2-4 than 7-9 on reaction time that was present for incorrect but not correct sum solution trials. P2p amplitude was only enhanced in response to canonical finger patterns 2-4 (and not patterns 7-9), and this $\mathrm{P} 2 \mathrm{p}$ response occurred independent of whether canonical finger patterns 2-4 matched or did not match with the mentally computed sum. As explained before, number comparison reaction time findings did however differ for correct and incorrect sum solution trials; on correct sum trials reaction time was faster for both canonical patterns 2-4 and 7-9. This can be explained by the fact that reaction times reflect the outcome of all previous information processing stages from early perception, early semantic processing to later more conscious memory and decision-making processes and these behavioral results are thus influenced by the (in)congruency of sum solutions. The P2p however represents a very early processing stage of automatic access to number semantics that is not affected by decision making processes, and such early numerical access is thus only shown for canonical patterns 2-4. The exploratory correlation analyses show no correlation between canonicity effects on $\mathrm{P} 2 \mathrm{p}$ amplitude and reaction time, whereas this is the case for the later P3, leading to the tentative conclusion that the facilitating effects of canonical finger patterns on RT are likely due to facilitated processing during later semantic processing stages (see below for further discussion). No significant canonicity effects on the left- or right parietal P2p component were present for finger montring postures showing numbers 7-9. As discussed in the introduction, a previous MEG study by Nakamura et al. (Nakamura et al., 2004) found similarly enhanced activation in posterior areas to meaningful versus meaningless (non-numeric) 
hand gestures within approximately the same time-interval and with a similar right-hemisphere dominance as the current P2p effect. The authors concluded that whereas the left hemisphere is more involved in early visual-motor processing of the hand stimuli, the right hemisphere is involved in semantic processing of the cultural meaningful hand gestures. Accordingly, the current higher right-lateralized $\mathrm{P} 2 \mathrm{p}$ response to canonical finger-number patterns 2-4 might be a sign of the automatic activation of number semantics, more specifically the activation of associated magnitude representations in long-term memory.

Whereas not including finger stimuli, several prior ERP, fMRI, and behavioral studies provide support for a conclusion of the current right-lateralized $\mathrm{P} 2 \mathrm{p}$ effect reflecting activation of magnitude representations associated with canonical finger patterns of numbers smaller than five. First, as reviewed in the introduction, multiple prior number processing ERP studies provide evidence for a link between the P2p and access to (spatial) representations of magnitude (e.g., on a mental number line). In a study by Dehaene et al. (1996), adult participants performed a symbolic number comparison task requiring magnitude comparisons between digits 1-9, which requires activation/retrieval of a digit's magnitude code. A right-lateralized ERP component, similar to the present P2p in timing and location, called the P2p, was modulated by the numerical distance between the to-be-compared numbers, and was hence concluded to represent access to magnitude information. Further, in a recent ERP study by van den Berg et al. (2020) young adults learned the numerical meaning of novel symbols representing numbers 1-9 by mapping them onto corresponding quantity representations, mimicking the symbolic non-symbolic mapping process in children. A P2p-ERP component with similar right-parietal lateralization and timing as the current $\mathrm{P} 2 \mathrm{p}$ gradually increased in amplitude as the novel symbols acquired numeric meaning by their mapping onto their non-symbolic counterparts. This effect did however only occur for novel symbols representing small magnitudes 1-4 that could be easily mapped onto corresponding non-symbolic representations via subitizing. In line with Dehaene et al. (1996), this led to the conclusion that the $\mathrm{P} 2 \mathrm{p}$ reflects the (automatic) activation of magnitude information that has become associated with the novel symbols during learning. Second, multiple fMRI studies have found specific activation of right inferior parietal sulcus during number comparison tasks in which adults had to decide which of two symbolic (e.g., Arabic digits) or non-symbolic (arrays of dots) number stimuli represented a larger magnitude and concluded that this area houses an analog representation of magnitude and is involved in automatic or voluntary semantic processing of the magnitude dimension (Chochon et al., 1999; Eger 
et al., 2003; Holloway et al., 2010; Pinel et al., 2001). Whereas previous fMRI studies included standard symbolic or non-symbolic stimuli (Arabic digits or dot patterns), a link between right parietal activation and magnitude processing is also reported for finger-number stimuli in an fMRI study by Kaufmann et al. (2008). In this study, children and adults showed unique activation of right parietal areas during performance of a task in which they had to indicate which of two adjacently presented one-hand finger patterns showed the largest magnitude and not in a task with the same hand stimuli but now requiring the comparison of hand palm orientations (spatial comparison). Third and last, there are prior reports of canonical (cultural-specific) counting habits such as starting to count from 1-10 with the left or right hand, creating biases in adults' performance on tasks that require processing of spatial-numerical relations, like positioning a number on a number line (Fabbri and Natale, 2016; Fischer, 2008).

Based on the findings from the prior brain studies discussed in the previous paragraph, we tentatively suggest that the current higher right-parietal P2p response to canonical finger montring postures showing numbers 2-4 reflects first activation of/or access to associated analog magnitude representations. One explanation for this $\mathrm{P} 2 \mathrm{p}$ effect being only present for small one-hand finger patterns (2-4) and not for finger patterns 7-9, might be that fingermagnitude associations are established in the brain during the developmental pre-school phase in which children first use their fingers to acquire numerical representations (Fischer, 2008), which primarily involves small numbers $1-4$. The semantic associations between canonical finger montring postures representing numbers 1-5 and their corresponding non-symbolic (magnitude) representation might be further strengthened by their more frequent use in daily communication. It is currently not clear why the same $\mathrm{P} 2 \mathrm{p}$ response is not elicited by canonical patterns showing numbers large than 5, but this might be due to weaker direct associations with non-symbolic number representations, although this is speculative at this stage. It should be noted that small (2-4) and larger (7-9) canonical finger montring patterns facilitated math verification performance and enhanced P3 amplitudes to the same extent, the latter pointing to a special status of both in long-term memory. Although purely speculative, it might be that two-hand finger montring patterns that are not yet as frequently used in early childhood, have acquired stronger associations with other, later acquired symbolic representations of number than with non-symbolic representations. Last, although the above functional interpretation of the current P2p findings for canonical one-hand patterns fits with our and the reviewed data, the current study can however not confirm whether the current right lateralized parietal P2p data can be explained by a source in right parietal cortex and this thus needs confirmation in future studies. 
In contrast to the $\mathrm{P} 2 \mathrm{p}$ that was only enhanced in response to canonical finger patterns showing numbers smaller than 5, the later centro-parietal P3-ERP component did not show the predicted canonicity $\mathrm{x}$ numerical range interaction but instead showed a main effect of canonicity, with $\mathrm{P} 3$ amplitude being enhanced to both canonical montring patterns 2-4 and 7-9. The present main canonicity effect on the P3 resembles that reported by Soylu et al. (2019) in timing and location. These authors compared ERP responses elicited by canonical (montring and counting) and non-canonical finger-number stimuli showing numbers 1-4 and interpreted the enhanced $\mathrm{P} 3$ response to canonical finger patterns 1-4 as reflecting the retrieval of semantic, in this case numeric, information associated with the canonical finger-number patterns from memory. We extended these findings by also showing a P3 amplitude canonicity effect for canonical finger montring patterns 7-9 represented on two hands. We furthermore found a unique significant correlation between P3 amplitude and reaction time for canonical finger patterns showing correct sum solutions, with higher P3 amplitude being associated with faster sum verification times. But regarding the post-hoc and exploratory nature of the correlation analyses and the relatively small sample size of 28 adults, these correlation results should be carefully interpreted and need replication within a larger sample. Comparison of P3 results between Soylu's et al. (2019) study and the present study is however complicated by differences in the tasks used and the moments in the task during which ERPs to the finger patterns were measured. Whereas in Soylu et al. (2019) ERPs were measured in response to finger patterns that preceded Arabic digits to which their numerosity had to be matched, in our task ERPs were measured in response to the finger patterns showing the solution to an earlier presented addition problem. In the present study, ERPs do thus not only reflect processes associated with the identification/extraction of the numerosity shown by the finger montring patterns but also reflect the processes involved in making the decision of whether the number indicated by the finger patterns matches with the solution of the prior presented addition problem or not (i.e., number comparison processes). In prior ERP studies using similar math verification paradigms but with sum solutions shown in the form of Arabic digits instead of finger montring patterns, similarly enlarged P3 amplitude on correct (versus incorrect) sum solutions trials has been consistently found and is linked to easier classification/categorization processes (e.g., Dickson and Wicha, 2019; Polich, 1987), and/or higher intuitive level of response confidence (Eimer and Mazza, 2005; Ye et al., 2019). Dickson and Wicha (2019) also found an effect of problem size with smaller math problems eliciting higher $\mathrm{P} 3$ responses, which they explained by smaller problems having gained stronger internalized representations and thus higher familiarity due to 
our more frequent encounters with small math problems in daily life. Interestingly, in addition to the main P3 canonicity effect on P3 amplitude, we also found an interaction between numerical range and sum solution. This interaction indicated that finger patterns conveying small numerosities 2-4 showed a larger correct/incorrect P3 increase than finger patterns 7-9. In line with the previous P3- and math verification ERP literature, we tentatively interpret the current P3 findings and their correlation with math verification time as signifying facilitated response decision/categorization processes of more familiar and easily recognizable canonical one-hand finger montring patterns, probably due to their stronger internalized representations in long-term memory.

In summary, cardinal canonical finger patterns of both numbers 2-4 and 7-9 facilitated young adults' sum verification time when showing correct sum solutions. This behavioral facilitation was accompanied by an enhanced P3 response, which, based on prior literature, might be indicative of easier recognition/classification of cardinal canonical finger montring patterns due to their storage in long-term memory. Canonical finger montring patterns showing small numbers 2-4 in addition also facilitated performance on incorrect sum solution trials and elicited an early $\mathrm{P} 2 \mathrm{p}$ response above right parietal cortex that was, based on prior brain studies, suggested to reflect the (automatic) activation of/access to associated analog representations of magnitude possibly originating from their frequent use in early childhood. Future longitudinal studies measuring children through different developmental phases should shed more light on when and how these cardinal finger patterns gain their canonical status, and how beneficial they are in the developmental phase in which children acquire the -symbolic number system.

\section{Data Availability}

The data files containing the raw behavioral data and the SPSS matrix containing the behavioral and ERP data used in the presented analyses will be available via the DataverseNL repository, https://doi.org/10.34894/4OEBD9. The raw EEG-data will be available upon reasonable request by sending an e-mail to 1.jonkman@maastrichtuniversity.nl

\section{Author contributions statement}

FvdB and LMJ conceived of and designed the experiment. FvdB collected and processed the data. FvdB and LMJ interpreted the results and wrote the manuscript. PdW provided critical content and helped revising the manuscript. All authors read and approved the final manuscript. 


\subsection{References}

Analytics, I. (2021). Ibm spss I ibm analytics. (Cited on page 147).

Avikainen, S., Liuhanen, S., Schürmann, M., \& Hari, R. (2003). Enhanced extrastriate activation during observation of distorted finger postures. Journal of Cognitive Neuroscience, 15(5), 658-663. https://doi.org/10.1162/jocn.2003.15.5.658 (cited on pages 139,147$)$

Badets, A., Pesenti, M., \& Olivier, E. (2010). Response-effect compatibility of fingernumeral configurations in arithmetical context. Quarterly Journal of Experimental Psychology, 63(1), 16-22. https://doi.org/10.1080/17470210903134385 (cited on pages $137,138,140,141,156)$

Brainvision analyzer (vers. 2.2.0)[software]. (2019). (Cited on page 146).

Butterworth, B. (1999). The mathematical brain (illustrate). Macmillan. (Cited on page 136).

Carey, S. (2009). The origin of concepts (Volume 133). Oxford University Press. https: //doi.org/10.1093/acprof:oso/9780195367638.001.0001. (Cited on page 137)

Carey, S., \& Barner, D. (2019). Ontogenetic origins of human integer representations. Trends in Cognitive Sciences, 23(10), 823-835. https://doi.org/10.1016/j.tics. 2019.07.004 (cited on pages 136, 137, 141, 158)

Chochon, F., Cohen, L., van de Moortele, P. F., \& Dehaene, S. (1999). Differential contributions of the left and right inferior parietal lobules to number processing. Journal of cognitive neuroscience, 11(6), 617-30. https://doi.org/10.1162/089892999563689 (cited on page 159)

Dehaene, S. (1996). The organization of brain activations in number comparison: Event-related potentials and the additive-factors method. Journal of Cognitive Neuroscience, 8(1), 47-68. https://doi.org/10.1162/jocn.1996.8.1.47 (cited on pages $138,139,141,147,159)$

Della Sala, S., \& Anderson, M. (2012). Neuroscience in education: The good, the bad, and the ugly. Oxford University Press. https://doi.org/10.1093/acprof:oso/ 9780199600496.001.0001. (Cited on page 138)

Delorme, A., \& Makeig, S. (2004). Eeglab: An open source toolbox for analysis of single-trial eeg dynamics including independent component analysis. Journal of Neuroscience Methods, 134(1), 9-21. https://doi.org/https://doi.org/10.1016/j. jneumeth.2003.10.009 (cited on page 146)

Di Luca, S., Granà, A., Pesenti, M., Semenza, C., \& Seron, X. (2006). Finger-digit compatibility in arabic numeral processing. Quarterly Journal of Experimental 
Psychology, 59(9), 1648-1663. https://doi.org/10.1080/17470210500256839 (cited on pages $137,138,156)$

Di Luca, S., Lefèvre, N., \& Pesenti, M. (2010). Place and summation coding for canonical and non-canonical finger numeral representations. Cognition, 117(1), 95-100. https://doi.org/10.1016/j.cognition.2010.06.008 (cited on pages 137, 138, 140, $141,156,157)$

Di Luca, S., \& Pesenti, M. (2008). Masked priming effect with canonical finger numeral configurations. Experimental Brain Research, 185(1), 27-39. https://doi.org/10. 1007/s00221-007-1132-8 (cited on pages 137, 138, 156)

Dickson, D. S., \& Wicha, N. Y. (2019). P300 amplitude and latency reflect arithmetic skill: An erp study of the problem size effect. Biological Psychology, 148, 107745. https://doi.org/10.1016/j.biopsycho.2019.107745 (cited on pages 141, 142, 161)

Domahs, F., Krinzinger, H., \& Willmes, K. (2008). Mind the gap between both hands: Evidence for internal finger-based number representations in children's mental calculation. Cortex, 44(4), 359-367. https://doi.org/10.1016/j.cortex.2007.08.001 (cited on page 136)

Eger, E., Sterzer, P., Russ, M. O., Giraud, A. L., \& Kleinschmidt, A. (2003). A supramodal number representation in human intraparietal cortex. Neuron, 37(4), 719-725. https://doi.org/10.1016/S0896-6273(03)00036-9 (cited on page 159)

Eimer, M., \& Mazza, V. (2005). Electrophysiological correlates of change detection. Psychophysiology, 42(3), 328-342. https://doi.org/10.1111/j.1469-8986.2005. 00285.x (cited on page 161)

Fabbri, M., \& Natale, V. (2016). Finger counting and (2d:4d) digit ratio in spatial-numerical association. Perception, 45(1-2), 136-155. https://doi.org/10.1177/0301006615594913 (cited on page 160)

Fischer, M. H. (2008). Finger counting habits modulate spatial-numerical associations. Cortex, 44(4), 386-392. https://doi.org/10.1016/j.cortex.2007.08.004 (cited on page 160)

Fuson, K. C. (1988). Children's counting and concepts of number (Illustrate, Volume 31). Springer New York. https://doi.org/10.1007/978-1-4612-3754-9. (Cited on page 136)

Geary, D. C. (2004). Mathematics and learning disabilities. Journal of Learning Disabilities, 37(1), 4-15. https://doi.org/10.1177/00222194040370010201 (cited on page 136) 
Gelman, R., \& Gallistel, C. R. (1978). The child's understanding of number. Harvard University Press. (Cited on page 136).

Gracia-Bafalluy, M., \& Noël, M. P. (2008). Does finger training increase young children's numerical performance? Cortex, 44(4), 368-375. https://doi.org/10.1016/j.cortex. 2007.08.020 (cited on page 138)

Gunderson, E. A., Spaepen, E., Gibson, D., Goldin-Meadow, S., \& Levine, S. C. (2015). Gesture as a window onto children's number knowledge. Cognition, 144, 14-28. https://doi.org/10.1016/j.cognition.2015.07.008 (cited on pages 137, 158)

Holloway, I. D., Price, G. R., \& Ansari, D. (2010). Common and segregated neural pathways for the processing of symbolic and nonsymbolic numerical magnitude: An fmri study. NeuroImage, 49(1), 1006-1017. https://doi.org/10.1016/j. neuroimage.2009.07.071 (cited on page 160)

Jay, T., \& Betenson, J. (2017). Mathematics at your fingertips: Testing a finger training intervention to improve quantitative skills. Frontiers in Education, 2, 22. https: //doi.org/10.3389/feduc.2017.00022 (cited on pages 137, 158)

Jiang, T., Qiao, S., Li, J., Cao, Z., Gao, X., Song, Y., Xue, G., Dong, Q., \& Chen, C. (2010). Effects of symbol type and numerical distance on the human eventrelated potential. Neuropsychologia, 48(1), 201-210. https://doi.org/10.1016/j. neuropsychologia.2009.09.005 (cited on pages 139, 147)

Kaufmann, L., Vogel, S. E., Wood, G., Kremser, C., Schocke, M., Zimmerhackl, L. B., \& Koten, J. W. (2008). A developmental fmri study of nonsymbolic numerical and spatial processing. Cortex, 44(4), 376-385. https://doi.org/10.1016/j.cortex.2007. 08.003 (cited on page 160)

Keil, A., Debener, S., Gratton, G., Junghöfer, M., Kappenman, E. S., Luck, S. J., Luu, P., Miller, G. A., \& Yee, C. M. (2014). Committee report: Publication guidelines and recommendations for studies using electroencephalography and magnetoencephalography. Psychophysiology, 51(1), 1-21. https://doi.org/10.1111/psyp.12147 (cited on pages 151, 152)

Klein, E., Moeller, K., Willmes, K., Nuerk, H. C., \& Domahs, F. (2011). The influence of implicit hand-based representations on mental arithmetic. Frontiers in Psychology, 2(SEP), 197. https://doi.org/10.3389/fpsyg.2011.00197 (cited on page 136)

Le Corre, M., \& Carey, S. (2007). One, two, three, four, nothing more: An investigation of the conceptual sources of the verbal counting principles. Cognition, 105(2), 395-438. https://doi.org/10.1016/j.cognition.2006.10.005 (cited on pages 136, 141, 158) 
Libertus, M. E., Woldorff, M. G., \& Brannon, E. M. (2007). Electrophysiological evidence for notation independence in numerical processing. (Volume 3). https://doi.org/ 10.1186/1744-9081-3-1. (Cited on pages 138, 139, 141, 147)

Lopez-Calderon, J., \& Luck, S. J. (2014). Erplab: An open-source toolbox for the analysis of event-related potentials. Frontiers in Human Neuroscience, 8(1 APR), 213. https://doi.org/10.3389/fnhum.2014.00213 (cited on pages 146, 151, 152)

MATLAB. (2019). 9.6 (r2019b). The MathWorks Inc. (Cited on page 146).

Moeller, K., Martignon, L., Wessolowski, S., Engel, J., \& Nuerk, H. C. (2011). Effects of finger counting on numerical development the opposing views of neurocognition and mathematics education. Frontiers in Psychology, 2(NOV), 328. https://doi. org/10.3389/fpsyg.2011.00328 (cited on page 136)

Nakamura, A., Maess, B., Knösche, T. R., Gunter, T. C., Bach, P., \& Friederici, A. D. (2004). Cooperation of different neuronal systems during hand sign recognition. NeuroImage, 23(1), 25-34. https://doi.org/10.1016/j.neuroimage.2004.04.034 (cited on pages $139,147,158$ )

Noël, M. P., Gracia-Bafalluy, M., \& Noël, M. P. (2005). Finger gnosia: A predictor of numerical abilities in children? Cortex, 11(5), 413-430. https://doi.org/10.1080/ 09297040590951550 (cited on page 138)

Paulsen, D. J., \& Neville, H. J. (2008). The processing of non-symbolic numerical magnitudes as indexed by erps. Neuropsychologia, 46(10), 2532-2544. https://doi.org/10.1016/j.neuropsychologia.2008.04.003 (cited on pages 139, 147)

Peirce, J., Gray, J. R., Simpson, S., MacAskill, M., Höchenberger, R., Sogo, H., Kastman, E., \& Lindeløv, J. K. (2019). Psychopy2: Experiments in behavior made easy. Behavior Research Methods, 51(1), 195-203. https://doi.org/10.3758/s13428018-01193-y (cited on page 144)

Petrilla, A. (2012, August 8). Binary counting on fingers [[Online; accessed 2021-02-26]]. http://apetrilla.blogspot.com/2012/08/binary-counting-on-fingers.html. (Cited on page 145)

Pinel, P., Dehaene, S., Rivière, D., \& LeBihan, D. (2001). Modulation of parietal activation by semantic distance in a number comparison task. NeuroImage, 14(5), 1013-1026. https://doi.org/10.1006/nimg.2001.0913 (cited on pages 138, 139, $147,160)$

Polich, J. (1987). Task difficulty, probability, and inter-stimulus interval as determinants of p300 from auditory stimuli. Electroencephalography and Clinical 
Neurophysiology/ Evoked Potentials, 68(4), 311-320. https://doi.org/10.1016/0168-5597(87)90052-9 (cited on page 161)

Polich, J. (2007). Updating p300: An integrative theory of p3a and p3b. Clinical Neurophysiology, 118(10), 2128-2148. https://doi.org/10.1016/j.clinph.2007.04.019 (cited on pages 139, 147)

Proverbio, A. M., \& Carminati, M. (2019). Finger-counting observation interferes with number processing. Neuropsychologia, 131, 275-284. https://doi.org/10.1016/j. neuropsychologia.2019.06.001 (cited on pages 140-142)

Reynvoet, B., \& Sasanguie, D. (2016). The symbol grounding problem revisited: A thorough evaluation of the ans mapping account and the proposal of an alternative account based on symbol-symbol associations. Frontiers in Psychology, 7(OCT), 1581. https://doi.org/10.3389/fpsyg.2016.01581 (cited on pages $136,141,158)$

Soylu, F., Lester Jr, F. K., Newman, S. D., Norton, A., \& Nurnberger-Haag, J. (2018). You can count on your fingers: The role of fingers in early mathematical development. Journal of Numerical Cognition, 4(1), 2363-8761. https://doi.org/10.5964/jnc. v4i1.85 (cited on pages 136, 138)

Soylu, F., Rivera, B., Anchan, M., \& Shannon, N. (2019). Erp differences in processing canonical and noncanonical finger-numeral configurations. Neuroscience Letters, 705, 74-79. https://doi.org/10.1016/j.neulet.2019.04.032 (cited on pages 138, $140,141,147,156,161)$

Turconi, E., Jemel, B., Rossion, B., \& Seron, X. (2004). Electrophysiological evidence for differential processing of numerical quantity and order in humans. Cognitive Brain Research, 21(1), 22-38. https://doi.org/10.1016/j.cogbrainres.2004.05.003 (cited on pages 138, 139, 141, 147)

van den Berg, F. C., de Weerd, P., \& Jonkman, L. M. (2020). Number-related brain potentials are differentially affected by mapping novel symbols on small versus large quantities in a number learning task. Journal of cognitive neuroscience, 32(7), 1263-1275. https://doi.org/10.1162/jocn_a_01546 (cited on pages 136, $139,141,147,158,159)$

Wynn, K. (1990). Children's understanding of counting. Cognition, 36(2), 155-193. https: //doi.org/10.1016/0010-0277(90)90003-3 (cited on pages 136, 157)

Wynn, K. (1992). Children's acquisition of the number words and the counting system. Cognitive Psychology, 24(2), 220-251. https://doi.org/10.1016/0010-0285(92) 90008-P (cited on pages 136,157 ) 
Ye, M., Lyu, Y., Sclodnick, B., \& Sun, H. J. (2019). The p3 reflects awareness and can be modulated by confidence. Frontiers in Neuroscience, 13(MAY), 510. https: //doi.org/10.3389/fnins.2019.00510 (cited on page 161) 


\section{CANONICAL FINGER-NUMBER

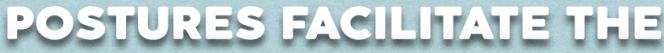

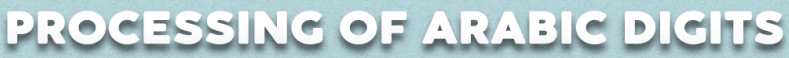 IN ADULUS: AN EVENU-R POUENULAL STUDY}

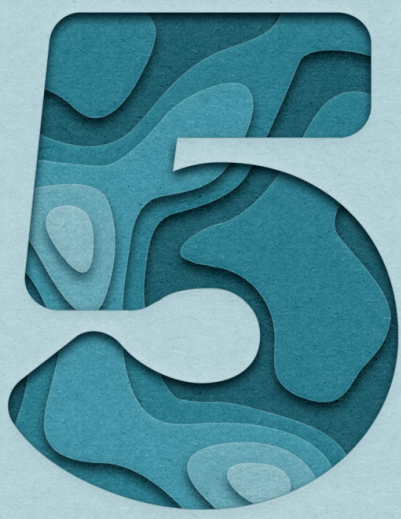

\subsection{Abstract}

Various studies claim that early-learned, culturally determined (canonical) finger-number postures have stronger connections to numerical concepts stored in long-term memory than unfamiliar finger postures, allowing for faster access to their numerical meaning. The current study investigated whether canonical finger-number postures gesturing numbers 1-4 or 6-9, facilitate the behavioral and neural processing of Arabic numerals in adults in a number comparison task. Thirty-one adults had to decide whether simultaneously presented Arabic numerals and canonical or noncanonical finger-number postures showed the same or a different numerosity while measuring performance and Event-Related Potentials (ERPs). The results showed faster responses when comparisons involved canonical (versus non-canonical) finger-number postures, but only on numerosity-congruent trials where finger posture and Arabic numeral matched in number identity. Canonical, and small-number finger-patterns 1-4 in general (irrespective of their canonicity), also elicited enhanced early right-parietal $\mathrm{P} 2 \mathrm{p}$ and later centro-parietal P3-ERP responses on numerosity-congruent trials. We suggest these P2p and P3 findings respectively reflect facilitated numerical access and easier categorization of canonical finger-number postures. The current results provide behavioral and neurophysiological evidence for the embodiment of culture-specific, canonical, finger-number postures and their link with other number symbol representations in the adult brain, likely emerging from their more frequent use in daily life communication and/or in early childhood during number symbol acquisition.

R Corresponding reference: van den Berg, F. C. G., De Weerd, P., \& Jonkman, L. M. (2021, under review) Canonical finger-number postures facilitate the processing of Arabic digits in adults: an Event-Related Potential study. Neuropsychologia 


\subsection{Introduction}

Humans possess the unique ability to represent quantities abstractly in the form of number words or symbols (e.g., Arabic numerals like 3 or 6 ). How young children learn to associate these abstract words/symbols with the quantities that they represent is still largely unknown, but it has been shown to be a lengthy and complex process (Wynn, 1992). There is evidence that embodied learning, that is learning involving the use of (parts of) the body, can make it easier to attach semantic meaning to symbols and abstract concepts (Lindemann and Fischer, 2015; Tran et al., 2017). With regard to numeracy development, an example of embodied learning is children's intuitive use of fingers when learning to count or when performing simple addition or subtraction (Butterworth, 1999; Fuson, 1988; Moeller et al., 2012; Siegler and Shrager, 1984). In the case of number word or symbol learning early in development, fingers might play a special facilitating role by providing concrete (and always available) referents of quantity, thereby facilitating the process of associating abstract number words or symbols with the quantity that they represent (Butterworth, 1999; U. Fischer et al., 2011; Fuson, 1988; Knudsen et al., 2014).

Whereas some suggest that fingers only act as temporary helpful aids during early number symbol acquisition (see Moeller et al., 2011 for a review), there is increasing evidence from behavioral studies that internalized (embodied) finger-number representations still influence or facilitate number symbol processing in adults (for a review see Di Luca and Mauro, 2011). This points to the existence of interlinked fingerand other symbolic or non-symbolic number representations. The current study aimed to add to the understanding of the processing and internalization (embodiment) of canonical finger-number postures and their potential facilitating effects on the processing of other number symbols (e.g. , Arabic digits) in adults, by measuring Event Related brain Potentials (ERPs). To that aim, we investigated whether early-learned, culture-specific (also called canonical) finger-number postures (versus unfamiliar postures) facilitate the processing of Arabic digits in a same-different number comparison task and whether this is accompanied by electrophysiological evidence of facilitated semantic processing in the brain, which would be evidence for their embodiment.

As mentioned above, several behavioral studies using different paradigms have already provided behavioral evidence for canonical finger-number postures facilitating performance in numerical processing tasks in adults (Badets et al., 2010; Di Luca et al., 2006; Di Luca and Pesenti, 2008). Two different types of canonical finger-number postures have been used in these studies, referred to as 'counting' or 'montring' postures (Di Luca and 
Pesenti, 2008). Both counting and montring postures are culturally determined, with different cultures showing distinct patterns (Butterworth, 1999; Maor and Ifrah, 2001). In western cultures counting and montring postures consist of a canonical pattern of simultaneously raised fingers communicating a number in the 1-10 range, but 'counting' postures involve the thumb and montring postures do not (at least for numbers 1-4). Di Luca \& Pesenti (2008) did not find any differences in number identification speed between canonical counting and montring finger postures showing numbers 1-5, and both types of canonical postures have been reported to have facilitating effects on other symbolic number processing tasks or skills in adults or children (Badets et al., 2010; Di Luca et al., 2010; Di Luca and Pesenti, 2008; Lafay et al., 2013; but see Soylu et al., 2019, who reported behavioral facilitation of only montring and not counting postures 1-5). In the following text the term canonical finger-number postures is used to refer to culturally familiar counting or montring finger-based number postures used for number communication.

Several authors have suggested that the facilitated processing of canonical fingernumber postures are caused by canonical patterns becoming embodied and developing links with other numerical representations due to their frequent encounter and use in daily life (Di Luca and Mauro, 2011; M. H. Fischer, 2012). Di Luca and colleagues (2008) presented the first evidence that adults were faster in retrieving the number words for canonical finger-number montring postures than for unfamiliar, non-canonical, finger postures (e.g., thumb, index finger and pinky showing number three). In the same paper, the authors also demonstrated that being primed by canonical (as opposed to non-canonical) finger-montring postures led to faster naming of subsequently presented Arabic numerals. In a follow-up study, Di Luca et al. (Di Luca et al., 2010) further demonstrated that the larger the numerical distance was between the numerosities represented by the canonical finger-number prime and the target (the Arabic numeral), the smaller the priming benefits were. The authors interpreted this effect as canonical finger representations of numbers 1-5 having a direct link with numbers represented on the number line (Di Luca et al., 2010). This can be conceptualized as the perception of a canonical finger number pattern showing two (index and middle finger raised), most strongly activating the number two on the number line, with activation decreasing the further numbers are away from the number two on the number line. Sixtus et al. (2017) demonstrated similar priming effects when participants produced the finger-number gestures themselves (instead of viewing them in a picture as in Di Luca et al., 2010). Finally, Badets et al. (2010) reported facilitating effects of canonical finger-number counting postures on number symbol processing in a math verification task. Adults were faster at deciding whether a sum solution was 
correct when the solution was shown by a canonical finger-number pattern instead of in the form of several rods. In a recent study we replicated this positive effect of canonical finger-number postures on math verification time (van den Berg et al., 2021), but now comparing canonical postures with non-canonical postures instead of with rods as in the Badets et al. (2010) study. The behavioral results from above discussed studies suggest that canonical finger-number postures (whether counting or montring) have a special status as learned patterns stored in long-term memory that are quickly accessible and capable of automatically activating other numerical representations, thereby possibly facilitating their processing (Di Luca and Mauro, 2011).

Brain studies could provide more direct evidence of embodiment of canonical fingernumber representations and their link to other representations of number. However, there are to the best of our knowledge only three prior studies that have used Eventrelated brain potentials (ERPs) to investigate the cerebral processing of finger-number postures/representations. In a study by Proverbio and Carminati (2019), adults performed a math verification task in which participants determined the correctness of the solution to a math problem presented in Arabic numerals in the 0-10 range. Task-irrelevant fingercounting patterns flanked the solution (Arabic digit), showing either a congruent (same as the Arabic numeral) or incongruent quantity (different from the numeral). In contrast to the above discussed behavioral studies that all reported facilitating effects of perception or production of canonical finger-number patterns on number symbol processing, Proverbio and Carminati (2019) reported a decrease in accuracy when congruent finger-counting patterns flanked sum solutions, indicative of interference. The behavioral interference was accompanied by an enhanced positivity above the fronto-central cortex between 700-900 milliseconds after stimulus presentation in the ERPs. These contrasting results are likely explained by differences in task design; in the Proverbio and Carminati (2019) study, fingercounting patterns were irrelevant to the task and did not require active processing. All above discussed studies that did find facilitated processing of canonical finger-number postures used task paradigms that demanded active retrieval of the numeric value presented by the finger-number postures. Additionally, the conclusion that only early-learned, canonical finger-number representations have acquired a special status in long-term memory requires a design that includes a direct comparison between the brain processing of canonical and non-canonical finger-number configurations which was not the case in the above study. 
Soylu et al. (2019) did compare ERP responses to canonical and non-canonical finger-number stimuli representing numbers 1-4. In their study, participants determined whether finger counting patterns, canonical finger montring patterns, and non-canonical finger configurations showed the same or a different number than a subsequently presented Arabic numeral. Replicating results from earlier behavioral studies by Di Luca et al. (2006; 2008), canonical montring patterns of numbers 1-4 led to faster and more accurate responses than counting- or non-canonical patterns which was accompanied by enhanced amplitude of early exogenous P1 and N1 ERP components indexing higher automatic attraction of attention of the montring patterns. The later P3-activity measured in a 250-500 ms post-stimulus time window at central and lateral centro-parietal/parietal and occipital electrodes showed enhanced amplitude to both counting and montring patterns, which was, based on the prior P3 memory literature (Donchin, 1981; Soylu et al., 2019), interpreted as indexing the retrieval of the numeric value represented by the familiar finger posture from memory. The posterior location and timing of this finger-number canonicity effect on the ERP overlaps with that reported in earlier magnetoencephalography (MEG) studies investigating the semantic processing of other (non-numeric) culturally meaningful hand gestures (Avikainen et al., 2003; Nakamura et al., 2004). These combined findings thus suggest that posterior activity in this mid-latency time window indexes the processing stage in which one has first access to, or extracts the semantic meaning of earlier learned, culturally meaningful hand gestures, including quantity gestures.

The studies discussed above either studied the processing of finger-number patterns representing small numerosities 1-4 (Di Luca et al., 2010; Soylu et al., 2019) or did not distinguish between single- and double-handed gestures of respectively numbers 1-5 and 6-10 in their analyses (Di Luca and Pesenti, 2008; Proverbio and Carminati, 2019). Since there might be differences in the frequency of use and thus the embodiment of canonical finger-number postures for quantities 1-4 represented on a single hand and higher quantities represented on both hands, they might also have different facilitating effects on number symbol processing. In a recent study we did investigate such processing differences between canonical and non-canonical postures showing one-hand numbers 2-4 and two-hand numbers 7-9 on reaction time and P3 amplitude (and also included the not earlier studied P2p-ERP component) using a math verification paradigm (van den Berg et al., 2021). In this study, adult participants mentally computed the solution to a simple addition problem and had to compare their solution kept in memory with the presented sum solution which could be the correct or incorrect solution. We found that compared to non-canonical postures, both one-hand and two-hand canonical finger postures facilitated 
(sped up) the number comparison process to the same extent and this went along with an enhanced parietal P3 response indicative of facilitated categorization/memory retrieval for both small and larger canonical finger postures. A response of the earlier P2p-ERP component was however only elicited by one-hand canonical finger postures (compared to non-canonical postures) showing numbers 2-4. Based on the prior number processing-ERP literature (Dehaene, 1996; Hyde and Spelke, 2012; Libertus et al., 2007; Temple and Posner, 1998, this enhanced early P2p response to only canonical finger postures 2-4 was suggested to reflect the automatic activation of non-symbolic (analog) representations of number that became associated with these patterns due to their frequent use early in development and life.

The aim of the present study was to see if we could replicate these P2p and P3 results in a Stroop-like paradigm in which finger-number postures and Arabic digits 1-4 and 6-9 were presented within one stimulus display (instead of sequentially as in Soylu et al., 2019 and in van den Berg et al., 2021). Proverbio \& Carminati (2019) also presented Arabic digits and finger-number stimuli within one stimulus display, but in a math verification task in which finger-stimuli were task-irrelevant and also no non-canonical postures were shown. In the present study participants had to actively compare simultaneously presented (canonical/non-canonical) finger postures and Arabic digits (in numerical ranges 1-4 and 6-9) in order to decide whether finger-postures and Arabic digit showed the same or a different numerosity. The task used in the current study is more comparable to number comparison tasks often used in the number processing literature in which one has to decide whether different standard symbolic (words/digits) or non-symbolic (e.g., arrays of dots) stimuli represent the same or a different magnitude. In line with above reviewed finger-pattern processing studies (Badets et al., 2010; Di Luca et al., 2006; Di Luca et al., 2010; Di Luca and Pesenti, 2008; Sixtus et al., 2017; Soylu et al., 2019; van den Berg et al., 2021), we expected canonical finger-number postures to elicit faster number comparison responses than non-canonical postures, especially in numerosity congruent trials where Arabic numerals and finger-number postures indicate the same numerosity (Badets et al., 2010; Sixtus et al., 2017; van den Berg et al., 2021). Additionally, in line with the above discussed finger-number ERP studies and the number comparison-ERP literature, we expected two specific ERP components to be modulated by the canonicity of the finger postures. The first being the $\mathrm{P} 2 \mathrm{p}$, in the number processing ERP literature associated with the automatic activation of the semantic (magnitude) information associated with number symbols (Dehaene, 1996; Libertus et al., 2007; Temple and Posner, 1998). Based on our earlier findings in the math verification paradigm we expected the P2p amplitude to be 
enhanced to canonical (compared to non-canonical) finger postures and more strongly so to small finger postures 1-4 than larger postures 6-9 (van den Berg et al., 2021). The second ERP component we expected to be modulated by the canonicity of finger postures was the later endogenous P3 component occurring in a window between approximately 300-500 milliseconds after stimulus presentation with highest amplitude above central-parietal electrodes. In earlier number- and finger posture processing studies enhanced P3 amplitude has been linked to easier stimulus evaluation/categorization processes, likely due to better memory representations of the stimuli evoking the enhanced P3 (Dehaene, 1996; Jiang et al., 2010; Libertus et al., 2007; Soylu et al., 2019; Turconi et al., 2004; van den Berg et al., 2021). In line with our earlier findings, we expect enhanced centro-parietal P3 amplitude to both canonical finger postures 1-4 and 6-9, as indicative of their faster recognition and easier categorization due to their storage in memory.

\subsection{Method}

\section{Participants}

Participants were thirty-one young adults recruited from the student population at Maastricht University (mean age: 21yrs 8mo [SD 1yr 6mo]; 5 males). All adults provided written informed consent after being informed about the study, and participants received university course credits for their participation. The faculty's local Ethical Committee approved all study procedures (ERCPN_RP2027_2018_34). All procedures were in accordance with the Declaration of Helsinki.

\section{Procedure}

Testing took place in dedicated EEG labs at the university in a soundproof booth and started with explaining the procedure, followed by attachment of the electrodes. Participants first performed the modified computerized Same/Different Task (see below) during which EEG was measured. Before the Same/Different Task, participants underwent a practice session until they reached a performance criterion of $70-80 \%$ correct, followed by a simple, timed, arithmetic paper and pencil test (not reported in the current paper). Instructions were to respond as fast and as accurately as possible and minimize eye blinks and (eye) movements during the tasks. 


\section{Finger-Number Same/Different Task}

In the Finger-Number Same/Different Task, participants were presented with Arabic numerals (either in the numerical range 1-4, or 6-9) in the center of the screen, flanked by hands showing numerosity congruent (same as the Arabic numeral) or numerosity incongruent (different from the Arabic numeral) finger-number patterns. Hands presented either canonical (culture-typical, familiar) or non-canonical (unfamiliar) finger-number configurations (see Figure 5.1 for a full trial, including timing information and with examples of stimuli). The participants' task was to press the left button on a Cedrus RB844 button box as fast and accurate as possible when the finger posture and Arabic digit indicated the same numerosity and the right button in case of both presenting a different numerosity.

The canonical finger configurations use Western European, single-handed conventions (e.g., thumb, index, and middle finger for three). Non-canonical configurations used a similar single-handed convention (e.g., thumb, middle finger, pinky finger) in order to keep the stimuli perceptually similar and not to introduce confounding due to a change in number of hands to represent small numbers 1-4 between canonical and non-canonical conditions. The lower numerical range (1-4) for canonical and non-canonical trials was accompanied by a closed hand (denoting zero), whereas the higher range (6-9) used an open hand (denoting five). Finger representations of number five were excluded since single-handed non-canonical representations of five are not possible.

The Same/Different Task consisted of 288 unique trials (presented in six blocks of 48 trials). To balance the numerical distance and the frequency of numerals and hands, 40 out of 288 trials consisted of a stimulus presenting a numeral in the low (1-4) numerical range paired with finger-number configurations in the high (6-9) range or vice versa. Because of the aim to distinguish between finger-number pattern - Arabic digit comparisons within low (1-4) and high (6-9) numerical ranges (see introduction), these 40 cross-range different trials were excluded from the analysis, which led to $58 \%$ (144) same trials and $42 \%$ (104) different trials. The remaining 248 trials were still balanced to have $50 \%$ canonical/noncanonical and 50\% low/high range trials in same and different conditions. Stimulus presentation was quasi-random, with the restrictions of identical Arabic numerals never being presented directly after each other, and one type of hand stimulus (canonical/noncanonical) never being shown more than four times in a row. All stimuli were presented on a grey background inside a white box at the center of a 19-inch monitor at a viewing distance of $57 \mathrm{~cm}$. 


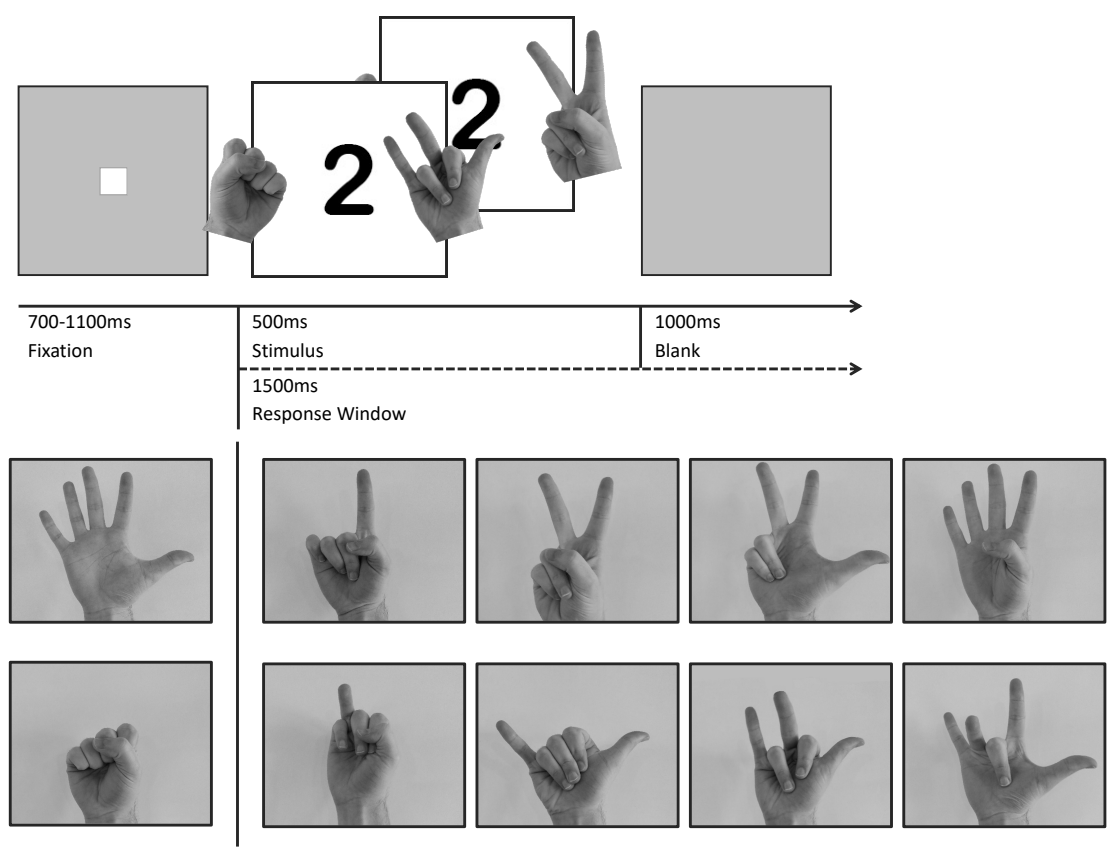

Figure 5.1: An example of a trial with timing parameters from the Same/Different numerical comparison task (top) and the finger-number stimuli used in the experiment (bottom). Due to copyright reasons, the real hand stimuli used in the study (which were drawings) are in this figure replaced by self-made pictures showing the postures. The trials included a variable interval (between 700 and $1100 \mathrm{~ms}$ ), followed by presentation of the stimulus (here two examples of an Arabic numeral flanked by canonical (foreground) or non-canonical (background) finger-number configurations are depicted). The stimulus was presented for $500 \mathrm{~ms}$, after which a blank screen was presented until a response was given or for a maximum duration of $1000 \mathrm{~ms}$. Responses were registered during the whole $1500 \mathrm{~ms}$ interval

\section{EEG/ERP acquisition and analyses}

Electro-Encephalographic (EEG) data were recorded using an elastic EEG-electrode cap (EasyCap; Nellcor-Puritan Bennet, Hayward, CA) with a 38 tin electrode set-up (FP1, FP2, F7, F3, Fz, F4, F8, FC5, FC1, FCZ, FC2, FC6, T7, C3, Cz, C4, T8, CP5, CP1, CPz, $\mathrm{CP} 2, \mathrm{CP} 6, \mathrm{P} 7, \mathrm{P} 3, \mathrm{Pz}, \mathrm{P} 4, \mathrm{P} 8, \mathrm{PO} 7, \mathrm{O} 1, \mathrm{Oz}, \mathrm{O} 2, \mathrm{~A} 2, \mathrm{AFZ}, \mathrm{A} 1)$. The data were filtered online at $0.01-225 \mathrm{~Hz}$ and continuously sampled at a rate of $500 \mathrm{~Hz}$ using a BrainAmp amplifier system and software (Brain Products GmbH, Munich, Germany). The AFz electrode served as ground, and the left mastoid (A1) acted as the online reference; the right mastoid (A2) was included as an extra active electrode. Horizontal and vertical EOG was measured by electrodes placed on each eye's outer canthus and above/below the left orbit. All electrode impedances were kept below $5-10 \mathrm{kOhm}$ and were frequently checked during testing. 
For offline analyses of the EEG data, Matlab2019a/EEGlab2019/ERPLABv8.0.0 software was used. The data was first resampled to $250 \mathrm{~Hz}$ and re-referenced to the average signal. Next, a band-pass filter of $0.1 \mathrm{~Hz}$ to $70 \mathrm{~Hz}$ was applied to the data before execution of Independent Component Analysis (ICA) for removal of horizontal eye movements and blinks. The ocular artifact-free data was filtered using a $30 \mathrm{~Hz}$ Low-pass filter, after which the data was epoched (based on the Hand-configuration-quantity and symbol-quantity event-codes, excluding epochs with incorrect responses) into $300 \mathrm{~ms}$ pre-stimulus and $1500 \mathrm{~ms}$ post-stimulus time windows. Baseline correction was performed using the prestimulus $-300-0 \mathrm{~ms}$ interval. Next, an automatic artifact detection procedure was applied to the remaining epochs (only at electrodes PO7, PO8, and $\mathrm{Pz}$ that were included in the analyses), rejecting trials with activity exceeding a $\pm 75 \mu \mathrm{V}$. An average of $0.98 \%(0-25 \%)$ of the total number of trials in the task were rejected based on artifacts. Participants were only included in the subsequent analyses if at least $50 \%$ of trials for each bin (each combination of Canonicity, Same/Different, and Numerical Range) remained for averaging (after removal of incorrectly responded and artifact trials); since ERP data of all participants fulfilled this criterion, all were included in the analysis.

The choice of electrodes and time-windows to be included in the statistical analyses was based on previous ERP/MEG studies investigating the neural processing of finger- or hand gestures as reviewed in the introduction (Avikainen et al., 2003; Nakamura et al., 2004; Soylu et al., 2019) and visual inspection of the grand average ERP signals and topography maps in the different conditions. This led to the analyses being focused on (P2p) activity in two time windows (from 220-275 ms and 280-350 ms) at left and right Parietal-Occipital electrodes (PO7 and PO8; see Figures 3 and 4 for Grand Average ERPs and topo-maps). The P2p amplitude data was analyzed in these two separate time windows because of the latency shift that is clearly visible between finger patterns 6-9 and 1-4, with P2p peaking later for finger-number configurations 1-4. Mean P3 amplitude was computed in a window from 400-600 ms window at the Pz electrode (see Figure 5.5). Mean amplitude values within these time windows were entered in the statistical analyses.

\section{Statistical Analysis}

To analyze the dependent measures RT, P2p amplitude (in two time windows) and P3 amplitude, Linear Mixed-effects models were constructed. For the accuracy data, Generalized Estimation Equation (GEE) models were constructed because of severe normality violations. All models included within-subject factors Canonicity (2 levels: Canonical and Non-Canonical finger-number configurations), Congruency (2 levels: 
Congruent and Incongruent, with Arabic digit and finger pattern respectively indicating the same or a different numerosity), Numerical Range (2 levels: Low [numbers 1, 2, 3, 4] and High [numbers 6, 7, 8, 9]). The ERP amplitude analyses for the P2p windows included an additional factor Hemisphere (2 levels: Left and Right).

Assumptions were checked using a Compound Symmetry covariance structure, removing single observations (single data-points) as outliers if residuals exceeded three standard deviations. A single outlier was removed for the behavioral Accuracy data; no observations were removed from the ERP and RT analyses. Assumptions of normality and homoscedasticity were met in all analyses. For dependent variables RT and ERP amplitude, various covariance matrices were compared using AIC to determine the best fitting model. For the RT models, an Unstructured matrix showed the best fit. For ERP analyses, both the early and late P2p models showed the best fit with the Unstructured matrix, while the P3 (400-600ms) data fitted best with the Toeplitz covariance matrix. All models were subsequently reduced by removing non-significant interactions.

\subsection{Results}

\section{Behavioral results}

RT. The response time analysis yielded significant main effects of Canonicity $(F[1,30]=$ $14.04, p=.001)$, Congruency $(F[1,30]=51.55, p<.001)$, and numerical Range $(F[1,30]$ $=176.87, p<.001)$, and three two-way higher-order interactions between Canonicity*Congruency $(F[1,30]=8.34, p=.007)$, Canonicity*Range $(F[1,30]=7.19, p$ $=.012)$, and Range*Congruency $(F[1,30]=12.39, p=.001)$. The means and SD's in all conditions are presented in Table 5.1 and the three two-way interactions on RT are visualized in Figure 5.2. Further testing of the Canonicity*Congruency interaction showed a statistically significant canonicity effect on numerosity congruent trials, where finger-pattern and Arabic digit represent the same number $\left(\mathrm{M}_{\mathrm{Diff}}=21.83 \mathrm{~ms} ; t(30)_{\mathrm{NCan}-\mathrm{Can}}\right.$ $=4.71, p<.001$ ), with faster comparison times for canonical than non-canonical finger patterns. No statistically significant canonicity effect was found on numerosity incongruent trials $\left(\mathrm{M}_{\text {Diff }}=5.49 \mathrm{~ms} ; t(30)_{\mathrm{NCan}-\mathrm{Can}}=1.19, p=.242\right)$. Further testing of the Canonicity*numerical Range interaction showed that participants' finger-pattern - Arabic digit comparisons were faster on canonical than non-canonical trials in the low (1-4) numerical range $\left(\mathrm{M}_{\text {Diff }}=24.97 \mathrm{~ms} ; t(30)_{\mathrm{NCan}-\mathrm{Can}}=4.59, p<.001\right)$, but there was no canonicity effect for finger patterns presenting a number in the high (6-9) range $\left(\mathrm{M}_{\text {Diff }}=\right.$ $\left.2.36 \mathrm{~ms} ; t(30)_{\mathrm{NCan}-\mathrm{Can}}=0.41, p=.683\right)$. Finally, the Range*Congruency interaction 
indicated that whereas statistically significant congruency effects (faster responses on numerosity congruent than incongruent trials) were found for both small (1-4) and large (6-9) finger patterns, the effect was larger in the low numerical range $\left(\mathrm{M}_{\text {Diff }}=64.02 \mathrm{~ms}\right.$; $\left.t(30)_{\mathrm{NCan}-\mathrm{Can}}=8.16, p<.001\right)$ than the High numerical range $\left(\mathrm{M}_{\text {Diff }}=30.22 \mathrm{~ms}\right.$; $\left.t(30)_{\mathrm{NCan}-\mathrm{Can}}=3.59, p=.001\right)$. Hence, the reaction time data showed a processing advantage for congruent and low-range canonical hand gestures (see Figure 5.2).

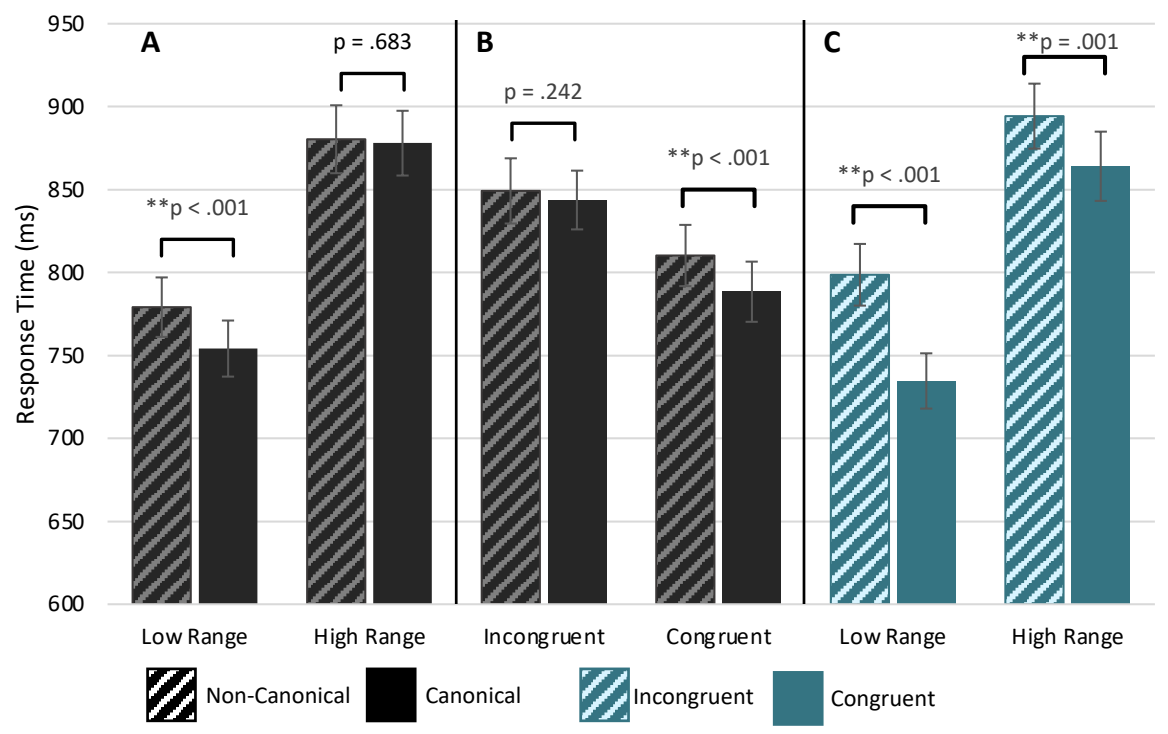

Figure 5.2: Reaction Time results. Panel A shows the Range*Canonicity interaction, with faster finger-pattern digit comparison times when finger patterns were canonical than non-canonical (facilitation effect) only for the low (1-4) numerical range. Panel B shows the Congruency*Canonicity interaction, with canonicity effects (faster responses in the case of canonical finger patterns) occurring only in the condition where finger pattern and digit indicated the same numerosity. Panel $\mathrm{C}$ shows the Range*Congruency interaction, with a larger same - different effect (faster comparisons on same than different trials) to stimuli where finger patterns/digits represented a numerosity in the low (1-4) number range.

Accuracy. The analysis of accuracy only showed significant main effects of Congruency $\left(\chi_{1}^{2}=6.15, p=.013\right)$ and numerical Range $\left(\chi_{1}^{2}=29.38, p<.001\right)$; for means see Table 5.1. Comparison accuracy was higher on congruent trials where finger-pattern and digit presented the same numerosity than on incongruent trials $\left(\left(\mathrm{M}_{\text {Diff }}=1.38 \%\right.\right.$; $\left.t(1)_{\text {Con-Incon }}=2.50, p=.013\right)$, and for finger-number configurations in the low (1-4) compared to the higher (6-9) numerical range $\left(\mathrm{M}_{\text {Diff }}=2.67 \% ; t(1)_{\text {Low-High }}=5.45, p<\right.$ $.001)$ ). None of the other main or interaction effects reached significance (all p-values $>$ $.096)$. 


\section{ERP results}

The behavioral (RT) data only showed a canonicity effect in numerosity congruent (and not incongruent) trials with faster processing times for canonical number-finger configurations (as was also shown in earlier studies; Badets et al., 2010; Di Luca et al., 2006; Di Luca and Pesenti, 2008; Sixtus et al., 2017. Therefore, the results of the following ERP analyses are described separately for numerosity congruent (same) and numerosity incongruent (different) trials, for each component we planned to analyze. As introduced in paragraph 2.4 , the P2p data are analyzed in two time windows to take into account peak latency differences between finger patterns 1-4 and 6-9.

\section{P2 amplitude between $220-275$ ms}

In this early $\mathrm{P} 2 \mathrm{p}$ time-window (see Figure 5.3), numerosity congruent trials showed main effects of Canonicity $(F[1,30]=14.95, p=.001)$ and Hemisphere $(F[1,30]=6.25, p$ $=.018)$ : the $\mathrm{P} 2 \mathrm{p}$ amplitude was larger on Canonical than Non-canonical trials $\left(\mathrm{M}_{\text {Diff }}=\right.$ $\left.0.59 \mu \mathrm{V} ; t(30)_{\text {Can-NCan }}=3.87, p=.001\right)$ and was higher above the right $(\mathrm{PO} 8)$ than left (PO7) parietal-occipital cortex $\left(\mathrm{M}_{\text {Diff }}=1.57 \mu \mathrm{V} ; t(30)_{\text {Right-Left }}=2.50, p=.018\right)$. There were no other statistically significant main or interaction effects (all p's > .175).

Numerosity incongruent trials (see Figure 5.4) showed no significant main or interaction effects of factors Canonicity, numerical Range, or Hemisphere on P2p amplitude in this early time window (all p-values $>$.066).

\section{P2 amplitude between $280-350 \mathrm{~ms}$}

In this late P2p window (see Figure 5.3), numerosity congruent trials showed main effects of Canonicity $(F[1,30]=8.59, p=.006)$, numerical Range $(F[1,30]=48.55, p<.001)$, and Hemisphere $(F[1,30]=4.87, p=.035)$, but none of these factors interacted with each other (all p-values $>.471$ ). The main effect of canonicity showed larger P2p amplitude on Canonical than Non-canonical trials $\left(\mathrm{M}_{\text {Diff }}=0.55 \mu \mathrm{V} ; t(30)_{\text {Can-NCan }}=2.93, p=.006\right)$. The main effect of numerical Range showed larger P2p amplitude on trials showing numbers in the lower (1-4) than the higher (6-9) numerical range $\left(\mathrm{M}_{\text {Diff }}=1.90 \mu \mathrm{V} ; t(30)_{\text {Low-High }}=\right.$ $6.97, p<.001)$, Finally the main effect of Hemisphere showed higher P2p amplitude above the right (PO8) than the left (PO7) parietal-occipital cortex $\left(\mathrm{M}_{\text {Diff }}=1.51 \mu \mathrm{V} ; t(30)_{\text {Right-Left }}\right.$ $=2.21, p=.035$ ). 
Numerosity incongruent trials (see Figure 5.4) only showed a main effect of numerical Range $(F[1,30]=52.73, p=.025)$, which interacted with Hemisphere $(F[1,30]=5.54, p$ $=.025$ ). Further testing of the interaction showed that the numerical range effect (larger $\mathrm{P} 2 \mathrm{p}$ amplitude in response to low range (1-4) than high range (6-9) number stimuli) was larger above the right $\left(\mathrm{M}_{\text {Diff }}=2.42 \mu \mathrm{V} ; t(30)_{\text {Low-High }}=6.90, p<.001\right)$, than the left $\left(\mathrm{M}_{\text {Diff }}\right.$ $\left.=1.41 \mu \mathrm{V} ; t(30)_{\text {Low-High }}=4.31, p<.001\right)$ parietal-occipital hemisphere. None of the other main or interaction effects reached significance (all p-values $>.191$ ).

\section{P3 amplitude (at Pz) between $400-600 \mathrm{~ms}$}

For numerosity congruent trials (see Figure 5.5) main effects of Canonicity $(F[1,18.51]=$ $5.89, p=.026)$ and numerical Range $(F[1,40.85]=40.33, p<.001)$ were found; P3 amplitude was larger on Canonical than Non-canonical trials $\left(\mathrm{M}_{\text {Diff }}=0.55 \mu \mathrm{V}\right.$; $\left.t(18.51)_{\text {Can-NCan }}=2.43, p=.026\right)$ and P3 amplitude was enhanced when Arabic digits and finger patterns represented numerosities in the lower (1-4) numerical range than the higher (6-9) range $\left(\mathrm{M}_{\text {Diff }}=1.30 \mu \mathrm{V} ; t(40.85)_{\text {Low-High }}=6.35, p<.001\right)$. The Canonicity*Range interaction was not significant $F[1,14.50]=1.03, p=.326)$.

Numerosity incongruent trials (see Figure 5.5) only showed a significant main effect of numerical Range $(F[1,48.04]=6.35, p=.015)$; P3 amplitude was larger on trials in which Arabic-digits - finger patterns represented a numerosity in the low (1-4) than the high (6-9) numerical range $\left(\mathrm{M}_{\text {Diff }}=0.74 \mu \mathrm{V} ; t(48.04)_{\text {Low-High }}=2.52, p=.015\right)$. No significant main effect of Canonicity $(F[1,25.51]=0.42, p=.524)$ or Canonicity*Range interaction $(F[1,30.36]=0.20, p=.657)$ was found. 


\section{Congruent Trials showing the P2p Component at PO7 and PO8}
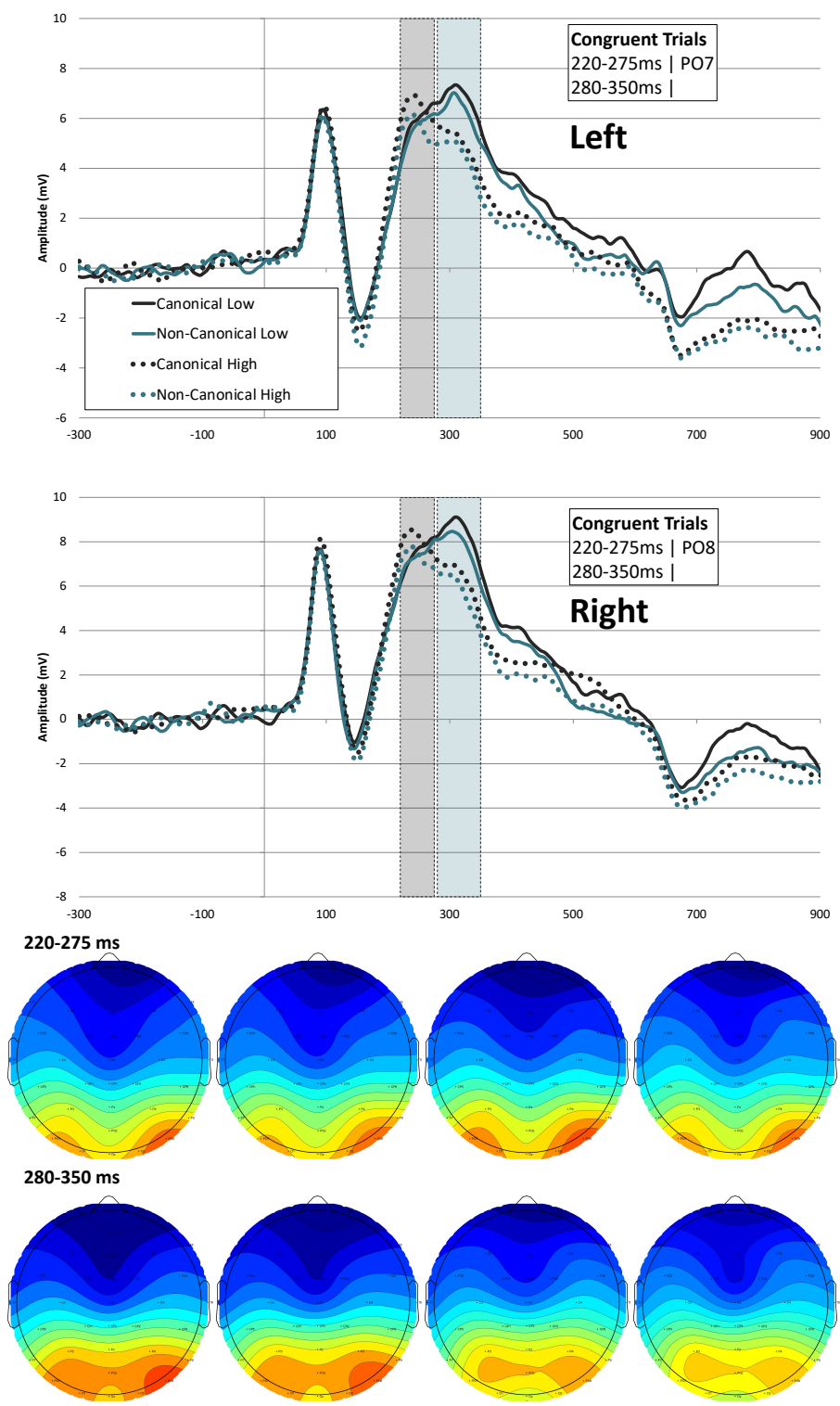

$-6$

Non-Canonical Low

Canonical High

Non-Canonical High $-4$ $-2$ 0 4 8

Figure 5.3: Grand Average ERPs on numerosity Congruent trials with the grey and red colored bars respectively indicating the early (220-275 ms) and late (280-350 ms) P2p scoring windows. The two bottom rows present the topography maps showing the distribution of $\mathrm{P} 2 \mathrm{p}$ activity across the scalp in the two time-windows (top row the early P2p time-window and bottom row the later P2p time window) in the four Canonicity-Range categories: Canonical-Low, Non-Canonical-Low, Canonical-High, Non-Canonical-High. 


\section{Incongruent Trials showing the P2p Component at PO7 and PO8}
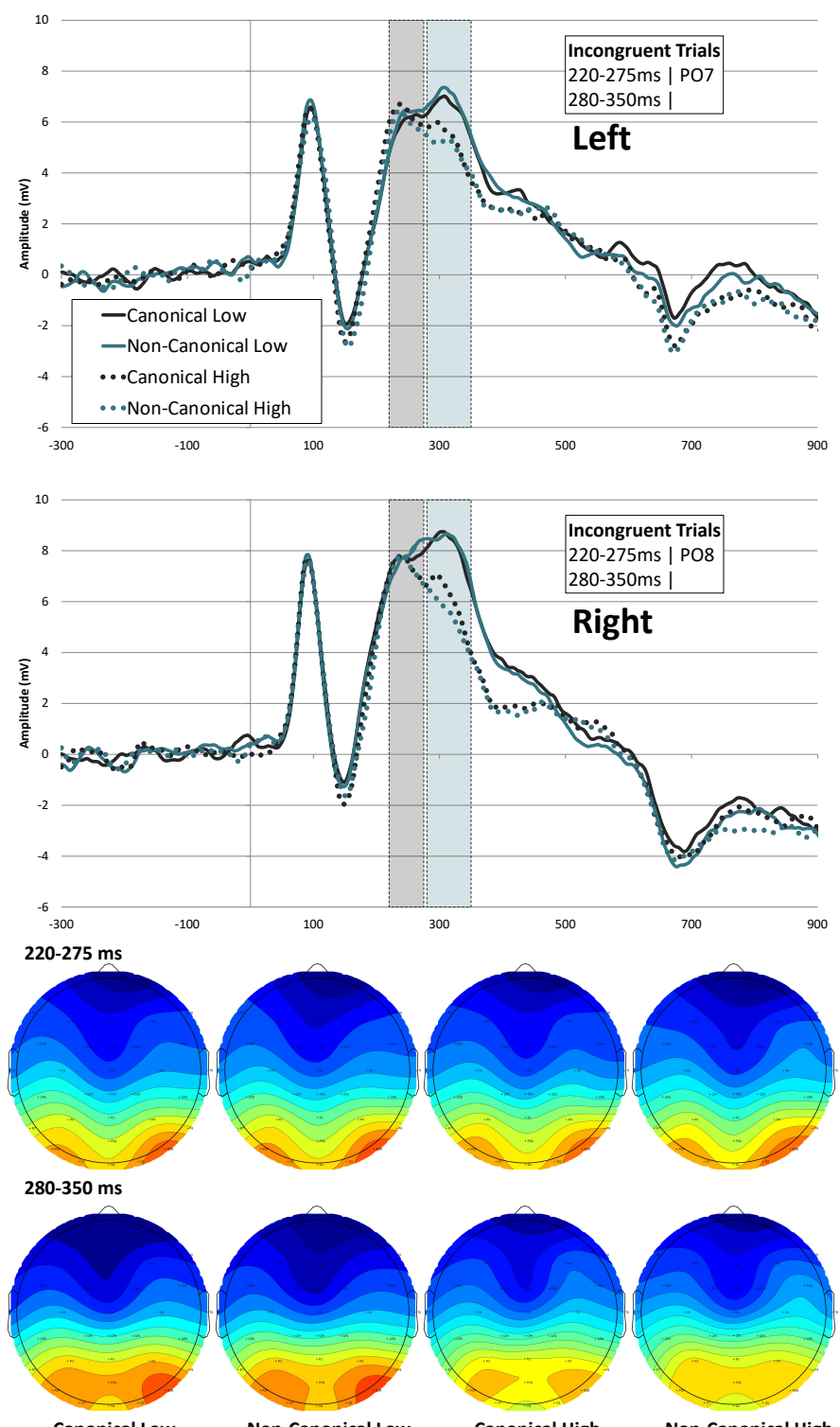

$-6$ $-4$ $-2$

Non-Canonical Low

Canonical High

Non-Canonical High

Figure 5.4: Grand Average ERPs on numerosity Incongruent trials with the grey and red colored bars respectively indicating the early (220-275 ms) and late (280-350 ms) P2p scoring windows. The two bottom rows present the topography maps showing the distribution of $\mathrm{P} 2 \mathrm{p}$ activity across the scalp in the two time-windows (top row the early P2p time-window and bottom row the later P2p time window) in the four Canonicity-Range categories: Canonical-Low, Non-Canonical-Low, Canonical-High, Non-Canonical-High. 


\section{Congruent and Incongruent Trials showing the P3 Component at Pz}
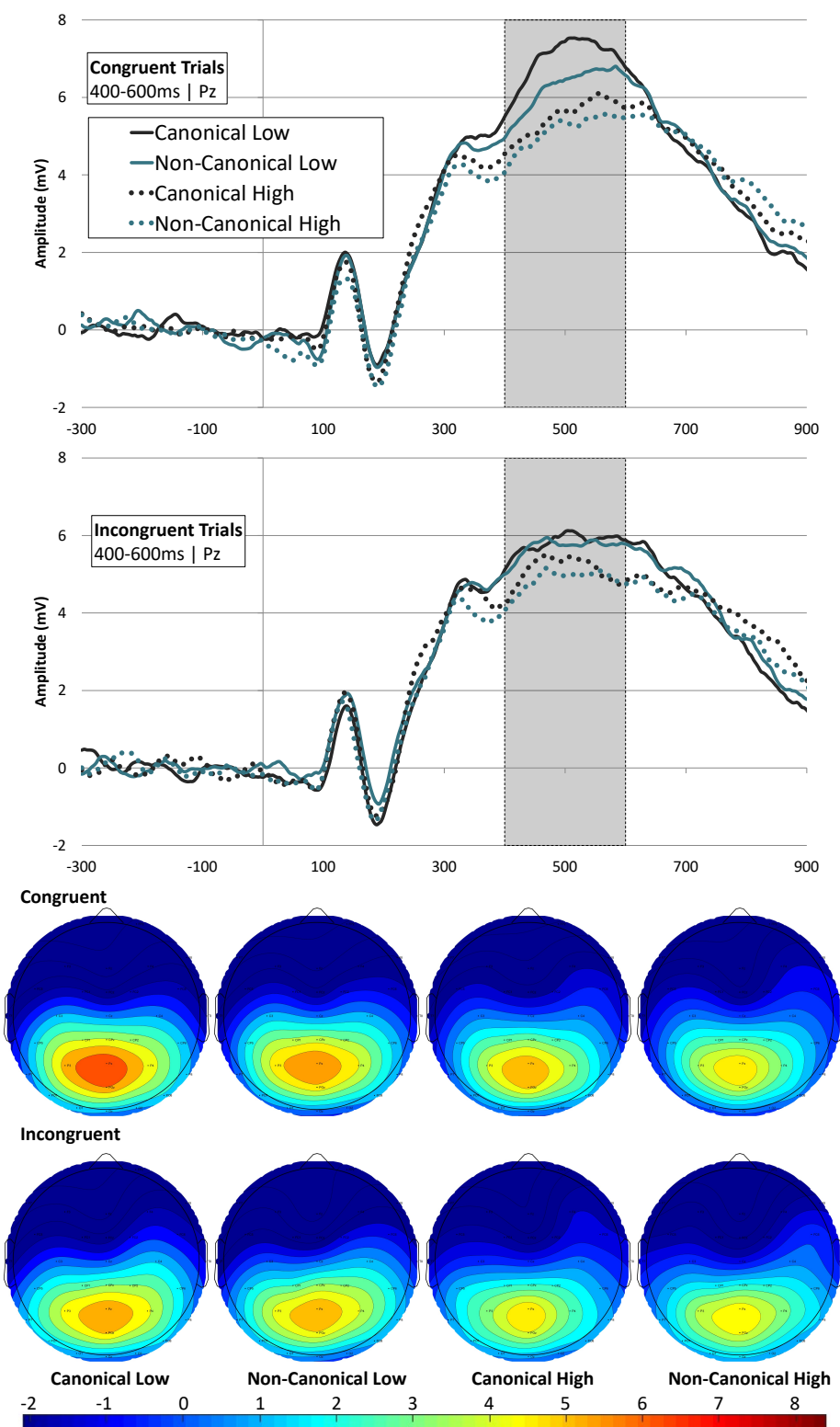

Figure 5.5: Grand Average ERPs in numerosity congruent (top ERP figure) and numerosity incongruent (bottom ERP figure) conditions, with the grey colored bar indicating the 400-600 ms time window across which mean P3 amplitude was computed. Topography maps show the mean P3 activity across the scalp in the 400-600 ms time-window in numerosity congruent (top row) and incongruent (bottom row) conditions in the four Canonicitynumerical Range categories: Canonical-Low, Non-Canonical-Low, Canonical-High, Non-Canonical-High. 
Table 5.1: Means and Standard Errors (between brackets) for behavioral and ERP data in the four CanonicityRange conditions separated for Solution Correctness and hemisphere.

\begin{tabular}{|c|c|c|c|c|c|c|}
\hline & & & Non-Canon & & Canonical & \\
\hline \multirow{4}{*}{$\begin{array}{l}\text { Reaction Time } \\
\text { (milliseconds: ms) }\end{array}$} & \multirow{2}{*}{ Congruent } & Low Range & \multicolumn{2}{|c|}{$751.19(17.32)$} & \multicolumn{2}{|c|}{$718.05(16.72)$} \\
\hline & & High Range & \multicolumn{2}{|c|}{$869.32(21.10)$} & \multicolumn{2}{|c|}{$858.79(21.00)$} \\
\hline & \multirow{2}{*}{ Incongruent } & Low Range & \multicolumn{2}{|c|}{$807.04(19.62)$} & \multicolumn{2}{|c|}{$790.23(17.87)$} \\
\hline & & High Range & \multicolumn{2}{|c|}{891.37 (20.68) } & \multicolumn{2}{|c|}{897.18 (19.13) } \\
\hline \multirow{4}{*}{$\begin{array}{l}\text { Accuracy } \\
(\% \text { correct })\end{array}$} & \multirow{2}{*}{ Congruent } & Low Range & \multicolumn{2}{|c|}{$94.28 \%(0.82 \%)$} & \multicolumn{2}{|c|}{$96.47 \%(0.65 \%)$} \\
\hline & & High Range & \multicolumn{2}{|c|}{$92.34 \%(0.99 \%)$} & \multicolumn{2}{|c|}{$93.25 \%(0.75 \%)$} \\
\hline & \multirow{2}{*}{ Incongruent } & Low Range & \multicolumn{2}{|c|}{$93.65 \%(0.95 \%)$} & \multicolumn{2}{|c|}{$94.71 \%(0.74 \%)$} \\
\hline & & High Range & \multicolumn{2}{|c|}{$91.32 \%(0.93 \%)$} & \multicolumn{2}{|c|}{$91.16 \%(0.94 \%)$} \\
\hline \multirow{6}{*}{$\begin{array}{l}\text { P3 amplitude } \\
(\mu \mathrm{V}) \text { at } \mathrm{Pz} \text { in } \\
400-600 \mathrm{~ms} \\
\text { time window }\end{array}$} & \multirow{2}{*}{ Congruent } & Low Range & & $6.25(0.55)$ & & $7.00(0.55)$ \\
\hline & & High Range & & $5.15(0.55)$ & & $5.50(0.55)$ \\
\hline & \multirow{2}{*}{ Incongruent } & Low Range & & $5.70(0.55)$ & & $5.73(0.55)$ \\
\hline & & High Range & & $4.85(0.55)$ & & $5.10(0.55)$ \\
\hline & & & \multicolumn{2}{|c|}{ Non-Canonical } & \multicolumn{2}{|l|}{ Canonical } \\
\hline & & & Left & Right & Left & Right \\
\hline \multirow{4}{*}{$\begin{array}{l}\text { P2p- amplitude }(\mu \mathrm{V}) \\
\text { in early }(220-275 \mathrm{~ms}) \\
\text { time window } \\
\text { at PO7 \& PO8 }\end{array}$} & \multirow{2}{*}{ Congruent } & Low Range & $5.53(0.94)$ & $7.10(0.95)$ & $6.11(0.94)$ & $7.69(0.95)$ \\
\hline & & High Range & $5.75(0.90)$ & $7.33(0.89)$ & $6.34(0.89)$ & $7.91(0.88)$ \\
\hline & \multirow{2}{*}{ Incongruent } & Low Range & $6.03(0.91)$ & $7.29(0.87)$ & $6.01(0.88)$ & $7.27(0.86)$ \\
\hline & & High Range & $5.89(0.91)$ & $7.14(0.85)$ & $5.87(0.90)$ & $7.12(0.86)$ \\
\hline \multirow{4}{*}{$\begin{array}{l}\text { P2p- amplitude }(\mu \mathrm{V}) \\
\text { in late }(280-350 \mathrm{~ms}) \\
\text { time window } \\
\text { at PO7 \& PO8 }\end{array}$} & \multirow{2}{*}{ Congruent } & Low Range & $6.29(0.87)$ & $7.90(0.96)$ & $6.84(0.89)$ & $8.45(0.96)$ \\
\hline & & High Range & $4.49(0.84)$ & $5.90(0.81)$ & $5.04(0.86)$ & $6.45(0.84)$ \\
\hline & \multirow{2}{*}{ Incongruent } & Low Range & $6.83(0.88)$ & $8.18(0.94)$ & $6.87(0.81)$ & $8.21(0.91)$ \\
\hline & & High Range & $5.42(0.85)$ & $5.76(0.83)$ & $5.46(0.81)$ & $5.80(0.78)$ \\
\hline
\end{tabular}

Values shown are estimated marginal means with Standard Errors. I Low Range $=$ average of 1, 2, 3 \& 4; High Range $=$ average of $6,7,8$ \& 9 


\subsection{Discussion}

The current study investigated whether culture-typical (canonical) finger-number configurations representing numerosities 1-4 or 6-9 have facilitating effects on the processing of Arabic digits in a same/different (numerosity congruent/incongruent) comparison task collecting behavioral and Event-Related Potential (ERP) data.

\section{Effects of the canonicity of finger-number gestures on number comparison performance}

A significant canonicity*congruency interaction effect on reaction time showed that participants were faster in deciding that a simultaneously presented Arabic digit and a finger-number pattern represented the same numerosity when finger-number gestures were canonical (culture-typical familiar patterns) as compared to when they were non-canonical (unfamiliar pattern). When the numerosity indicated by the Arabic digit and finger patterns did not match (i.e., were numerosity incongruent), number comparison times did not differ in a statistically significant way between canonical and non-canonical finger postures. As reviewed in the introduction, similar facilitating effects of culture-typical (canonical) number gestures on reaction time have been reported in other number processing tasks (Di Luca et al., 2006; Di Luca et al., 2010; Di Luca and Pesenti, 2008; Sixtus et al., 2017; Soylu et al., 2019; van den Berg et al., 2021). The present study found that canonical finger-number gestures only facilitated finger gesture - digit comparison in number congruent conditions; this is in line with findings in two prior studies Di Luca et al. $(2006 ; 2010 ; 2008)$ suggested that these processing benefits of cardinal canonical finger-number gestures are due to their storage in long-term memory, allowing for faster recognition and retrieval of the numerosity that they represent.

The current study further investigated this by also measuring P2p and P3 ERP components that have in prior studies been linked to different stages of number semantic processing. A P2p component localized mainly to right intraparietal regions (Dehaene, 1996; Hyde and Spelke, 2012), has in previous number processing studies been shown to be sensitive to numerical manipulations such as numerical distance effects. This P2p component was therefore suggested to index an early numeric semantic processing stage associated with the automatic activation of non-symbolic magnitude information associated with the to-be-processed number stimulus (Dehaene, 1996; Hyde and Spelke, 2012; Libertus et al., 2007; Temple and Posner, 1998). Numerical distance effects have also been found on the later endogenous parietal P3 component, which have been 
suggested to index domain-general processes related to categorical decision making (Donchin, 1981) or explicit memory recognition or retrieval (Friedman and Johnson, 2000).

\section{Effects of the canonicity of finger-number gestures on ERPs}

The present ERP results showed statistically significant main canonicity effects of the finger-number patterns on the amplitude of both P2p and P3-ERP components, but, in parallel with the reaction time findings, these effects were only present when the finger pattern and the Arabic digit showed the same number (i.e., were congruent in number identity). These ERP canonicity effects in numerical congruent trials entailed higher brain activity (higher P2p and P3 amplitudes) elicited by canonical than non-canonical number-finger patterns. Below we will further discuss the functional meaning of these ERP results.

The P2p in our study showed higher activation above the right than the left parietaloccipital cortex. A similar right-lateralized P2p was first reported by Dehaene (1996) in a number comparison task that required adults to decide whether the magnitude indicated by number symbols 1-9 (words and digits) was smaller/larger than the internal reference number five. In their study, the P2p amplitude was modulated by the numerical distance between the target and reference number, with $\mathrm{P} 2 \mathrm{p}$ amplitude increasing when numerals were closer to the referent, and thus less easy to discriminate due to overlapping analog representations on the mental number line. On the basis of this, Dehaene concluded that the $\mathrm{P} 2 \mathrm{p}$ represents activation associated with the processing stage at which one has first access to the numeric codes associated with the number symbols. The increase in P2p amplitude when discriminating digits numerically closer together was attributed to more effortful processing due to their stronger representational overlap and thus more difficult discrimination. In a prior number learning study we (van den Berg et al., 2020) reported a similar modulation of the occipital-parietal, right-lateralized P2p when adults learned to associate new arbitrary symbols to quantities presented by dot arrays. P2p amplitude was increased above right parietal cortex only for novel symbols that one could successfully map onto a quantity (i.e., for those symbols for which one could retrieve a numeric code), supporting the P2p - numeric access/activation view. Because the current task requires similar numerical judgments, the current higher right-lateralized, occipitalparietal early P2p response to canonical than non-canonical finger gestures is tentatively suggested to index fast, automatic, numerical access or activation of associated numeric codes for canonical finger - number patterns, most likely acquired through their frequent 
use in number development and daily life communication. Whereas ERP activity at the scalp cannot be directly linked to their underlying sources, two MEG studies provide further support for a functional link between posterior/parietal activity in a time window overlapping with the P2p as observed in our study, and the automatic activation of semantic information when seeing hand gestures with culturally acquired symbolic meaning, like hand signs for ‘stop' or 'ok' (Möhring et al., 2014; Nakamura et al., 2004).

Further support for the view that the P2p indexes finger-number gestures' more direct access to numerosity meaning, comes from the study of ERP responses to ideographic or ideophonic stimuli. Ideographic or ideophonic stimuli are symbols or sound/words that directly convey a specific meaning, idea, sensation or feeling, such as the Roman numeral III, an emoji, or the word 'shiver'. Two ERP studies Bien et al., 2012, 2013; Lockwood and Tuomainen, 2015 investigated processing differences between ideophones and non-ideophonic control sounds or words. Both studies reported enhanced P2 activity to ideophonic stimuli; in right intra-parietal sulcus (IPS) in the TMS study by Bien et al. (2012), and above right frontal cortex by Lockwood \& Tuomainen (2015). The higher right-parietal P2 activation to ideophonic stimuli in Bien et al (2012) is very similar to our posterior right-hemispheric $\mathrm{P} 2 \mathrm{p}$ response to the canonical finger-number gestures, suggesting that canonical finger-number patterns might have gained a similar iconic/symbolic status in our adults. Lockwood \& Tuomainen (Lockwood and Tuomainen, 2015) interpreted their finding of enhanced P2 activity to ideophonic words as reflecting the recruitment of sensory integration processes between the ideophonic stimulus itself and the sensory information/sensation automatically triggered by it in another modality. In an fMRI study, Holloway et al. (2013) studied processing differences between Arabic numerals and Chinese number ideographs in bilingual English-Chinese participants and bilingual participants with no knowledge of Chinese ideographs. Whereas the Arabic numerals (e.g., 5 or 6) elicited activation of the left intra-parietal sulcus (IPS) in both groups, the Chinese numerical ideographs (e.g., 五 or 六) activated the right IPS, but only in Chinese speakers who had acquired the cultural meaning of these symbols that are primarily used for communicative purposes, like our canonical number-finger gestures. Together, these findings thus support a relation between (right) parietal cortex activation in the P2 time window and early semantic processing of ideographic or iconic symbols, similar to canonical number gestures. 
Canonical finger-number gestures also elicited a higher central-parietal P3 amplitude between 400-600 milliseconds than non-canonical postures only on numerosity-congruent trials corroborating the reaction time results. As reviewed in the introduction, a similarly enhanced parietal P3 in response to canonical (compared to non-canonical) finger-number postures 1-4 was reported in a previous study by Soylu et al., (2019) and to both canonical postures 2-4 and 6-9 in a previous study from our lab using a math verification paradigm (van den Berg et al., 2021). In these two former studies finger-posture and Arabic digit stimuli were presented sequentially whereas in the present study finger-postures and Arabic digits were presented simultaneously within one stimulus, as in Stroop-like tasks. Furthermore, since in Soylu et al. (2019) finger-posture pictures were presented before the digits, the P3 canonicity effect in this study purely reflects processing related to the extraction of the numeric meaning of the finger stimulus. In contrast, in our earlier study (van den Berg et al., 2021) and in the current study the P3 also reflects active numeric comparison processes on the basis of which participants decided whether finger patterns and other numeric stimuli/representations displayed the same or a different quantity. In prior number comparison ERP studies with standard symbolic (words/digits) or nonsymbolic (dot arrays) number stimuli, P3 amplitude modulation has been associated with the confidence of one's number/magnitude categorization/discrimination response (Dehaene, 1996; Libertus et al., 2007), with higher P3 amplitudes associated with easier stimulus categorization and higher response confidence. In memory recognition paradigms, enhancement of P3 amplitude has been related to facilitated memory recognition processes (Friedman and Johnson, 2000) facilitating stimulus classification and decision making and enhancing response confidence (Donchin, 1981; Eimer and Mazza, 2005; Polich, 2003; Verleger et al., 2005; Ye et al., 2019). Based on this prior work, we tentatively conclude that the enhanced P3 response on numerosity-congruent comparison trials showing canonical (versus non-canonical) finger postures could be a manifestation of facilitated decisionmaking processes related to the easier classification of the numerosity conveyed by the familiar canonical finger patterns due to their storage in memory.

\section{Differences in canonicity effects between finger postures representing small versus large numbers}

The present study expands on findings from previous behavioral and ERP finger-number studies by distinguishing behavioral and ERP responses to finger patterns showing smaller (1-4) or larger numbers (6-9), using a paradigm in which finger patterns and Arabic digits are presented simultaneously and have to be actively compared in magnitude. A significant 
canonicity*numerical range interaction effect on reaction time indicated that the behavioral facilitation effect of canonical finger patterns on comparison reaction time was present only when Arabic numbers and finger patterns represented lower numerosities 1-4, whereas for the higher 6-9 range the canonicity effect on reaction time did not reach statistical significance.

One explanation for the finding that the reaction time canonicity effect was stronger for finger postures presenting smaller numbers 1-4, may be that canonical finger gestures have developed stronger associations with numerical meaning in early childhood, because these gestures provide a scaffold for acquiring the numeric meaning of the first four number words (Wynn, 1990, 1992). Such an explanation would also be in line with some recent models and findings that suggest that only the first four number symbols are mapped directly onto corresponding non-symbolic representations of magnitude (Carey and Barner, 2019; Le Corre and Carey, 2007; Reynvoet and Sasanguie, 2016; van den Berg et al., 2020). Fingers might support this initial mapping process of connecting abstract number codes, such as number words and later Arabic digits, to corresponding magnitudes in the outside world, as proposed by previous studies (Gunderson et al., 2015; Jay and Betenson, 2017). Thus, the fact that the mapping process in early childhood starts with numbers $1-4$, and that finger gestures smaller than 5 are likely used more frequently in daily communication, could have led to stronger internalized semantic representations of canonical finger patterns of those first four numbers, facilitating their processing. In a previous number-finger processing ERP study we also compared facilitating effects of small one-hand vs larger two-hand canonical finger postures on number processing but in a math verification task (van den Berg et al., 2021). In this task adult participants had to first mentally compute the solution to a simple addition sum that they had to compare with an afterwards presented correct or incorrect sum solution presented in the form of a picture of canonical or non-canonical finger postures. In this previous study a three-way interaction effect was found on number comparison reaction time between canonicity, numerical range of the finger gestures (2-4 versus 7-9) and whether finger patterns showed a correct or incorrect sum solution. That is, canonical (versus non-canonical) finger patterns 2-4 resulted in faster math verification performance irrespective of whether the sum solution was correct or not, whereas canonical postures 7-9 only facilitated math verification performance when they showed the correct sum solution (were numerosity congruent). In the current task there were only two-way interactions of numerical range with canonicity and congruency-level, indicating that small-range finger postures 1-4 showed stronger canonicity and congruency effects than larger finger postures 6-9, but 
these canonicity and congruency effects did not interact. Whereas further study is needed to determine what is causing these differences, both our former and the current study show evidence for canonical (versus non-canonical) finger patterns having facilitating effects on the processing of/comparison with other representations of number, with facilitation being only present or stronger in numerosity-congruent trials and for one-hand canonical patterns $1-4$.

With respect to $\mathrm{P} 2 \mathrm{p}$ and $\mathrm{P} 3$ components only main effects of numerical range were found at left and right parietal-occipital electrodes from $280 \mathrm{~ms}$ onwards, starting in the late P2p window and extending into the central-parietal P3. The amplitude of the P2p and P3 was higher in response to finger patterns showing smaller numbers 1-4 than to larger numbers 6-9 represented on two hands, irrespective of their canonicity or numerical congruency with the Arabic digits. In contrast to the reaction time results, the small/large numerical range effect on $\mathrm{P} 2 \mathrm{p}$ and $\mathrm{P} 3$ amplitude did not interact with the canonicity effect, suggesting additive effects of canonicity and numerical range. Reaction time results show that it took generally longer to process finger patterns for large numbers represented on two hands than finger patterns representing numbers 1-4 on one hand. In line with this and the previous literature, we speculate that the enhanced late-P2p/P3 amplitude to finger patterns showing small numbers 1-4 (irrespective of their canonicity) reflects the facilitated recognition and categorization of the numerosity that they represent because of the overall easier extraction of the numeric code for small numerosities that can be derived via subitizing, whereas larger numerosities need counting.

In summary, the results discussed above show stronger semantic connections between canonical finger-number gestures and their cardinality in the low number range. Canonical finger-number postures facilitated the processing of Arabic numerals, as shown by faster number comparison responses when they showed the same numerosity. The two ERP components investigated in the current study give more insight into the processes that might underlie this facilitating effect of canonical finger-number gestures on number symbol comparison. A right-lateralized P2p component showed an increased amplitude for canonical compared to non-canonical finger-number postures, which we interpreted as indicative of these postures having faster and automatic access to the semantic number meaning in long-term memory, similar to other non-numeric cultural hand signs or learned ideographic and iconic symbols. The amplitude of the centro-parietal P3 was also enhanced in response to canonical finger-number stimuli on numerosity congruent trials, which we interpreted as facilitated decision making on "same" trials due to their storage in 
memory. Together, these results suggest that canonical finger-number representations facilitate symbolic number comparison in adults through acquired semantic associations that allow for faster access to the numerosities that they represent, thereby facilitating later decision making processes such as in the current numeric (finger gesture - Arabic numeral) comparison task. Throughout development canonical finger-number gestures have likely gained an iconic or ideographic status in memory, due to cross-modal associations between their visual shape and the numerosity they represent. These associations allow for faster recognition of their meaning, thereby facilitating the processing of a matching Arabic numeral in the current task. 


\subsection{References}

Avikainen, S., Liuhanen, S., Schürmann, M., \& Hari, R. (2003). Enhanced extrastriate activation during observation of distorted finger postures. Journal of Cognitive Neuroscience, 15(5), 658-663. https://doi.org/10.1162/jocn.2003.15.5.658 (cited on pages 173,178$)$

Badets, A., Pesenti, M., \& Olivier, E. (2010). Response-effect compatibility of fingernumeral configurations in arithmetical context. Quarterly Journal of Experimental Psychology, 63(1), 16-22. https://doi.org/10.1080/17470210903134385 (cited on pages $170-172,174,181)$

Bien, N., ten Oever, S., Goebel, R., \& Sack, A. T. (2012). The sound of size. crossmodal binding in pitch-size synesthesia: A combined tms, eeg and psychophysics study. NeuroImage, 59(1), 663-672. https://doi.org/10.1016/j.neuroimage.2011.06.095 (cited on page 189)

Bien, N., ten Oever, S., Goebel, R., \& Sack, A. T. (2013). Corrigendum to 'the sound of size' crossmodal binding in pitch-size synesthesia: A combined tms, eeg and psychophysics study. NeuroImage, 72, 325. https://doi.org/10.1016/j.neuroimage. 2012.12.006 (cited on page 189)

Butterworth, B. (1999). The mathematical brain (illustrate). Macmillan. (Cited on pages 170,171$)$.

Carey, S., \& Barner, D. (2019). Ontogenetic origins of human integer representations. Trends in Cognitive Sciences, 23(10), 823-835. https://doi.org/10.1016/j.tics. 2019.07.004 (cited on page 191)

Dehaene, S. (1996). The organization of brain activations in number comparison: Event-related potentials and the additive-factors method. Journal of Cognitive Neuroscience, 8(1), 47-68. https://doi.org/10.1162/jocn.1996.8.1.47 (cited on pages $174,175,187,188,190)$

Di Luca, S., Granà, A., Pesenti, M., Semenza, C., \& Seron, X. (2006). Finger-digit compatibility in arabic numeral processing. Quarterly Journal of Experimental Psychology, 59(9), 1648-1663. https://doi.org/10.1080/17470210500256839 (cited on pages $170,173,174,181,187$ )

Di Luca, S., Lefèvre, N., \& Pesenti, M. (2010). Place and summation coding for canonical and non-canonical finger numeral representations. Cognition, 117(1), 95-100. https://doi.org/10.1016/j.cognition.2010.06.008 (cited on pages 171, 173, 174, 187) 
Di Luca, S., \& Mauro, P. (2011). Finger numeral representations: More than just another symbolic code. Frontiers in Psychology, 2(NOV), 272. https://doi.org/10.3389/ fpsyg.2011.00272 (cited on pages 170-172)

Di Luca, S., \& Pesenti, M. (2008). Masked priming effect with canonical finger numeral configurations. Experimental Brain Research, 185(1), 27-39. https://doi.org/10. 1007/s00221-007-1132-8 (cited on pages 170, 171, 173, 174, 181, 187)

Donchin, E. (1981). Surprise!...surprise? Psychophysiology, 18(5), 493-513. https://doi. org/10.1111/j.1469-8986.1981.tb01815.x (cited on pages 173, 188, 190)

Eimer, M., \& Mazza, V. (2005). Electrophysiological correlates of change detection. Psychophysiology, 42(3), 328-342. https://doi.org/10.1111/j.1469-8986.2005. 00285.x (cited on page 190)

Fischer, M. H. (2012). A hierarchical view of grounded, embodied, and situated numerical cognition. Cognitive Processing, 13(1 SUPPL), 161-164. https://doi.org/10.1007/ s10339-012-0477-5 (cited on page 171)

Fischer, U., Moeller, K., Bientzle, M., Cress, U., \& Nuerk, H. C. (2011). Sensori-motor spatial training of number magnitude representation. Psychonomic Bulletin and Review, 18(1), 177-183. https://doi.org/10.3758/s13423-010-0031-3 (cited on page 170)

Friedman, D., \& Johnson, R. (2000). Event-related potential (erp) studies of memory encoding and retrieval: A selective review. Microscopy Research and Technique, 51(1), 6-28 (cited on pages 188, 190).

Fuson, K. C. (1988). Children's counting and concepts of number (Illustrate, Volume 31). Springer New York. https://doi.org/10.1007/978-1-4612-3754-9. (Cited on page 170)

Gunderson, E. A., Spaepen, E., Gibson, D., Goldin-Meadow, S., \& Levine, S. C. (2015). Gesture as a window onto children's number knowledge. Cognition, 144, 14-28. https://doi.org/10.1016/j.cognition.2015.07.008 (cited on page 191)

Holloway, I. D., Battista, C., Vogel, S. E., \& Ansari, D. (2013). Semantic and perceptual processing of number symbols: Evidence from a cross-linguistic fmri adaptation study. Journal of cognitive neuroscience, 25(3), 388-400. https://doi.org/10.1162/ jocn_a_00323 (cited on page 189)

Hyde, D. C., \& Spelke, E. S. (2012). Spatiotemporal dynamics of processing nonsymbolic number: An event-related potential source localization study. Human Brain Mapping, 33(9), 2189-2203. https://doi.org/10.1002/hbm.21352 (cited on pages 174,187 ) 
Jay, T., \& Betenson, J. (2017). Mathematics at your fingertips: Testing a finger training intervention to improve quantitative skills. Frontiers in Education, 2, 22. https: //doi.org/10.3389/feduc.2017.00022 (cited on page 191)

Jiang, T., Qiao, S., Li, J., Cao, Z., Gao, X., Song, Y., Xue, G., Dong, Q., \& Chen, C. (2010). Effects of symbol type and numerical distance on the human eventrelated potential. Neuropsychologia, 48(1), 201-210. https://doi.org/10.1016/j. neuropsychologia.2009.09.005 (cited on page 175)

Knudsen, B., Fischer, M. H., \& Aschersleben, G. (2014). Development of spatial preferences for counting and picture naming. Psychological Research, 79(6), 939-949. https://doi.org/10.1007/s00426-014-0623-z (cited on page 170)

Lafay, A., Thevenot, C., Castel, C., \& Fayol, M. (2013). The role of fingers in number processing in young children. Frontiers in Psychology, 4(7), 488. https://doi.org/ 10.3389/fpsyg.2013.00488 (cited on page 171)

Le Corre, M., \& Carey, S. (2007). One, two, three, four, nothing more: An investigation of the conceptual sources of the verbal counting principles. Cognition, 105(2), 395-438. https://doi.org/10.1016/j.cognition.2006.10.005 (cited on page 191)

Libertus, M. E., Woldorff, M. G., \& Brannon, E. M. (2007). Electrophysiological evidence for notation independence in numerical processing. (Volume 3). https://doi.org/ 10.1186/1744-9081-3-1. (Cited on pages 174, 175, 187, 190)

Lindemann, O., \& Fischer, M. H. (2015). Embodied number processing. Journal of Cognitive Psychology, 27(4), 381-387. https://doi.org/10.1080/20445911.2015.1032295 (cited on page 170)

Lockwood, G., \& Tuomainen, J. (2015). Ideophones in japanese modulate the p2 and late positive complex responses. Frontiers in Psychology, 6, 933. https://doi.org/10. 3389/fpsyg.2015.00933 (cited on page 189)

Maor, E., \& Ifrah, G. (2001). The universal history of numbers: From prehistory to the invention of the computer (Volume 108). Wiley. https://doi.org/10.2307/2695263. (Cited on page 171)

Moeller, K., Fischer, U., Link, T., Wasner, M., Huber, S., Cress, U., \& Nuerk, H. C. (2012). Learning and development of embodied numerosity. Cognitive Processing, 13(1), 271-274. https://doi.org/10.1007/s10339-012-0457-9 (cited on page 170)

Moeller, K., Martignon, L., Wessolowski, S., Engel, J., \& Nuerk, H. C. (2011). Effects of finger counting on numerical development the opposing views of neurocognition and mathematics education. Frontiers in Psychology, 2(NOV), 328. https://doi. org/10.3389/fpsyg.2011.00328 (cited on page 170) 
Möhring, N., Shen, C., \& Neuhaus, A. H. (2014). Spatiotemporal dynamics of early cortical gesture processing. NeuroImage, 99, 42-49. https://doi.org/10.1016/j.neuroimage. 2014.05.061 (cited on page 189)

Nakamura, A., Maess, B., Knösche, T. R., Gunter, T. C., Bach, P., \& Friederici, A. D. (2004). Cooperation of different neuronal systems during hand sign recognition. NeuroImage, 23(1), 25-34. https://doi.org/10.1016/j.neuroimage.2004.04.034 (cited on pages $173,178,189$ )

Polich, J. (2003). Theoretical overview of p3a and p3b. Detection of change (Pages 83-98). Springer US. https://doi.org/10.1007/978-1-4615-0294-4_5. (Cited on page 190)

Proverbio, A. M., \& Carminati, M. (2019). Finger-counting observation interferes with number processing. Neuropsychologia, 131, 275-284. https://doi.org/10.1016/j. neuropsychologia.2019.06.001 (cited on pages 172-174)

Reynvoet, B., \& Sasanguie, D. (2016). The symbol grounding problem revisited: A thorough evaluation of the ans mapping account and the proposal of an alternative account based on symbol-symbol associations. Frontiers in Psychology, 7(OCT), 1581. https://doi.org/10.3389/fpsyg.2016.01581 (cited on page 191)

Siegler, R. S., \& Shrager, J. (1984, January 1). Strategy choices in addition and subtraction: How do children know what to do? https://doi.org/10.1016/S0140-6736(00)020547. (Cited on page 170)

Sixtus, E., Fischer, M. H., \& Lindemann, O. (2017). Finger posing primes number comprehension. Cognitive Processing, 18(3), 237-248. https://doi.org/10.1007/s10339-017-0804-y (cited on pages 171, 174, 181, 187)

Soylu, F., Rivera, B., Anchan, M., \& Shannon, N. (2019). Erp differences in processing canonical and noncanonical finger-numeral configurations. Neuroscience Letters, 705, 74-79. https://doi.org/10.1016/j.neulet.2019.04.032 (cited on pages 171, $173-175,178,187,190)$

Temple, E., \& Posner, M. I. (1998). Brain mechanisms of quantity are similar in 5-year-old children and adults. Proceedings of the National Academy of Sciences of the United States of America, 95(13), 7836-41. https://doi.org/10.1073/pnas.95.13. 7836 (cited on pages 174, 187)

Tran, C., Smith, B., \& Buschkuehl, M. (2017). Support of mathematical thinking through embodied cognition: Nondigital and digital approaches. Cognitive Research: Principles and Implications, 2(1), 1-18. https://doi.org/10.1186/s41235-0170053-8 (cited on page 170) 
Turconi, E., Jemel, B., Rossion, B., \& Seron, X. (2004). Electrophysiological evidence for differential processing of numerical quantity and order in humans. Cognitive Brain Research, 21(1), 22-38. https://doi.org/10.1016/j.cogbrainres.2004.05.003 (cited on page 175)

van den Berg, F. C., de Weerd, P., \& Jonkman, L. M. (2020). Number-related brain potentials are differentially affected by mapping novel symbols on small versus large quantities in a number learning task. Journal of cognitive neuroscience, 32(7), 1263-1275. https://doi.org/10.1162/jocn_a_01546 (cited on pages 188, 191)

van den Berg, F. C., de Weerd, P., \& Jonkman, L. M. (2021). Electrophysiological evidence for internalized representations of canonical finger-number gestures and their facilitating effects on adults' math verification performance. Scientific Reports, 11(1), 1-15. https://doi.org/10.1038/s41598-021-91303-2 (cited on pages $172-175,187,190,191)$

Verleger, R., Jaśkowski, P., \& Wascher, E. (2005). Evidence for an integrative role of p3b in linking reaction to perception. Journal of Psychophysiology, 19(3), 165-181. https://doi.org/10.1027/0269-8803.19.3.165 (cited on page 190)

Wynn, K. (1990). Children's understanding of counting. Cognition, 36(2), 155-193. https: //doi.org/10.1016/0010-0277(90)90003-3 (cited on page 191)

Wynn, K. (1992). Children's acquisition of the number words and the counting system. Cognitive Psychology, 24(2), 220-251. https://doi.org/10.1016/0010-0285(92) 90008-P (cited on pages 170, 191)

Ye, M., Lyu, Y., Sclodnick, B., \& Sun, H. J. (2019). The p3 reflects awareness and can be modulated by confidence. Frontiers in Neuroscience, 13(MAY), 510. https: //doi.org/10.3389/fnins.2019.00510 (cited on page 190) 



\section{The Hand Room}

Standing in this room (at 7) we can see what might have helped children learn the first symbols, the most convenient tool of all: our hands. In many cultures across the globe, humans use their hands for counting and showing quantities. Children can often "show" a number using their hands before they can use the right word. However, how we show a number, which fingers we hold up, is different between cultures. How you show numbers depends on what you were taught by the people around you (such as family, friends, or teachers); in other words, they are culturally dependent. Using hands is not necessary to learn symbols. But hands can be a good tool for young children when they first start connecting number symbols to the quantities they represent.

At numbers 8 and $\mathbf{9}$, we can see different ways of showing the number 3 . Every culture has its way of showing numbers with their hands. There's the German and English three, and some start counting with their thumb while others begin at the pinkie finger. It isn't even limited to hands. In the whole-body number system of some tribes in Papua New Guinea, gestures include the shoulders, elbows, neck, and head to communicate up to twenty-seven different numbers.

For many, the three shown at $\mathbf{8}$ is more familiar than the one shown at $\mathbf{9}$, but both might feel a bit off. When finger numbers are in a cultural pattern, such as the thumb, index, and middle finger to show three, we call those canonical (see the statue at 10). Any other combination of fingers seems strange. You will need to use subitizing (a fast way to 'see' quantities less than four), estimation, or counting to know what someone is trying to tell you. These other ways are called non-canonical finger numbers.

Canonical finger numbers (such as the statue at 10) are special; they are stored in long-term memory. We see them and immediately know how much they mean; they can activate a number concept without counting, subitizing, or estimating. While it might seem that these canonical finger numbers are a different kind of symbol, we have learned they aren't quite like them. Canonical finger numbers lie somewhere in between symbolic and non-symbolic systems; they are called ideographs. Ideographs are visual representations of a concept or idea, such as emojis or pictograms. Canonical finger numbers and older number systems (shown at $1 \mathbf{1}$ ) can be considered ideographs.

We found out that finger numbers still help us process symbols, a remnant of when they might have helped us learn the early numbers. We also learned that finger numbers probably don't have the same role for the larger symbols. Children learn these larger symbols by using rules rather than connecting them to a quantity; there would be no need for hands in that process. It appears that canonical finger numbers can act as a link between natural quantities and symbols (see the display at 12 ), a useful tool for early symbol learning. 


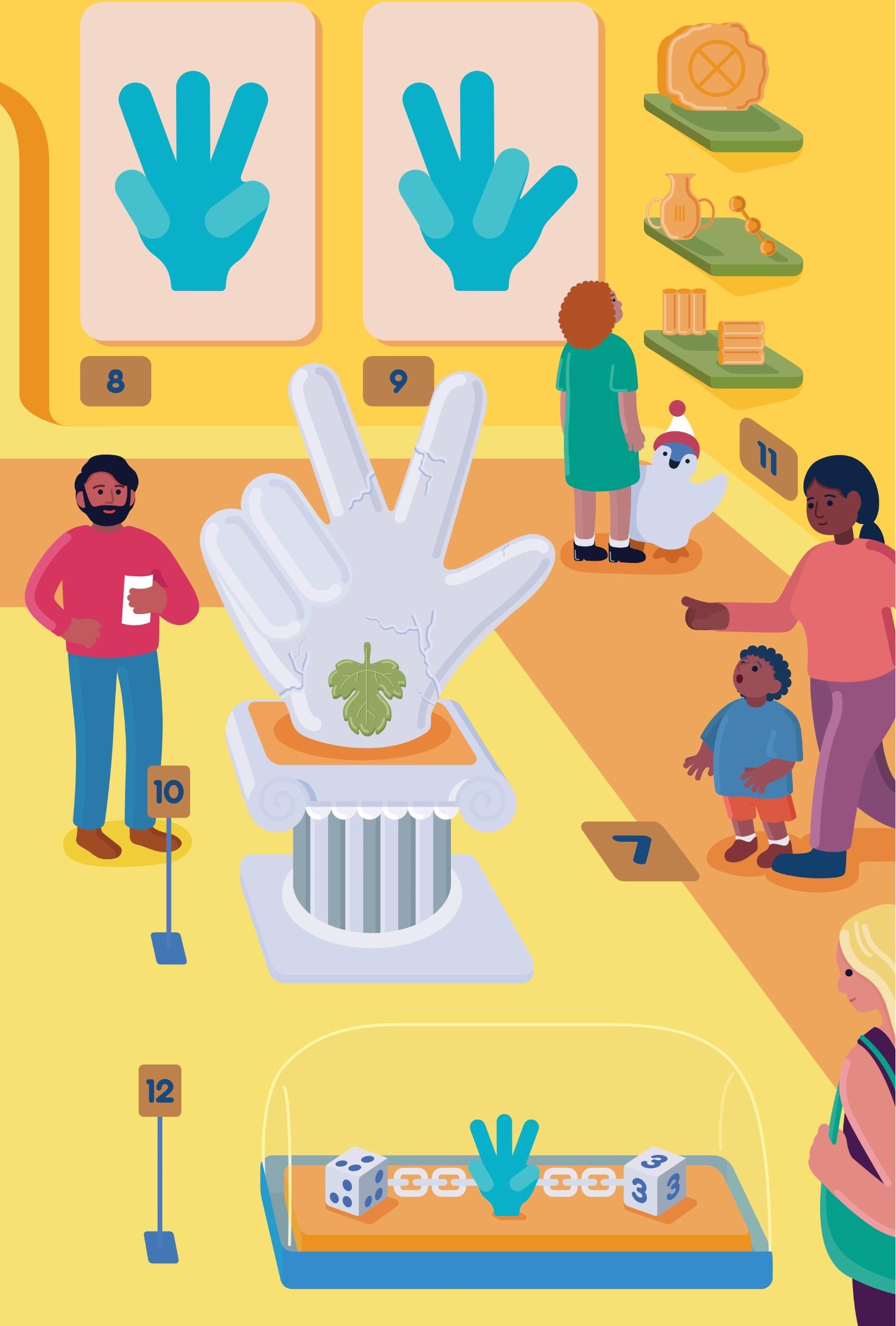





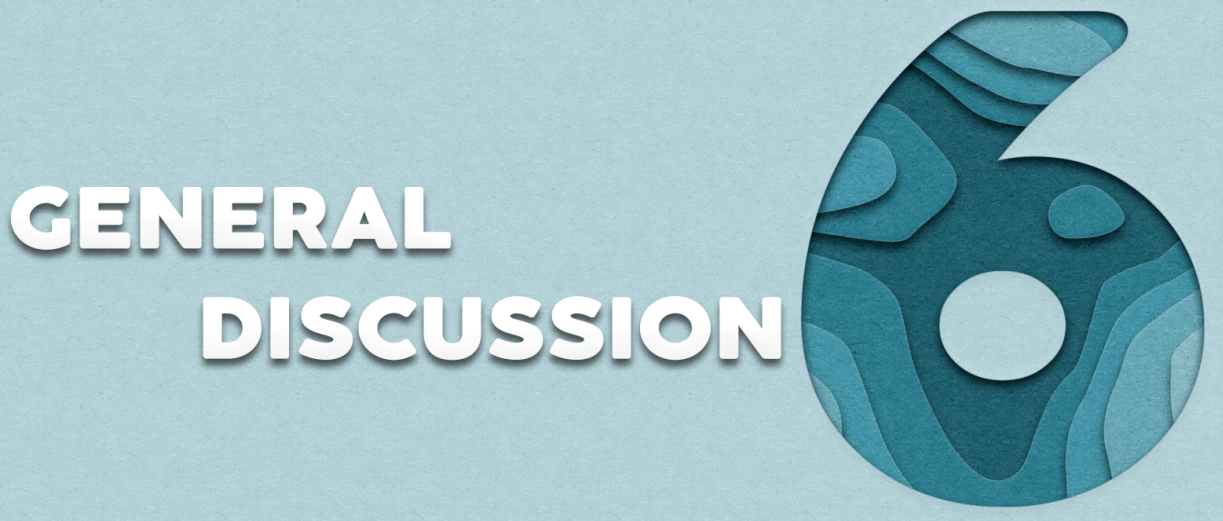

\subsection{General aims}

The studies in this dissertation investigated how number symbols gain their numerical meaning and how such number symbols (including Western standard symbolic stimuli such as Arabic digits, but also finger gestures) are represented and semantically processed in the brain. More specifically, the first two studies described in Chapters 2 and 3 aimed to test the ANS mapping account. These two chapters address whether evidence can be found to support the assumption that number symbols (e.g., Arabic numerals) acquire their numeric meaning by being mapped onto, or associated with, corresponding non-symbolic (e.g., quantity) representations. Additionally, these chapters investigate the differences in mapping between subitizing and non-subitizing ranges. The studies described in Chapters 4 and 5 aimed to investigate the semantic processing of culture-specific (canonical) fingernumber representations and how these might facilitate adult symbolic number processing in the subitizing and non-subitizing ranges.

Whereas the above aims/questions have been partly addressed in prior behavioral work (see Introduction), the studies in the present thesis distinguish themselves from these studies by also measuring event-related brain potentials (ERPs). Whereas behavioral measures such as accuracy and response time are end-state measures, ERPs provide a millisecond view on stimulus processing in the brain from first perception to early and later semantic processing and finally decision-making processes. As is reviewed in the general introduction and the different studies described in the chapters, the number processing-ERP literature has identified various ERP components that are assumed to 
be specifically associated with early and later semantic processing of numeric stimuli (Dehaene, 1996; Hyde and Spelke, 2012; Libertus et al., 2007; Pinel et al., 2001; Szucs and Soltész, 2007; Temple and Posner, 1998; Turconi et al., 2004). In multiple studies in adults and children, the early parietal-occipital P2p-ERP component occurring around 200 milliseconds after stimulus presentation has been shown to be modulated by the numerical distance between two number stimuli in a task requiring magnitude comparisons. This ERP, accordingly, is assumed to reflect the processing stage in which one has first automatic access to domain-specific analog magnitude information (Dehaene, 1996). The later parietal P3-ERP component has been associated with later stages of domaingeneral number processing related to making categorical decisions (Donchin, 1981) or to explicit recognition memory (Friedman and Johnson, 2000), with higher P3 activity evoked by earlier learned/familiar stimuli reflecting higher memory recognition and facilitated stimulus categorization/evaluation. In line with this literature, next to behavioral measures, we found the P2p-ERP component to be present in all four studies of this dissertation. The P2p is included to investigate differences in early semantic processing (automatic access/activation of magnitude information) between the different numeric stimuli used in the studies in Chapters 2-5. The P3-ERP component is additionally included in the studies described in Chapters $\mathbf{4}$ and $\mathbf{5}$ to study differences in memory recognition or stimulus categorization processes between cultural-typical and unfamiliar finger-number gestures, which would provide proof for their internalization and embodiment.

\subsection{The Symbol Grounding Problem: are numerals mapped onto non-symbolic magnitudes?}

Well-developed number representations and understanding is crucial for later numeracy and math development (Duncan et al., 2007; Göbel et al., 2014; Holloway and Ansari, 2009; Siegler and Ramani, 2009). An important milestone in number development is acquiring the symbolic number system, which starts by learning the meaning of number words and number symbols such as Arabic digits. There is, however, a debate in the number processing literature about how number symbols acquire their meaning, known as the symbol grounding problem (Carey, 2009; Dehaene, 2001; Le Corre and Carey, 2007; Leibovich and Ansari, 2016; Piazza, 2010; Reynvoet and Sasanguie, 2016; Siegler, 2016; Spelke, 2017; Sullivan and Barner, 2012). As reviewed in the introduction and the two studies described in Chapters $\mathbf{2}$ and $\mathbf{3}$, different researchers have put forward other propositions of how meaningless number words or symbols gain their numerical 
meaning. One account that long steered number processing research is the ANS mapping account (Dehaene, 2001, 2011; Hersh et al., 1999). The key assumption in this account is that numerical symbols acquire meaning by mapping them directly onto non-symbolic representations of number. This symbolic-non-symbolic mapping account was based on the presence of similar number ratio and numerical distance effects in number magnitude comparison tasks with non-symbolic and symbolic stimuli, pointing to a shared analog representational system (i.e., a mental number line). However, these prior magnitude comparison studies have mostly been performed in adults with fully developed symbolic number systems or in older children at elementary school age who have already learned the meaning of the first ten number words or symbols.

Recent studies in early childhood, when children first learn the meaning of number words and Arabic digits, do not support the assumption that all number symbols are learned via direct symbolic - non-symbolic mapping. Instead, studies in very young children who just started to learn the first number words/symbols suggest that symbols gain their meaning through a combination of direct mapping and symbol-to-symbol associations (Benoit et al., 2013; Odic et al., 2015; Wynn, 1990, 1992). Based on this and their own work, Carey \& Barner (2019) proposed The Exact Algorithms Hypothesis. In line with the previous literature on how children learn the first four numbers (Benoit et al., 2013; Odic et al., 2015; Wynn, 1990, 1992), this hypothesis suggests that these number-words and symbols are directly associated with real-world non-symbolic representations in a oneto-one fashion. These real-world, non-symbolic quantities (e.g., three toys) are identified through subitizing rather than the ANS. Carey \& Barner (2019) posit that larger symbols gain meaning through algorithms once the first four number-words are learned. These algorithms stem from symbol-to-symbol associations (e.g., the ordered nature of number words and the spatial organization of numerals) that allow for strategies that can be used to infer a symbol's numerical meaning (e.g. the counting and successor principles; Carey and Barner, 2019).

In short, the Exact Algorithm Hypothesis states that only symbols in the subitizing range (1-4) would be mapped onto/associated with non-symbolic quantity representations because only quantities up to four can be subitized and are exactly represented. Since number symbols higher than 4-5 cannot be mapped onto the approximate representations for non-subitizable quantities of 4 or higher, they would acquire their meaning via other processes. The studies in Chapters $\mathbf{2}$ and $\mathbf{3}$ addressed this difference between the smaller and larger number ranges using different paradigms and study populations. Chapter 2 
used a paradigm in which adults learned novel number symbols. Chapter $\mathbf{3}$ investigated and compared the longitudinal developmental pattern of magnitude processing in symbolic and non-symbolic number comparison tasks.

\subsubsection{Symbolic - Nonsymbolic mapping during novel number symbol learning in adults}

To study the dynamic symbolic - non-symbolic mapping process during learning, in the study in Chapter 2, the number symbol learning process was mimicked. Number symbol learning was accomplished by letting adults perform a trial-and-error delayed match-to-sample task. The adult participants had to acquire the numeric meaning of novel symbols by associating them with non-symbolic magnitude information presented as dot patterns. Using ERP measures (N1, P2p) in addition to performance measures, we directly tested whether all Arabic digits (1-9) or only digits 1-4 could gain numeric meaning by non-symbolic (magnitude) mapping.

Reaction time and accuracy data provided evidence of one-by-one learning of the first four novel symbols in the subitizing range in ascending order. At first, none of the symbols had a numerical meaning, and deciding if the symbols and dots were equal was at chance level. After several training sessions, direct mapping between non-symbolic and symbols representations only occurred for the subitizeable range (1-4), but not for the higher symbols.

The ERP data confirmed exact symbol - nonsymbolic mapping only taking place for novel symbols representing a numerosity in the subitizing range and not for novel symbols 6-9. First, we found a significant increase in the amplitude of the N1 component over learning sessions. The N1 occurs around 100 milliseconds after stimulus presentation and reflects early perceptual processing. However, the observed N1 increase with learning only occurred when participants saw a symbol preceded by non-matching dots and only in the subitizing range (e.g., seeing three dots followed by any novel symbol $(1,2$, or 4) that was not associated with three). We interpreted these N1 findings as indicating a violation of expectation that only occurred across learning sessions for novel symbols that were successfully associated with a numerosity extracted from the preceding dot-arrays. A participant could only form an expectation if they had an exact numerical representation of the non-symbolic array, which was only possible for quantities less than four using a subitizing strategy. The later P2p component showed decreased amplitude across learning 
sessions only for the first four novel symbols and was not modulated by novel symbols 6-9. Since the $\mathrm{P} 2 \mathrm{p}$ has in the prior number processing literature been associated with the processing stage in which one has first access to magnitude representations, we interpreted this P2p decrease across learning sessions as symbols 1-4 gaining numeric meaning and requiring less cognitive resources to retrieve that meaning.

Taken together, the combined behavioral and ERP findings in Chapter $\mathbf{2}$ show that, based on magnitude information alone, learning the numerical meaning of a symbol can only occur in the subitizing range where exact numerical quantity information can be quickly extracted (subitized). The current task only provided magnitude information that participants used to learn novel symbols. For symbols representing a numerosity outside the subitizing range, we can only conclude that the presented symbols did not gain meaning through direct association with a non-symbolic magnitude when there is no time to use counting to derive the exact quantity of non-symbolic stimuli. The findings of the study in Chapter 2 thus argue against the assumption of the ANS mapping account that all symbols are mapped onto non-symbolic representations. The results fit better with the Exact Algorithms Hypothesis (Carey and Barner, 2019), stating that only symbols 1-4 are exactly mapped onto non-symbolic representations (via the OTS). In contrast, symbols 5 or higher are most likely learned via other processes, like symbol-to-symbol associations.

The results of this learning study are represented in Figure 6.1. The top section displays the situation before learning, where non-symbolic dot-arrays (left column) have associations with the internal number line (middle column). The lines indicate that small quantities (such as three, shown in blue) have an exact representation through subitizing (as demonstrated by a single line connecting it to the internal representation). Larger quantities (such as seven, shown in brown) can only be estimated, shown by the dashed lines connecting to the internal representations for six through nine. The to-be-learned novel symbols are shown in the right column, and since they have no meaning yet, they are connected to all possible internal representations. Seeing a dot-array of three or seven could be any of the nine symbols, indicated by the blue and brown coloring of the symbols. On the bottom is the situation after learning. The repeated association of a symbol with the exact representation of three pruned away the connections between novel symbol 3 and the other internal representation and strengthened the association with the internal representation for three. This association was possible because a non-symbolic array showing three dots only activates a single internal representation. The same happened for symbols 1, 2, and 4. No exact representation was possible for larger arrays, so the 
novel symbols kept the associations with all of the larger internal representations; only the connections with the subitizing range disappeared when those symbols gained a single numerical meaning. 


\section{Before Learning}

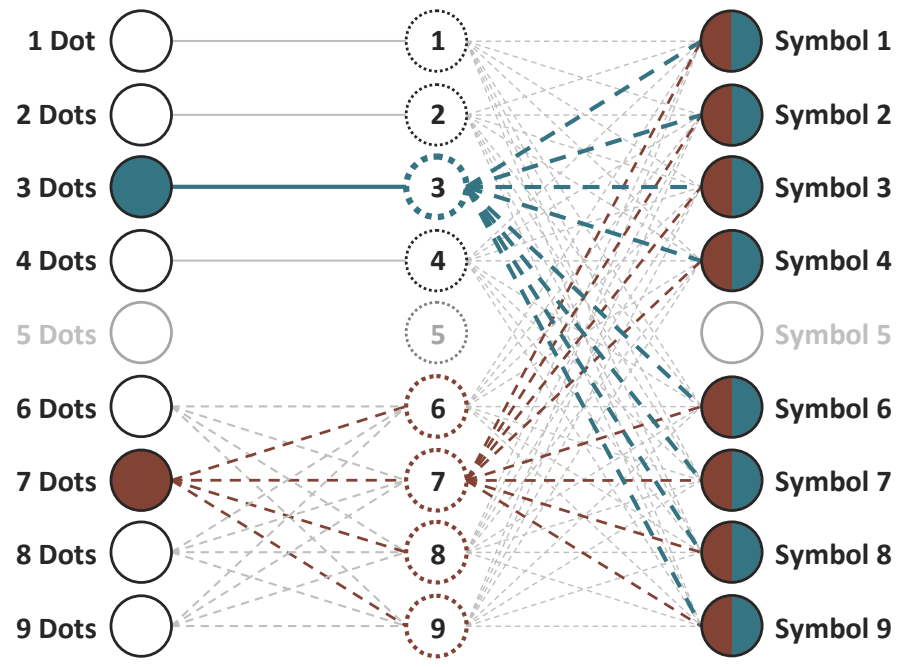

\section{After Learning}

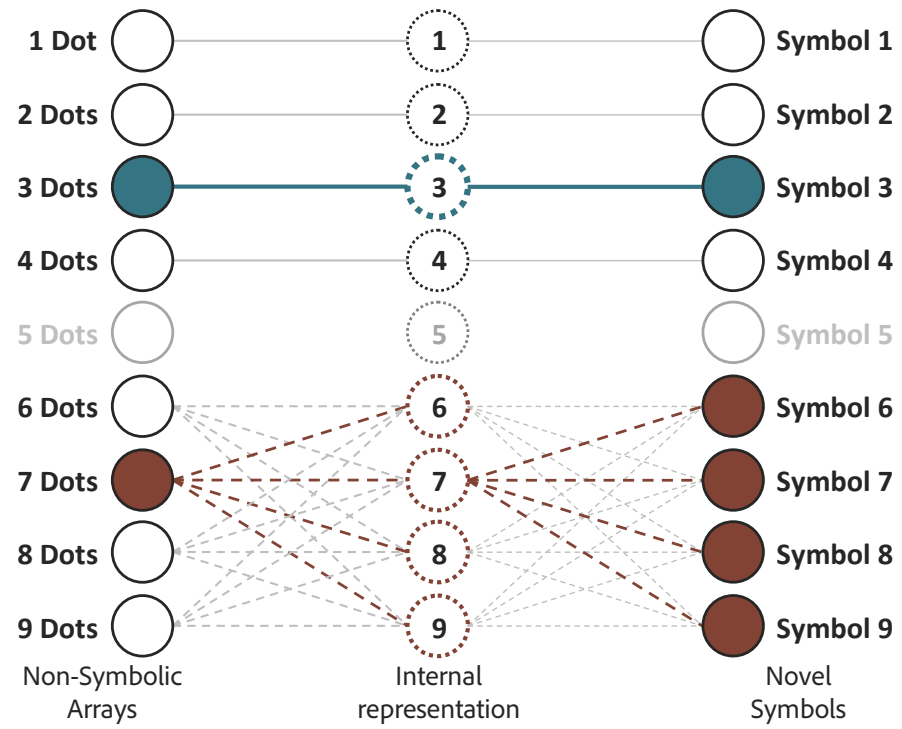

Figure 6.1: schematic representation explaining the possible way in which novel symbolic stimuli (right) are associated with their corresponding non-symbolic stimuli (left) via access to internal abstract magnitude (number line) representations (middle) before and after they have acquired numeric meaning (respectively top and bottom picture). Initially, all symbols could represent any quantity (top), but after learning only the first four symbols have gained an exact numeric meaning via one to-one mapping between symbolic and nonsymbolic number stimuli; bottom picture), whereas this is not the case for numbers 6-9 due to the imprecise estimation of the numerosity of quantities higher than 4 that cannot be subitized, thus not allowing for one-to-one mapping and thus exact representation). "Three" connects directly to symbol three, but "seven" is still connected to all higher symbols. 


\subsubsection{Longitudinal development and symbolic and non-symbolic mapping}

The above discussed learning study mimicked symbol learning in adult participants who already had a fully developed number symbol system. Only a few studies in young children have investigated differences in symbolic - non-symbolic mapping between the smaller subitizing and larger estimation range. Furthermore, prior mapping studies in children were cross-sectional, measuring performance on non-symbolic and symbolic tasks at one time point, not allowing for conclusions about causality, i.e., whether the development of symbolic representations follows that of non-symbolic representations in time (which you would expect in case of symbol-nonsymbolic mapping), or whether also the opposite is possible.

One study by Hutchison et al. (2019) used a longitudinal study to test children's numerical abilities in Kindergarten during the fall and spring. They took numerical range (subitizing and non-subitizing range) into account and the childrens' baseline symbolic and non-symbolic knowledge using number comparison tasks. Hutchison et al. (2019) concluded that a bidirectional relationship existed between the symbolic and non-symbolic performance in the subitizing range. However, in the larger non-subitizing range, only a unidirectional relationship was present; symbolic improvements resulted in non-symbolic improvements but not the other way around. They concluded that previous studies were missing information by not differentiating between the subitizing and non-subitizing ranges. The bidirectional association between non-symbolic and symbolic performance might reflect the direct mapping that only occurs in the subitizing range, similar to the results found in Chapter 2. In contrast, the non-subitizing range is speculated to rely on symbol-to-symbol associations (as proposed by Carey and Barner, 2019), likely leading to a better understanding of numerical relationships and relative quantities that affect non-symbolic acuity (as suggested by Matthews and Chesney, 2011). These results suggest different developmental trajectories for symbolic and non-symbolic abilities in the subitizing and non-subitizing ranges, making the distinction of these ranges crucial for numerical development research.

The study described in Chapter 3 took a developmental approach by adopting a mixed longitudinal (children 5/6-year-old, measured at kindergarten age and one year later in grade 1) and cross-sectional (a separate cohort of adults) design to test the ANS mapping account (Dehaene, 1996) and Exact Algorithm Hypothesis (Carey, 2009; Carey and Barner, 2019). We collected behavioral (response times and accuracy) and ERP data 
from the children and adults while they performed symbolic (Arabic digits) and non-symbolic (dot arrays) number comparison tasks. As explained in the General Introduction, number comparison tasks require participants to decide which of two numbers is larger. The performance depends on the numerical distance between two to-be-compared numbers. Numbers that are closer together (such as 4 and 5) are more difficult to compare/discriminate than two numbers that are further apart (such as 5 and 9). The overlapping (spatial) representations of the symbolic or non-symbolic numbers result in distance effects; a more accurate/faster magnitude comparison as numbers are numerically further apart. The presence of these distance effects is historically seen as a sign of recruitment of non-symbolic, analog (spatial) representations of magnitude.

Based on the aforementioned exact mapping hypothesis (Carey, 2009; Carey and Barner, 2019) we hypothesized that exact, one-to-one Arabic digit - quantity mapping would have only occurred between symbols and quantities in the subitizing range for children. Thus, the recruitment of analog (spatial) representations should only become visible as similar numerical distance effects across development for digits/quantities 1-4. In the non-subitizing range (6-9) the developmental number processing literature suggests that children rely more on other information like the ordinality of number sequences and symbol-symbol associations (Carey, 2009; Carey and Barner, 2019; Marinova and Reynvoet, 2020), resulting in weaker or absent symbolic distance effects. The results in Chapter 3 do indeed demonstrate typical number distance effects on reaction time and accuracy measures for Arabic digits and dot arrays in both the subitizing (1-4) and non-subitizing (6-9) ranges for children (at kindergarten and grade 1 age) and young adults. However, neural distance effects on P2p amplitude assumed to be associated with first access to magnitude information, did show developmental differences. Based on the Exact Mapping Hypothesis (Carey, 2009; Carey and Barner, 2019), we already hypothesized that the neural P2p distance effect was only present for the subitizing range in the symbolic and non-symbolic tasks for children. In addition, adults displayed P2p distance effects for both numerical ranges in both tasks. These results show diverging developmental patterns for the symbolic subitizing and non-subitizing ranges. Leading to the conclusion that only Arabic digits 1-4 gain meaning by mapping onto their corresponding non-symbolic representations of magnitude in the ANS, whereas Arabic digits 6-9 require other processes than direct non-symbolic mapping. 
The above-discussed results are also partly in line with the Discrete Semantic System (DSS) model proposed by Krajsci et al. (2017; 2016). The DSS suggests that the source of numerical knowledge is a semantic association network, similar to a lexicon. Quantities are associated with exact words like "one" or "two," but also with more general descriptions such as "small", "smaller", or in the case of a number comparison task with a set referent like the experiment in Chapter 3: "Smaller than five." Considering the results of the 5year-old children in our study within this theory, it is possible that the children developed exact, one-to-one, digit-magnitude representations for Arabic digits 1-4 but only had access to general, ordinal-based representations of digits representing magnitudes outside the subitizing range. These general representations would be in the form of the semantic concepts suggested by Krajsci et al. (2017; 2016), such as 'higher than five.' These general concepts would suffice in this comparison task where participants had to decide whether a presented symbol was smaller or larger than five. Such a strategy would result in behavioral distance effects due to the semantic associations but would lack the numerical (quantity) associations that lead to neural P2p distance effects. Similar behavioral distance effects based on semantic associations, rather than numerical distance, have previously been found by Vigliocco et al. (2002) and Chiao et al. (2004). Vigliocco et al. (2002) presented participants with a stream of images that were semantically close together (such as "finger" and "thumb") or further apart (such as "car" and "helicopter"). Participants needed to name these images as fast as possible, which resulted in behavioral distance effects that varied with the semantic distance between the current image and the previous. Chiao et al. (2004) showed similar distance effects in a comparison task where participants compared academic and military ranks; concepts with ordinality but no numerical value. Both studies conclude that non-numeric semantic associations can elicit behavioral distance effects. Consequently, distance effects in numerical comparison tasks cannot be used to determine whether numerical information is being accessed reliably. The addition of neural measures such as the P2p can prove crucial in making this distinction, as demonstrated in the current dissertation.

In the non-symbolic comparison task, similar to in the symbolic task, distance effects on reaction time were present for children and adults in both the non-symbolic subitizing and non-subitizing ranges. The children also showed a general increase in response time between Kindergarten and Grade 1 and a decrease in the number of errors made in the non-symbolic subitizing range, implying they took more time to process the non-symbolic arrays and became more accurate in determining the quantity. Children also showed similar distance effects on P2p amplitude in both tasks. These effects were only present for the 
subitizing range and did not show any developmental change between kindergarten to a year later in grade 1. Adults also showed similar distance effects on P2p amplitude in both tasks present for both numerical ranges. These non-symbolic effects are in line with studies reporting higher attention involved in subitizing (served by the OTS; Feigenson et al., 2004) than estimation processes (Anobile, Cicchini, et al., 2012; Anobile, Turi, et al., 2012; Burr et al., 2010). The latter used for enumeration of higher quantities when counting is not possible, as is the case with the short stimulus presentation times used in the current task. These results imply that the representational overlap thought to be responsible for the non-symbolic distance effects (see Introduction) is still developing in children and remains larger in the adult's subitizing range.

Another possibility might be that the larger distance effects for dot arrays 1-4 are primarily caused by relatively larger difficulties discriminating dot array four from the referent. The quantity of four may be exceeding the subitizing range, marking the transition between subitizing and estimation processes. A similar result, with ' 4 ' being more difficult, was also found in Chapter 2, and previous studies in 3-4-year-old preschoolers finding evidence for faster and easier non-symbolic - symbolic mapping for number symbols (words/digits) 1-3 (Benoit et al., 2013; Hurst et al., 2016; Lira et al., 2017; Odic et al., 2015).

\subsubsection{Conclusions about number symbol grounding and suggestions for future research}

The studies described in chapters 2 and $\mathbf{3}$ suggest a more complicated development of numeric cognition than previously assumed by the ANS mapping account or the Exact Algorithms Hypothesis. Numerical development is a complex topic, and the literature shows mixed results regarding how the symbolic and non-symbolic number systems affect each other. For example, several training studies conclude that practice with non-symbolic magnitudes also improves symbolic math performance (Hyde et al., 2014; Park and Brannon, 2013, 2014), suggesting that the Symbolic Number System (SNS) is affected by the non-symbolic ANS. However, these studies are often cross-sectional, leaving the direction of influence ambiguous. The few longitudinal studies that conclude a unidirectional developmental path from non-symbolic acuity to symbolic numeracy understanding (Mazzocco et al., 2011; Wang et al., 2016) did not control for the individual symbolic performance at baseline so that a definitive conclusion about the causal relationship or about symbolic improvement over time cannot be drawn. The longitudinal 
studies with children that did take into account the symbolic performance at the first measurement reported opposite effects with symbolic number performance predicting performance on non-symbolic magnitude comparison tasks, but not the other way around (Lyons et al., 2018; Matejko and Ansari, 2016; Mussolin et al., 2014). In an attempt to resolve these mixed results, Hutchison et al. (2019) investigated the mapping direction separately for the subitizing and non-subitizing ranges. They concluded that the direction of influence between the symbolic and non-symbolic system differs between the subitizing and non-subitizing ranges, with a bidirectional association in the former and a symbolic to non-symbolic association in the latter. The mixed results from previous studies were likely due to the difference between the subitizing and non-subitizing ranges confounding the results. Based on the literature discussed above and the studies presented in Chapters 2 and 3 , it seems that learning number symbols requires the combination of different aspects of various number representations, such as the quantity information in non-symbolic representations, the ordered nature of number words, and the spatial organization of numerals. However, the current dissertation cannot answer how larger symbols gain their meaning or how the relationship between the non-symbolic and symbolic number systems changes from childhood to adulthood. A more comprehensive longitudinal study is required to answer these questions. The data presented in the first two chapters do suggest that it is likely that those larger symbols are not mapped directly onto non-symbolic representations, and the literature proposes that these symbols gain meaning through algorithms such as counting.

In an expanded model, different numerical representations are transformed backand-forth until eventually all modalities are integrated into an interconnected numerical model (Lira et al., 2017). The mapping between the three representations (words, nonsymbolic quantities, and symbols) does not only rely on approximations of non-symbolic representations as previously assumed (Dehaene, 2001; Piazza, 2010), nor does it only rely on the generalization of principles learned by mapping symbols onto the subitizing range (Carey and Barner, 2019). Instead, the acquisition of number symbols seems to rely on the properties of multiple modalities to arrive at a coherent and interconnected model. Such a developmental pathway means that children will rely on different information and strategies depending on their developmental stage, and results might differ. Future studies could expand on these findings, preferably using longitudinal cross-modal mapping designs to determine the direction of symbolic or non-symbolic magnitude mapping (or lack thereof) in the subitizing and non-subitizing range as children grow from pre-knower to Cardinal Principles-knower stages and beyond. 
The current dissertation is limited in the conclusions it can draw, as the study described in Chapter 2 used adults, and as the children in the study described in Chapter 3 were 5-6 years old, well within the CP-knower stage. To better understand numerical development, and specifically numerical symbol development, it is important to capture the changes that occur before even the first symbols gain meaning. The current dissertation does show the added benefit of neural measures when investigating numerical cognition. Previous studies, both longitudinal and cross-sectional, were largely behavioral in design. However, behavioral distance effects (e.g., on reaction time) can be the result of non-numeric properties of the stimuli such as visual cues or inter-symbol associations (such as a verbal number word list or spatial ordering; Chiao et al., 2004; Krajcsi and Kojouharova, 2017; Krajcsi et al., 2016). We can determine which processes underlie these comparisons by studying the numerical distance effects on reaction times and the P2p component in a symbolic and non-symbolic number comparison task. Chapters 2 and $\mathbf{3}$ show how neural measures such as the $\mathrm{N} 1$ and $\mathrm{P} 2 \mathrm{p}$ can aid in interpreting such processes and, in some cases, give insight into whether there are shared representations or if numerical processes are even being used at all.

\subsection{Embodiment of numerical processing: evidence for internalized finger-based number representations}

As mentioned above, a second main aim addressed in this dissertation was to investigate whether we could find evidence for internalization of culture-specific finger-number postures in the adult brain and whether this leads to facilitated processing of other number symbols, such as Arabic digits. The previous paragraphs discussed an expanded numerical model that involves transformations between different non-symbolic and symbolic (words, digits) number representations that likely occur when children are still developing the symbolic number system. Over time these representations come together into an interconnected numerical model (Lira et al., 2017). Some authors have suggested that finger-number representations might be a fourth numeric code available to children and adults to represent numerical information (Di Luca and Mauro, 2011; Kreilinger et al., 2020).

One of the first methods children use to represent numerical principles is through finger use; more raised fingers equals a larger number (Butterworth, 1999; Fuson, 1988). The use of fingers to represent quantities allows children to experience different numerical 
principles such as ordinality (sequence of finger counting), cardinality (set size represented by the number of fingers raised), and one-to-one correspondence (Di Luca and Mauro, 2011; Fayol et al., 1998; Noël et al., 2005). Cardinality refers to the general concept of quantity, applicable to discrete sets and continuous measures. On the other hand, Ordinality refers to a relative position within a counting sequence (for a more in-depth discussion on these concepts, see Delazer and Butterworth, 1997; Fuson, 1988; Sury and Rubinsten, 2012). Studies with children indicate that ordinality and cardinality are two different mechanisms that seem to develop successively. Bermejo (1996) asked young children (3-4 years old) to count, and approximately $90 \%$ did so successfully. However, when asked how many there were (the cardinality of the set), only $45 \%$ were able to answer the question correctly. Young children seemingly possess ordinality (counting) without grasping the cardinality of the numbers they are reciting. Turconi, Jemel, Rossion \& Seron (2004) showed that this difference between ordinality and cardinality is also present at a neural level. In their study, participants performed comparison and ordinality tasks using number and letter stimuli. Behavioral results were similar for these tasks, showing the expected distance and SNARC effects regardless of the stimuli. However, electrophysical results showed different spatiotemporal activation patterns for cardinality and ordinality in the parietal and prefrontal areas, respectively.

Principles of ordinality and cardinality have rarely been investigated for finger counting, despite fingers having specific (canonical) finger postures to indicate numbers. Canonical finger-number representations are culturally acquired patterns taught by peers or parents. To show "two," one culture might raise their index and middle finger; any other non-canonical configuration would feel "strange." Such raising of fingers to show a quantity is also known as montring, and these can (but not always) differ from counting, which often involves the thumb and sequential raising of fingers (Di Luca and Pesenti, 2008; Pika et al., 2009). When children start to count on their fingers in early stages, specific fingers might have associations with a specific number. These associations between finger patterns and their numeric meaning can still be found in adults (Di Luca et al., 2006) and can affect how we process numbers in simple mathematics. The latter is shown by studies from Domahs et al. (2008) and Klein et al. (2011). They argue that finger-number representations in western cultures have a natural sub-base of five: ten fingers divided into two times five fingers. Klein et al. (2011) found that there was a significant response time increase in adults with addition problems that crossed a five boundary (e.g., $42+4$ crossing the 45 boundary, as opposed to $46+2$ staying within the 45-50 range). The response delay was thought to be rooted in finger-counting, where there 
is a motoric break when switching from one hand to another to continue counting (Klein et al., 2011). Domahs et al. (Domahs et al., 2008) concluded that the sub-base five number system also causes children to think in "fives" or "full hands". The quantity of seven is not represented by seven but as $2+5$; two fingers and one full hand. Likewise, the quantity of 12 is represented as $2+5+5$; two fingers and two full hands. There is a certain amount of "full-hands" combined with the one-handed representation of the remainder, similar to counting on one's hands. In their study, Domahs et al. (2008) found that when children performed addition and subtraction exercises, they made significantly more errors that deviated by exactly five (also known as split-five errors), a failure to represent a full hand and only focusing on the remainder. Even though the tasks in the aforementioned studies only used number symbols, the effects of early hand usage were still present in children and adults. Culturally learned finger-number patterns thus seem to play an important role in symbolic number development.

\subsubsection{The Facilitation Effect of Canonical Finger-Montring Patterns}

The studies described in Chapters 4 and 5 investigated the semantic processing of canonical versus non-canonical finger configurations in the adult brain and their possible facilitatory effects on the retrieval and processing of the numeric meaning of other standard number symbols (e.g., Arabic digits). The prior behavioral literature on finger-number representations concludes that, through repeated use, canonical patterns gained stronger semantic associations with their numerosities. These associations were assumed to be stored in long-term memory and result in faster retrieval of the pattern's meaning (Badets et al., 2010; Di Luca et al., 2006; Di Luca and Mauro, 2011; Di Luca and Pesenti, 2008). Non-canonical patterns have no such associations and are not stored in memory, and thus require counting strategies to determine their exact numeric meaning. The study described in Chapter $\mathbf{4}$ used a math verification paradigm in which participants verified a math-problem solution shown as canonical or non-canonical finger-number representations. The follow-up study described in Chapter $\mathbf{5}$ used a same-different task wherein participants saw an Arabic numeral flanked by canonical or non-canonical representations.

The behavioral data in chapters $\mathbf{4}$ and $\mathbf{5}$ replicate the earlier behavioral findings from prior studies by showing faster magnitude processing/comparison of canonical compared to non-canonical finger-number configurations, in two different tasks and two different samples of young adults. The current studies added to the literature by including ERP 
measures to support further the hypothesis that only canonical finger-number patterns have gained semantic associations in long-term memory. The use of ERP measures allows for the investigation of specific processes, allowing us to confirm or reject existing hypotheses concerning numerical processing. In the case of chapters 4 and 5, the ERP measures showed that canonical, compared to non-canonical, finger-number patterns elicited higher amplitude of the P2p ERP component. We interpreted this P2p increase as an early crossmodal semantic process, where a visual pattern is integrated with its (numerical) meaning (Lockwood and Tuomainen, 2015). In the case of finger-number patterns, the visual shape of a canonical pattern elicits a stronger response to a semantic number meaning than a noncanonical pattern, which supports previous suggestions based on behavioral data (Di Luca and Mauro, 2011). The second component we measured was the P3, which, similarly to the P2p, showed higher amplitudes to canonical compared to non-canonical finger-number patterns. We interpreted the enhanced P3 response as a late semantic process, closer to the response decision, reflecting memory recognition and increased response confidence (Eimer and Mazza, 2005; Friedman and Johnson, 2000; Polich, 2003; Soylu et al., 2019; Ye et al., 2019). Based on these findings, we conclude that, due to their stronger semantic associations, the canonical finger-number patterns were easier to categorize and classify, increasing the subjective confidence one had in deciding whether the hands matched a solution kept in memory (Chapter 4) or a symbol shown at the same time (Chapter 5). These processes were previously hypothesized based on behavioral results, which have now been shown using neural measures. In addition, the P2p and P3 ERP components have also been shown to be fitting indices for the numerical processing of finger-number representations, which may be used in future research.

\subsubsection{Differences between the Subitizing and Non-Subitizing Range}

As mentioned in previous sections, numerical development is a complex topic. The differentiation between the subitizing and non-subitizing ranges is important to understand numerical development from pre-symbolic to an adult symbolic number system. For this reason, the finger-number gesture studies in chapters $\mathbf{4}$ and $\mathbf{5}$ also distinguished between finger representations of numbers in the subitizing (1-4) and non-subitizing (6-9) ranges.

The behavioral results in Chapter 4, using the math verification task, showed a three-way interaction between canonicity, range, and solution. The results indicate that if the finger pattern showed the expected solution, a facilitation effect (faster responses to canonical representations) was present in the subitizing and non-subitizing ranges. 
However, if the solution did not match the expected results, facilitation was only present in the subitizing range. In the Chapter 5 follow-up study using the same-different task, we did not find the same three-way interaction in the behavioral data. Facilitation (a canonicity effect) was only present in the subitizing range and only for trials in which the Arabic numeral and hands showed the same (congruent) number.

A common behavioral result is the aforementioned facilitative effect of canonical finger-number representations on numerical processing. The facilitative effect is present in the subitizing range for both studies described in Chapters 4 and 5. However, there are notable differences between the two studies in terms of these facilitation effects. The results discussed in Chapter 4 showed facilitation effects in the subitizing and non-subitizing ranges, whereas the experiment described in Chapter $\mathbf{5}$ only found these facilitation effects in the subitizng range. Chapter 5 also found that the math verification task responses were faster in the subitizing range for incorrect solution (incongruent) trials. In contrast, in the same/different task used in Chapter 4, facilitation was only present on congruent trials. At current there is no clear explanation for these differences; they might be due to differences between the two tasks. In the math task, one first computed a sum which was held in memory after which hands were presented, and matching was required, whereas, in the same-different comparison task, hands and Arabic digits were presented simultaneously within one stimulus display. Future studies are needed to investigate how the facilitative effect is affected by these differences.

As end-point measures, behavioral results only provide a partial picture of the processes that underlie the processing of finger-number representations. The current dissertations added neural measures to investigate the underlying processes that lead to these behavioral results. In the math verification task (Chapter 4), a higher P2p amplitude in the subitizing range compared to the non-subitizing range was found. This difference between ranges was also larger if the solution was correct, i.e., matched the expected value held in memory. The difference between the smaller and larger range was also present in the second (but not the first) P2p window of the same-different task used in Chapter 5. However, unlike Chapter 4, the range effect did not differ between congruent and incongruent trials. We assumed that the P2p represents an early cross-modal semantic process, where a visual pattern is integrated with its (numerical) meaning (Lockwood and Tuomainen, 2015). Thus, the current results imply that finger-number patterns in the subitizing range that show a solution congruent with the expected solution recruit more cognitive resources. Congruent solutions in the non-subitizing range seemingly do not 
integrate the finger-number patterns and the expected solution. They therefore do not recruit additional cognitive resources as was the case in the subitizing range. Such an interpretation is in line with the response times being faster in the subitizing range; the recruitment of more cognitive resources leads to faster recognition of the numerical meaning of the hands. In Chapter 4, the range difference in P2p amplitude was also larger if the finger-number patterns showed a numerosity that matched the previously computed correct answer to the math equation (compared to one that did not match). The integration process for these finger-number patterns was likely easier since the associated numerosity was already activated before the hand stimuli were presented. On incorrect solution trials, the numerosity shown by the finger pattern still needed to be retrieved before integration could occur. This was not the case in Chapter 5, where the numeral and finger-number patterns were shown simultaneously. The simultaneous presentation precluded the activation of a numerosity before the stimulus was shown, resulting in similar processes for congruent and incongruent trials. However, more research is needed to determine if these differences in stimulus presentation were responsible for the differences in $\mathrm{P} 2 \mathrm{p}$ findings.

The second ERP component under investigation in Chapters 4 and 5 was the P3 component. Chapter 4 showed a larger P3 response for correct solutions compared to incorrect solutions in both ranges, but this difference was larger in the subitizing range. Chapter 5 showed a significantly higher P3 response for the subitizing compared to the non-subitizing range, and this range difference was present in both congruent and incongruent trials. In line with the previous ERP literature, we interpret the higher P3 response to canonical finger patterns presenting a number in the subitizing range as reflecting easier categorization of the stimuli, resulting in higher response confidence (Eimer and Mazza, 2005; Friedman and Johnson, 2000; Polich, 2003; Soylu et al., 2019; Ye et al., 2019). One possible explanation for the difference in P3 results in these chapters is similar to that of the P2p component. The research design differed in the type of comparison (mental representation versus an on-screen numeral) and how the stimuli were presented (sequentially versus simultaneously). The current experiments cannot conclusively determine how these differences might have affected the P3 results. 


\subsubsection{Hemispheric differences: Finger-Montring Patterns as Ideographs}

An overlapping finding in both studies was that the P2p and P3 canonicity effects were stronger above the right-hemispheric occipital-parietal cortex. These results are similar to those reported in previous studies investigating the neural correlations of cross-modal mapping of ideographs. Ideographs are visual symbols that represent an idea or concept, independent of language. Such concepts can be the "peace" or "ok" sign using one's hands, an emoji, or a numerical symbol such as roman numerals (II or IV) or Chinese ideographs (五 or 六 ). Two ERP studies (Bien et al., 2012, 2013; Lockwood and Tuomainen, 2015) found similar increases in P2 amplitude as a response to ideographs compared to arbitrary non-ideographic controls. The authors interpreted the increase in P2 amplitude as reflecting the sensory integration processes between an ideograph and the information triggered by that ideograph in another modality. However, these studies did not include numerical ideographs as in the current finger-number studies. Numerical ideographs were used in an fMRI study by Holloway et al. (2013), who compared Arabic numerals to Chinese number ideographs in bilingual English-Chinese participants and bilingual participants with no knowledge of Chinese ideographs. In both groups, the Arabic numerals (e.g., 2 or 3 ) elicited activations in the left intra-parietal sulcus (IPS), which was expected. However, the Chinese number ideographs (e.g., 五 or 六) only activated the right IPS in Chinese speakers familiar with the cultural meaning of these ideographs. We speculate that culturally learned canonical finger-montring patterns in the current experiments might have attained a similar ideographic status, resulting in the rightlateralized activity measured in Chapters 4 and 5. Unfortunately, the current studies did not compare hemispheric differences between different forms of numerical representations. The conclusions in the current dissertation about finger-number representations and their status as symbolic or ideographic remain speculative. Ideally, they should be replicated using cross-modal comparison tasks using finger representations, number words, Arabic numerals, and ideographs. 


\subsubsection{Concluding remarks and Future Directions}

In summary, the studies in Chapters $\mathbf{4}$ and $\mathbf{5}$ show that canonical finger numbers can facilitate number processing, and this facilitation is stronger in the subitizing range. However, the behavioral and neural results show some mixed results in the non-subitizing range and the incorrect/incongruent conditions, which might be caused by differences between the used tasks in the different studies. Nonetheless, the results indicate differences between the subitizing and non-subitizing ranges, possibly reflecting the use of hands in numerical development. As mentioned in previous sections, children likely transform between various numerical modalities or representations for different numerical tasks before these modalities are integrated into a cohesive numerical model (Lira et al. 2017). One way finger-numbers could mediate such a back-and-forth transformation phase in early childhood would be to act as a familiar reference or a memory aid for numerical representations when memory capacity and number strategy use is still immature (Geary, 2004; Gelman and Gallistel, 1978; Soylu et al., 2018). Consequently, the associations between hands and their numerosities are stronger in the small range compared to the larger range, where numbers likely gain meaning through inference.

Another explanation for the behavioral and neural differences between the facilitating effect of finger-numbers in the subitizing and non-subitizing range could be the frequency at which small and large finger-number representations are used. In daily life, it is more common to use the smaller single-handed signs than the larger two-handed finger patterns. The smaller range is practiced and used more often, leading to stronger semantic associations between the numerosities and representations. The current dissertation cannot give a conclusive answer on the role of finger-number representations during numerical development because only adults were studied with well-developed number systems. However, the current study identifies mechanisms for processing finger-number representation and possible neural indices to study them. More research is needed to determine why finger-number representations have stronger associations with their numerical meaning in the subitizing range and why under certain conditions also in the non-subitizing range. These could be remnants of learning processes used during early childhood, which is still present in adults. Future studies should use these insights to investigate younger child populations who are still learning number symbols. The current findings provide a good basis for such research, highlighting the importance of distinguishing between the subitizing and non-subitizing ranges and the usefulness of ERP measures, especially the P2p and P3 components. 


\subsection{General Conclusion}

The studies presented in this dissertation investigated how numerical symbols (such as Arabic numerals and finger-number patterns) are represented and processed in the brain. The ANS mapping account proposed that all symbols are learned by mapping onto nonsymbolic quantities, but the current thesis's findings do not align with that assumption. The results in the present thesis are more in line with the Exact Algorithms Hypothesis (Carey and Barner, 2019) that states that one-to-one non-symbolic-symbolic mapping only occurs in the subitizing range, highlighting the importance of investigating the small and large number range separately. However, this hypothesis does not consider some of the developmental changes that occur during numerical development. In the subitizing range, the association between symbolic and non-symbolic representations seems to be bidirectional. The hypothesis mentioned that symbols gain meaning by mapping onto nonsymbolic quantities but does not include the later stage where the quality of non-symbolic quantities also improves through a deeper understanding of numbers brought on by the symbolic number system.

Similarly, the hypothesis states that the non-subitizing range gains numerical meaning through inference and algorithms that rely on symbol-to-symbol associations but does not include the unidirectional effects symbolic learning has on non-symbolic representations. An expanded view on numerical development is needed that integrates the recent insights (such as bi-directionality of learning) and the current results presented in this thesis. In this expanded model neural measures such as the N1, P2p, and P3 ERP components would prove useful for investigating the longitudinal development of aspects such as numerical access.

Additionally, we investigated the potential facilitatory effects of finger-number representations on the processing of other numbers, which would point to their internalization. As for the facilitating effects of canonical finger-number postures, we concluded that canonical finger-number representations likely constitute ideographic representations, allowing for faster and more accurate access to their meaning. Canonical finger-number representations facilitate numerical processing, and this effect is seemingly stronger in the subitizing range. These results might be a consequence of reliance on finger-number representations during early numerical development, where they mediated the one-to-one mapping between symbolic and non-symbolic representations in the subitizing range, but this needs to be further explored in longitudinal studies with younger populations. 
Numerical development is more complicated than the initial models proposed, and even newer models cannot yet account for all the observations, as mentioned previously. More research is needed to understand the interplay of different numerical representations and mapping directions between symbolic and non-symbolic representations at different numerical development stages. Such an expanded model would include the relationships between non-symbolic and symbolic number systems, a differentiation between the subitizing and non-subitizing range, and the inclusion of ideographic representations such as hands. 


\subsection{References}

Anobile, G., Cicchini, G. M., \& Burr, D. C. (2012). Linear mapping of numbers onto space requires attention. Cognition, 122(3), 454-459. https://doi.org/10.1016/j. cognition.2011.11.006 (cited on page 213)

Anobile, G., Turi, M., Cicchini, G. M., \& Burr, D. C. (2012). The effects of cross-sensory attentional demand on subitizing and on mapping number onto space. Vision Research, 74, 102-109. https://doi.org/10.1016/j.visres.2012.06.005 (cited on page 213)

Badets, A., Pesenti, M., \& Olivier, E. (2010). Response-effect compatibility of fingernumeral configurations in arithmetical context. Quarterly Journal of Experimental Psychology, 63(1), 16-22. https://doi.org/10.1080/17470210903134385 (cited on page 217)

Benoit, L., Lehalle, H., Molina, M., Tijus, C., \& Jouen, F. (2013). Young children's mapping between arrays, number words, and digits tt -. Cognition TA, 129(1), 95-101 (cited on pages 205, 213).

Bermejo, V. (1996). Cardinality development and counting. Developmental Psychology, 32(2), 263-268. https://doi.org/10.1037/0012-1649.32.2.263 (cited on page 216)

Bien, N., ten Oever, S., Goebel, R., \& Sack, A. T. (2012). The sound of size. crossmodal binding in pitch-size synesthesia: A combined tms, eeg and psychophysics study. NeuroImage, 59(1), 663-672. https://doi.org/10.1016/j.neuroimage.2011.06.095 (cited on page 221)

Bien, N., ten Oever, S., Goebel, R., \& Sack, A. T. (2013). Corrigendum to 'the sound of size' crossmodal binding in pitch-size synesthesia: A combined tms, eeg and psychophysics study. NeuroImage, 72, 325. https://doi.org/10.1016/j.neuroimage. 2012.12.006 (cited on page 221)

Burr, D. C., Turi, M., \& Anobile, G. (2010). Subitizing but not estimation of numerosity requires attentional resources. Journal of Vision, 10(6), 20-20. https://doi.org/10. $1167 / 10.6 .20$ (cited on page 213)

Butterworth, B. (1999). The mathematical brain (illustrate). Macmillan. (Cited on page 215$)$.

Carey, S. (2009). The origin of concepts (Volume 133). Oxford University Press. https: //doi.org/10.1093/acprof:oso/9780195367638.001.0001. (Cited on pages 204, 210, 211) 
Carey, S., \& Barner, D. (2019). Ontogenetic origins of human integer representations. Trends in Cognitive Sciences, 23(10), 823-835. https://doi.org/10.1016/j.tics. 2019.07.004 (cited on pages 205, 207, 210, 211, 214, 223)

Chiao, J. Y., Bordeaux, A. R., \& Ambady, N. (2004). Mental representations of social status. Cognition, 93(2). https://doi.org/10.1016/j.cognition.2003.07.008 (cited on pages 212,215 )

Dehaene, S. (1996). The organization of brain activations in number comparison: Event-related potentials and the additive-factors method. Journal of Cognitive Neuroscience, 8(1), 47-68. https://doi.org/10.1162/jocn.1996.8.1.47 (cited on pages 204,210$)$

Dehaene, S. (2001, February). Précis of the number sense. Mind \& language (Pages 16-36). https://doi.org/10.1111/1468-0017.00154. (Cited on pages 204, 205, 214)

Dehaene, S. (2011). The number sense. how the mind creates mathematics, revised and updated edition. Oxford University Press. https://doi.org/10.2307/2589308. (Cited on page 205)

Delazer, M., \& Butterworth, B. (1997). A dissociation of number meanings. Cognitive Neuropsychology, 14(4), 613-636. https://doi.org/10.1080/026432997381501 (cited on page 216)

Di Luca, S., Granà, A., Pesenti, M., Semenza, C., \& Seron, X. (2006). Finger-digit compatibility in arabic numeral processing. Quarterly Journal of Experimental Psychology, 59(9), 1648-1663. https://doi.org/10.1080/17470210500256839 (cited on pages 216, 217)

Di Luca, S., \& Mauro, P. (2011). Finger numeral representations: More than just another symbolic code. Frontiers in Psychology, 2(NOV), 272. https://doi.org/10.3389/ fpsyg.2011.00272 (cited on pages 215-218)

Di Luca, S., \& Pesenti, M. (2008). Masked priming effect with canonical finger numeral configurations. Experimental Brain Research, 185(1), 27-39. https://doi.org/10. 1007/s00221-007-1132-8 (cited on pages 216, 217)

Domahs, F., Krinzinger, H., \& Willmes, K. (2008). Mind the gap between both hands: Evidence for internal finger-based number representations in children's mental calculation. Cortex, 44(4), 359-367. https://doi.org/10.1016/j.cortex.2007.08.001 (cited on pages 216,217 )

Donchin, E. (1981). Surprise!...surprise? Psychophysiology, 18(5), 493-513. https://doi. org/10.1111/j.1469-8986.1981.tb01815.x (cited on page 204) 
Duncan, G. J., Dowsett, C. J., Claessens, A., Magnuson, K., Huston, A. C., Klebanov, P., Pagani, L. S., Feinstein, L., Engel, M., Brooks-Gunn, J., Sexton, H., Duckworth, K., \& Japel, C. (2007). School readiness and later achievement. Developmental Psychology, 43(6), 1428-1446. https://doi.org/10.1037/0012-1649.43.6.1428 (cited on page 204)

Eimer, M., \& Mazza, V. (2005). Electrophysiological correlates of change detection. Psychophysiology, 42(3), 328-342. https://doi.org/10.1111/j.1469-8986.2005. 00285.x (cited on pages 218, 220)

Fayol, M., Barrouillet, P., \& Marinthe, C. (1998). Predicting arithmetical achievement from neuropsychological performance: A longitudinal study. Cognition, 68(2), B63-B70. https://doi.org/10.1016/S0010-0277(98)00046-8 (cited on page 216)

Feigenson, L., Dehaene, S., \& Spelke, E. (2004). Core systems of number. Trends in Cognitive Sciences, 8(7), 307-314. https://doi.org/10.1016/j.tics.2004.05.002 (cited on page 213)

Friedman, D., \& Johnson, R. (2000). Event-related potential (erp) studies of memory encoding and retrieval: A selective review. Microscopy Research and Technique, 51(1), 6-28 (cited on pages 204, 218, 220).

Fuson, K. C. (1988). Children's counting and concepts of number (Illustrate, Volume 31). Springer New York. https://doi.org/10.1007/978-1-4612-3754-9. (Cited on pages 215,216 )

Geary, D. C. (2004). Mathematics and learning disabilities. Journal of Learning Disabilities, 37(1), 4-15. https://doi.org/10.1177/00222194040370010201 (cited on page 222)

Gelman, R., \& Gallistel, C. R. (1978). The child's understanding of number. Harvard University Press. (Cited on page 222).

Göbel, S. M., Watson, S. E., Lervåg, A., \& Hulme, C. (2014). Children's Arithmetic Development: It Is Number Knowledge, Not the Approximate Number Sense, That Counts. Psychological Science, 25(3), 789-798. https://doi.org/10.1177/ 0956797613516471 (cited on page 204)

Hersh, R., Dehaene, S., \& Hersh, R. (1999). The number sense: How the mind creates mathematics. The American Mathematical Monthly, 105(10), 975. https://doi.org/ 10.2307/2589308 (cited on page 205)

Holloway, I. D., \& Ansari, D. (2009). Mapping numerical magnitudes onto symbols: The numerical distance effect and individual differences in children's mathematics 
achievement. Journal of Experimental Child Psychology, 103(1), 17-29. https: //doi.org/10.1016/j.jecp.2008.04.001 (cited on page 204)

Holloway, I. D., Battista, C., Vogel, S. E., \& Ansari, D. (2013). Semantic and perceptual processing of number symbols: Evidence from a cross-linguistic fmri adaptation study. Journal of cognitive neuroscience, 25(3), 388-400. https://doi.org/10.1162/ jocn_a_00323 (cited on page 221)

Hurst, M., Anderson, U., \& Cordes, S. (2016). Mapping among number words, numerals, and nonsymbolic quantities in preschoolers. Journal of Cognition and Development, 18(1), 41-62. https://doi.org/10.1080/15248372.2016.1228653 (cited on page 213)

Hutchison, J. E., Ansari, D., Zheng, S., De Jesus, S., \& Lyons, I. M. (2019). The relation between subitizable symbolic and non-symbolic number processing over the kindergarten school year. Developmental Science, 00:e12884. https://doi.org/10. 1111/desc.12884 (cited on pages 210, 214)

Hyde, D. C., Khanum, S., \& Spelke, E. S. (2014). Brief non-symbolic, approximate number practice enhances subsequent exact symbolic arithmetic in children. Cognition, 131(1), 92-107. https://doi.org/10.1016/j.cognition.2013.12.007 (cited on page 213)

Hyde, D. C., \& Spelke, E. S. (2012). Spatiotemporal dynamics of processing nonsymbolic number: An event-related potential source localization study. Human Brain Mapping, 33(9), 2189-2203. https://doi.org/10.1002/hbm.21352 (cited on page 204)

Klein, E., Moeller, K., Willmes, K., Nuerk, H. C., \& Domahs, F. (2011). The influence of implicit hand-based representations on mental arithmetic. Frontiers in Psychology, 2(SEP), 197. https://doi.org/10.3389/fpsyg.2011.00197 (cited on pages 216, 217)

Krajcsi, A., \& Kojouharova, P. (2017). Symbolic numerical distance effect does not reflect the difference between numbers. Frontiers in Psychology, 8(NOV), 2013. https://doi.org/10.3389/fpsyg.2017.02013 (cited on pages 212, 215)

Krajcsi, A., Lengyel, G., \& Kojouharova, P. (2016). The source of the symbolic numerical distance and size effects. Frontiers in Psychology, 7, 1795. https://doi.org/10. 3389/fpsyg.2016.01795 (cited on pages 212, 215)

Kreilinger, I. L., Roesch, S., Moeller, K., \& Pixner, S. (2020). Mastery of structured quantities like finger or dice patterns predict arithmetic performance. Cognitive Processing, 1, 3. https://doi.org/10.1007/s10339-020-00994-4 (cited on page 215) 
Le Corre, M., \& Carey, S. (2007). One, two, three, four, nothing more: An investigation of the conceptual sources of the verbal counting principles. Cognition, 105(2), 395-438. https://doi.org/10.1016/j.cognition.2006.10.005 (cited on page 204)

Leibovich, T., \& Ansari, D. (2016). The symbol-grounding problem in numerical cognition: A review of theory, evidence, and outstanding questions. Canadian Journal of Experimental Psychology, 70(1), 12-23. https://doi.org/10.1037/cep0000070 (cited on page 204)

Libertus, M. E., Woldorff, M. G., \& Brannon, E. M. (2007). Electrophysiological evidence for notation independence in numerical processing. (Volume 3). https://doi.org/ 10.1186/1744-9081-3-1. (Cited on page 204)

Lira, C. J., Carver, M., Douglas, H., \& LeFevre, J. A. (2017). The integration of symbolic and non-symbolic representations of exact quantity in preschool children. Cognition, 166, 382-397. https://doi.org/10.1016/j.cognition.2017.05.033 (cited on pages $213-215,222$ )

Lockwood, G., \& Tuomainen, J. (2015). Ideophones in japanese modulate the p2 and late positive complex responses. Frontiers in Psychology, 6, 933. https://doi.org/10. 3389/fpsyg.2015.00933 (cited on pages 218, 219, 221)

Lyons, I. M., Bugden, S., Zheng, S., De Jesus, S., \& Ansari, D. (2018). Symbolic number skills predict growth in nonsymbolic number skills in kindergarteners. Developmental Psychology, 54(3), 440-457. https://doi.org/10.1037/dev0000445 (cited on page 214)

Marinova, M., \& Reynvoet, B. (2020). Can you trust your number sense: Distinct processing of numbers and quantities in elementary school children. Journal of Numerical Cognition, 6(3), 304-321. https://doi.org/10.5964/jnc.v6i3.292 (cited on page 211)

Matejko, A. A., \& Ansari, D. (2016). Trajectories of symbolic and nonsymbolic magnitude processing in the first year of formal schooling. PLoS ONE, 11(3). https://doi.org/ 10.1371/journal.pone.0149863 (cited on page 214)

Matthews, P., \& Chesney, D. (2011). Straightening up: Number line estimates shift from $\log$ to linear with additional information. Proceedings of the 33rd ..., 1936-1941 (cited on page 210).

Mazzocco, M. M., Feigenson, L., \& Halberda, J. (2011). Preschoolers' precision of the approximate number system predicts later school mathematics performance. $P L o S$ ONE, 6(9), 23749. https://doi.org/10.1371/journal.pone.0023749 (cited on page 213) 
Mussolin, C., Nys, J., Content, A., \& Leybaert, J. (2014). Symbolic number abilities predict later approximate number system acuity in preschool children. PLoS ONE, 9(3). https://doi.org/10.1371/journal.pone.0091839 (cited on page 214)

Noël, M. P., Gracia-Bafalluy, M., \& Noël, M. P. (2005). Finger gnosia: A predictor of numerical abilities in children? Cortex, 11(5), 413-430. https://doi.org/10.1080/ 09297040590951550 (cited on page 216)

Odic, D., Le Corre, M., \& Halberda, J. (2015). Children's mappings between number words and the approximate number system. Cognition, 138, 102-121. https : //doi.org/10.1016/j.cognition.2015.01.008 (cited on pages 205, 213)

Park, J., \& Brannon, E. M. (2013). Training the approximate number system improves math proficiency. Psychological Science, 24(10), 2013-2019. https://doi.org/10. 1177/0956797613482944 (cited on page 213)

Park, J., \& Brannon, E. M. (2014). Improving arithmetic performance with number sense training: An investigation of underlying mechanism. Cognition, 133(1), 188-200. https://doi.org/10.1016/j.cognition.2014.06.011 (cited on page 213)

Piazza, M. (2010). Neurocognitive start-up tools for symbolic number representations. Trends in Cognitive Science, 14(12), 542-551. https://doi.org/10.1016/j.tics.2010. 09.008 (cited on pages 204, 214)

Pika, S., Nicoladis, E., \& Marentette, P. (2009). How to order a beer: Cultural differences in the use of conventional gestures for numbers. Journal of Cross-Cultural Psychology, 40(1), 70-80. https://doi.org/10.1177/0022022108326197 (cited on page 216)

Pinel, P., Dehaene, S., Rivière, D., \& LeBihan, D. (2001). Modulation of parietal activation by semantic distance in a number comparison task. NeuroImage, 14(5), 1013-1026. https://doi.org/10.1006/nimg.2001.0913 (cited on page 204)

Polich, J. (2003). Theoretical overview of p3a and p3b. Detection of change (Pages 83-98). Springer US. https://doi.org/10.1007/978-1-4615-0294-4_5. (Cited on pages 218, 220)

Reynvoet, B., \& Sasanguie, D. (2016). The symbol grounding problem revisited: A thorough evaluation of the ans mapping account and the proposal of an alternative account based on symbol-symbol associations. Frontiers in Psychology, 7(OCT), 1581. https://doi.org/10.3389/fpsyg.2016.01581 (cited on page 204) 
Siegler, R. S. (2016). Magnitude knowledge: The common core of numerical development. Developmental Science, 19(3), 341-361. https://doi.org/10.1111/desc.12395 (cited on page 204)

Siegler, R. S., \& Ramani, G. B. (2009). Playing linear number board games-but not circular ones-improves low-income preschoolers' numerical understanding. Journal of Educational Psychology, 101(3), 545-560. https://doi.org/10.1037/a0014239 (cited on page 204)

Soylu, F., Lester Jr, F. K., Newman, S. D., Norton, A., \& Nurnberger-Haag, J. (2018). You can count on your fingers: The role of fingers in early mathematical development. Journal of Numerical Cognition, 4(1), 2363-8761. https://doi.org/10.5964/jnc. v4i1.85 (cited on page 222)

Soylu, F., Rivera, B., Anchan, M., \& Shannon, N. (2019). Erp differences in processing canonical and noncanonical finger-numeral configurations. Neuroscience Letters, 705, 74-79. https://doi.org/10.1016/j.neulet.2019.04.032 (cited on pages 218, 220)

Spelke, E. S. (2017). Core knowledge, language, and number. Language Learning and Development, 13(2), 147-170. https://doi.org/10.1080/15475441.2016.1263572 (cited on page 204)

Sullivan, J., \& Barner, D. (2012). How are number words mapped to approximate magnitudes? Quarterly Journal of Experimental Psychology, 66(2), 389-402. https://doi.org/10.1080/17470218.2012.715655 (cited on page 204)

Sury, D., \& Rubinsten, O. (2012, January 1). Ordinal processing of numerical and nonnumerical information. Reading, writing, mathematics and the developing brain: Listening to many voices (Pages 209-232). Springer Netherlands. https://doi.org/ 10.1007/978-94-007-4086-0_13. (Cited on page 216)

Szucs, D., \& Soltész, F. (2007). Event-related potentials dissociate facilitation and interference effects in the numerical stroop paradigm. Neuropsychologia, 45(14), 3190-3202. https://doi.org/10.1016/j.neuropsychologia.2007.06.013 (cited on page 204)

Temple, E., \& Posner, M. I. (1998). Brain mechanisms of quantity are similar in 5-year-old children and adults. Proceedings of the National Academy of Sciences of the United States of America, 95(13), 7836-41. https://doi.org/10.1073/pnas.95.13. 7836 (cited on page 204)

Turconi, E., Jemel, B., Rossion, B., \& Seron, X. (2004). Electrophysiological evidence for differential processing of numerical quantity and order in humans. Cognitive 
Brain Research, 21(1), 22-38. https://doi.org/10.1016/j.cogbrainres.2004.05.003 (cited on pages 204, 216)

Vigliocco, G., Vinson, D. P., Damian, M. F., \& Levelt, W. (2002). Semantic distance effects on object and action naming. Cognition, 85(3). https://doi.org/10.1016/S00100277(02)00107-5 (cited on page 212)

Wang, J., Odic, D., Halberda, J., \& Feigenson, L. (2016). Changing the precision of preschoolers' approximate number system representations changes their symbolic math performance. Journal of Experimental Child Psychology, 147, 82-99. https: //doi.org/10.1016/j.jecp.2016.03.002 (cited on page 213)

Wynn, K. (1990). Children's understanding of counting. Cognition, 36(2), 155-193. https: //doi.org/10.1016/0010-0277(90)90003-3 (cited on page 205)

Wynn, K. (1992). Children's acquisition of the number words and the counting system. Cognitive Psychology, 24(2), 220-251. https://doi.org/10.1016/0010-0285(92) 90008-P (cited on page 205)

Ye, M., Lyu, Y., Sclodnick, B., \& Sun, H. J. (2019). The p3 reflects awareness and can be modulated by confidence. Frontiers in Neuroscience, 13(MAY), 510. https: //doi.org/10.3389/fnins.2019.00510 (cited on pages 218, 220) 


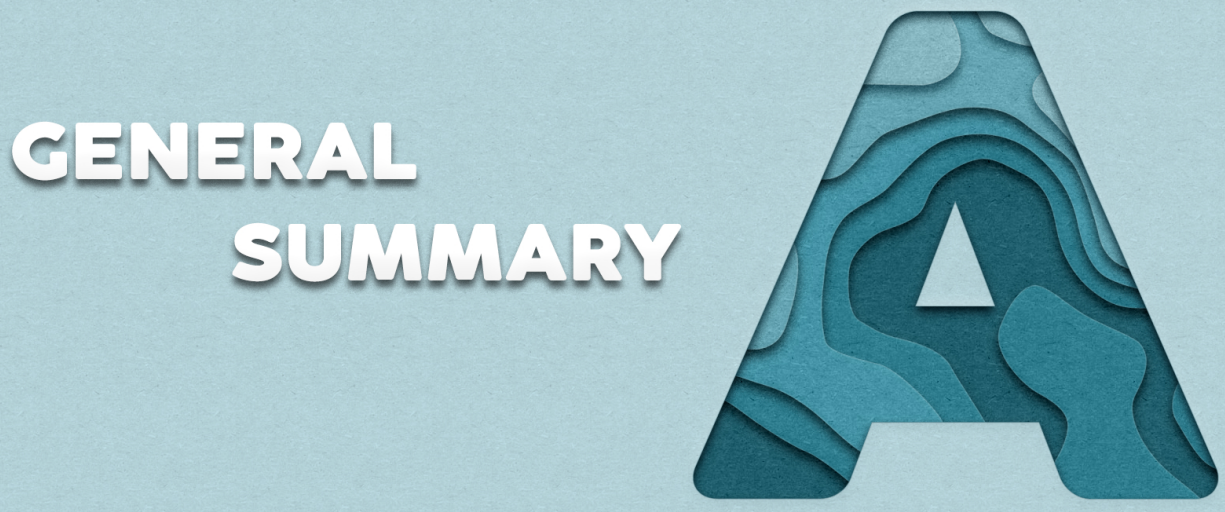

\section{General Summary}

Numerical symbols are an extremely important aspect of modern society. We need to use and understand numerals such as 3 and 7 to function in our daily lives, do our jobs, and manage our time and money. Humans learn the meaning of number symbols at a young age, but how these symbols gain meaning is still unclear. Early theories posit that symbols map onto an innate non-symbolic system (the Approximate Number System: ANS) representing analog quantities (such as the number of pens on a desk or birds in the skies). However, the main assumption of this ANS mapping account (Dehaene, 2001; Piazza, 2010), that number symbols (words/Arabic digits) directly map onto their non-symbolic counterparts in the ANS, has been questioned by the literature. An alternative account, the Exact Algorithm Hypothesis (Carey, 2009; Carey and Barner, 2019), is based on findings from the developmental number processing literature. This hypothesis posits that children can only establish direct associations between real-world quantities and numerical symbols or words in the smaller subitizing range (quantities 1 through 4 ). This account suggests that instead of mapping directly onto quantities, larger symbols ( 5 and above) gain meaning through symbol-to-symbol relationships (such as ordinality) and the principles these relationships give rise to (such as counting). There is increasing interest in this topic in recent years. However, studies investigating the possible differences in the acquisition/grounding (symbol - non-symbolic) of number symbols in or outside the subitizing range are still lacking. The four behavioral/electrophysiological studies in this thesis address the learning/representation of number symbols, especially differences in the subitizing range and beyond. 
The first two studies described in Chapters $\mathbf{2}$ and $\mathbf{3}$ tested the above-mentioned ANS/Exact mapping accounts, especially their application to number symbol learning in and beyond the subitizing range using different paradigms/designs in both studies. In both studies, we investigated the process of symbolic learning separately for the subitizing and non-subitizing range using behavioral and Event-Related Potential (ERP) measures. Chapter 2 describes an experimental study in young adults in which we tested the assumption of the ANS mapping account by mimicking the symbol learning process. The adults had to acquire the numeric meaning of novel number symbols by mapping them onto non-symbolic quantity information (presented as arrays of dots in the 1-9 range) in a trial-and-error learning task with feedback. The presentation time of the dot arrays was too short to allow the counting of items in the non-subitizing range, similar to how children need to learn without counting strategies. Behavioral results presented in this chapter indicated that only the novel symbols representing numbers 1-4 in the subitizing range gained an exact numerical meaning across learning blocks. The adults in this study successfully mapped symbols in the subitizing range onto non-symbolic quantities, but they could not do this for novel symbols representing larger numbers 6-9. The ERP measures (N1 and P2p) confirmed these behavioral results. The amplitude of the early N1 component in response to the novel symbols was affected by the numerosity of the non-symbolic arrays that preceded the new symbol. A larger negative amplitude was present when a non-matching symbol followed an array, but only for novel symbols in the subitizing range. We interpreted this N1 modulation as signifying a violation of expectation, which can only occur if participants extracted numerical meaning from the non-symbolic array and knew the matching symbol, as was the case for novel symbols 1-4. We included the later parietal P2p component, which occurs around 200 milliseconds post-stimulus in adults, based on prior number comparison studies. The $\mathrm{P} 2 \mathrm{p}$ is assumed to represent the processing stage in which one has first semantic access to the magnitude information represented by the symbol. The amplitude of the P2p is enhanced if more cognitive resources are needed to retrieve a symbol's numeric meaning. The P2p decreased in amplitude across learning blocks in the current learning study, but only for the first four symbols in the subitizing range and not for symbols representing a quantity outside the subitizing range. In line with the literature, this $\mathrm{P} 2 \mathrm{p}$ amplitude decrease for symbols 1-4, which we interpreted as less effort needed to retrieve the symbol's associated magnitude information with learning. 
In Chapter 3, we tested the ANS/Exact mapping accounts in a developmental study. In this study, 5/6-year-old children (measured twice: at kindergarten age and one year later in grade 1) and adults performed symbolic (Arabic digits) and non-symbolic (dot arrays) number comparison tasks while collecting accuracy/reaction time and ERP data. In such number comparison tasks, one has to decide which of two numbers is larger; performance depends on the numerical distance between two-to-be compared numbers. Numbers that are closer together (such as 4 and 5) are more difficult to compare/discriminate than two numbers that are further apart (such as 5 and 9). This difference is due to overlapping (spatial) representations resulting in the so-called distance effect marked by less accurate/slower magnitude comparison between numerically close than far numbers. The presence of distance effects in comparison tasks are historically interpreted as a sign of recruitment of non-symbolic, analog (spatial) representations of magnitude. In children (at kindergarten and grade 1 age) and young adults typical number distance effects were present on reaction time and accuracy measures for Arabic digits and dot arrays in both the subitizing (1-4) and non-subitizing (6-9) ranges. However, neural distance effects on P2p amplitude, which are assumed to be associated with an early processing stage in which one has first access to magnitude information, did show developmental differences. Whereas children only showed a P2p distance effect for the subitizing range in the symbolic and non-symbolic tasks, adults displayed P2p distance effects for both numerical ranges in both tasks. These combined results led to the conclusion that only Arabic digits 1-4 gain meaning by mapping onto their corresponding non-symbolic representations of magnitude in the ANS, whereas Arabic digits 6-9 require other processes than direct non-symbolic mapping. We speculate that the presence of a P2p distance effect for digits/quantities beyond the subitizing range in adults originates from further development of the symbolic number system. The studies in Chapters $\mathbf{2}$ and $\mathbf{3}$ thus provide evidence in favor of the Exact mapping hypothesis, with only number symbols 1-4 being learned by non-symbolic - symbolic mapping.

The second aim of this thesis was to investigate the semantic processing of culturespecific (canonical) finger-number representations and how these might facilitate adult symbolic number (Arabic digits) processing in the subitizing (1-4) and non-subitizing (6-9) ranges. The culture-typical pattern of raised fingers used to show a certain quantity is also known as a canonical finger-number pattern. The use of fingers to represent quantities is universal. Children display the ability to show a number on the fingers very early on in development, sometimes even before using the appropriate number words, pointing to a potentially important role of finger-number representations in early number symbol 
learning. The two ERP studies described in Chapters $\mathbf{4}$ and $\mathbf{5}$ used different task paradigms to investigate whether there are still signs of culture-specific finger-number representations influencing (facilitating) the processing of other learned number symbols (Arabic digits) in the young adult's brain. Such evidence would point to finger representations becoming embodied and signify the potential importance of fingers during early number symbol acquisition.

To investigate whether young adults displayed signs of embodied canonical finger-number representations, the study described in Chapter $\mathbf{4}$ used a math verification paradigm. We presented young adults with easy math problems followed by an answer in the form of a canonical or atypical (non-canonical) finger-number pattern. Participants verified the answer shown as canonical or non-canonical finger patterns against the currently activated solution in memory by indicating whether finger-pattern and solution showed the same or a different number. Participants' reaction time, accuracy, and parietal P2p amplitudes were measured and compared between match-canonical, match-non-canonical, non-match-canonical, and non-match non-canonical conditions. Chapter 5 investigated the same research question in another sample of young adults, now using a same/different paradigm. In this task, instead of comparing the number displayed by finger patterns to mentally represented sum solution, participants had to compare the number shown by an Arabic digit and finger pattern now displayed simultaneously within one stimulus display. The four conditions in this task were: canonical-congruent, canonical-incongruent, non-canonical-congruent and non-canonical-incongruent. In line with previous findings, we found that responses to canonical finger patterns were faster and more accurate. According to previous authors, such facilitating effects of canonical finger-number patterns on number processing are due to their storage and links with other symbolic number representations in long-term memory. These links allow for faster retrieval of a numerical meaning when seeing a canonical finger-number pattern. We investigated these claims using ERP measures in addition to behavioral measures, focusing on the P2p and P3 ERP components representing different stages of semantic number processing. In line with earlier studies using similar task paradigms, the occipital-parietal P2p indexes an early cross-modal semantic process. The centro-parietal $\mathrm{P} 3$ represents a late semantic process closer to the response decision, reflecting conscious memory recognition and increased response confidence. 
In both experiments, canonical finger patterns resulted in faster and more accurate responses and larger $\mathrm{P} 2 \mathrm{p}$ and $\mathrm{P} 3$ responses in the right hemisphere compared to non-canonical patterns. These neural measures confirm the hypotheses of earlier studies; canonical finger-number patterns seem to have become embodied and developed semantic associations with other number representations, allowing for faster retrieval of the numerical meaning and facilitating the processing of other numerals. In keeping with the studies in Chapters 2 and 3, these studies also investigated the facilitative effects of canonical finger-number patterns on Arabic digit processing separately for the subitizing (1-4) and non-subitizing (6-9) ranges. The results showed improved performance and stronger neural responses to canonical finger-number patterns in the subitizing range compared to higher numbers in both tasks. However, the study described in Chapter 4 also showed facilitation in the non-subitizing range when the solution matched the expectation and in the subitizing range when the solution did not match the expectation. These differences between the studies cannot be explained by the current data and needs further investigation. In addition, both studies showed right-hemispheric lateralization of the P2p above occipital-parietal electrode locations. Based on prior studies, we tentatively interpreted this lateralization as embodied canonical finger-number patterns being more similar to icons or ideographs, visual representations of an idea (such as an emoji), separate from language. These data suggest that canonical finger-number patterns might have an important role in early number acquisition, especially for small numbers in the subitizing range.

The studies presented in this dissertation provide more insight into the neural mechanisms underlying number symbol learning and the facilitative function of canonical finger number representation. These findings and the used methods/paradigms could aid the design of future studies investigating early number symbol learning within more extensive longitudinal designs. Eventually, the neural correlates of number/magnitude processing reported in our studies might also be useful to investigate the individual differences in number processing that might arise in early development but can have profound consequences in later years. With society becoming more digital and reliant on numerical information, the ability to process number symbols is quickly becoming more and more important. 


\section{References}

Carey, S. (2009). The origin of concepts (Volume 133). Oxford University Press. https: //doi.org/10.1093/acprof:oso/9780195367638.001.0001. (Cited on page 233)

Carey, S., \& Barner, D. (2019). Ontogenetic origins of human integer representations. Trends in Cognitive Sciences, 23(10), 823-835. https://doi.org/10.1016/j.tics. 2019.07.004 (cited on page 233)

Dehaene, S. (2001, February). Précis of the number sense. Mind \& language (Pages 16-36). https://doi.org/10.1111/1468-0017.00154. (Cited on page 233)

Piazza, M. (2010). Neurocognitive start-up tools for symbolic number representations. Trends in Cognitive Science, 14(12), 542-551. https://doi.org/10.1016/j.tics.2010. 09.008 (cited on page 233) 


\section{ALeEMUING SAUIENVAUTLNO}

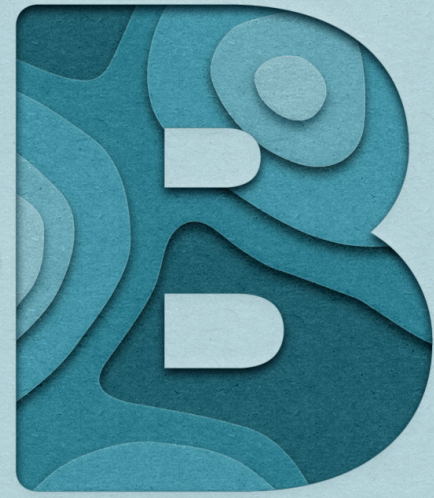

\section{Algemene Samenvatting}

Numerieke symbolen zijn een uiterst belangrijk aspect van de moderne samenleving. We moeten getallen zoals 3 en 7 kunnen begrijpen en gebruiken om in ons dagelijks leven te kunnen functioneren, ons werk te doen, en zelfs om tijd en geld te beheren. Mensen leren de betekenis van getalsymbolen al op jonge leeftijd, maar hoe deze symbolen betekenis krijgen is nog steeds onduidelijk. Vroege theorieën stelde voor dat symbolen aan een aangeboren non-symbolisch systeem gekoppeld werden (het Approximate Number System: ANS) dat analoge hoeveelheden weergeeft (zoals het aantal pennen op een bureau of het aantal vogels in de lucht). De belangrijkste aanname van deze ANS mapping account (Dehaene, 2001; Piazza, 2010), dat getalsymbolen (woorden/Arabische cijfers) direct gekoppeld worden aan niet-symbolische tegenhangers in de ANS, wordt echter in twijfel getrokken door de literatuur. Een alternatieve verklaring, de Exacte Algoritme Hypothese (Exact Algorithm Hypothesis; Carey, 2009; Carey and Barner, 2019), is gebaseerd op bevindingen uit de numerieke ontwikkelingsliteratuur. Deze hypothese stelt dat kinderen alleen directe associaties kunnen leggen tussen hoeveelheden in de echte wereld en numerieke symbolen of woorden in de kleinere subitizing range (hoeveelheden 1 tot en met 4). Deze verklaring suggereert dat grotere symbolen (5 en hoger) betekenis krijgen door symbool-tot-symbool relaties (zoals volgorde/ordinaliteit) en de principes waartoe deze relaties aanleiding geven (zoals tellen), in plaats van een directe koppeling met een niet-symbolische hoeveelheid. De laatste jaren is er toenemende belangstelling voor dit onderwerp. Echter, er is nog een gebrek aan studies die de mogelijke verschillen onderzoeken tussen het leren van numerieke symbolen binnen in buiten de subitizing range. De vier gedrags- en elektrofysiologische studies in dit proefschrift richten zich op het leren en representeren van getalsymbolen, in het bijzonder op verschillen tussen de subitizing range en hoger. 
De eerste twee studies beschreven in Hoofdstukken 2 en $\mathbf{3}$ testten de bovengenoemde ANS/Exact mapping verklaringen, in het bijzonder hun toepassing op het leren van getallensymbolen binnen en buiten de subitizing range, met behulp van verschillende paradigma's en test-ontwerpen. In beide studies onderzochten we het proces van symbolisch leren afzonderlijk voor de subitizing en non-subitizing range met behulp van gedragsmetingen en Event-Related Potential (ERP) metingen. Hoofdstuk 2 beschrijft een experimentele studie bij jongvolwassenen waarin we de aanname van het ANS mapping verklaring testten door het vroege symbolisch leerproces na te bootsen. De volwassenen moesten de numerieke betekenis van nieuwe getalsymbolen leren door deze te koppelen aan niet-symbolische kwantitatieve informatie (gepresenteerd als stip-patronen met 1-9 stippen) in een trial-and-error leertaak met feedback. De presentatie-tijd van de non-symbolische stip-patronen was te kort om deze te tellen, vergelijkbaar met hoe kinderen deze koppeling moeten maken zonder tel-strategieën. De gedragsresultaten gepresenteerd in dit hoofdstuk gaven aan dat alleen symbolen die stonden voor de getallen 1-4 een exacte numerieke betekenis kregen aan het einde van de leerperiode. De volwassenen in deze studie slaagden erin om symbolen in de subitizing range te koppelen aan niet-symbolische hoeveelheden, maar ze konden dit niet doen voor grotere symbolen (6-9). De ERP metingen (N1 en P2p) bevestigden deze gedragsdata. De amplitude van de vroege N1 component in reactie op de nieuwe symbolen werd beïnvloed door het aantal stippen dat voorafging aan het symbool. Een grotere negatieve amplitude was aanwezig wanneer het symbool niet overeenkwam met het aantal stippen, maar alleen voor symbolen in de subitizing range. We interpreteerden deze N1 modulatie als een schending van de verwachting (violation of expectation), die alleen kan voorkomen wanneer een deelnemer de exacte hoeveelheid stippen weet en de exacte betekenis kent van het bijbehorende symbool, zoals het geval was voor de nieuwe symbolen 1-4. De latere pariëtale P2p component, die bij volwassenen ongeveer 200 milliseconden na de stimulus optreedt, werd meegenomen, gebaseerd op eerdere studies naar getalvergelijking. De P2p wordt verondersteld de verwerkingsfase te vertegenwoordigen waarin men voor het eerst semantische toegang heeft tot de numerieke-informatie die door het symbool wordt gerepresenteerd. De amplitude van de P2p neemt toe als meer cognitieve middelen nodig zijn om de numerieke betekenis van een symbool op te halen. 
De P2p verminderde in amplitude over leerblokken in de huidige studie, maar alleen voor de eerste vier symbolen in de subitizing range en niet voor hogere symbolen. In lijn met de literatuur interpreteerde we deze verlaging van $\mathrm{P} 2 \mathrm{p}$ amplitude als een representatie van minder cognitieve moeite die nodig is om de numerieke betekenis van het getal op te halen wanneer deze geleerd worden.

In Hoofdstuk 3 hebben we de ANS/Exact mapping verklaringen getest in een ontwikkelingsstudie. In deze studie voerden 5/6-jarige kinderen (tweemaal gemeten: in groep 1 en groep 2) en volwassenen een symbolische (Arabische cijfers) en niet-symbolische (stip-patronen) nummer-vergelijkingstaken uit, terwijl nauwkeurigheids-/reactietijd- en ERP-gegevens verzameld werden. In dergelijke nummer-vergelijkingstaken moet men beslissen welk van twee getallen groter is; de prestatie hangt af van de numerieke afstand tussen de twee te-vergelijken getallen. Getallen die dichter bij elkaar liggen (zoals 4 en 5) zijn moeilijker te vergelijken/onderscheiden dan getallen die verder uit elkaar liggen (zoals 5 en 9). Dit verschil is te wijten aan overlappende (ruimtelijke) representaties die resulteren in het zogenaamde afstandseffect (distance effect), dat gekenmerkt wordt door een minder nauwkeurige/langzamere vergelijking van getallen die dicht bij elkaar staan dan getallen die verder weg liggen. De aanwezigheid van afstandseffecten in vergelijkingstaken wordt historisch geïnterpreteerd als een teken dat de niet-symbolische, analoge (ruimtelijke) representaties van getallen gebruikt worden. Bij kinderen (in groep 1 en 2) en jongvolwassenen waren typische numerieke afstandseffecten aanwezig op reactietijd en nauwkeurigheid voor Arabische cijfers en stip patronen in zowel de subitizing (1-4) en niet-subitizing (6-9) ranges. Echter, neurale afstandseffecten op de P2p amplitude, waarvan verondersteld wordt dat ze geassocieerd zijn met een vroeg verwerkingsstadium waarin men voor het eerst toegang heeft tot numerieke-informatie, vertoonden wel verschillen in ontwikkeling. Terwijl kinderen alleen een P2p afstandseffect vertoonden voor de subitizing range in de symbolische en niet-symbolische taken, vertoonden volwassenen P2p afstandseffecten voor beide numerieke ranges in beide taken. Deze gecombineerde resultaten leidden tot de conclusie dat alleen de Arabische cijfers 1-4 betekenis krijgen door koppelingen aan corresponderende niet-symbolische representaties van grootte in de ANS, terwijl de Arabische cijfers 6-9 andere processen vereisen dan directe niet-symbolische koppeling. Wij speculeren dat de aanwezigheid van een P2p afstandseffect voor cijfers/kwantiteiten buiten het subitizing bereik bij volwassenen voortkomt uit de verdere ontwikkeling van het symbolische getallensysteem. 
De studies in Hoofdstuk 2 en $\mathbf{3}$ leveren dus bewijs ten gunste van de Exacte Algoritme Hypothese, waarbij alleen cijfersymbolen 1-4 geleerd worden door niet-symbolische symbolische koppeling.

Het tweede doel van dit proefschrift was het onderzoeken van de semantische verwerking van cultuurspecifieke (canonieke) vinger-cijfer representaties en hoe deze de verwerking van symbolische getallen (Arabische cijfers) door volwassenen zouden kunnen vergemakkelijken in de subitizing (1-4) en non-subitizing (6-9) ranges. Het cultuurtypische patroon van opgestoken vingers om een bepaalde hoeveelheid aan te geven wordt ook wel een canoniek vinger-cijferpatroon genoemd. Het gebruik van vingers om hoeveelheden aan te geven is universeel. Kinderen tonen al heel vroeg in hun ontwikkeling het vermogen om een getal met hun vingers aan te geven, soms al voordat ze de juiste getalwoorden gebruiken, wat wijst op een mogelijk belangrijke rol van vinger-getal representaties in het vroege leren van getallensymbolen. De twee ERP-studies beschreven in Hoofdstuk 4 en 5 gebruikten verschillende taakparadigma's om te onderzoeken of er nog tekenen zijn van cultuurspecifieke vinger-cijferrepresentaties die de verwerking van andere geleerde getalsymbolen (Arabische cijfers) in de hersenen van jongvolwassenen beïnvloeden (faciliteren). Dergelijk bewijs zou wijzen op het belichaamd worden van vingerrepresentaties (embodiment) en op het potentieel belang van vingers tijdens vroege getallensymboolverwerving.

Om te onderzoeken of jongvolwassenen tekenen vertonen van belichaamde canonieke vinger-cijferrepresentaties, werd in de studie beschreven in Hoofdstuk 4 gebruik gemaakt van een wiskunde verificatie paradigma. We legden jongvolwassenen eenvoudige wiskundeproblemen voor, gevolgd door een antwoord in de vorm van een canoniek of atypisch (niet-canoniek) vinger-cijferpatroon. Deelnemers verifieerden het antwoord, in de vorm van canonieke of niet-canonieke vinger-cijfer patronen, met de op-dat-moment-geactiveerde oplossing in hun geheugen. Dit deden ze door aan te geven of het vinger-cijfer patroon en de oplossing hetzelfde of een verschillend getal toonden. De reactietijd, nauwkeurigheid en pariëtale P2p amplitudes van de deelnemers werden gemeten en vergeleken tussen correct (overeenkomende numerieke betekenis) canonieke, correct niet-canonieke, incorrect canonieke, en incorrect niet-canonieke condities. Hoofdstuk 5 onderzocht dezelfde onderzoeksvraag in een andere steekproef van jongvolwassenen, nu met behulp van een gelijk/verschillend paradigma (same/different paradigm). In deze taak, in plaats van het vergelijken van vingerpatronen met mentaal gerepresenteerde somoplossing, moesten deelnemers een Arabisch cijfer vergelijken met 
een gelijktijdig weergegeven vingerpatroon, dus binnen één stimulusdisplay. De vier condities in deze taak waren: canoniek-congruent, canoniek-incongruent, niet-canoniek-congruent en niet-canoniek-incongruent. In lijn met eerdere bevindingen vonden we dat reacties op canonieke vingerpatronen sneller en nauwkeuriger waren. Volgens eerdere auteurs zijn dergelijke faciliterende effecten van canonieke vinger-cijferpatronen op getalverwerking te wijten aan hun opslag en koppelingen met andere symbolische getalrepresentaties in het langetermijngeheugen. Deze koppelingen zorgen ervoor dat een numerieke betekenis sneller opgehaald kan worden bij het zien van een canoniek vinger-cijfer patroon. We onderzochten deze beweringen met behulp van ERP-metingen in aanvulling op gedragsmetingen, met de nadruk op de P2p en P3 ERP-componenten die verschillende stadia van semantische getalverwerking vertegenwoordigen. In overeenstemming met eerdere studies die gebruik maakten van vergelijkbare taakparadigma's, indexeert de occipitale-pariëtale P2p een vroeg cross-modaal semantisch proces. De centro-pariëtale P3 vertegenwoordigt een laat semantisch proces dichter bij de responsbeslissing, een weerspiegeling van bewuste geheugenherkenning en verhoogd responsvertrouwen.

In beide experimenten resulteerden canonieke vingerpatronen in snellere en meer accurate reacties en grotere $\mathrm{P} 2 \mathrm{p}$ en $\mathrm{P} 3$ pieken in de rechterhemisfeer in vergelijking met nietcanonieke patronen. Deze neurale metingen bevestigen de hypothesen van eerdere studies; canonieke vinger-patronen lijken belichaamd te zijn geworden en semantische associaties te hebben ontwikkeld met andere getalrepresentaties, waardoor de numerieke betekenis sneller kan worden opgehaald en de verwerking van andere getallen wordt vergemakkelijkt. In overeenstemming met de studies in Hoofdstuk 2 en $\mathbf{3}$, onderzochten deze studies ook de faciliterende effecten van canonieke vinger-cijfer patronen op de verwerking van Arabische cijfers, afzonderlijk voor de subitizing (1-4) en non-subitizing (6-9) ranges. De resultaten toonden verbeterde prestaties en sterkere neurale reacties op canonieke vingercijfer patronen in het subitizing bereik in vergelijking met hogere cijfers in beide taken. Echter, de studie beschreven in Hoofdstuk 4 toonde ook facilitatie in het non-subitizing range wanneer de oplossing overeenkwam met de verwachting en in het subitizing bereik wanneer de oplossing niet overeenkwam met de verwachting. Deze verschillen tussen de studies kunnen niet verklaard worden door de huidige data en moeten verder onderzocht worden. Bovendien toonden beide studies rechts-hemisferische lateralisatie van de P2p boven occipitaal-pariëtale elektrode locaties. Gebaseerd op eerdere studies, interpreteerden wij deze lateralisatie voorlopig als een teken dat belichaamde canonieke vinger-cijfer patronen meer lijken op iconen of ideografieën; visuele representaties van een idee (zoals 
een emoji), los van taal. Deze gegevens suggereren dat canonieke vinger-getal patronen een belangrijke rol zouden kunnen spelen in vroege getalverwerving, vooral voor kleine getallen in de subitizing range.

De studies gepresenteerd in dit proefschrift geven meer inzicht in de neurale mechanismen die ten grondslag liggen aan het leren van getallensymbolen en de faciliterende functie van canonieke vinger-cijferrepresentatie. Deze bevindingen en de gebruikte methoden/paradigma's kunnen helpen bij de opzet van toekomstige studies die het vroegtijdig leren van getallensymbolen onderzoeken binnen uitgebreidere longitudinale ontwerpen. Uiteindelijk zouden de neurale correlaten van getallen/getalverwerking die in onze studies worden gerapporteerd ook nuttig kunnen zijn om de individuele verschillen in getalverwerking te onderzoeken in de vroege ontwikkeling die grote gevolgen kunnen hebben in latere jaren. Nu de samenleving steeds digitaler en afhankelijker wordt van numerieke informatie, wordt het vermogen om getalsymbolen te verwerken steeds belangrijker.

\section{Referenties}

Carey, S. (2009). The origin of concepts (Volume 133). Oxford University Press. https: //doi.org/10.1093/acprof:oso/9780195367638.001.0001. (Cited on page 239)

Carey, S., \& Barner, D. (2019). Ontogenetic origins of human integer representations. Trends in Cognitive Sciences, 23(10), 823-835. https://doi.org/10.1016/j.tics. 2019.07.004 (cited on page 239)

Dehaene, S. (2001, February). Précis of the number sense. Mind \& language (Pages 16-36). https://doi.org/10.1111/1468-0017.00154. (Cited on page 239)

Piazza, M. (2010). Neurocognitive start-up tools for symbolic number representations. Trends in Cognitive Science, 14(12), 542-551. https://doi.org/10.1016/j.tics.2010. 09.008 (cited on page 239) 


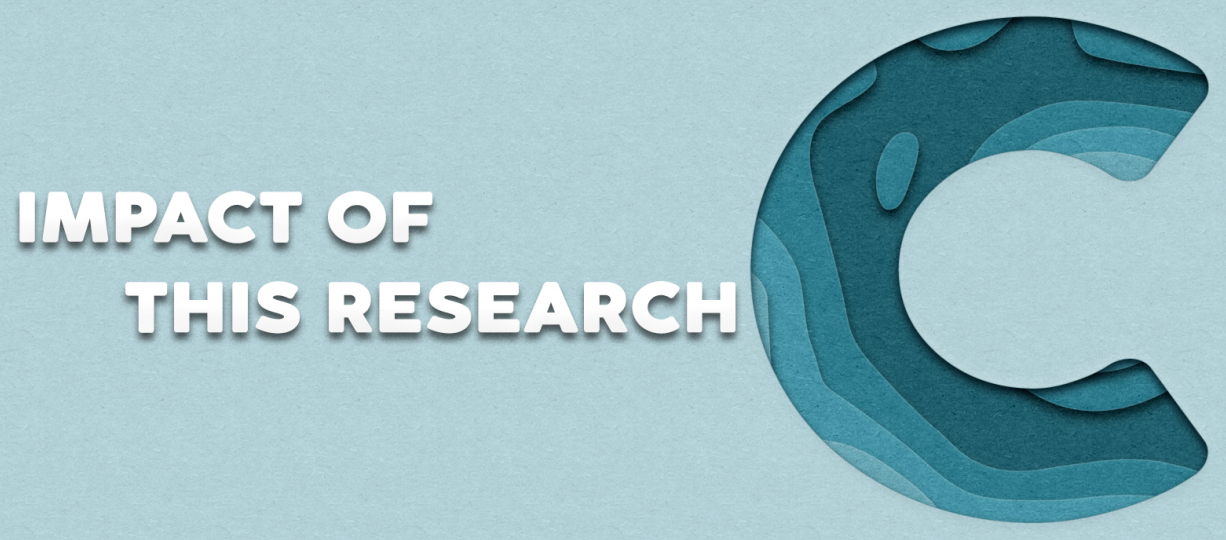

\section{Impact of this Research}

Numbers are abundant in our current society; they are everywhere, from the price tags in shops to the excel sheets we use for our jobs. These arbitrary symbols inform us about exact quantities. There is no doubt that 57 is one more than 56 , a comparison that is impossible for real-world non-symbolic representations (such as two flocks of birds). Using these numbers does not come naturally; it is a skill taught to children at an age most of us do not even remember. Learning numbers is a difficult task and takes years to master fully. This dissertation focused on the beginning of that journey: how number symbols gain their numerical meaning and how the brain represents and semantically processes symbols (e.g., Western Arabic numerals and finger gestures). We especially looked at the distinction between the smaller subitizing range (numbers 1 through 4) and the non-subitizing range (more than 4 ). 
The first two chapters in the current work investigate whether numerical symbols (such as '4' or '7') gain their meaning by mapping directly onto non-symbolic representations of these quantities (such as $\bullet \bullet \bullet)$. In both studies, we made a distinction between the subitizing range (1-4) and non-subitizing range (6-9), measuring the behavioral performance and Event-Related Potentials (ERP) of adults (Chapter 2) and children (Chapter 3). Both studies suggest that learning through direct mapping only occurs for the subitizing range. The non-subitizing range is eventually attained through other processes, likely algorithms tied to the symbol-to-symbol associations inherent to the symbolic number systems (such as counting or a later-is-greater principle). The third and fourth chapters look at the role finger-number gestures might have in number processing. These chapters show that, in adults, these culturally acquired finger patterns still facilitate the processing of Arabic numerals, but more so for the smaller subitizing range than the larger non-subitizing range. This difference between ranges suggests that the connection between finger patterns and the quantity they represent is stronger for the smaller range, possibly due to more intensive use during early development.

The results mentioned above contribute to the investigation of numerical development by demonstrating that direct mapping between a non-symbolic quantity and a numerical symbol can only occur for the subitizing range. These results strengthen the claims of more recent models on numerical development that emphasize the subitizing range. A strength of the current dissertation is also the inclusion of neural measures (in the form of Event-Related Potentials: ERPs) besides end-point measures such as response time and accuracy. ERPs provide additional information at a millisecond-level about effort invested in early and later semantic processing stages that precede decision making. The studies in the current dissertation are among the first to show the dynamic changes in neural N1 and P2p components during the symbol learning process (Chapter 2). Such changes might not be detectable with behavioral measures alone, as Chapter 3 demonstrates. The addition of neural measures could be a key factor in distinguishing between numerical and nonnumerical processes that lead to these distance effects. Chapters 4 and 5 were also among the first studies to report on the sensitivity of P2p-ERP components to finger-number gesture canonicity differences. These results broaden the $\mathrm{P} 2 \mathrm{p}$ to a marker for embodied numerical representation in addition to symbolic and non-symbolic numbers. The current results, and those in future studies that might use these measures, could help determine how the symbolic number system and wider numerical cognition develops in children. 
Numerical literacy is crucial in today's world, with nearly every activity involving numbers to some extent. A good grasp of informal skills (such as counting or estimating) before school is a big advantage for formal mathematical education in school. Gaining a better understanding of how children develop these early numerical skills becomes increasingly more important. A good understanding of mathematics has further implications throughout life, being linked to job success, social mobility, and even mental and physical health costs. While the current work doesn't directly influence these societal aspects of mathematics education, it lays the groundwork in studying the factors that contribute to early symbol learning. The investigation into finger-number symbols is a good example of such factors, showing that these can be beneficial to learning the first four symbols. This phase of acquiring the first four symbols is fundamental and, if replicated, could inform new education programs on how to improve number learning. Likewise, the findings that the non-subitizing range cannot be mapped directly onto non-symbolic quantities could lead to programs that focus more on the inter-symbol associations that contribute more to learning these numbers. However, more research is needed to see which processes are involved in learning the meaning of numerals in the non-subitizing range.

The current dissertation is only a small piece of the puzzle. The findings presented here can enrich current paradigms that mainly focus on behavioral measures with relatively easy-to-detect ERP markers that can be used at any developmental stage. These initial findings pave the road towards a more comprehensive model of numerical development that can describe which numerical abilities children have at which age. Such a model provides a scaffold for researchers and teachers to improve teaching methods and prepare children for an increasingly number-heavy world. 



\section{ACKNOWLEDEEMENUS}

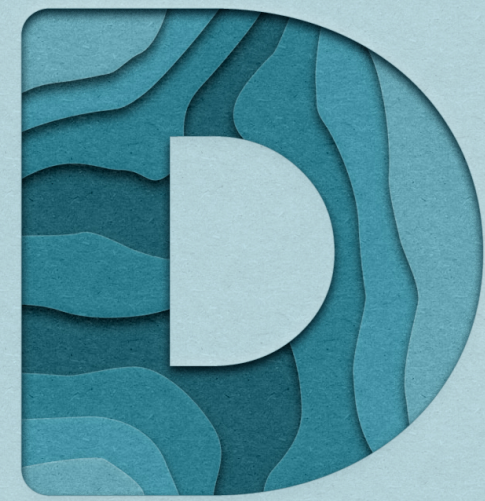

\section{Acknowledgements}

I would like to thank Lisa for supervising me and giving me the honest feedback and guidance needed to grow as a scientist, as well as Peter for his insightful comments and invaluable input to the projects. I always enjoyed meetings with the three of us, where I learned more about how the university works than anywhere else. I also appreciated the freedom both of you gave me in pursuing different paths and analyses, which led to some of the most interesting conclusions in my $\mathrm{PhD}$ and sometimes didn't lead anywhere at all. I would also like to thank the developmental group as a whole for taking me in. I still miss the great hallway conversations with Hans and Hanneke.

A lot of gratitude goes to the friends I made in the last few years. Selma, Alex, and Marta, at first it was usually the four of us occupying the second floor. Somehow this escalated to hijacking couches to create space for anywhere between fifteen and thirty people (especially if free food was involved). Strictly 12:00 was lunch time and we made sure to fetch anyone who missed the mark. Aline, your hugs and support were unmatched, where would I be without those? There's also nobody I'd rather drink 30 days of cornstarch for than you. I liked it so much I did it twice! Alix, seeing you speed through the halls gave me energy, your perseverance and strength was inspiring. I also never felt more special than when you invited me to come see your cells. Danny, you were a ghost at the department and your presence was always a whole event, pretty sure we've spent more time in the gym together than at the office. What great conversations we had when working out though. Giada! You were such a happy and positive presence! Meowing away and 
reminding us (and yourself) that "we are not our data." Hannah, always such a pleasure seeing you drag around heavy equipment back and forth from the hospital, we shared quite a few MATLAB frustrations, but that never got us down. The penguin will always be here waiting when the others kick you off the couch for scary stories. Olof, your positivity was infectious, and I'll never forget the Sinterklaas visit I got at the office when you first started. Peppe, the best Italian at the department! You have a great presence, and I will never say no to your pizza parties, I promise to bring my protein bars! Vaish, did you ever complete the puzzles I made you? I enjoyed our lunch talks about plants and annoying you with terrible Bollywood movie suggestions, we're going to watch one of those someday! Yawen and Mike, the few people beside me to still work at the office in 2020! Loved the hallway talks with you both and doing experiments with Yawen. And also thanks to the occasional visitors for lunch, Mathilde, Miriam, Alex, and Linda, for making the whole $\mathrm{PhD}$ experience a positive one.

Eliza, you were often an inspiration for what hard work can get you, to not give up, and keep fighting for what you want! I truly admire that in you, and I am proud to call you my friend. Anne, one of the people I've known the longest! We started university at the same time but took wildly different paths. But look at us now, still here and thriving! Wonder what we'll be up to in another 10 years. Miriam, we also started university together and now we are finishing a PhD together, so many years of school and we're finally done! I'm sure we can laugh about this whole thing in a couple of years over drinks and a pizza. And a thanks to Nina, Sanne, and unofficially Kirsten, with whom I shared an office and plenty of laughs.

Shanice, I had zero doubt about who I would ask to be my paranymph, of course it had to be you! We've supported each other through so many things, so having you watch my back during the defense only feels natural. I don't see this changing anytime soon, and expect many more birthdays, dinners, and walks in our future. We are a perfect mix of opposites and complements for the best kind of friendship. 
Julian, it didn't take me very long to decide who my other paranymph would be either. You've been a great support throughout the years, always there for lunch, and a wonderful boxing partner! There will be many more movie nights and gaming sessions in our future, that's for sure. I appreciate your friendship a lot and can't imagine I would've managed this $\mathrm{PhD}$ the way I did without all those positive moments.

To Marie, one of my best friends in the world, I literally could not have managed the last few years, especially the lockdown year, without your incredible support and constant positivity. You cheered me on and motivated me to get through it all. I can always rely on your honesty, and even if we disagree you are still on my side. I appreciate you more than I can say.

Lena, you've been a great friend these past few years. All the movies, walks, and talks helped me keep my sanity. You've been a permanent distraction in the best kind of way and gave me the much-needed relief from my own work every now and then.

A huge thanks to my Mom, Dad, and the rest of my family, for not just supporting me during the $\mathrm{PhD}$, but everything that came before and is going to come after. I would not be here today without the lessons taught to me by my parents and siblings, and I would not have had as many relaxing days if it wasn't for the kids. Playing games with Tycho and Lyna is always a highlight and getting pictures of Axel, Alexis, Nejc, and Laura would make my day.

It would not be a complete acknowledgement without also thanking all the friends I made that I haven't met in person yet. The wonderful community of scientists and kind individuals online that are just as important as the people you see in person. The conversations I've had with brilliant individuals across the globe made the work so much easier and less lonely. Special shout-outs go to wonderful people that embody the term amazeballs such as Jolanta, Brooke, Dagmar, Duncan, Helena, Vee, Julia, and Eva.

A PhD cannot be attained in a void without losing a part of yourself. I realize now more than ever how much small encouragements, a listening ear, or a short distraction can improve your life in a big way. I am grateful for everyone who has been there for me, who made me a part of their lives and were kind enough to be a part of mine. The past four years have changed me more than just gaining a title. Thank you all. 



\section{ABOUI UHE AUThOR 8 PUBLICAILNS}

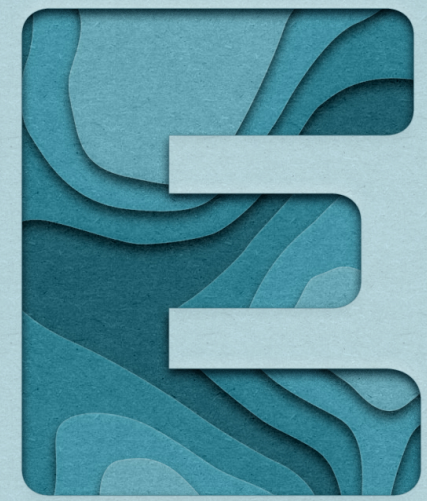

\section{About the Author}

Fabian van den Berg was born on July 16th, 1990 in Roermond, Limburg, the Netherlands. He grew up in the small town of Beesel and aspired to study Psychology. Due to how the Dutch education system was set up, he could not attend University after finishing High School, leaving him with two choices: two more years of High School at a higher level or chance gaining enough credits with one year of Higher Professional Education (Hoger Beroepsonderwijs). Naturally, he chose the latter and attended one year of Social Work, completing the propaedeutic year needed to advance to University (and learning some psychology and drama along the way).

In 2009 he started his studies at Maastricht University, with the 3-year Psychology program. During this program, his curiosity was sparked, and a love for research developed, leading to a more biologically focused set of courses in the third year. This curiosity was further encouraged during the special MARBLE excellence program, that allowed him to conduct his first study and present the findings during the international student conference. However, he never fully let go of the clinical/practical side of psychology and neuroscience. In 2012 he was admitted into the Research Masters Cognitive and Clinical Neuroscience, with a specialization in Neuropsychology. In this program, he was able to pursue his research aspirations and gain clinical experience and certifications in a private practice in Eindhoven. With a clear view of both research and the clinical world, the decision was made much easier, and he knew for certain that research was where his heart lies. 
After finishing the research master's in 2016, Fabian started applying for various $\mathrm{PhD}$ programs across the Netherlands. During the next two years, he also worked as a statistician, using the knowledge and skills gained teaching statistics during his student days. Fate would have it that he would find his way back to Maastricht, working with Lisa Jonkman on a $\mathrm{PhD}$ project investigating the neurocognitive processes involved in numerical processing. During this four-year project, he would also teach various courses in Developmental Psychology, Statistics, Excel, and even create and teach a series of classes on advanced statistical methods within the PhD graduate school. Upon completing his $\mathrm{PhD}$, he will continue to explore his curiosity for data, human behavior, and helping others learn and improve.

\section{List of Publications}

van den Berg, F. C. G. \& Jonkman, L. M. (in prep). Longitudinal electrophysiological data provide more evidence for shared number symbol-quantity representations in the subitizing than the non- subitizing range in 5-6-year-old children.

van den Berg, F. C. G., de Weerd, P., \& Jonkman, L. M. (2020). Number-related brain potentials are differentially affected by mapping novel symbols on small versus large quantities in a number learning task. Journal of cognitive neuroscience, 32(7), 1263-1275.

van den Berg, F. C. G., De Weerd, P., \& Jonkman, L. M. (2021, under review). Canonical finger-number postures facilitate the processing of Arabic digits in adults: an Event-Related Potential study. Neuropsychologia

van den Berg, F.C.G., de Weerd, P., \& Jonkman, L. M. (2021). Electrophysiological Evidence for Internalized Representations of Canonical Finger-Number Gestures and their Facilitating Effects on Adults' Math Verification Performance. Nature Scientific Reports, 11(1), 11776. https://doi.org/10.1038/s41598- 021-91303-2

\section{Conference Presentations}

EARLI SIG 22 - Neuroscience and Education Conference; London 2018. Learning Numbers: Effects of Learning new Number Symbols on Number Processing-Related Brain Activity 


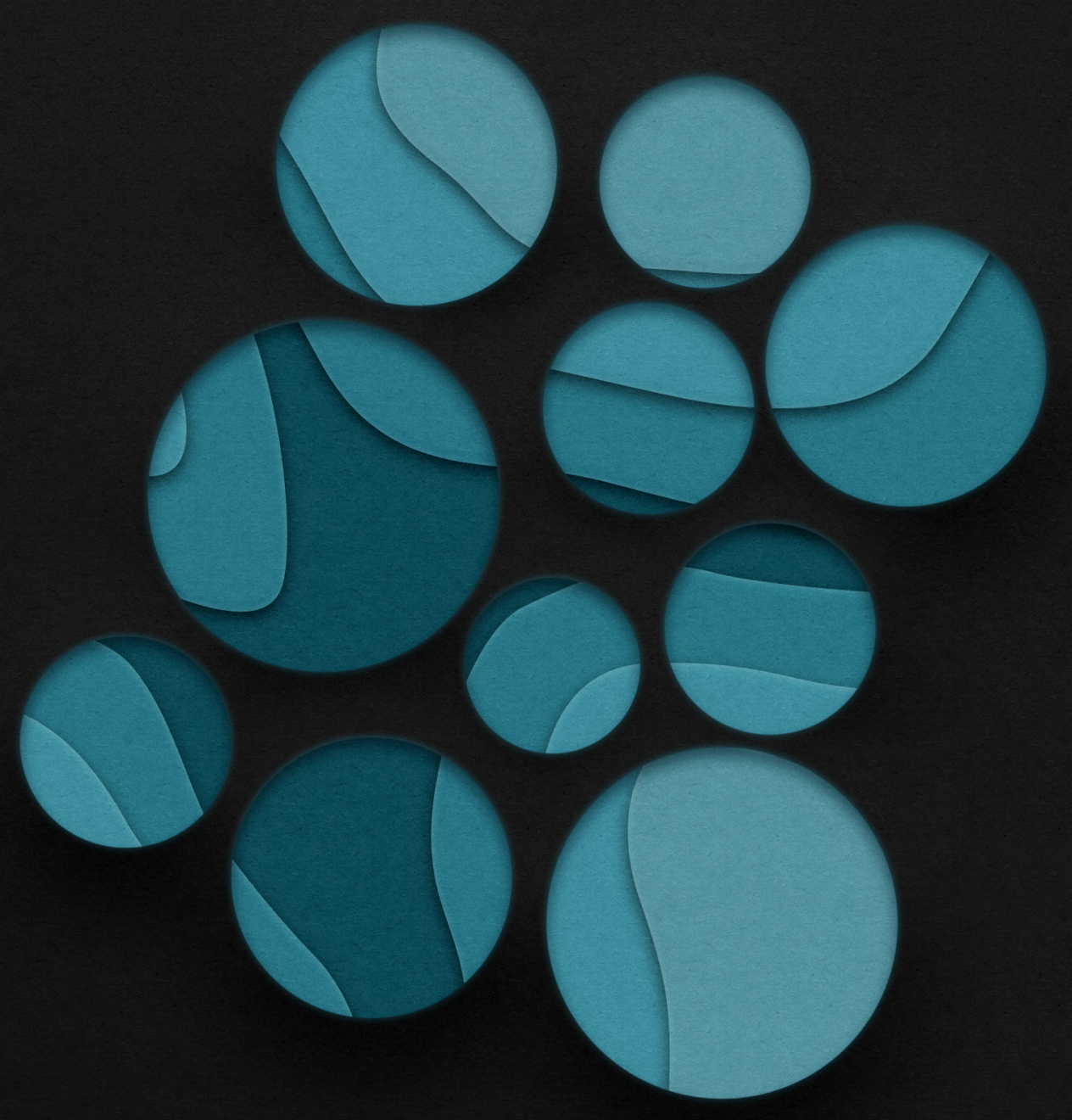

$$
\begin{aligned}
& 45 \sqrt{3} \\
& \text { अ) \&्वनक } \\
& \text { 染別现 }
\end{aligned}
$$

